\title{
Unfallgeschehen mit Lkw-Beteiligung unter Berücksichtigung von Leicht-Lkw-Kombinationen
}

Michael Hamacher

Jan Ludwig

Axel Malczyk 


\section{Unfallgeschehen mit Lkw-Beteiligung unter Berücksichtigung von Leicht-Lkw-Kombinationen}

Dr.-Ing. Michael Hamacher

Dipl.-Ing. Jan Ludwig

Dr.-Ing. Axel Malczyk

Unfallforschung

der Versicherer 


\section{Impressum}

Gesamtverband der Deutschen Versicherungswirtschaft e. V.

Unfallforschung der Versicherer

Wilhelmstraße 43/43G, 10117 Berlin

Postfach 0802 64, 10002 Berlin

E-Mail: unfallforschung@gdv.de

Internet: www.udv.de

Facebook: www.facebook.com/unfallforschung

Twitter: @unfallforschung

YouTube: www.youtube.com/unfallforschung

ISBN-Nr.: 978-3-939163-73-2

Redaktion: Dr.-Ing. Axel Malczyk

Bildnachweis: UDV und siehe Quellenangaben

Erschienen: 12/2016 


\section{Unfallgeschehen mit Lkw-Beteiligung unter Berücksichtigung von Leicht-Lkw-Kombinationen}

Bearbeitet durch:

Forschungsgesellschaft Kraftfahrwesen mbH Aachen

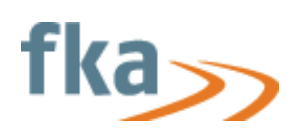

Bei der UDV betreut von:

Dr.-Ing. Axel Malczyk

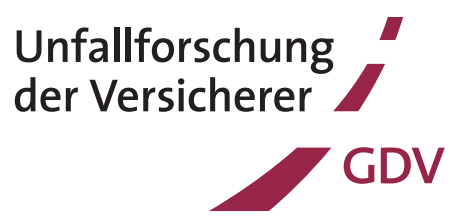





\section{Inhalt}

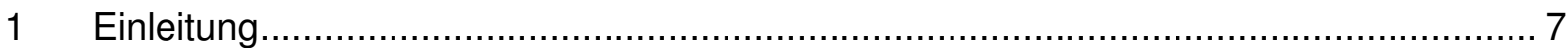

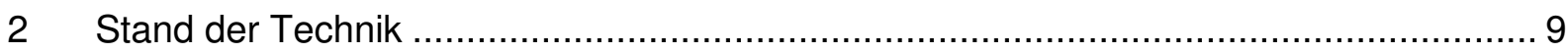

2.1 Sicherheitsanforderungen an Güterkraftfahrzeuge und Schutzmaßnahmen ........... 9

2.1.1 Crashsicherheit des Fahrerhauses.............................................................. 10

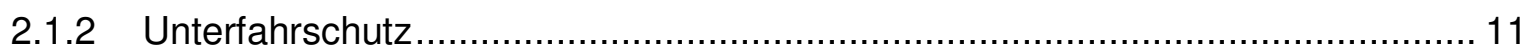

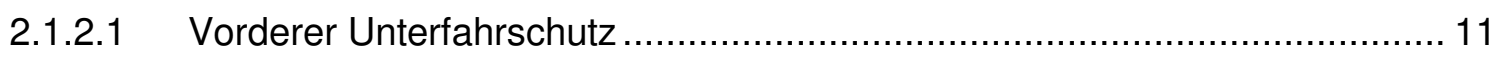

2.1.2.2 Seitlicher Unterfahrschutz ................................................................. 12

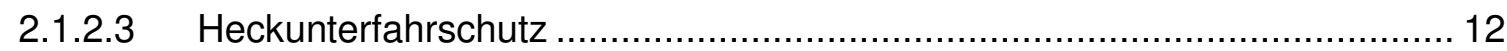

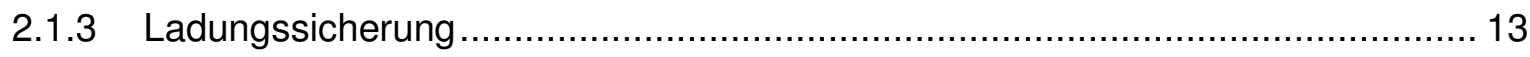

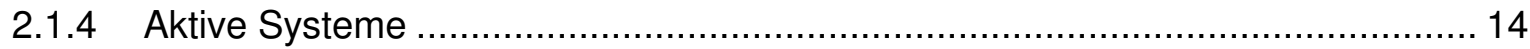

2.1.5 Manipulationen an Einrichtungen zur Lenkzeitdokumentation ........................... 16

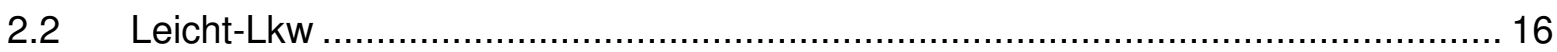

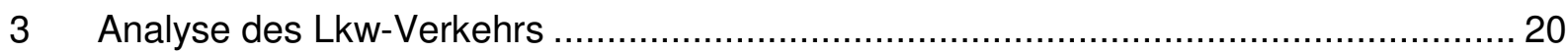

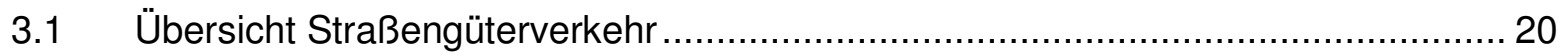

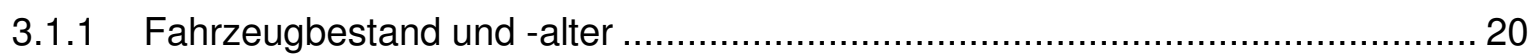

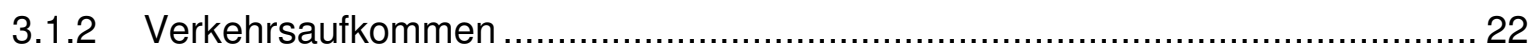

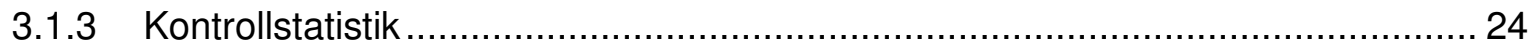

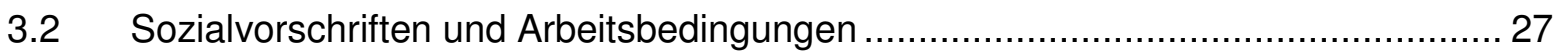

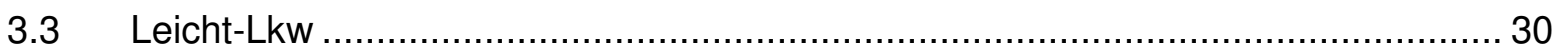

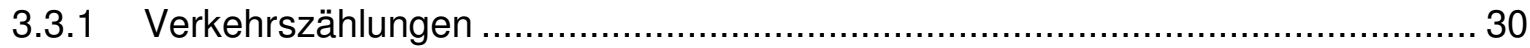

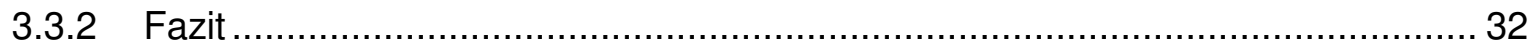

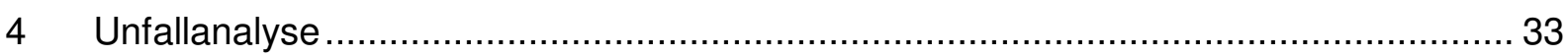

4.1 Unfälle von Güterkraftfahrzeugen über 3,5 t zGG im Straßenverkehr .................. 33

4.1.1 Bundesstatistik im Vergleich zur Unfalldatenbank der Versicherer .................... 33

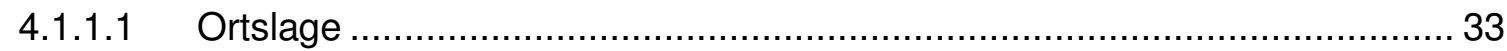

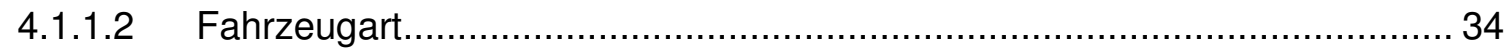

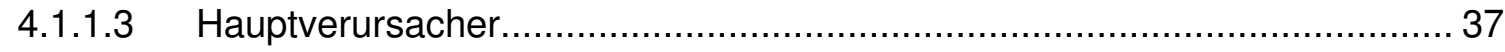

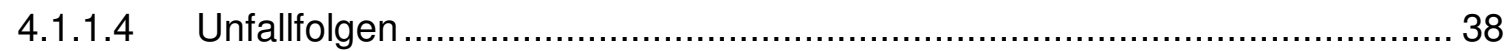




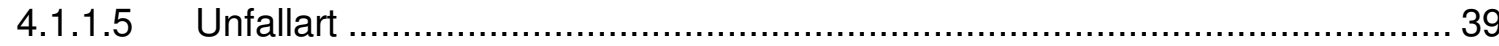

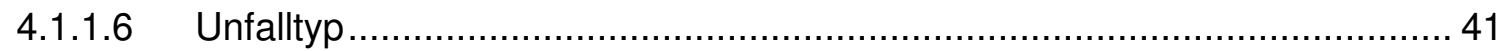

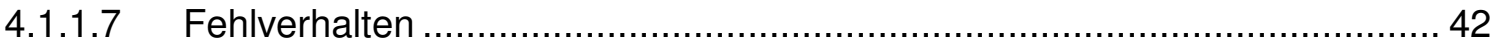

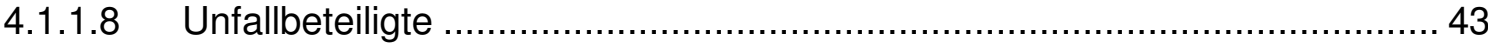

4.1.2 Weitere Auswertungen der Unfalldatenbank der Versicherer ........................... 45

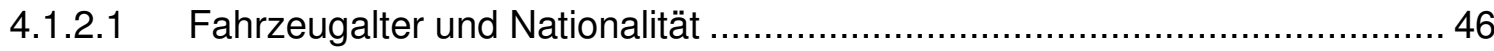

4.1.2.2 Fahreralter und Dauer des Führerscheinbesitzes.................................. 47

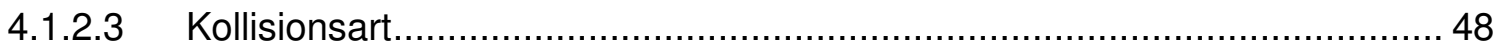

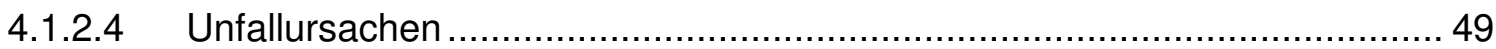

4.1.2.5 Fahrgeschwindigkeit und Bremsverhalten ......................................... 51

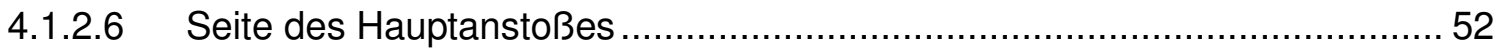

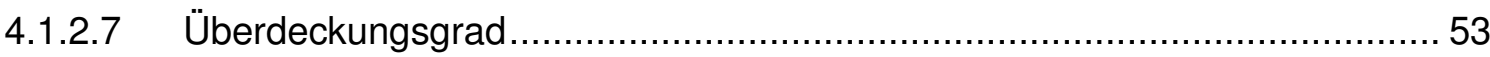

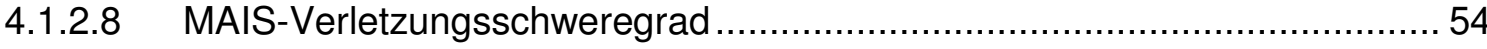

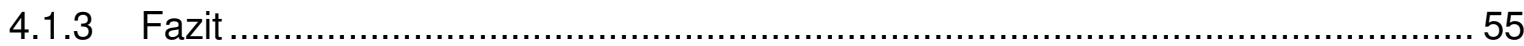

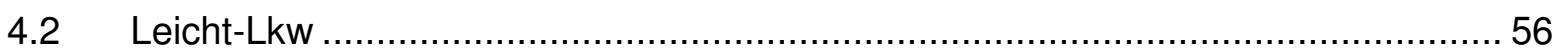

4.2.1 Sonderabfrage in der amtlichen Statistik ................................................... 56

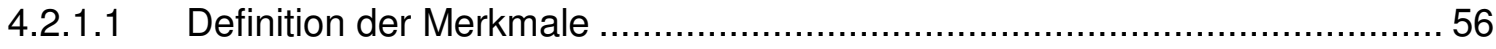

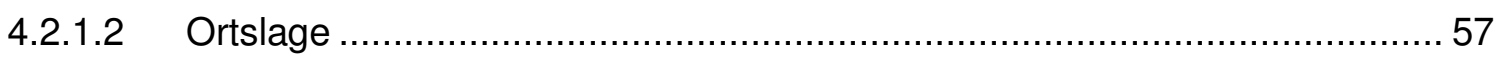

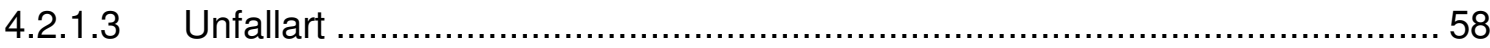

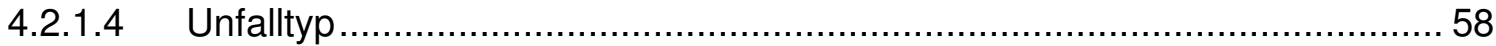

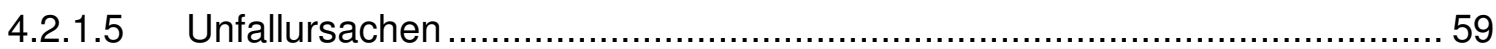

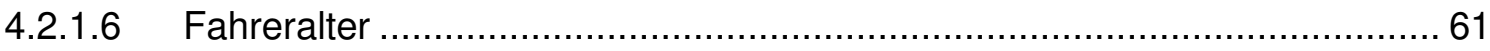

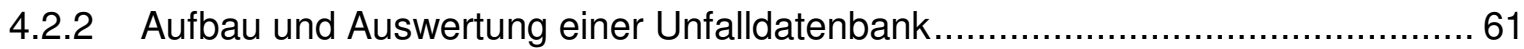

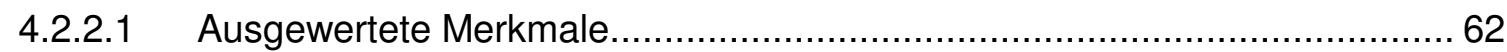

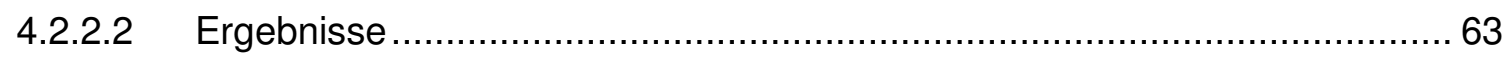

4.2.3 Auswertung von Schadenakten der Versicherer .............................................66

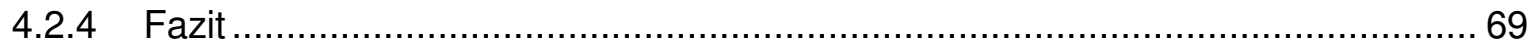

$5 \quad$ Betrachtung der Kippstabilität bei Seitenwind mittels rechnerischer Simulation ........... 71

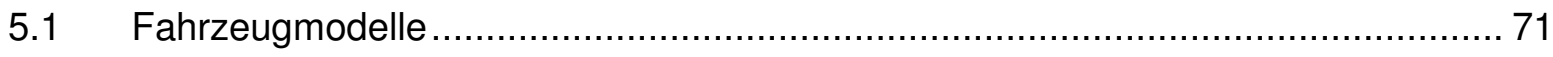

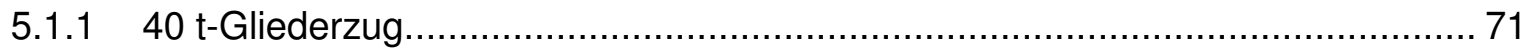

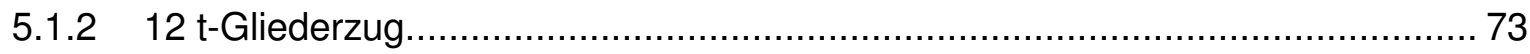




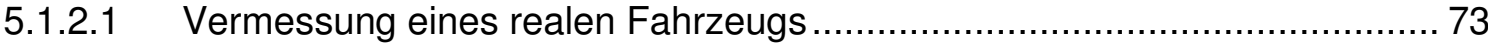

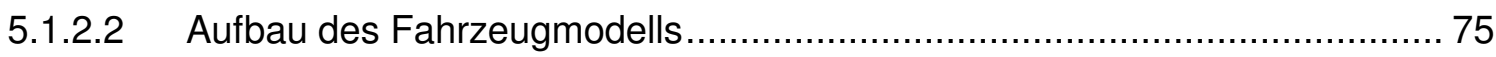

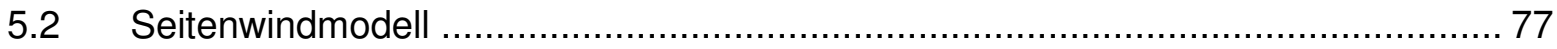

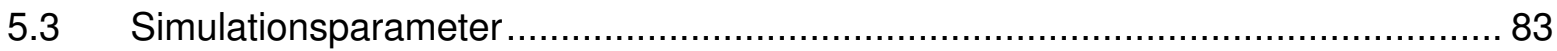

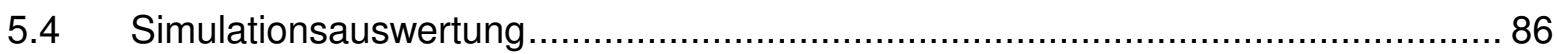

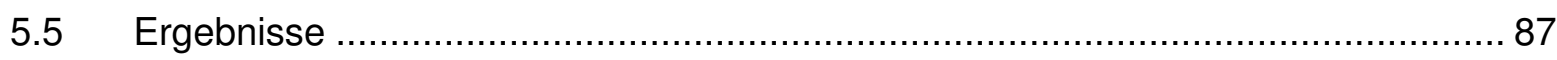

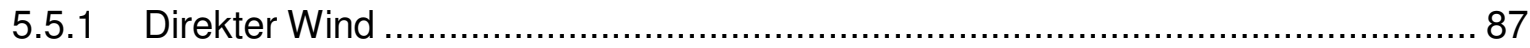

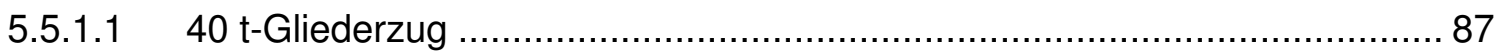

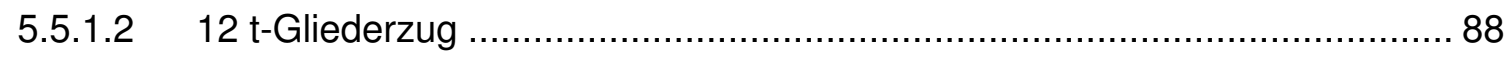

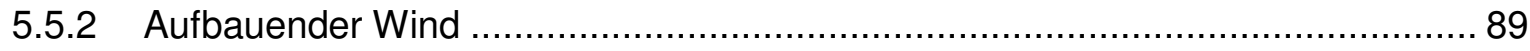

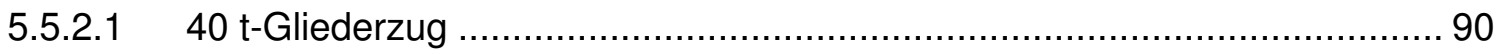

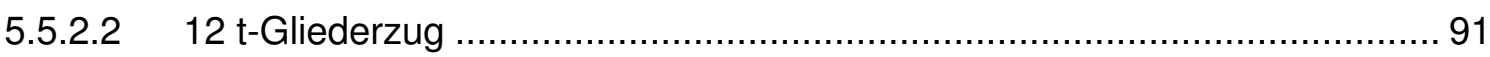

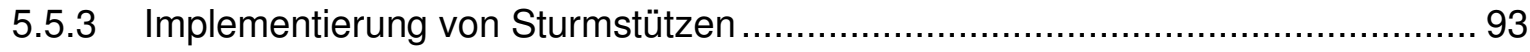

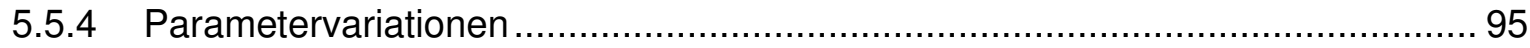

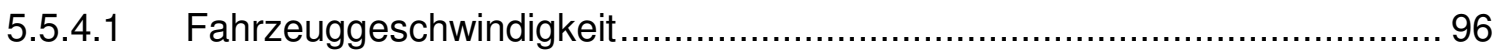

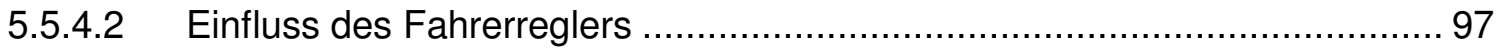

5.5.4.3 Ungleicher Beladungszustand von Zugfahrzeug und Anhänger .................. 98

5.5.4.4 Implementierung einer zweiten Anhängerachse beim $12 \mathrm{t}$-Gliederzug ........ 98

5.5.4.5 Auflastung des Anhängers beim $12 \mathrm{t}$-Gliederzug ................................... 99

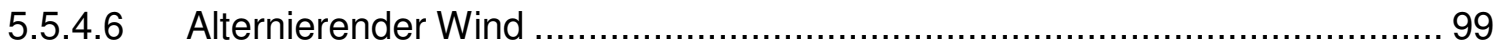

5.5.4.7 Einfluss überkritischer Windgeschwindigkeiten .................................. 100

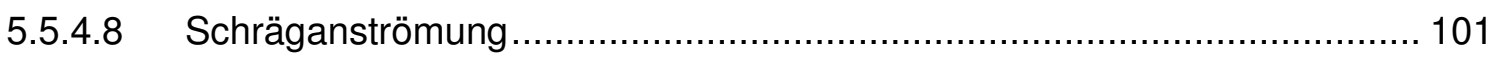

5.5.4.9 Anhänger mit geöffneter Plane .......................................................... 102

5.5.4.10 Einsatz von Ballasttanks bei Leerfahrten.............................................. 103

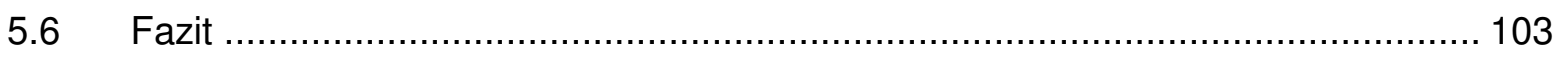

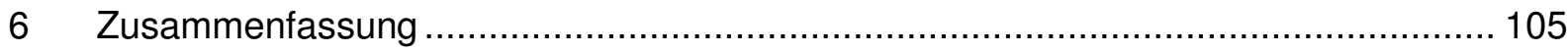

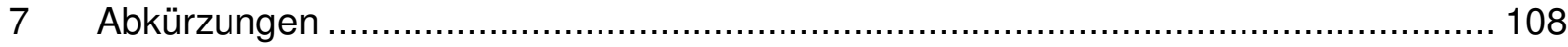

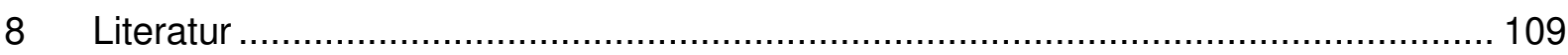

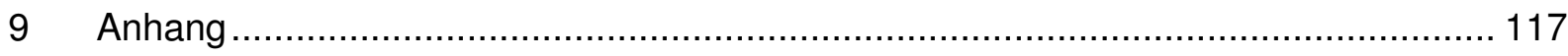

9.1 Bundesstatistik im Vergleich zur Unfalldatenbank der Versicherer.................... 117 


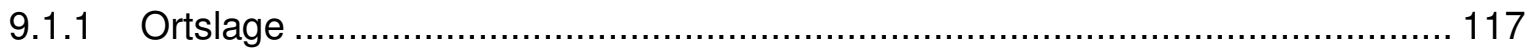

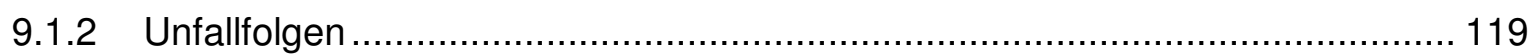

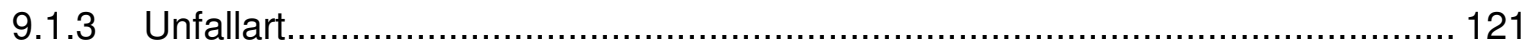

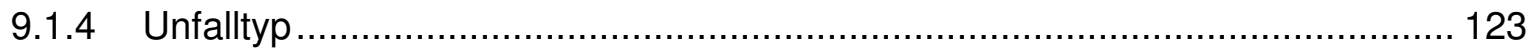

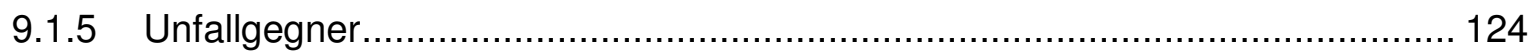

9.2 Weitere Auswertungen der Unfalldatenbank der Versicherer ............................. 126

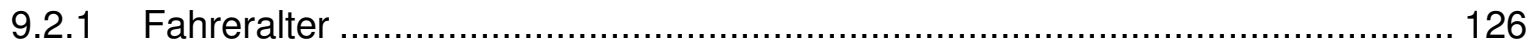

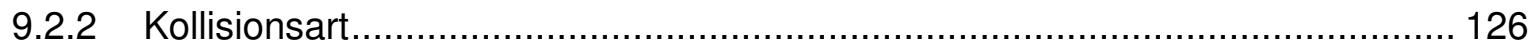

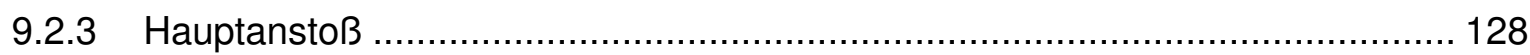

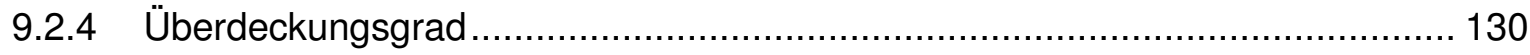

9.3 Seitenwindmodell - Mechanik des Kippvorgangs ............................................. 132

9.4 Betrachtung der Kippstabilität bei Seitenwind mittels rechnerischer Simulation ... 133 


\section{$1 \quad$ Einleitung}

Im Rahmen dieses Projektes werden das Unfallgeschehen sowie Aspekte der Verkehrssicherheit von Lkw mit einem zulässigen Gesamtgewicht über 3,5 t in Deutschland näher untersucht. Bei Betrachtungen zum Unfallgeschehen mit Lkw stehen häufig vor allem Güterkraftfahrzeuge ab $12 \mathrm{t}$ zulässigem Gesamtgewicht (zGG) im Fokus, da hier aufgrund der hohen Massen die Unfallfolgen für alle Beteiligten besonders schwerwiegend sind. Allerdings liegt in diesem Projekt ein besonderer Schwerpunkt auf Gliederzügen mit extrem leichter Bauweise, welche in den letzten Jahren zunehmend Verbreitung gefunden haben, da hiermit bis Oktober 2015 die 2005 für Lkw ab 12 t eingeführte Mautpflicht auf deutschen Fernstraßen umgangen werden konnte. Problematisch ist in erster Linie die hohe Windanfälligkeit dieser Leicht-Lkw, welche vollbeladen und bei gleichen Abmessungen immer noch etwa drei Tonnen weniger wiegen als übliche $40 \mathrm{t}$-Fernlastzüge im leeren Zustand. Die Kombination aus geringem Gewicht und einer großen seitlichen Windangriffsfläche wird als ursächlich für zahlreiche Alleinunfälle solcher Fahrzeuge angesehen und soll in diesem Projekt umfassend analysiert werden.

Die verschiedenen Arten von Güterkraftfahrzeugen und Gespannen bzw. Kombinationen werden in den Auswertungen zum Unfallgeschehen mittels Fahrzeugklassen charakterisiert. Während der klassische Pkw gemäß EG-Richtlinie 2007/46/EG in die Fahrzeugklasse M bzw. M1 fällt, gehören Güterkraftfahrzeuge der Klasse $N$ an. Unterschieden wird hier zwischen den Klassen N1, N2 und N3, die über das zulässige Gesamtgewicht des Fahrzeugs definiert sind. So weisen Fahrzeuge der Klasse N1 ein zGG bis 3,5 t auf, während das zGG von N2-Fahrzeugen von über 3,5 t bis zu $12 \mathrm{t}$ reicht. Entsprechend verfügen N3-Fahrzeuge über ein zGG von mehr als $12 \mathrm{t}$. Auch Anhänger werden in einzelne Klassen gegliedert. Im Rahmen dieses Forschungsprojekts sind in erster Linie die Anhängerklassen $\mathrm{O} 3$ und $\mathrm{O} 4$ relevant, d.h. Anhänger mit einem zGG von über $3,5 \mathrm{t}$ bis zu $10 \mathrm{t}(\mathrm{O} 3)$ sowie Anhänger mit einem $z G G$ von mehr als $10 \mathrm{t}(\mathrm{O} 4)$ [HOE13].

Im Zusammenhang mit der Verkehrssicherheit von Straßenfahrzeugen werden allgemein Maßnahmen unterschieden, die der Vermeidung von Unfällen (aktive Sicherheit) und der Verminderung von Unfallfolgen (passive Sicherheit) dienen. Die aktive Sicherheit umfasst die Bereiche Fahrsicherheit, Wahrnehmungssicherheit, Bedienungssicherheit und Konditionssicherheit. Bei der passiven Sicherheit erfolgt eine Unterscheidung zwischen Maßnahmen, die der Sicherheit der Fahrzeuginsassen (innere Sicherheit) und der Sicherheit der anderen Verkehrsteilnehmer (äußere Sicherheit) dienen [HOE13]. In Kapitel 2 werden die für Güterkraftfahrzeuge derzeit wichtigsten Anforderungen und Schutzmaßnahmen auf dem Gebiet der passiven und aktiven Sicherheit vorgestellt. Im Rahmen dieses Überblicks über den Stand der Technik werden auch die verschiedenen Fahrzeugtypen und Gespanne charakterisiert. Ein besonderer Fokus liegt dabei auf 12 t-Leicht-Lkw-Kombinationen.

Ausgangspunkt für die weiteren Arbeiten bildet in Kapitel 3 zunächst eine Analyse des Verkehrsaufkommens von Lkw über 3,5 t zGG in Deutschland. Dabei erfolgt eine Differenzierung nach Gewichtsklassen und sofern möglich auch nach Fahrzeugtyp. Untersucht werden 
Aspekte wie Bestand, Fahrleistung, Verkehrsräume und durchschnittliche Nutzungsdauer. Neben diesen rein verkehrsspezifischen Gesichtspunkten werden auch Erkenntnisse aus der Kontrollstatistik in Hinblick auf Sozialvorschriften, Ladungssicherung sowie technische Rahmenbedingungen berücksichtigt. Die Themen Sozialvorschriften und Arbeitsbedingungen werden in diesem Zusammenhang nochmals näher betrachtet. Abschließend wird speziell das Verkehrsaufkommen von Leicht-Lkw-Kombinationen auf Basis von Verkehrszählungen näher analysiert. Sowohl in Kapitel 3 als auch in Kapitel 2 fließen Ergebnisse eines Experten-Workshops ein, in dessen Rahmen die vorgenannten Themen diskutiert worden sind.

Einen zentralen Bestandteil des Projekts bildet die in Kapitel 4 vorgestellte Analyse des Unfallgeschehens von Lkw mit einem zulässigen Gesamtgewicht über 3,5 t in Deutschland. Die Ergebnisse basieren sowohl auf Daten des Statistischen Bundesamtes (Destatis) als auch auf Auswertungen der Unfalldatenbank der Versicherer (UDB). Untersuchte Merkmale sind Ortslagen, Art der beteiligten Lkw und Unfallgegner sowie die jeweiligen Unfallarten, -typen und -folgen. Ferner werden auch Fehlverhalten und der Anteil Hauptverursacher für die beteiligten Lkw-Fahrer betrachtet. Neben Vergleichen zur Bundesstatistik werden mittels der UDB-Daten noch weiterführende Auswertungen durchgeführt, die mit öffentlich verfügbaren Destatis-Daten nicht möglich sind. Ein weiterer Fokus der Unfallanalyse liegt wiederum auf $12 \mathrm{t}$-Leicht-Lkw-Kombinationen. Um deren Unfallgeschehen näher charakterisieren zu können, werden im Zuge einer Sonderabfrage beim Statistischen Bundesamt fahrzeugspezifische Auswertungen der Bundesstatistik ermöglicht. Des Weiteren erfolgen der Aufbau und die Auswertung einer Unfalldatenbank mit auf Seitenwind zurückzuführenden Leicht-Lkw-Unfällen sowie eine Analyse von Schadenakten aus der Versicherungswirtschaft.

In Kapitel 5 wird schließlich die Kippstabilität von 40 t- und 12 t-Gliederzügen unter Seitenwind mit Hilfe rechnerischer Simulation betrachtet. Dazu werden entsprechende Vollfahrzeugmodelle numerisch aufgebaut und ein Seitenwindmodell in die Simulationsumgebung implementiert. Zur Absicherung der Simulationsergebnisse und zur Untersuchung weiterer Aspekte im Zusammenhang mit Leicht-Lkw-Kombinationen werden verschiedene Parametervariationen sowie Modellanpassungen im Rahmen der Simulationen berücksichtigt. Dies umfasst auch mögliche Maßnahmen zur Reduzierung der Kippgefahr. Zusätzlich werden fahrzeugmodellseitige Einflüsse, wie die Fahrzeuggeschwindigkeit oder ein ungleicher Beladungszustand von Zugfahrzeug und Anhänger, untersucht. Zudem erfolgen Simulationen in Bezug auf das Seitenwindmodell, beispielsweise hinsichtlich der Frequenz der Windlast oder der Fahrdynamik bei überkritischen Windgeschwindigkeiten. Die Ausführungen in Kapitel 5 umfassen auch die Erkenntnisse eines zum Projektabschluss durchgeführten zweiten Experten-Workshops. 


\section{Stand der Technik}

Im Zuge der gesetzlichen Rahmenbedingungen und einsatzspezifischen Anforderungen haben sich bei Nutzfahrzeugen Klassen mit merkmalprägenden Eigenschaften herausgebildet. Strukturierungsmerkmale für 2-achsige Solo-Fahrzeuge sind die Gewichtsklassen 2,8 t, 3,5 t, 7,5 t, $12 \mathrm{t}$ und $18 \mathrm{t}$, während für 3-achsige Fahrzeuge $26 \mathrm{t}$ und für 4-achsige Fahrzeuge $32 \mathrm{t}$ zGG charakteristisch sind. Für Fahrzeugkombinationen ergeben sich je nach Anzahl der Achsen und dem dadurch begrenzten Gesamtgewicht (§ 34 StVZO) 28 t, $36 \mathrm{t}$ und $40 \mathrm{t}$ (38 t für 4-achsige Sattelkraftfahrzeuge). Daneben bestimmen die Anzahl von angetriebenen wie auch gelenkten Rädern, die Art der Federung und die dem Fahrzeuggesamtgewicht zugeordnete Motorleistung das Fahrzeugkonzept. Meist werden Gruppen von Fahrzeugen bestimmter Gewichtsklassen oder für einen gemeinsamen Verwendungszweck als Baureihe mit Abstufungen von Gesamtgewichten, Radständen und Antriebsleistungen entwickelt. Sie erhalten zur Typisierung ein einheitliches Erscheinungsbild und einen eigenständigen Namen [HOE13].

\subsection{Sicherheitsanforderungen an Güterkraftfahrzeuge und Schutzmaßnahmen}

Hinsichtlich der Fahrzeugsicherheit bestehen zahlreiche gesetzliche aber auch herstellerseitig definierte Anforderungen an Güterkraftfahrzeuge. Einen Überblick über die im Rahmen dieses Projektes relevanten gesetzlichen Anforderungen gibt Abb. 2-1.

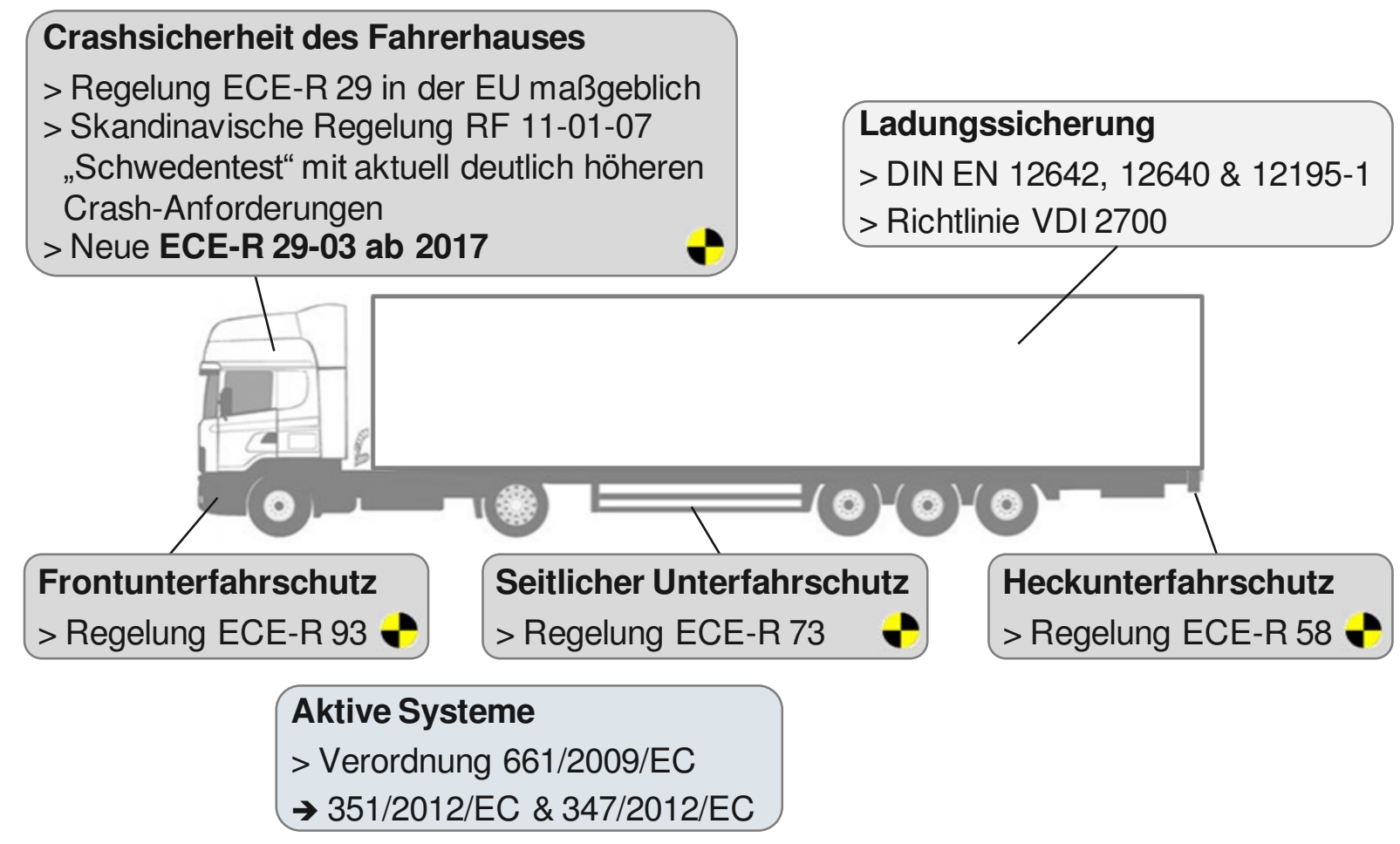

Abb. 2-1: Gesetzliche Sicherheitsanforderungen für Güterkraftfahrzeuge

Schwerpunkte auf Seiten der passiven Sicherheit bilden die Crashsicherheit des Fahrerhauses sowie die Implementierung eines vorderen, seitlichen und hinteren Unterfahrschutzes. 
Von entscheidender Bedeutung für Güterkraftfahrzeuge sind zudem die Ladungssicherung und die damit verbundenen Vorgaben. Gleichzeitig spiegelt sich die zunehmende Bedeutung von Systemen der aktiven Sicherheit ebenfalls in der Gesetzgebung wider.

\subsubsection{Crashsicherheit des Fahrerhauses}

Ab dem 30. Januar 2017 sind die erhöhten Anforderungen der Änderungsserie 03 der Regelung ECE-R 29-03 verbindlich. Diese sehen für Fahrzeuge der Klasse N mit separatem Fahrerhaus verschiedene Belastungstests vor (Abb. 2-2). Im Vergleich zur Änderungsserie 02 fällt der statische Rückwandtest weg, dafür kommen zwei Pendelschlagtests hinzu. Einmal wird mit einer rechteckigen Platte die seitliche Fahrerhausoberseite geprüft und zum anderen ist mit einem Tonnenpendel eine Frontsäulenaufprallprüfung durchzuführen, welche mit der entsprechenden Prüfung beim „Schwedentest“ vergleichbar ist, jedoch geringere Belastungen vorsieht. Bei Fahrzeugen mit einem zGG von höchstens 7,5 t entfallen die beiden neuen Pendelschlagtests.

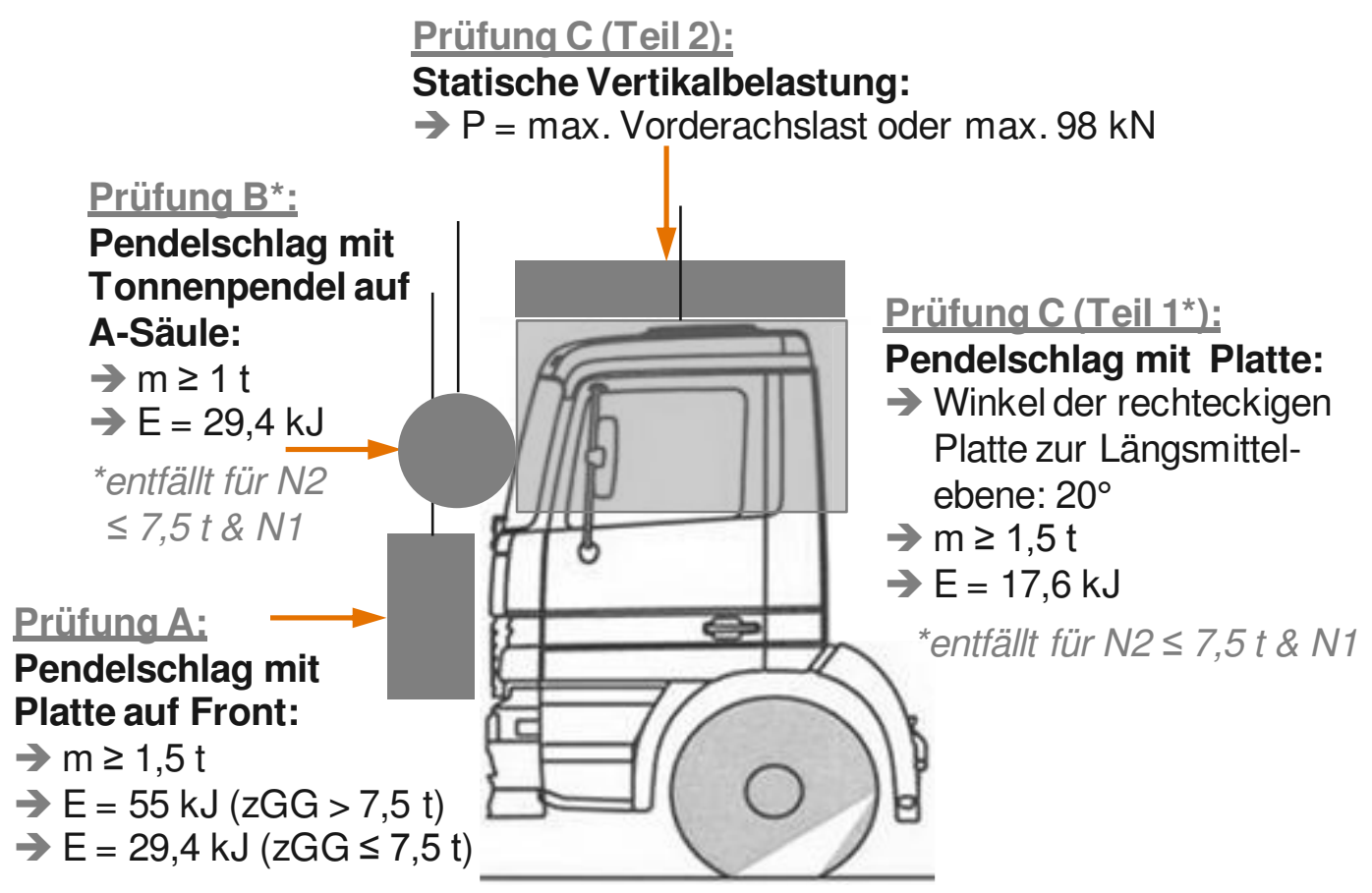

Abb. 2-2: Anforderungen an das Fahrerhaus gemäß ECE-R 29-03 (EU) [ECE10]

Für die einzelnen Prüfungen darf jeweils eine neue Kabine verwendet werden, wobei die beiden Teile der Prüfung $\mathrm{C}$ mit dem gleichen Fahrerhaus durchzuführen sind. Auf reale Tests kann verzichtet werden, sofern Simulationen oder Festigkeitsberechnungen die erforderliche Stabilität nachweisen. Die Türen müssen während der Tests geschlossen bleiben. Es ist allerdings nicht erforderlich, dass sich diese im Anschluss noch öffnen lassen.

Neben den Crashanforderungen gemäß ECE-R 29 schreibt die Regelung ECE-R 61 Mindestradien und Maximalmaße für vorstehende Außenkanten am Fahrerhaus vor, wodurch aggressive Formen vermieden werden sollen, insbesondere zum Schutz von Fußgängern und Radfahrern. 


\subsubsection{Unterfahrschutz}

Ein Unterfahren des Güterkraftfahrzeuges bzw. dessen Anhängers hat sowohl für Kraftfahrzeuge als auch für ungeschützte Verkehrsteilnehmer oftmals gravierende Folgen, sodass entsprechenden Schutzmaßnahmen eine hohe Bedeutung zukommt. Dabei unterscheiden sich die Vorgaben für den Front-, Seiten- und Heckbereich deutlich.

\subsubsection{Vorderer Unterfahrschutz}

Der Unterfahrschutz im Frontbereich ist in erster Linie auf die Kollision mit einem M1- oder N1-Fahrzeug ausgelegt und soll durch Vermeidung eines Unterfahrens der Lkw-Struktur dafür sorgen, dass die für die Energieabsorption ausgelegten Strukturen des Pkws möglichst effektiv zum Tragen kommen. Maßgeblich für die Auslegung des vorderen Unterfahrschutzes ist die Regelung ECE-R 93. Diese gilt für N2- und N3-Fahrzeuge, wobei jeweils Geländefahrzeuge (N2G \& N3G) ausgenommen sind. Die Profilhöhe des Querträgers des vorderen Unterfahrschutzes darf bei Fahrzeugen der Klasse N2 nicht kleiner als $100 \mathrm{~mm}$ und bei Fahrzeugen der Klasse N3 nicht kleiner als $120 \mathrm{~mm}$ sein. Die seitlichen Enden des Querträgers dürfen nicht nach vorn gebogen sein oder eine scharfe Außenkante haben. Diese Bedingung ist erfüllt, wenn die seitlichen Enden des Querträgers an der Außenseite mit einem Krümmungsradius von mindestens $2,5 \mathrm{~mm}$ abgerundet sind. Die horizontal aufgebrachten Prüfkräfte betragen an Prüfpunkt $P_{1}$ (Abb. 2-3, unten links) 50\% des Höchstgewichts (maximal $80 \mathrm{kN}$ ) sowie an Prüfpunkt $P_{2} 100 \%$ des Höchstgewichts (maximal $160 \mathrm{kN}$ ). Als Höchstgewicht gilt die vertikale Gewichtskraft des bis zur technisch zulässigen Höchstmasse laut Hersteller beladenen Fahrzeugs in Newton. Die Belastungsdauer beträgt mindestens $0,2 \mathrm{~s}$ [ECE10a].

\section{Statische Prüfbedingungen} gemäß ECE-R 93
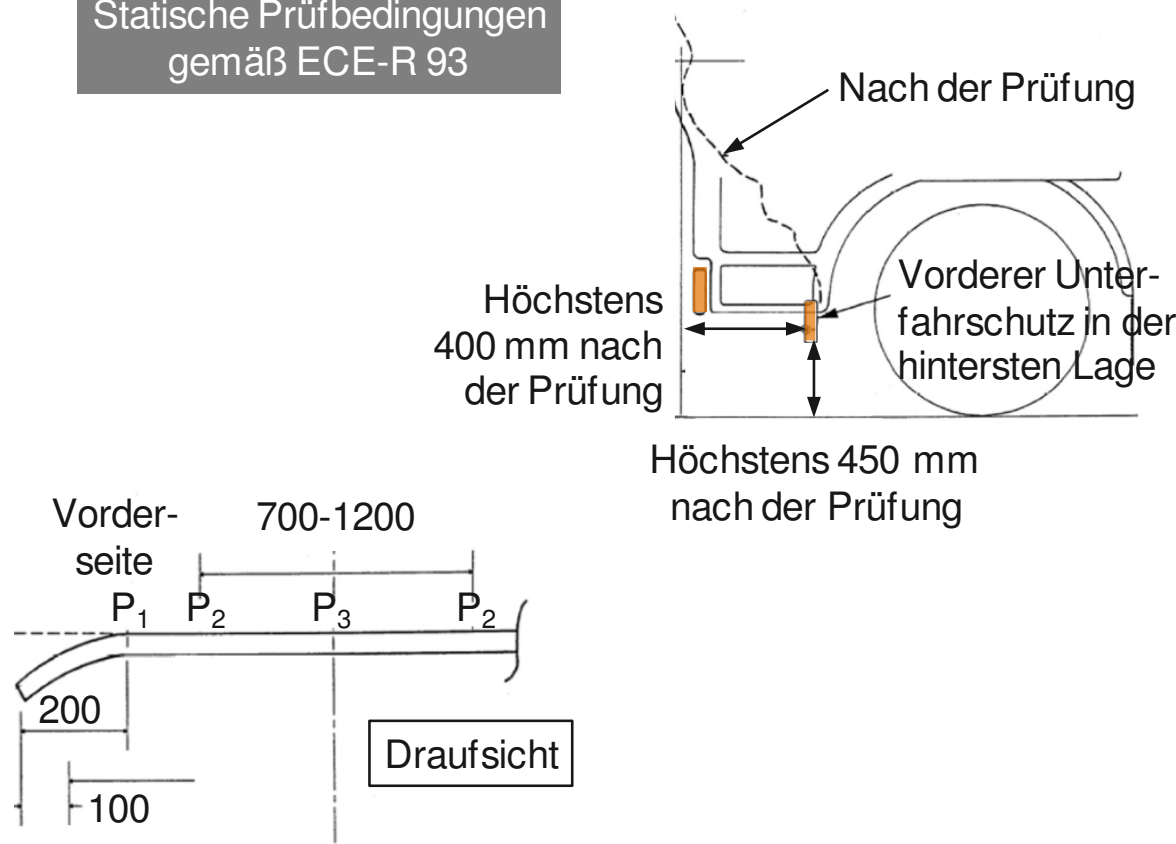

Höchstens $450 \mathrm{~mm}$ nach der Prüfung

Abb. 2-3: Anforderungen an den vorderen Unterfahrschutz [ECE10a] 
Neben der Lage der Prüfpunkte gehen aus Abb. 2-3 auch die erlaubten Verschiebungen hervor. Der vordere Unterfahrschutz muss so widerstandsfähig sein, dass nach dem Aufbringen der Prüfkräfte der horizontale Abstand zwischen dem vordersten Teil des Fahrzeugs (gemessen bis zu einer Höhe von $2 \mathrm{~m}$ ) und der Berührungsfläche des Prüfstempels am Fahrzeug nicht größer als $400 \mathrm{~mm}$ ist. Der Abstand der Unterkante des vorderen Unterfahrschutzes vom Boden darf zwischen den beiden Punkten $P_{1}$ nicht größer als $450 \mathrm{~mm}$ sein.

\subsubsection{Seitlicher Unterfahrschutz}

Der seitliche Unterfahrschutz ist nicht hinsichtlich des Anpralls eines Pkw ausgelegt. Er soll vielmehr verhindern, dass ungeschützte Verkehrsteilnehmer, also Fußgänger, Rad- oder Motorradfahrer, seitlich unter das Güterkraftfahrzeug geraten und dann von den Rädern überrollt werden. Es besteht eine Ausrüstungspflicht für alle Nutzfahrzeuge der Klassen N2 und N3 sowie alle Anhänger der Klassen $\mathrm{O} 2$ und $\mathrm{O} 4$, sofern die seitliche Aufbauunterkante in unbeladenem Zustand eine lichte Höhe von mehr als $550 \mathrm{~mm}$ über der Fahrbahn hat (§ 32c StVZO und Richtlinie 89/297/EWG). Sattelzugmaschinen, Anhänger für nicht teilbare lange Ladung (z.B. Langholz oder Stahlträger) sowie Fahrzeuge, deren praktischer Betrieb mit einer seitlichen Schutzvorrichtung unvereinbar ist (z.B. Dreiseitenkipper), sind von der Ausrüstungspflicht ausgenommen [HOE13].

Die Anforderungen an den seitlichen Unterfahrschutz basieren auf der Regelung ECE-R 73. Er besteht aus einem oder mehreren Längsträgern mit entsprechenden Verbindungselementen. Die Prüfung erfolgt mittels einer horizontalen, rechtwinklig aufgebrachten statischen Kraft von $1 \mathrm{kN}$. Die maximale Verformung darf am hinteren Ende auf einer Länge von $250 \mathrm{~mm}$ höchstens $30 \mathrm{~mm}$ und für den Rest der Schutzeinrichtung höchstens $150 \mathrm{~mm}$ betragen. Permanent am Fahrzeug angebrachte Komponenten dürfen die Funktion des seitlichen Unterfahrschutzes übernehmen [ECE12].

\subsubsection{Heckunterfahrschutz}

Dem hinteren Unterfahrschutz kommt bei Auffahrunfällen von Personenkraftwagen eine entscheidende Bedeutung zu. Er ist verpflichtend für alle Nutzfahrzeuge mit einem zGG größer 3,5 t (Klassen N2, N3, O2 und O4), sofern der Abstand der hinteren Begrenzung bis zur letzten Hinterachse mehr als $1000 \mathrm{~mm}$ beträgt und das Fahrgestell in seinem hinteren Bereich in unbeladenem Zustand eine lichte Höhe von mehr als $700 \mathrm{~mm}$ über der Fahrbahn aufweist (§ 32b StVZO und Regelung ECE-R 58) [HOE13]. Ausgenommen sind beispielsweise Sattelzugmaschinen.

Der Heckunterfahrschutz besteht aus einem Querträger und entsprechenden Komponenten zur Anbindung an die Längsträger. Die Profilhöhe muss mindestens $100 \mathrm{~mm}$ betragen. Die Prüfung erfolgt rein statisch. Dabei werden jeweils nacheinander eine Kraft F1 von $100 \mathrm{kN}$ oder $50 \%$ des zGG, je nachdem welcher Wert kleiner ist, an zwei Punkten im mittleren Bereich sowie eine Kraft F2 von $50 \mathrm{kN}$ bzw. 25\% des zGG an drei weiteren, über die Länge verteilten Punkten horizontal und rechtwinklig auf den Querträger aufgebracht (Abb. 2-4). Die maximale Durchbiegung in der Prüfung darf 400 mm nicht überschreiten [ECE08]. 


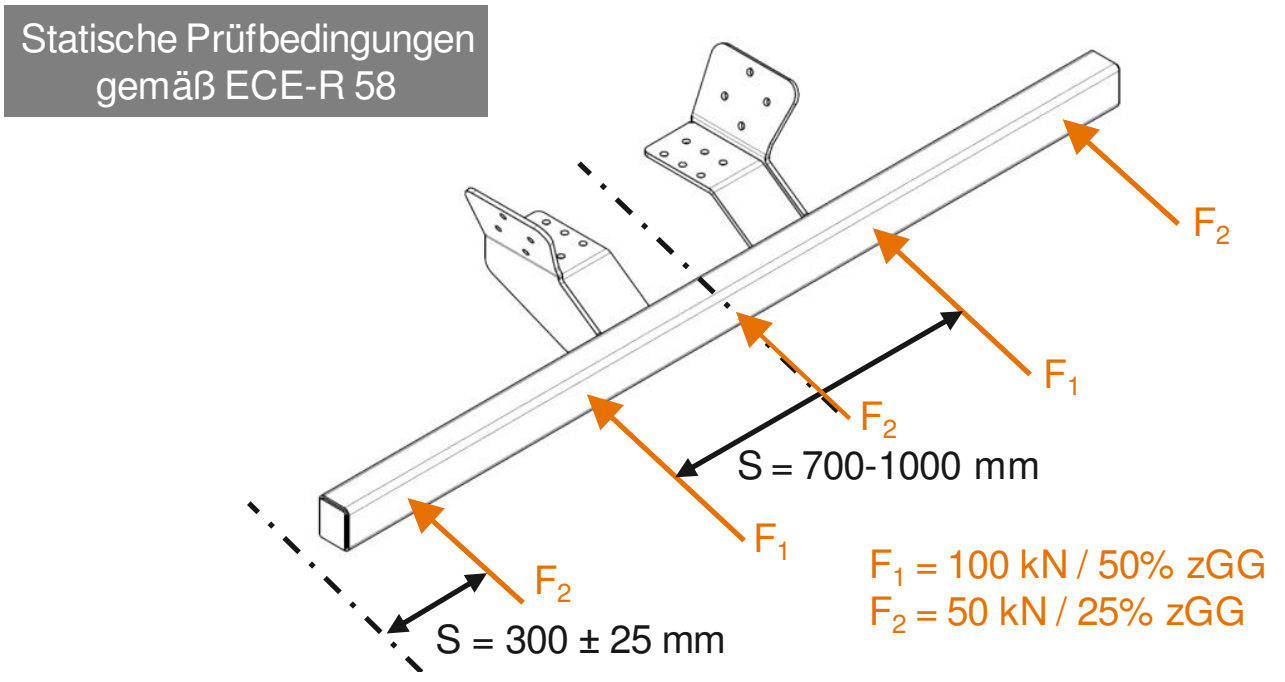

Abb. 2-4: Anforderungen an den hinteren Unterfahrschutz

Wie Realversuche des ADAC zeigen, halten die gemäß der beschriebenen Prüfanforderungen ausgelegten hinteren Unterfahrschutzeinrichtungen einem Pkw-Aufprall, beispielsweise mit $56 \mathrm{~km} / \mathrm{h}$ und einer Überdeckung von 75\%, nicht stand [ADA12]. Eine Änderung der Regelung ECE-R 58 ist mittlerweile in einer Sitzung der UNECE WP 29 im November 2015 beschlossen worden [ECE15], womit die EU in naher Zukunft einen besseren Unterfahrschutz vorschreiben wird.

\subsubsection{Ladungssicherung}

Die Kontrollen des Bundesamts für Güterverkehr (BAG) und der Polizei hinsichtlich Ladungssicherung überprüfen die Einhaltung von DIN- bzw. VDI-Normen. Die VDI-Richtlinie 2700 ist seit 1975 das Grundlagenwerk zur Berechnung von Ladungssicherungskräften und wird laufend weiterentwickelt [VDI04]. Seit 2004 zählt zusätzlich die DIN EN 12195-1:2003 in Deutschland zu den anerkannten Regeln der Technik im Sinne des $\S 22$ der StVO. Sie basiert auf der VDI-Richtlinie 2700, weist aber punktuelle Unterschiede auf, insbesondere beim „Niederzurren“ (bis zu 25\% höhere Vorspannkräfte als in der VDI-Richtlinie). Entsprechend $\S 22$ Absatz 1 der StVO ist die Ladung einschließlich Geräte zur Ladungssicherung sowie Ladeeinrichtungen so zu verstauen, dass sie selbst bei Vollbremsung oder plötzlicher Ausweichbewegung nicht verrutscht, umfällt oder hin- und herrollt [DIN11]. Zusätzlich werden in den Normen DIN EN 12640 und DIN EN 12642 die Beschaffenheit der Zurrpunkte, ihre Anzahl und Position auf der Ladefläche bzw. die Anforderungen an die Fahrzeugaufbauten beschrieben [DIN01] [DIN07].

Das Thema Ladungssicherheit stellte einen zentralen Punkt im Rahmen des ersten Experten-Workshops dar. Nach Aussage eines der Workshopteilnehmer gibt es bezüglich Verkehrsunfälle infolge unzureichend gesicherter Ladung keine allgemeinen statistischen Daten. Ein Grund dafür ist auch, dass zur genauen Ursachenklärung solcher Unfälle eine hohe Expertise seitens der Polizeibeamten erforderlich ist. Die Beurteilung der Ladungssicherung und -verteilung ist häufig komplex und erfordert viel Sachkompetenz. Stattdessen werden in 
der Verkehrsunfallanzeige dann mitunter andere Fehlverhalten wie "Nicht angepasste Geschwindigkeit" angegeben, sodass Fehler hinsichtlich der Ladungssicherung oftmals nicht dokumentiert werden. Im Workshop wird auch angeführt, dass viele Unfälle auf Ausbildungslücken zurückzuführen sind. So werden Zurrgurte falsch bzw. nicht optimal angewendet. Gründe hierfür sind u.a. auch häufige Personalwechsel und sprachliche Barrieren. Mangelnde Ladungssicherung ist kein zeitliches Problem. Vielmehr müsste die gesamte Kette zusammenarbeiten, dies betrifft auch die Verlader. Allgemein bleibt aber die Problematik bestehen, dass der Anteil von Lkw-Unfällen infolge unzureichender Ladungssicherung statistisch schwer erfassbar ist.

In einem bei der BAG geführten Experteninterview wird angeführt, dass ca. $20 \%$ der auf Ladungssicherung geprüften Fahrzeuge beanstandet werden. Generell kann die Lastverteilung kaum überprüft werden. Zudem erfolgen Überladungen häufig unter Einhaltung der Verwarnungsgrenze. Hinsichtlich der Ladungssicherung sind Stückguttransporte, aber auch Fahrzeuge mit seitlicher Schiebeplane (Curtainsider), oft auffällig. In der Kontrollpraxis werden die Fahrzeuge optisch vorselektiert, d.h. die zugehörigen Statistiken hinsichtlich Überladung, technischer Mängel oder Ladungssicherung repräsentieren nicht die realen Anteile.

\subsubsection{Aktive Systeme}

Eine Übersicht über die für Güterkraftfahrzeuge erhältlichen bzw. noch in der Erprobungsphase befindlichen Systeme der aktiven Sicherheit gibt Abb. 2-5, gegliedert in Systeme für den Front- und Heckbereich sowie zum Spurwechseln und Spurhalten.

\section{Fahrspur wechseln / Abbiegen}

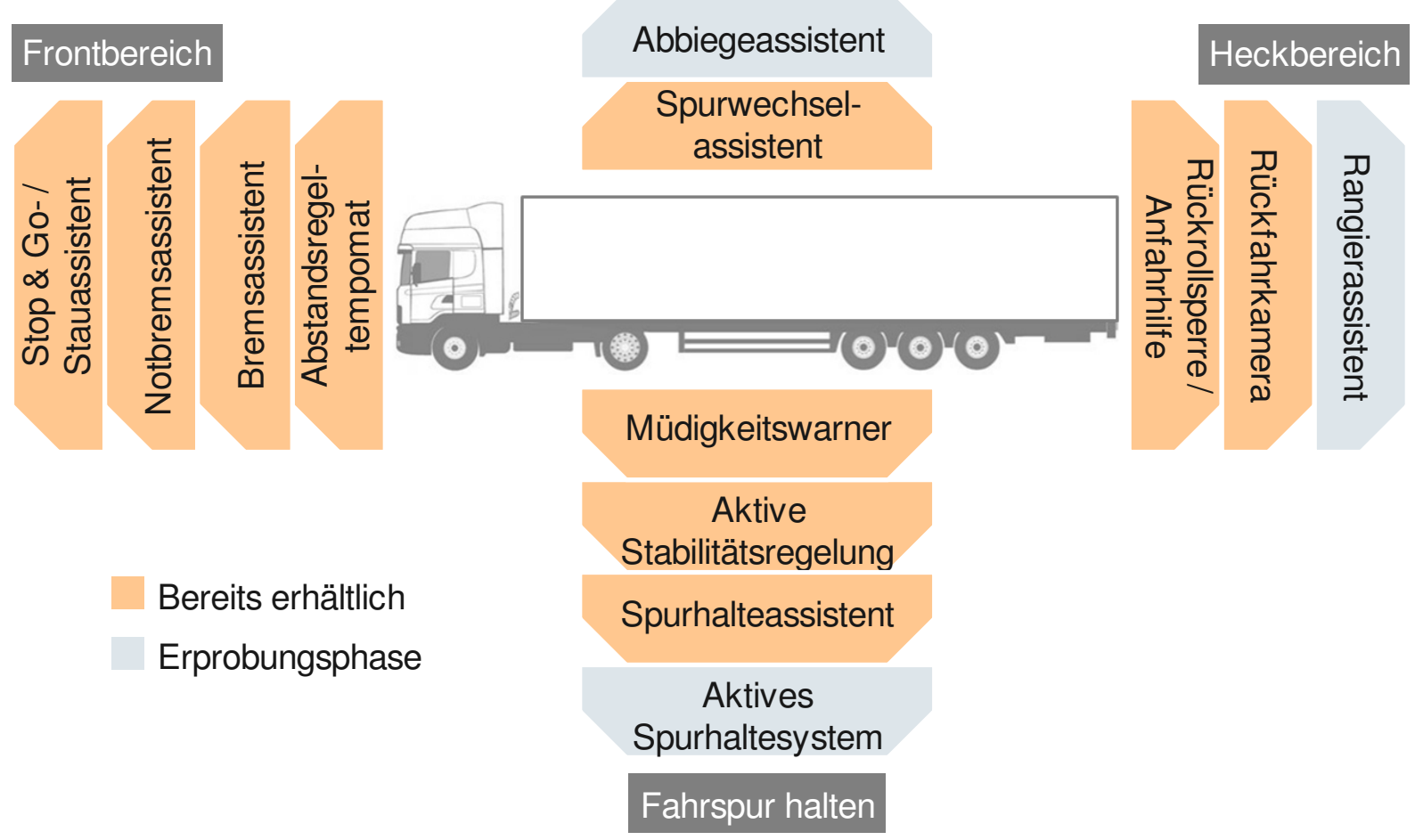

Abb. 2-5: Aktive Sicherheitssysteme für Güterkraftfahrzeuge 
Von den in der Erprobung befindlichen Systemen wird voraussichtlich der Abbiegeassistent, welcher Kollisionen mit ungeschützten Verkehrsteilnehmern beim Rechtsabbiegen vermeiden soll, als erstes System die Serienreife erreichen. Er soll mit der IAA Nutzfahrzeuge im September 2016 eingeführt werden. Einen Ausblick auf das autonome Güterkraftfahrzeug der Zukunft gibt die 2014 vorgestellte Studie „Mercedes-Benz Future Truck 2025“ [RAT14]. Das für den Autobahnverkehr ausgelegte Sattelzugfahrzeug beschleunigt und bremst selbstständig in Abhängigkeit von Strecke, Topografie und Verkehrslage. Die Sensorik ist heute bereits verfügbar. Verschiedene Feldversuche prognostizieren ein hohes Unfallvermeidungspotential von aktiven Systemen. Volvo verfolgt mit dem „Non-Hit car and truck“-Projekt sogar das Ziel, dass 2020 niemand mehr von oder in einem Fahrzeug von Volvo bei einem Unfall getötet wird [BRÜ14]. Dies soll mittels einer $360^{\circ}$-Fahrzeugumgebungsüberwachung durch Einsatz heute bereits verwendeter Sensoren erreicht werden.

Auf Seiten der Gesetzgebung schreibt die EU-Verordnung 661/2009/EC u.a. die europaweite Ausstattung mit verschiedenen Fahrerassistenzsystemen in zeitlicher Staffelung vor. Seit 1. November 2014 sind elektronische Fahrstabilitätsregelsysteme, also ESP (Elektronisches Stabilitätsprogramm) bzw. ESC (Electronic Stability Control), Pflicht für alle neuen Straßenfahrzeuge, d.h. auch Lkw und deren Anhängefahrzeuge. Für neue Typzulassungen gilt dies bereits seit dem 1. November 2011. Spurverlassenswarner (LDW - Lane Departure Warning) und fortschrittliche Notbremssysteme (AEB - Autonomous Emergency Braking) sind seit dem 1. November 2015 für neu zugelassene Lkw mit zGG größer 3,5 t und Bussen mit mehr als neun Sitzplätzen verpflichtend, was auf neue Typzulassungen bereits seit 1. November 2013 zutrifft. Daneben werden verschiedene Maßnahmen zur Verbesserung der Wirtschaftlichkeit und Umweltfreundlichkeit wie Reifendrucküberwachung (TPMS - Tire Pressure Monitoring System), Gangschaltanzeigen und bestimmte Reifeneigenschaften vorwiegend für Pkw vorgeschrieben. Die Verordnung selbst enthält keine Leistungsanforderungen und Ausnahmen, verweist aber für Fahrstabilitätsregelsysteme auf die Bremsenrichtlinie ECE-R 13, während für LDW- und AEB-Systeme die Durchführungsverordnungen 351/2012/EC und 347/2012/EC gelten. Dabei muss für AEB-Systeme ebenfalls die Übereinstimmung mit der Richtlinie für Bremsanlagen (ECE-R 13) nachgewiesen werden [EGV09].

Die Durchführungsverordnung 351/2012/EC schreibt für LDW-Systeme einen Regelbereich oberhalb $60 \mathrm{~km} / \mathrm{h}$ vor. Sobald das Fahrzeug eine sichtbare Fahrbahnmarkierung überfährt, muss eine optische, haptische und/oder akustische Warnung erfolgen. Diese Warnungen sollen durch klare Fahrerhandlungen, bspw. durch Setzen der Fahrtrichtungsanzeige, unterdrückbar sein. Die Implementierung einer Abschaltbarkeit des Systems ist zulässig, allerdings muss die Funktion bei Fahrzeugstart automatisch wieder aktiviert werden [EGV12].

Für AEB-Systeme sind in der Durchführungsverordnung 347/2012/EC verschiedene Anforderungen und Nachweistests für die autonome Notbremsung sowohl auf ein bewegtes als auch auf ein stationäres in der Fahrspur befindliches Fahrzeug vor dem Güterkraftfahrzeug zu erfüllen. Während bei der autonomen Notbremsung auf ein bewegtes Vorausfahrzeug eine Kollision bei griffiger Fahrbahn vermieden werden soll, wird bei "stehenden Zielen“ keine Unfallvermeidung sondern lediglich eine Aufprallschwereminderung verlangt. Dabei 
muss das AEB-System unter Berücksichtigung der Verzögerung während der Warnkaskade ohne Fahrerreaktion eine Geschwindigkeitsreduzierung um mindestens $10 \mathrm{~km} / \mathrm{h}$, d.h. von $80 \mathrm{~km} / \mathrm{h}$ auf unter $70 \mathrm{~km} / \mathrm{h}$, bewirken, bevor es zur Kollision kommt. Analog zu den Anforderungen für ein bewegtes Vorausfahrzeug darf die autonome Notbremsung hier nicht früher als 3,0 s vor der Kollision erfolgen. Die vorgegebene Mindestreduktion der Geschwindigkeit entspricht einer Minderung der Kollisionsenergie um 23\%. Der in zweiter Stufe ab 2016/2018 (Typzulassungen / Neuzulassungen) geforderte Geschwindigkeitsabbau um insgesamt mindestens $20 \mathrm{~km} / \mathrm{h}$, also von 80 auf unter $60 \mathrm{~km} / \mathrm{h}$, entspricht einer Energieminderung um 44\%. Diese (Mindest-)Forderungen sind in Anbetracht des heutigen Standes der Technik als vergleichsweise gering einzustufen, auch weil allein schon während der Warnphase durch Teilbremsung oder Bremsruck eine Geschwindigkeitsreduktion um $15 \mathrm{~km} / \mathrm{h}$ zulässig ist [PET13].

Einige Fahrzeugarten sind, u.a. auch aufgrund der Ergebnisse einer von der EU beauftragten Kosten-/Nutzen-Analyse des englischen Transport Research Laboratory (TRL), von der verpflichtenden Ausstattung mit AEB- und LDW-Systemen ausgenommen. Dazu zählen Sattelzugmaschinen mit zGG größer $8 \mathrm{t}$ („Wochenmarktfahrzeuge“), Gelenkbusse und Busse mit mehr Stehplätzen (unter 23) als Sitzplätzen, Gelände- und Spezialfahrzeuge (gemäß Richtlinie 2007/46/EG, Anhang II, Teil A, Absätze 4.2, 4.3 und 5) sowie Omnibusse und Lkw mit mehr als drei Achsen [PET13].

\subsubsection{Manipulationen an Einrichtungen zur Lenkzeitdokumentation}

Im Rahmen des ersten Experten-Workshops wurde geäußert, dass Manipulationen am digitalen Fahrtenschreiber und an Steuergeräten ein Problem und gerade bei Fahrzeugen osteuropäischer Betreiber häufiger anzutreffen sind. Dadurch kann es zu Fehlfunktionen von Fahrerassistenzsystemen und damit zu Unfällen kommen. Laut einem Interview bei der BAG sind Manipulationen technisch aber auch in Bezug auf den Fahrer sehr schwer nachzuweisen. Die in der Vergangenheit festgestellten Manipulationen, beispielsweise mittels eines Magneten am sogenannten Kitas-Geber, einem Geschwindigkeitssensor am Getriebe, sind heutzutage kaum noch zu verzeichnen.

Weitere Maßnahmen zur Umgehung der gesetzlich vorgeschriebenen Ruhezeiten sind auch in Bezug auf die Fahrerkarte festzustellen. Dazu zählen die Verwendung einer zweiten Fahrerkarte bzw. das Fahren ohne Fahrerkarte oder das Abdecken des integrierten Chips. Aber es werden auch Manipulationen an den aufgezeichneten Daten durchgeführt, beispielsweise durch Veränderung der Signaturinformationen oder Löschung der Daten [KIM13].

\section{$2.2 \quad$ Leicht-Lkw}

Bei Leicht-Lkw handelt es sich um Gliederzüge für den Volumenverkehr mit extrem leichter Bauweise und einem zGG von 11,99 t, weshalb sie im Weiteren auch als $12 \mathrm{t}$-Leicht-LkwKombinationen bezeichnet werden. Kombiniert wird ein 7,49 t-Motorwagen mit einem meist einachsigen 4,5 t-Zentralachsanhänger, wodurch insgesamt eine Nutzlast bis zu $5 \mathrm{t}$ möglich ist (Abb. 2-6). Äußerlich charakteristisch im Vergleich zum 40 t-Gliederzug sind das kleinere Fahrerhaus und das reduzierte Fahrgestell. Bis Oktober 2015 waren diese Fahrzeugkombi- 
nationen von der 2005 eingeführten Mautpflicht für Lkw ab $12 \mathrm{t}$ auf Fernstraßen ausgenommen, welche seither auch für Fahrzeuge und Kombinationen ab 7,5 $\mathrm{t}$ gilt. Problematisch ist in erster Linie die hohe Windanfälligkeit der Leicht-Lkw-Kombinationen, die vollbeladen und bei gleichen Abmessungen immer noch ca. $3 \mathrm{t}$ weniger wiegen als übliche $40 \mathrm{t}$-Gliederzüge im leeren Zustand.

\section{0 t-Standard-Gliederzug}

\section{2 t-Leicht-Lkw-Kombination}
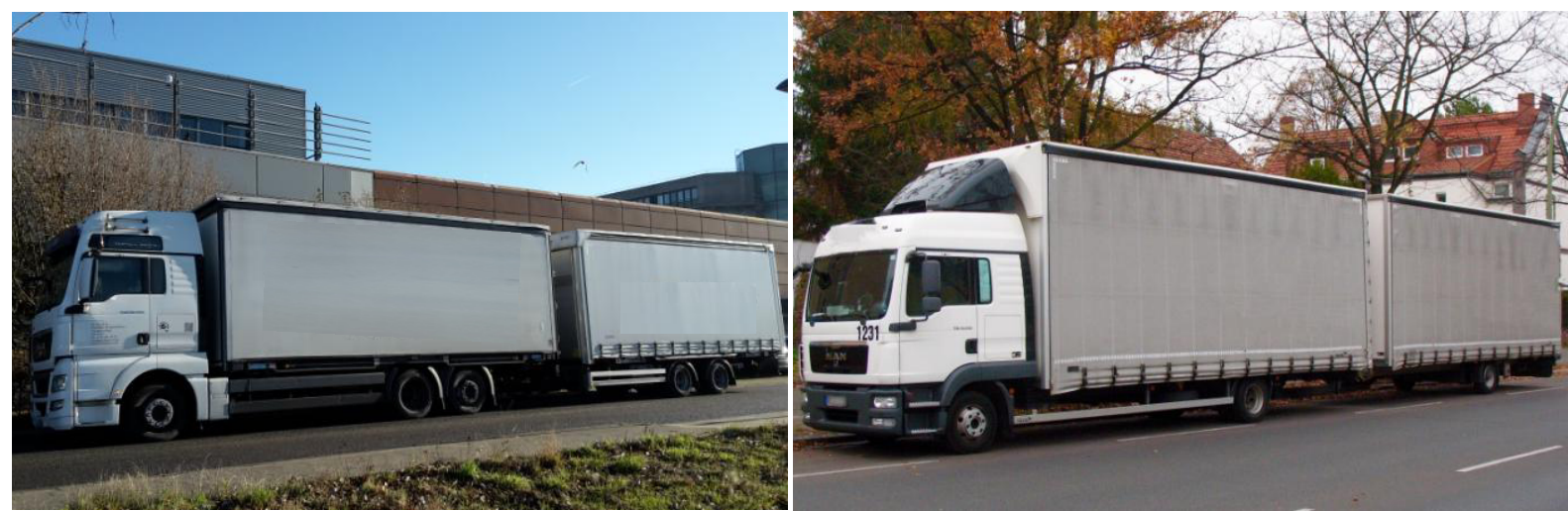

\begin{tabular}{|c|c|c|c|}
\hline & 40 t-Standard-Gliederzug & \multicolumn{2}{|c|}{12 t-Leicht-Lkw-Kombination } \\
\hline Ladevolumen & $120 \mathrm{~m}^{3}$ & $120 \mathrm{~m}^{3}$ & $110 \mathrm{~m}^{3}$ \\
\hline Leergewicht & $15 t$ & \multicolumn{2}{|c|}{$7 t$} \\
\hline Zuladung & bis $25 \mathrm{t}$ & \multicolumn{2}{|c|}{ bis $5 t$} \\
\hline Länge & bis $18,75 \mathrm{~m}$ & \multicolumn{2}{|c|}{ bis $18,75 \mathrm{~m}$} \\
\hline Höhe & $4 \mathrm{~m}$ & \multicolumn{2}{|c|}{$4 \mathrm{~m}$} \\
\hline Laderaumhöhe & $3,1 \mathrm{~m}$ & $3,1 \mathrm{~m}$ & $3,0 \mathrm{~m}$ \\
\hline Anzahl Achsen & 5 & 3 & 4 \\
\hline \multirow{2}{*}{$\begin{array}{l}\text { Ladelängen (Zug- } \\
\text { fzg. / Anhänger) }\end{array}$} & \multirow{2}{*}{$7,75 \mathrm{~m} / 7,75 \mathrm{~m}$} & $7,75 \mathrm{~m} / 7,75 \mathrm{~m}$ & \multirow{2}{*}{$6,60 \mathrm{~m} / 8,20 \mathrm{~m}$} \\
\hline & & $6,45 \mathrm{~m} / 9,05 \mathrm{~m}$ & \\
\hline
\end{tabular}

Abb. 2-6: 12 t-Leicht-Lkw-Kombinationen im Vergleich zum 40 t-Standard-Gliederzug

Im Zuge des Wegfalls der Mautbefreiung werden für eine 3-achsige Leicht-Lkw-Kombination in Euro-6-Ausführung derzeit 11,3 Cent je Kilometer auf Autobahnen sowie auf mautpflichtigen Bundesstraßen fällig. Dennoch stocken einige der Logistikdienstleister ihren Leicht-LkwFuhrpark weiter auf und haben vereinzelt auch einen eigenen Vertrieb für diese Fahrzeuge aufgebaut. Nach Angaben eines Betreibers ist der Verbrauch bzw. der $\mathrm{CO}_{2}$-Ausstoß im Vergleich zu den 40 t-Standard-Gliederzügen mit gleichem Ladevolumen um 30 bis $40 \%$ niedriger. Inzwischen liege der Verbrauch bei unter 20 Litern auf $100 \mathrm{~km}$. Maßgeblich hierfür seien das geringe Eigengewicht sowie eine verbesserte Aerodynamik durch den Einsatz von Spoilern. Vor allem aufgrund dieser deutlichen Reduzierung des Kraftstoffverbrauchs würden die Betriebskosten um 10 bis $20 \%$ niedriger ausfallen, wodurch die 12 t-Leicht-Lkw-Kombinationen aus Sicht der Logistikdienstleister nachwievor wirtschaftlich seien [RAT15]. 
Durch Auflastung der Fahrzeuge um $2 \mathrm{t}$, was eine Erhöhung der Nutzlast auf $7 \mathrm{t}$ ermöglicht, soll die Effizienz weiter gesteigert werden. Auch kann das Transport-Portfolio dadurch deutlich erweitert werden, d.h. es können fortan andere Güter als Dämmstoffe, leere IBC-Container (kubische Kunststoffbehälter mit Metallaußengestell für flüssige / rieselfähige Stoffe) oder Verpackungsmaterialien mit diesen Fahrzeugen transportiert werden. Durch $2 \mathrm{t}$ mehr Nutzlast wird z.B. der Einsatz für die Automobilindustrie erleichtert [RAT15]. Während die ersten $12 \mathrm{t}$-Leicht-Lkw-Kombinationen ausschließlich hinsichtlich $5 \mathrm{t}$ Nutzlast ausgelegt waren, handelt es sich bei den neueren Varianten um schwerere Fahrzeuge, die zulassungsrechtlich abgelastet werden und so eine höhere Nutzlast von $7 \mathrm{t}$ transportieren können. Dadurch kann nun z.B. auch das Dosen-Segment bedient werden, was mit den bisherigen Kombinationen nicht möglich gewesen ist. Auch im Bereich der Dämmmaterialien gibt es laut Branche viele Ladungen zwischen 5 und 7 t, etwa Glaswolleprodukte. Leicht-Lkw sind jedoch keine Nahverkehrs-Lkw. Sie können ihre Betriebskostenvorteile nur auf langen Strecken ausspielen, wo sie trotz Maut immer noch deutlich günstiger sind als schwere Lkw. Zudem hat es im Ausland hinsichtlich der Maut keine Veränderungen gegeben. So bleibt beispielsweise der Transit durch die Schweiz in Richtung Italien nachwievor deutlich günstiger [LAU15].

Im Rahmen eines Experteninterviews bei einer Speditionsgesellschaft erläuterte einer der Geschäftsführer seine Sicht auf das Thema Leicht-Lkw näher: Nach Einschätzung vieler Branchenexperten wurde aus verschiedenen Gründen eine gewinnbringende Nutzung der 12 t-Leicht-Lkw-Kombinationen auf breiter Front zunächst als nicht möglich angesehen. So galt eine Nutzlast von unter $5 \mathrm{t}$ für viele Volumengüter als zu gering und es wurde von einer unzureichenden Auslastung mit hohem Leerkilometeranteil ausgegangen. Vor dem Hintergrund der Einführung des digitalen Tachos und der damit verbundenen genaueren Erfassung bzw. Kontrolle der Lenk- und Ruhezeiten wurde insgesamt von einer unzureichenden Ausnutzung der Arbeitszeiten der Fahrer ausgegangen. Zudem nahm man an, dass es schwierig werden würde, für diese Fahrzeuge Fahrer zu finden. Nicht nur aufgrund des vorherrschenden Fahrermangels, sondern auch aufgrund des fehlenden Fahr- und Wohnkomforts sowie des geringen Prestiges der 12 t-Leicht-Lkw-Kombinationen.

Erst die Überschreitung gesetzlicher Grenzen (zGG, Abmessungen, Lenk- \& Ruhezeiten) einiger Vorreiter habe aus Sicht der Speditionsgesellschaft zum Erfolg der Leicht-Lkw geführt. Auch wurde von den Branchenexperten bezüglich der Fahrerproblematik nicht der überwiegende Einsatz von Fahrern mit altem Pkw-Führerschein (ausgestellt bis 1.1.1999) berücksichtigt, die diese Gespanne ohne separaten Lkw-Führerschein fahren dürfen. Als Folge der fehlenden Fahrprüfung für Lkw bzw. der fehlenden Fahrerfahrung mit den gegebenen Abmessungen hätten Fahrer von Leicht-Lkw häufig Probleme, diese souverän und ohne Verursachung von Sachschäden zu bewegen, insbesondere an Rampen und Verladestellen. Durch das zunehmende Aufkommen der Leicht-Lkw sei das Volumensegment in einen Leicht- und einen Schwervolumentransportmarkt aufgespalten worden.

Die einzelnen Varianten bzw. Ladelängenaufteilungen zwischen Zugfahrzeug und Anhänger der 12 t-Leicht-Lkw-Kombinationen (Abb. 2-6) resultieren laut der befragten Speditionsgesellschaft aus Vorgaben der Verlader, die häufig metrische Abmessungen der Fahrzeuge zum 
Transport ihrer Güter benötigen. Ein weiterer Grund ist der Wunsch nach einer hohen Wendigkeit des Zugfahrzeugs. Das Leergewicht der Anhänger liegt häufig bei ca. 2 t. Daher sind diese, insbesondere unbeladen, deutlich anfälliger für Seitenwind als das Zugfahrzeug. Die seitens eines Betreibers angekündigte Auflastung der Fahrzeuge um $2 \mathrm{t}$ ist nur für eine Längenaufteilung von 6,45 m / 9,05 m sinnvoll, da nur hier der Anhänger ein deutlich höheres Ladevolumen besitzt. Allerdings wird dabei die Seitenwindanfälligkeit aufgrund der größeren Windangriffsfläche bei nahezu unverändertem Anhängerleergewicht eher noch größer. Andere Aufteilungen würden zu einer Überladung des Zugfahrzeugs führen, sofern dieses nicht aufgelastet wird. Bei einer Auflastung des Zugfahrzeuges wäre jedoch der alte PkwFührerschein nicht mehr ausreichend.

Zur Reduzierung der Kippgefahr unter Seitenwind setzen einige Logistikdienstleister neuerdings sogenannte Sturmstützen an den Anhängern ihrer Leicht-Lkw-Kombinationen ein. Dabei handelt es sich um ein Profil, welches im vorderen unteren Anhängerbereich angebracht ist und außen, unmittelbar hinter dem seitlichen Unterfahrschutz, jeweils über entsprechende Stützfüße mit schräger Auflagefläche verfügt (Abb. 2-7). Befindet sich der Anhänger nahe der Kippgrenze, setzt der jeweilige Fuß auf dem Boden auf und soll durch die damit verbundene Stützwirkung ein Kippen verhindern helfen.

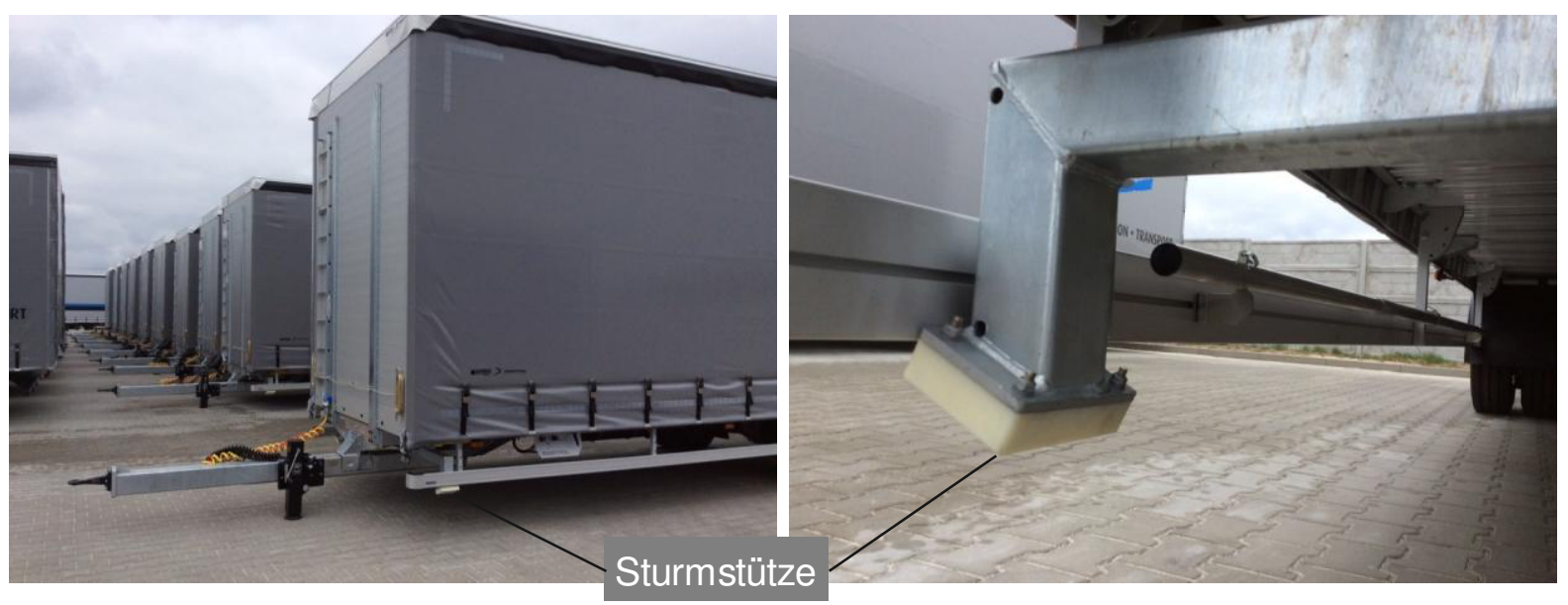

Abb. 2-7: Implementierung von Sturmstützen an Leicht-Lkw-Anhängern [KEM15]

Die Wirksamkeit der Sturmstützen ist, abgesehen von statischen Kippversuchen, bislang nicht getestet worden. Um diesbezüglich weitere Erkenntnisse zu erlangen, wird im Rahmen der Simulationen zur Kippstabilität bei Seitenwind in Kapitel 5 auch die Implementierung von Sturmstützen berücksichtigt.

Analysen zum Verkehrsaufkommen und Unfallgeschehen von Leicht-Lkw werden in Kapitel 3.3 und Kapitel 4.2 behandelt. 


\section{$3 \quad$ Analyse des Lkw-Verkehrs}

Ausgangspunkt der Unfallanalyse in Kapitel 4 bildet zunächst eine Analyse des Verkehrsaufkommens mit Lkw über 3,5 t zGG in Deutschland. Dabei erfolgt eine Differenzierung nach Gewichtsklassen und sofern möglich auch nach Fahrzeugtyp. Es werden Aspekte wie Bestand, Fahrleistung, Verkehrsräume und durchschnittliche Nutzungsdauer berücksichtigt.

Neben diesen rein verkehrsspezifischen Gesichtspunkten werden auch sicherheitsrelevante Aspekte in Bezug auf das Fahrerverhalten, die Fahrzeugwartung und die Arbeitsbedingungen betrachtet. Bestandteil der Analyse sind auch die geltenden Sozialvorschriften im StraBenverkehr, insbesondere die Regelung der Lenk- und Ruhezeiten.

Abschließend wird das Verkehrsaufkommen von Leicht-Lkw näher analysiert. Hierzu sind Zählungen an drei für den Güterverkehr bedeutenden Knotenpunkten in Westdeutschland durchgeführt und ausgewertet worden.

\section{1 Übersicht Straßengüterverkehr}

Im Folgenden soll eine kurze Übersicht über den Gütertransport in Deutschland gegeben werden. Dies umfasst neben Auswertungen zum Fahrzeugbestand und zu Verkehrsaufkommen bzw. Fahrleistung auch Daten aus der Kontrollstatistik des Bundesamts für Güterverkehr (BAG).

\subsubsection{Fahrzeugbestand und -alter}

Aus Abb. 3-1 geht der Bestand an Güterkraftfahrzeugen in Deutschland mit zGG größer 3,5 t jeweils zum 1. Januar der Jahre 2010 bis 2015 hervor. Es wird deutlich, dass dieser für den betrachteten Zeitraum weitestgehend stabil ist, mit einer Gesamtzahl von 713.196 Fahrzeugen im Jahr 2015. Dabei nimmt die Zahl der Liefer- und Lastkraftwagen bis 7,5 t etwas $\mathrm{ab}$, während sich für die anderen Fahrzeuggruppen eine steigende Tendenz zeigt. Der im Jahr 2012 vor allem für Liefer- \& Lastkraftwagen mit einem zGG größer $16 \mathrm{t} \mathrm{zu}$ beobachtende Sprung lässt sich auf eine Änderung in der Statistik zurückführen. Ab hier werden Lastkraftwagen mit Spezialaufbau nicht mehr separat geführt sondern den Liefer- und Lastkraftwagen mit Normalaufbau zugeschlagen, was die Zahl der Fahrzeuge über $16 \mathrm{t} \mathrm{zGG} \mathrm{auf}$ ca. 180.000 und damit auf das Niveau der Sattelzugmaschinen ansteigen lässt. Die in diesem Projekt nicht betrachtete und entsprechend in Abb. 3-1 auch nicht aufgeführte Gruppe der Liefer- und Lastkraftwagen bis 3,5 t zGG wächst stetig und ist mit einer Anzahl von über 2,1 Millionen Fahrzeugen im Jahr 2015 (2010: 1,85 Millionen) viermal so groß wie die Gruppe der Liefer- und Lastkraftwagen mit einem zGG größer 3,5 t.

Das Durchschnittsalter aller Liefer- und Lastkraftwagen in Deutschland betrug zum 1. Januar 2015 7,7 Jahre, während Sattelzugmaschinen im Schnitt nur 4,4 Jahre alt waren [KBA15a]. In Abb. 3-2 wird das Fahrzeugalter für Liefer- und Lastkraftwagen mit einem zGG größer 3,5 t genauer aufgeschlüsselt. Man erkennt, dass die Lkw bis 7,5 t zGG den größten Anteil älterer Fahrzeuge aufweisen. 


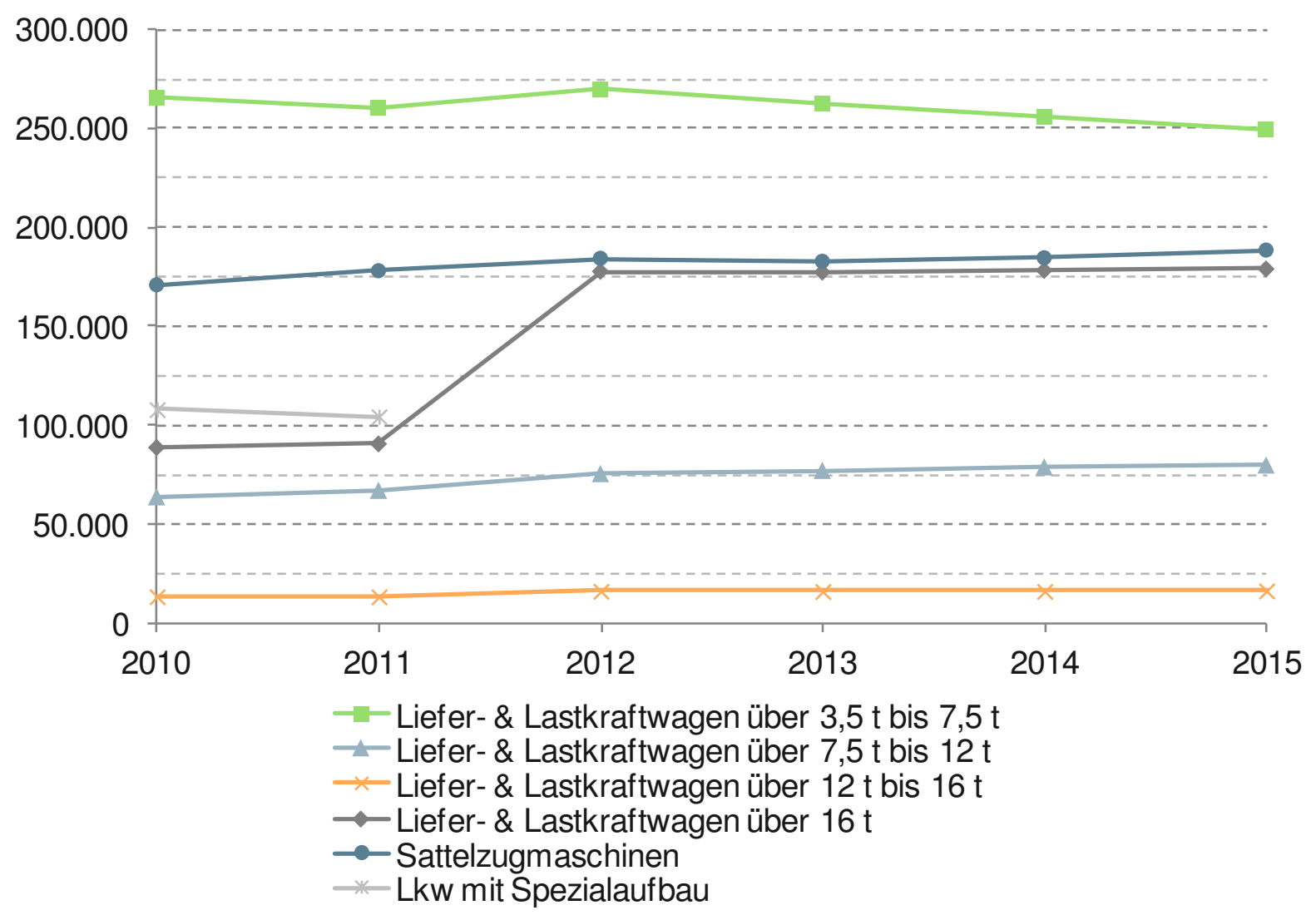

Abb. 3-1: Bestandsentwicklung an Güterkraftfahrzeugen mit zGG größer 3,5 t bis 1. Januar 2015 in Deutschland [STA15] [KBA15a]

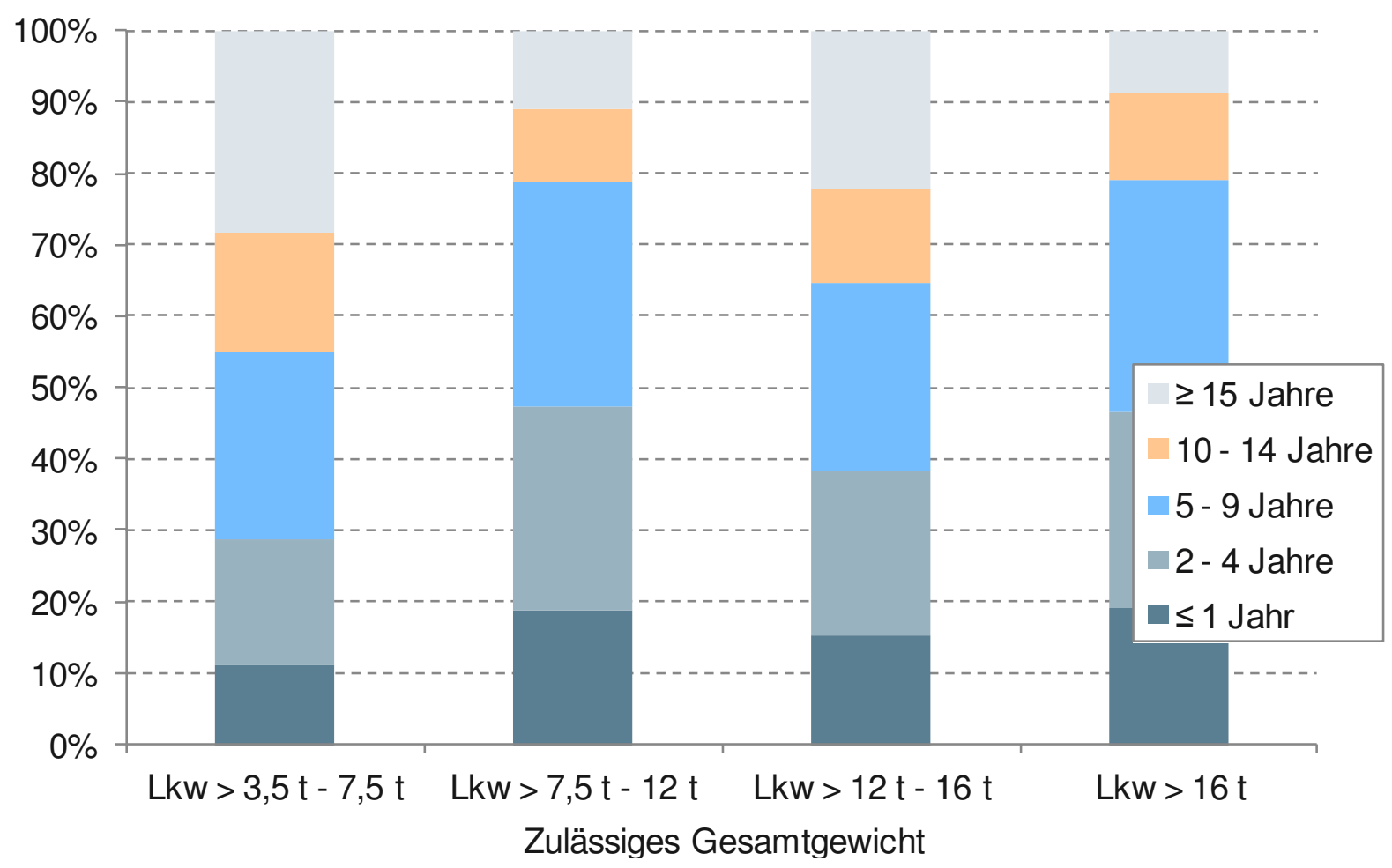

Abb. 3-2: Alter der Liefer- \& Lastkraftwagen mit zGG größer 3,5 t in Deutschland zum 1. Januar 2015 [KBA15a] 
Eine Übersicht zum Alter von Kraftfahrzeuganhängern mit einem zGG größer $4 \mathrm{t}$ geht aus Abb. 3-3 hervor. Bei den Anhängern bis $16 \mathrm{t} z \mathrm{GG}$ hat mehr als die Hälfte ein Alter von mindestens 15 Jahren. Der Altersdurchschnitt nimmt zu höheren Gewichtsklassen hin ab. So beträgt der Anteil von Anhängern mit einem Alter von mindestens 15 Jahren bei einem zGG größer $26 \mathrm{t}$, was fast ausschließlich auf Sattelauflieger zutrifft, nur noch 11\%. Mehr als 76\% der Anhänger mit zGG größer $26 \mathrm{t}$ sind höchstens neun Jahre alt, wobei über $40 \%$ ein Alter von höchstens vier Jahren aufweisen.

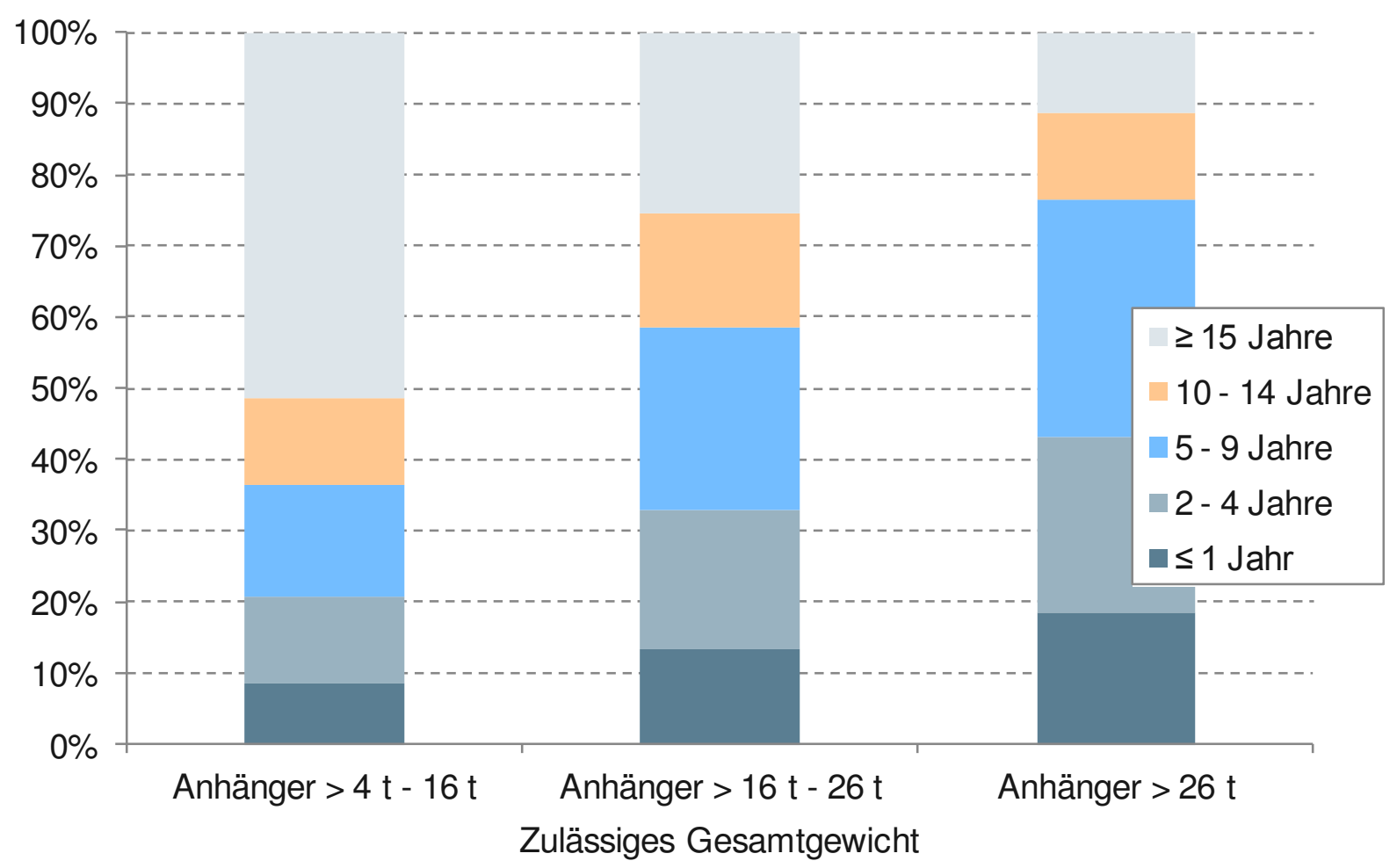

Abb. 3-3: Alter der Kraftfahrzeuganhänger mit zGG größer $4 \mathrm{t}$ in Deutschland zum 1. Januar 2015 [KBA15a]

\subsubsection{Verkehrsaufkommen}

Aus Abb. 3-4 gehen die Anteile der jeweiligen Einsatzbereiche deutscher Güterkraftfahrzege in Bezug auf die Gesamttransportmenge in den Jahren 2010 bis 2014 hervor. Für diese Datenerhebung werden seitens des Kraftfahrt-Bundesamtes (KBA) nur Lastkraftwagen mit mehr als 3,5 t Nutzlast sowie Sattelzugfahrzeuge berücksichtigt. Man stellt fest, dass mehr als $50 \%$ der Transportmenge im Nahverkehr, d.h. in einem Entfernungsbereich bis $50 \mathrm{~km}$, befördert wird. Kabotagetransporte, also Transportdienstleistungen innerhalb eines anderen Landes, werden von deutschen Güterkraftfahrzeugen kaum getätigt [KBA11-15].

In Abb. 3-5 wird die Entfernung, die deutsche Güterkraftfahrzeuge (Nutzlast $>3,5 t$ ) in den Jahren 2010 bis 2014 sowohl mit als auch ohne Ladung zurückgelegt haben nach Fahrzeugart sowie nach zGG aufgeschlüsselt dargestellt. Es wird ersichtlich, dass mehr als die Hälfte der in diesem Zeitraum erzielten Fahrleistung von 147 Milliarden km auf Sattelzüge entfällt 
(Abb. 3-5 links), während Gliederzüge und Sololastkraftwagen vergleichbare Anteile aufweisen. Gliedert man die Daten hinsichtlich des zGG der Güterkraftfahrzeuge (Abb. 3-5 rechts), so entfällt $77 \%$ der Fahrleistung auf schwere Fahrzeuge mit einem zGG größer 30 t.

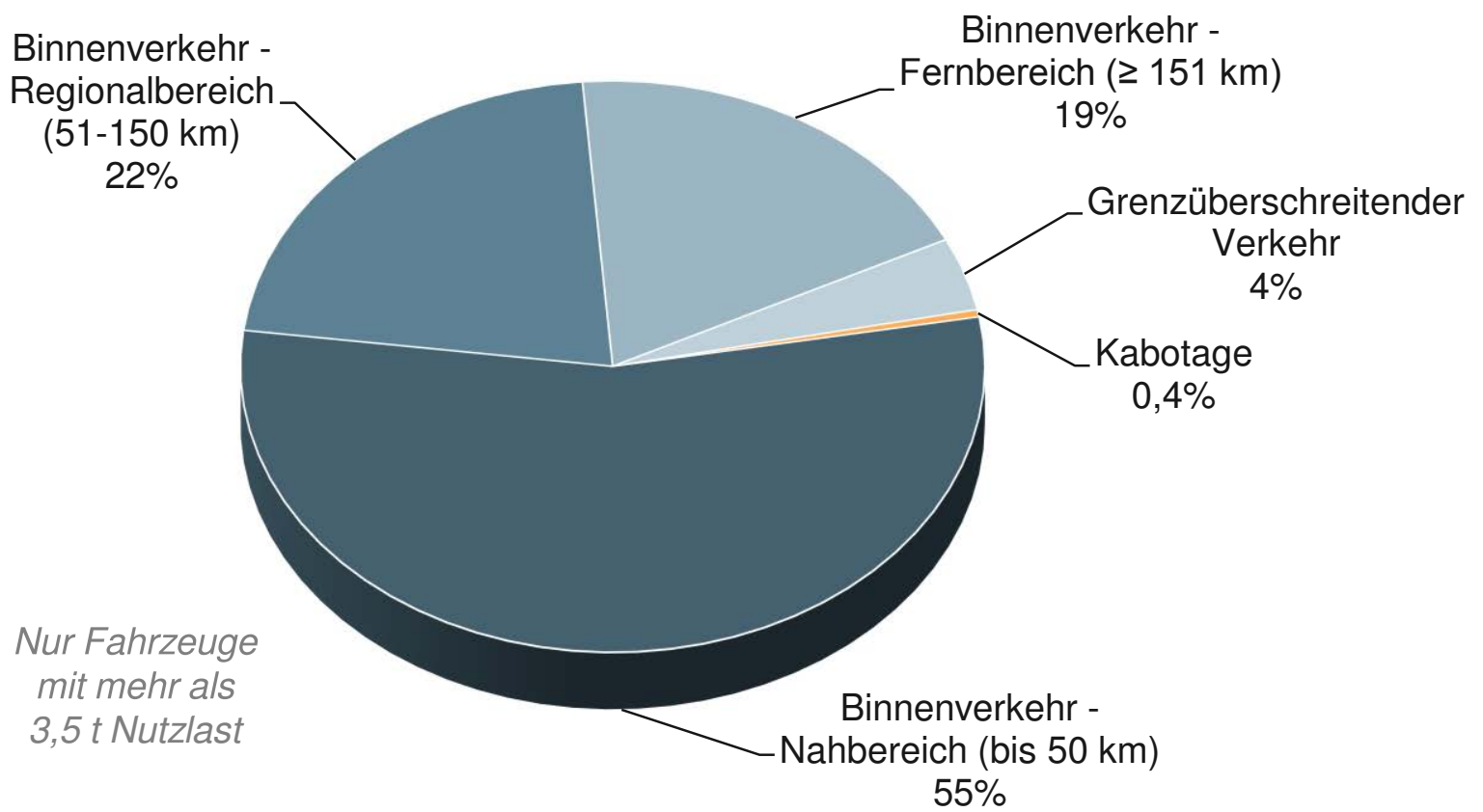

Abb. 3-4: Gütertransport deutscher Lastkraftfahrzeuge nach Einsatzbereich für die Jahre 2010 bis 2014 (Gesamttransportmenge: 14,6 Mrd. Tonnen) [KBA11-15]

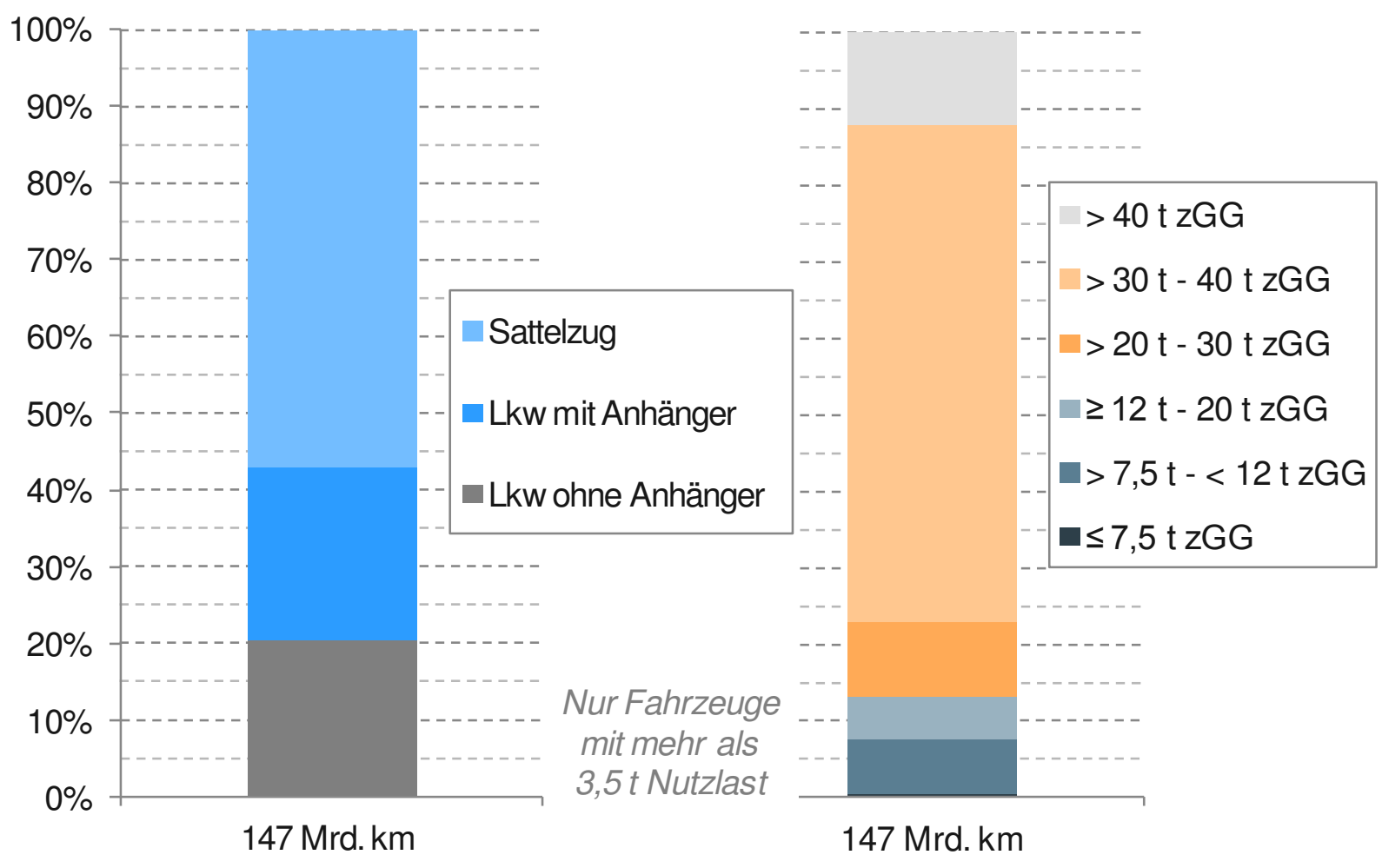

Mit \& ohne Ladung zurückgelegte Entfernung (2010 - 2014)

Abb. 3-5: Fahrleistung deutscher Güterkraftfahrzeuge (2010 - 2014) [KBA11-15] 


\subsubsection{Kontrollstatistik}

Die Kontrolle des Straßengüterverkehrs bezüglich Sozialvorschriften, Ladungssicherung sowie technischer Rahmenbedingungen erfolgt neben der Polizei primär durch das Bundesamt für Güterverkehr (BAG). Die mobile Kontrolltätigkeit des BAG kann in sogenannte Straßenkontrollen nach dem Güterkraftverkehrsgesetz sowie in Mautkontrollen untergliedert werden. Des Weiteren führt das Bundesamt Betriebskontrollen der Bereiche Marktzugang und Maut durch [BAG16].

Die Betriebskontrollen im Bereich Marktzugang dienen zur Überwachung der Einhaltung der Vorschriften des Güterkraftverkehrsgesetzes, die maßgebliche Voraussetzung für den Marktzugang sind. Im Bereich Maut steht die Einhaltung der Bestimmungen des Bundesfernstraßenmautgesetzes (BFStrMG) im Vordergrund. Grundlage für die Durchführung von Betriebskontrollmaßnahmen sind primär die bei Straßenkontrollen getroffenen und anhand eines Kontrollberichts dokumentierten Beanstandungen. Im Bereich Maut können zusätzlich mittels der Brückenkontrollen festgestellte Mehrfachverstöße des Transportunternehmens als Anlass dienen. Insgesamt werden etwa 30 Betriebskontrolleurinnen und -kontrolleure in sieben Prüfgebieten eingesetzt [BAG16].

Die Maut-Straßenkontrollen dienen zur Überprüfung der Einhaltung der Autobahnmaut für Lkw auf Bundesautobahnen und ausgewiesenen Bundesstraßen. Die Mautkontrolle wird einerseits während der Vorbeifahrt des Kontrollfahrzeugs an einem Lkw und andererseits mittels entsprechender Kontrollgeräte am Fahrbahnrand durchgeführt. Desweiteren wird die ordnungsgemäße Mautentrichtung bei Durchfahrt einer der 300 Mautbrücken durch den Betreiber des Mautsystems, Toll Collect, überprüft.

Im Rahmen der Straßenkontrollen nach dem Güterkraftverkehrsgesetz wird die Einhaltung einer Vielzahl an Vorschriften überprüft. Hauptziel ist die Erhöhung der Verkehrssicherheit auf deutschen Straßen. Die Kontrolleure des BAG sind dabei befugt, in- und ausländische Kraftfahrzeuge zur Güterbeförderung sowie Omnibusse anzuhalten und zu kontrollieren. In Abb. 3-6 ist eine Übersicht der in 2014 vom BAG kontrollierten Fahrzeuge dargestellt. Von den mehr als 500.000 überprüften Fahrzeugen waren über $60 \%$ im Ausland zugelassen. Etwa 53\% aller ermittelten Verstöße entfielen dabei aber auf in Deutschland zugelassene Fahrzeuge. Über alle Fahrzeuge gesehen lag die Beanstandungsquote nur bei ca. $43 \%$. Entsprechend war diese für deutsche Fahrzeuge mit $57 \%$ deutlich höher als für ausländische Fahrzeuge (34\%) [BAG16].

In Abb. 3-7 sind die festgestellten Verstöße nach Rechtsgebieten unterteilt dargestellt. Mehr als zwei Drittel der Verstöße entfallen dabei auf das Fahrpersonalrecht. Darunter sind u.a. die nachfolgend in Kapitel 3.2 detailliert erläuterten Lenk-, Ruhe- und Arbeitszeiten zusammengefasst. Bei den deutschen Fahrzeugen ist der Anteil mit über $83 \%$ deutlich höher als bei den ausländischen Fahrzeugen (62\%). Mit einem Anteil von knapp 18\% folgen Verstöße gegen das Straßenverkehrsrecht, z.B. Geschwindigkeitsüberschreitungen. Für die deutschen Fahrzeuge ergibt sich hier ein Anteil von ca. $9 \%$, für die ausländischen von ca. $27 \%$. Die 
übrigen Rechtsgebiete liegen alle jeweils im niedrigen einstelligen Prozentbereich und sind somit von untergeordneter Bedeutung [BAG16].

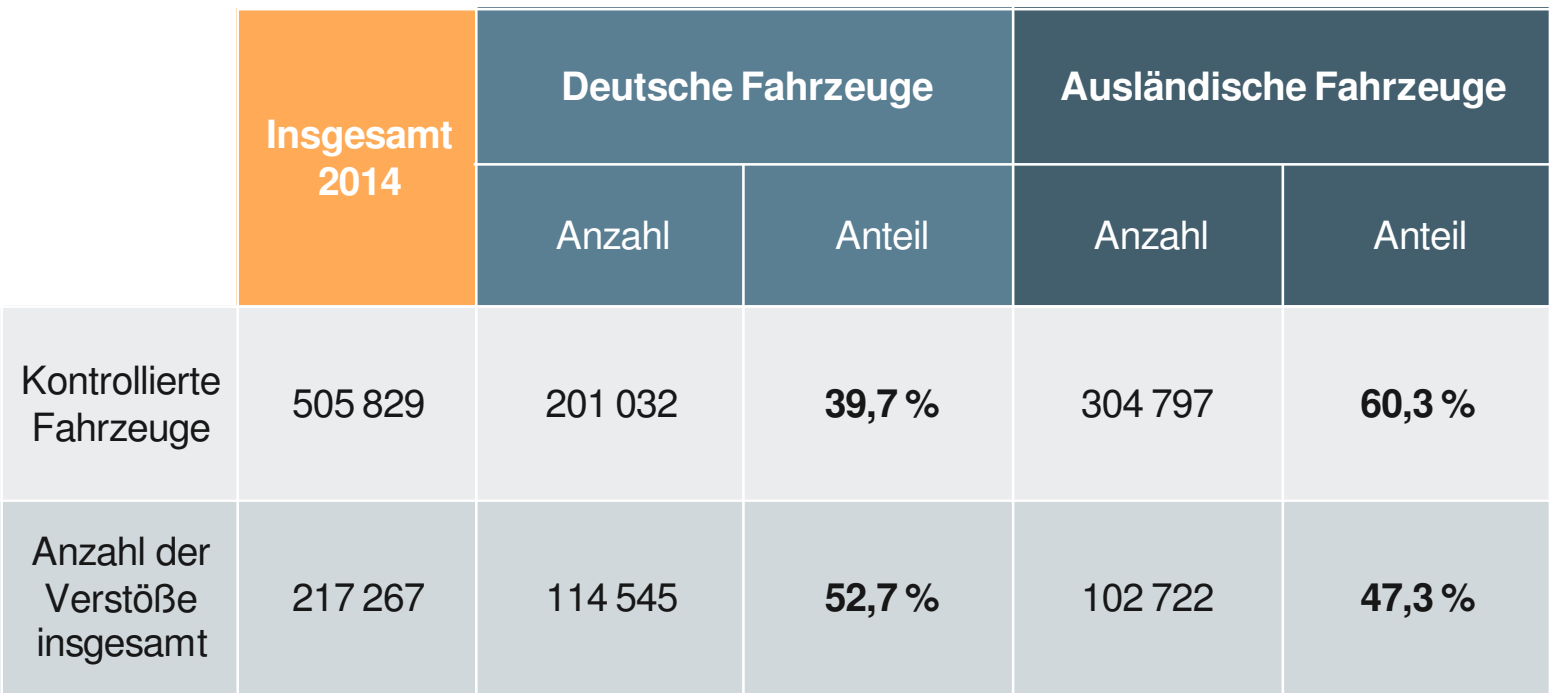

Abb. 3-6: Übersicht der in 2014 kontrollierten Fahrzeuge sowie deren Verstöße differenziert nach Zulassungsland [BAG16]

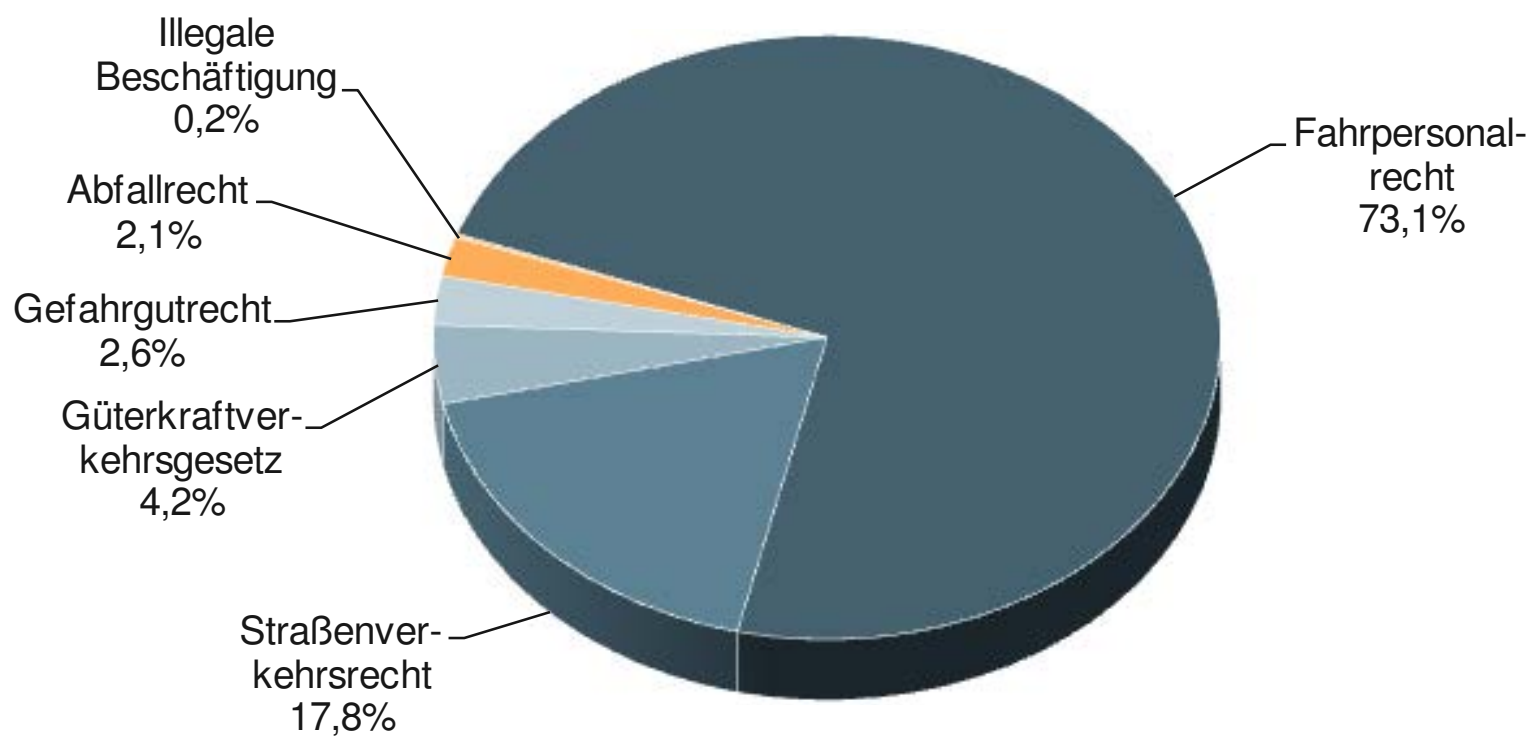

Abb. 3-7: In 2014 bei Straßenkontrollen festgestellte Verstöße nach Rechtsgebieten [BAG16]

In Abb. 3-8 erfolgt eine Unterteilung der im Jahr 2013 bei Straßenkontrollen des BAG festgestellten Verstöße zunächst in die Hauptkategorien Fahrpersonalrecht, technische Unterwegskontrolle sowie Ladungssicherung (Kapitel 2.1.3). Wie zuvor bereits erläutert, werden im Bereich Fahrpersonalrecht alle Verstöße im Zusammenhang mit den gesetzlich vorgeschriebenen Lenk-, Ruhe- und Arbeitszeiten erfasst. Alle Vergehen, die in Zusammenhang mit dem technischen Zustand des kontrollierten Fahrzeugs stehen, werden der Kategorie techni- 
sche Unterwegskontrolle zugeordnet. Eine bei der Überprüfung entdeckte nicht oder unzureichend gesicherte Ladung wird der Kategorie Ladungssicherung zugeteilt [BAG16].

\begin{tabular}{|c|c|c|c|}
\hline \multirow{2}{*}{ Verstöße } & \multicolumn{3}{|c|}{ Herkunft des Fahrzeugs } \\
\cline { 2 - 4 } Fahrpersonalrecht & Deutschland & EU15 & $\begin{array}{r}\text { Mittel- und } \\
\text { Osteuropa }\end{array}$ \\
\hline $\begin{array}{c}\text { Kontrollierte Fahrzeuge } \\
\text { Beanstandungsquote }\end{array}$ & 85269 & 28136 & 67654 \\
\hline $\begin{array}{c}\text { Anteil an Beanstandungen } \\
\text { insgesamt }\end{array}$ & $21 \%$ & $21,2 \%$ & $13,2 \%$ \\
\hline $\begin{array}{c}\text { Technische Unterwegskontrolle } \\
\text { Kontrollierte Fahrzeuge }\end{array}$ & $79,2 \%$ & $73 \%$ & $47,8 \%$ \\
\hline $\begin{array}{c}\text { Beanstandungsquote } \\
\text { Anteil an Beanstandungen }\end{array}$ & 25463 & 8349 & 28639 \\
\hline insgesamt & $9,3 \%$ & $11 \%$ & $20,2 \%$ \\
\hline $\begin{array}{c}\text { Ladungssicherung } \\
\text { Kontrollierte Fahrzeuge }\end{array}$ & 28799 & $11,2 \%$ & $30,9 \%$ \\
\hline $\begin{array}{c}\text { Beanstandungsquote } \\
\text { Anteil an Beanstandungen } \\
\text { insgesamt }\end{array}$ & $9 \%$ & $9,5 \%$ & $13 \%, 2 \%$ \\
\hline \begin{tabular}{c} 
Summe Beanstandungen \\
\hline
\end{tabular} & 22636 & 8170 & 18710 \\
\hline
\end{tabular}

Abb. 3-8: In 2013 bei Straßenkontrollen festgestellte Verstöße unterteilt nach Herkunft des Fahrzeugs [ZFF14]

Auf Basis ihrer Zulassung werden Fahrzeuge aus Deutschland, den weiteren Ländern der EU 15 (Mitgliedstaaten bis einschließlich April 2004, siehe Kapitel 7) sowie aus Mittel- und Osteuropa unterschieden. Für die einzelnen Kategorien sind jeweils die Anzahl der kontrollierten Fahrzeuge sowie die Beanstandungsquoten angegeben. Des Weiteren werden bezüglich der jeweiligen Herkunftsbereiche die Anteile an den gesamten Beanstandungen innerhalb einer Kategorie angeführt [ZFF14].

Bei den deutschen Fahrzeugen ist ein Großteil der festgestellten Verstöße, nahezu 80\%, auf Nichteinhaltung der Regeln zum Fahrpersonalrecht zurückzuführen. Mehrheitlich werden dabei Verstöße gegen die Vorgaben zu Lenk- und Ruhezeiten sowie gegen vorgeschriebene Pausen aufgedeckt. Etwa 40\% der Beanstandungen betreffen die Regelungen zum Tachografen, dort werden insbesondere das Fehlen oder die nicht ordnungsgemäße Verwendung von Fahrerkarten bzw. bei älteren Fahrzeugen von Schaublättern sowie das Fehlen von 
Bescheinigungen zu berücksichtigungsfreien Tagen bemängelt. Der Anteil der im Fahrpersonalrecht ermittelten Verstöße an den gesamten Beanstandungen ist bei Fahrern von Fahrzeugen aus den weiteren EU15-Ländern in einem ähnlichen Bereich. Auch die Beanstandungsquoten liegen nahezu gleichauf [ZFF14].

Bei Fahrzeugen aus Mittel- und Osteuropa fallen sowohl der Anteil der Verstöße im Fahrpersonalrecht an den insgesamt festgestellten Verstößen mit unter $50 \%$ als auch die ermittelte Beanstandungsquote für diesen Rechtsbereich mit ca. 13\% deutlich geringer aus. Auffällig ist bei diesen Fahrzeugen jedoch die im Vergleich zu den in Deutschland und den weiteren EU 15 zugelassenen Fahrzeugen hohe Zahl an Verstößen in Bezug auf die technische Fahrzeugsicherheit und Ladungssicherung. Mehr als 30\% der Verstöße betreffen technische Mängel am Fahrzeug, wobei auch die Beanstandungsquote mit ca. 20\% deutlich höher liegt als bei den westeuropäischen Fahrzeugen [ZFF14].

\subsection{Sozialvorschriften und Arbeitsbedingungen}

Die Sozialvorschriften für die gewerbliche Güter- und Personenbeförderung werden auf europäischer Ebene in der Verordnung Nr. 561/2006 sowie darüber hinaus national in der Fahrpersonalverordnung geregelt. Die EU-Verordnung Nr.561/2006 ist in allen EU-Mitgliedsstaaten gültig und betrifft alle Kraftfahrer von Fahrzeugen zur Güterbeförderung, deren zulässiges Gesamtgewicht einschließlich Anhänger 3,5 t überschreitet, sowie alle Kraftfahrer von Fahrzeugen der Personenbeförderung, die für die Beförderung von mehr als 9 Personen einschließlich des Fahrers konstruiert oder dauerhaft angepasst sind. Die Fahrpersonalverordnung weitet die in EU-Verordnung Nr. 561/2006 definierten Vorschriften national auf Fahrten mit Fahrzeugen aus, die zur Güterbeförderung geeignet sind und deren zulässige Gesamtmasse 2,8 t überschreitet. Über die Bestimmungen zu den Lenk- und Ruhezeiten hinaus sind zusätzlich die Vorschriften des Arbeitszeitgesetzes bzw. des Gesetzes zur Regelung der Arbeitszeit von selbstständigen Kraftfahrern zu beachten [BAG14]. Weitergehende Informationen zu den einzelnen Geltungsbereichen sowie zu Ausnahmeregelungen werden in [BAG14] ausgeführt.

In Abb. 3-9 sind die einzelnen Vorgaben der jeweiligen Verordnungen für den gewerblichen Güter- und Personenverkehr detailliert aufgelistet. In der Praxis bedarf es einer ganzheitlichen Betrachtung der Bestimmungen. So sind in der Arbeitszeit neben beispielsweise den Be- und Entladetätigkeiten auch die Lenkzeiten inbegriffen. Bei Ausnutzung der maximalen täglichen Lenkzeit von insgesamt 10 Stunden (zweimal wöchentlich erlaubt) wird gleichzeitig bereits die maximale tägliche Arbeitszeit erreicht. Somit sind neben der Fahrt keine weiteren Tätigkeiten, wie beispielsweise das Entladen des Fahrzeugs, erlaubt. Die maximale zulässige wöchentliche Lenkzeit des Fahrers beträgt 56 Stunden. Jedoch sind für eine Doppelwoche maximal 90 Stunden erlaubt. Somit muss bei der Ausnutzung der wöchentlichen Lenkzeit in der darauffolgenden Woche ein Ausgleich erfolgen, sodass die vorgegebenen 90 Stunden nicht überschritten werden. Darüber hinaus sind auch Tages- und Wochenruhezeiten einzuhalten. Bei einer Tagesruhezeit von mindestens 12 Stunden darf diese auch in eine kleine und eine große Pause aufgeteilt werden, d.h. in eine 3- und eine 9-Stunden 
Pause. Die Vorgaben zur Wochenruhezeit, welche mindestens 45 Stunden beträgt und jede zweite Woche bei entsprechendem Ausgleich auf 24 Stunden reduziert werden kann, greifen nach sechs 24-Stunden-Zeiträumen. Weitergehende Informationen zu den einzelnen Bestimmungen sind [BAG14] zu entnehmen.

\begin{tabular}{|c|c|}
\hline $\begin{array}{l}\text { Arbeitszeit- } \\
\text { bestimmungen } \\
\text { (ArbZG) }\end{array}$ & $\begin{array}{l}\text { > Tägliche Arbeitszeit - Zeit von Beginn bis zum Ende der Arbeit ohne } \\
\text { Ruhepausen: } \\
\text { • Grundsätzlich } 8 \mathrm{~h} \text {; verlängerbar auf } 10 \mathrm{~h} \text { (bei Ausgleich) } \\
\text { • Maximal } 6 \mathrm{~h} \text { ununterbrochene Arbeitszeit } \\
\text { > Ruhepause: } \\
\text { • Mindestens } 30 \mathrm{Min} \text {; bei mehr als } 9 \mathrm{~h} \text { Arbeitszeit mindestens } \\
\quad 45 \text { Min }\end{array}$ \\
\hline $\begin{array}{l}\text { Lenkzeit- } \\
\text { bestimmungen } \\
\text { (EG 561/2006) }\end{array}$ & $\begin{array}{l}\text { > Tägliche Höchstlenkzeit: } \\
\text { • } 9 \text { h; } 2 \text { x wöchentlich } 10 \mathrm{~h} \\
\text { • Wöchentlich: } 56 \mathrm{~h} / \text { Doppelwoche: } 90 \mathrm{~h} \\
\text { • Fahrtunterbrechung: nach spätestens } 4,5 \mathrm{~h} \text { mind. } 45 \text { Min } \\
\quad \Rightarrow \text { Mögliche Aufteilung in } 2 \text { Teile: } 15 \text { Min gefolgt von } 30 \text { Min }\end{array}$ \\
\hline $\begin{array}{l}\text { Ruhezeit- } \\
\text { bestimmungen } \\
\text { (EG 561/2006) }\end{array}$ & $\begin{array}{l}\text { > Tagesruhezeit: } \\
\text { • Mind. } 11 \mathrm{~h} \text { innerhalb von } 24 \mathrm{~h} \text {; } 3 \times \text { wöchentlich auf } 9 \mathrm{~h} \\
\text { verkürzbar (ohne Ausgleich); Aufteilung von mind. } 12 \mathrm{~h} \\
\text { in } 2 \text { Teile ( } 3 \mathrm{~h} \text { gefolgt von } 9 \mathrm{~h} \text { ) } \\
\text { > Wochenruhezeit: } \\
\text { • Nach spätestens } 6 \text { x } 24 \mathrm{~h} \text {-Zeiträumen; Dauer mind. } 45 \mathrm{~h} \text {; } \\
\text { Verkürzung jeder zweiten Wochenruhezeit auf mind. } 24 \mathrm{~h} \\
\text { • Ausgleich bis zum Ende der folgenden dritten Woche } \\
\text { zusammen mit mind. } 9 \mathrm{~h} \text { Ruhezeit }\end{array}$ \\
\hline
\end{tabular}

Abb. 3-9: Gesetzliche Vorgaben zu Lenk-, Ruhe- und Arbeitszeiten [BAG14]

Die Kontrolle der geleisteten Arbeits- und Lenkzeiten erfolgt mithilfe der Fahrerkarte, die jeder Kraftfahrer zum Führen eines Kraftfahrzeugs besitzen muss und die in den digitalen Tachographen eingeführt wird. Bei älteren Fahrzeugen, die vor dem 1. Mai 2006 zugelassen wurden, sind noch mechanische Tachographen verbaut, bei denen die Fahrzeugführer Schaublätter einlegen müssen. Die Fahrerkarten bzw. die Schaublätter zeichnen die Lenk-, Ruhe- und Arbeitszeiten der Fahrzeugführer auf. Bei Unterwegskontrollen durch die BAG oder die Polizei werden die Daten der letzten 28 Kalendertage kontrolliert und gegebenenfalls sanktioniert. Eine Ausnahme bilden Fahrzeuge, die ein zulässiges Gesamtgewicht im Bereich von 2,8 $t$ bis einschließlich 3,5 $t$ besitzen. Hier muss kein Kontrollgerät verbaut sein. Der Fahrzeugführer hat seine Lenk-, Ruhe- und Arbeitszeiten jedoch anderweitig, beispielsweise mittels Protokollen, zu dokumentieren [BAG14].

Die Arbeitsbedingungen von Kraftfahrern sind durch eine Vielzahl unterschiedlicher Belastungen geprägt. Oftmals sind lange und unregelmäßige Arbeitszeiten, hoher Zeit- und Termindruck und eine körperliche Beanspruchung durch langes Sitzen sowie schwere Be- und Entladetätigkeiten an der Tagesordnung [BAS10]. Um eine genaue Charakterisierung dieser 
Einflüsse zu ermöglichen, wurde von der Bundesanstalt für Straßenwesen (BASt) eine Befragungsstudie an Autohöfen sowie auf Betriebsgeländen unter anderem zu den Arbeitsbedingungen, den wesentlichen Belastungsfaktoren, dem Umgang mit tätigkeitsbezogenem Stress sowie dem Fahrverhalten von 555 hauptberuflichen Kraftfahrern durchgeführt, welche Ende 2009 veröffentlicht wurde. Die befragten Fahrer waren im Mittel 43,6 Jahre alt und arbeiteten seit ca. 19 Jahren als Kraftfahrer [EVE10] [EVE11].

In Abb. 3-10 sind auszugsweise zwei Ergebnisse der Befragung abgebildet. Im linken Diagramm wird die Dauer der Abwesenheit von Zuhause dargestellt. Fast die Hälfte der befragten Fahrer ist eine Woche oder länger im Lkw unterwegs, ohne an den Heimatstandort zurückzukehren. Täglich zu Hause sind nur $6 \%$ der Befragten. Wie im rechten Diagramm abzulesen ist, geben mehr als ein Drittel (36\%) der Fahrer an, Probleme bei der ordnungsgemäßen Durchführung der gesetzlich vorgeschriebenen Pausen zu haben. Dies ist sowohl auf nicht ausreichend vorhandene Parkmöglichkeiten als auch auf Zeit- und Terminprobleme zurückzuführen [EVE10] [EVE11].

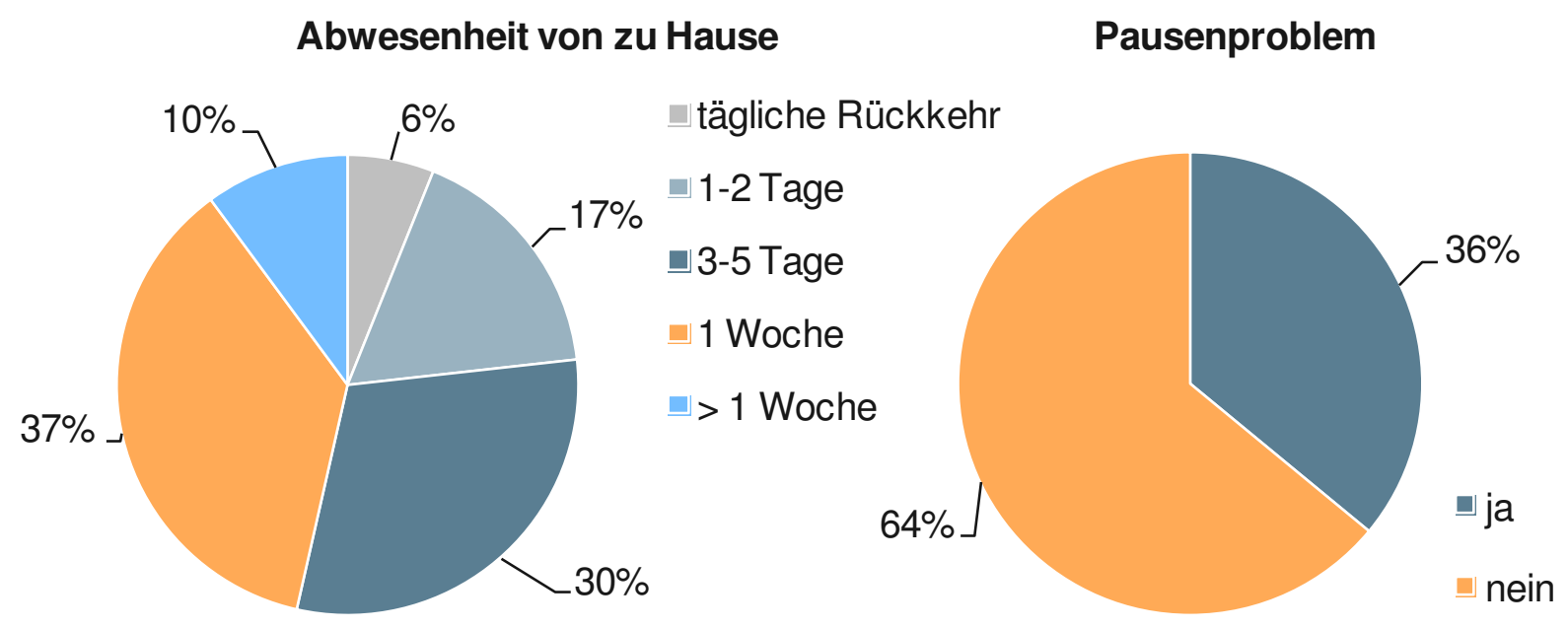

Abb. 3-10: Ergebnisse der Befragung von 555 Lkw-Fahrern auf Autohöfen / Werksgeländen [BAS09]

Die ermittelte durchschnittliche wöchentliche Arbeitszeit beträgt 63,2 Stunden, wobei alleine die reine Lenkzeit mit 46,6 Stunden angegeben wird. Knapp 6\% der Befragten geben als Arbeitszeit sogar mehr als 80 Stunden pro Woche an. Die durchschnittlich zurückgelegte Fahrstrecke beträgt ca. $2800 \mathrm{~km}$ pro Woche. Damit wird in der Studie die sehr hohe Arbeitsbelastung der Kraftfahrer bestätigt. In der subjektiven Wahrnehmung werden die Fahrer am stärksten durch verkehrliche Rahmenbedingungen in ihrer Tätigkeit eingeschränkt [EVE10] [EVE11]. Dabei werden neben den bereits angeführten unzureichenden Park- und Rastmöglichkeiten sowie dem vorherrschenden Termindruck auch riskantes, aggressives oder hinderliches Verhalten anderer Verkehrsteilnehmer, schlechte Straßen und hohe Verkehrsdichte als stärkste Belastungsaspekte genannt [BAS10].

Die detaillierte Auswertung der Umfrageergebnisse ergibt, dass je höher die wöchentliche Arbeitszeit, Fahrzeit und Fahrleistung und je unregelmäßiger die Arbeitszeiten ausfallen, je 
länger die Fahrer nicht nach Hause kommen und je mehr Tätigkeiten die Fahrer zusätzlich zur reinen Fahrtätigkeit ausführen müssen, desto stärker fühlen sie sich körperlich und psychisch belastet. Auf Basis dieser Erkenntnisse werden sechs Fahrertypen identifiziert, die jeweils durch charakteristische Konstellationen des Belastungserlebens, der Stressbewältigung und des Verkehrsverhaltens gekennzeichnet sind. Zwei dieser Fahrertypen, der „Draufgänger-Typ“ mit einem Anteil von ca. 10\% an den befragten Fahrern sowie der "gestresste Typ“ mit einem Anteil von ca. 26\%, werden aufgrund ihres Verhaltens als Risikogruppen für die allgemeine Verkehrssicherheit eingeschätzt [BAS10].

Als Ansatzpunkte zur Erhöhung der Verkehrssicherheit werden in der BASt-Studie Präventivmaßnahmen in verschiedenen Bereichen erarbeitet. Es wird die detaillierte Erläuterung des Themengebiets „Stress und Stressbewältigung“ im Rahmen der gesetzlich vorgeschriebenen Aus- und Weiterbildung empfohlen. Zusätzlich sollten auch weitere für den Transport Mitverantwortliche, beispielsweise Disponenten oder Lageristen, für dieses Themengebiet sensibilisiert werden. Ein weiterer Schwerpunkt wird in der konsequenten Umsetzung der Sozialvorschriften gesehen, was durch umfangreiche und gezielte Straßen- sowie Betriebskontrollen überprüft werden sollte. Weiterhin sind die Schaffung ausreichender Parkmöglichkeiten sowie der Einsatz telematischer Park- und Verkehrsleitsysteme ein möglicher Ansatz zur Reduzierung des Stressniveaus von Kraftfahrern [BAS10].

\subsection{Leicht-Lkw}

Aufgrund des besonderen Fokus auf Leicht-Lkw erfolgt diesbezüglich auch eine separate Analyse des Verkehrsaufkommens. Da generell keine fahrzeugspezifischen Daten zum Verkehrsaufkommen der 12 t-Leicht-Lkw-Kombinationen vorliegen, sind Verkehrszählungen an drei für den Güterverkehr relevanten Knotenpunkten in Westdeutschland durchgeführt und ausgewertet worden.

\subsubsection{Verkehrszählungen}

Bei den im Rahmen der Verkehrszählungen berücksichtigten Verkehrsknotenpunkten handelt es sich um das Aachener Kreuz, für das eine Zählstelle an der A4 auf Höhe der Anschlussstelle Eschweiler West gewählt worden ist, dem Kreuz Köln Ost mit Zählungen auf Höhe der Anschlussstelle Köln-Dellbrück sowie dem Kamener Kreuz, wo der Lkw-Verkehr auf Höhe der Anschlussstelle Bönen erfasst worden ist. Bei den jeweiligen Verkehrszählungen wurden stets beide Fahrtrichtungen betrachtet und verschiedene Uhrzeiten gewählt. Die tatsächliche Zähldauer, d.h. ohne Einbeziehung von Pausen, betrug jeweils vier Stunden. Es wurden dabei zum einen alle 12 t-Leicht-Lkw-Kombinationen und zum Vergleich zusätzlich auch der gesamte relevante Güterkraftverkehr, d.h. alle Fahrzeuge mit einem zGG größer 3,5 t, nach optischem Erscheinungsbild erfasst. Zudem wurden zwei verschiedene Zeiträume berücksichtigt, d.h. vor sowie nach Absenkung der Mautgrenze im Oktober 2015 (Kapitel 2.2). Der erste Zeitraum umfasste dabei Frühjahr und Sommer 2015, während der zweite Zeitraum im Frühjahr 2016 lag. Einen Überblick über die Ergebnisse der an den jeweiligen Zählstellen durchgeführten Verkehrszählungen geben Abb. 3-11, Abb. 3-12 und Abb. 3-13. 


\begin{tabular}{|c|c|c|c|c|}
\cline { 2 - 5 } \multicolumn{1}{c|}{} & \multicolumn{2}{c|}{ A4 - Richtung Köln } & \multicolumn{2}{c|}{ A4 - Richtung Aachen } \\
\hline Datum, Zeit (inkl. Pausen) & Lkw > 3,5 t & $12 \mathrm{t}$ Leicht-Lkw & Lkw > 3,5 t & $12 \mathrm{t}$ Leicht-Lkw \\
\hline Do, 12.03.15, 08:05-12:45 & 1388 & $19(1,36 \%)$ & 1470 & $24(1,63 \%)$ \\
\hline Di, 21.04.15, 11:05-16:15 & 1681 & $23(1,37 \%)$ & 1576 & $27(1,71 \%)$ \\
\hline Do, 14.04.16*, 08:00-13:00 & 1819 & $31(1,7 \%)$ & 1819 & $23(1,26 \%)$ \\
\hline Mi, 04.05.16*, 08:00-13:00 & 1625 & $26(1,6 \%)$ & 1801 & $24(1,33 \%)$ \\
\hline Summe & 6513 & 99 & 6666 & 98 \\
\hline Anteil Leicht-Lkw & \multicolumn{3}{c|}{$1,52 \%$} & \multicolumn{3}{c}{$1,47 \%$} \\
\hline
\end{tabular}

* Nach Einführung der Mautpflicht für Leicht-Lkw

Abb. 3-11: Ergebnisse Zählstelle A4 Höhe Anschlussstelle Eschweiler-West (Kreuz Aachen)

\begin{tabular}{|c|c|c|c|c|}
\cline { 2 - 5 } \multicolumn{1}{c|}{} & \multicolumn{2}{c|}{ A2 - Richtung Dortmund } & \multicolumn{2}{c|}{ A2 - Richtung Hannover } \\
\hline Datum, Zeit (inkl. Pausen) & Lkw > 3,5 t & $12 \mathrm{t}$ Leicht-Lkw & Lkw > 3,5 t & $12 \mathrm{t}$ Leicht-Lkw \\
\hline Mi, 22.04.15, 10:15-16:00 & 2464 & $50(2,03 \%)$ & 2364 & $83(3,51 \%)$ \\
\hline Mi, 29.04.15, 09:45-15:20 & 2380 & $32(1,34 \%)$ & 2758 & $49(1,78 \%)$ \\
\hline Summe & 4844 & 82 & 5122 & 132 \\
\hline Anteil Leicht-Lkw & \multicolumn{2}{|c|}{$1,69 \%$} & \multicolumn{2}{c|}{$2,58 \%$} \\
\hline
\end{tabular}

Abb. 3-12: Ergebnisse Zählstelle A2 Höhe Anschlussstelle Bönen (Kamener Kreuz)

\begin{tabular}{|c|c|c|c|c|}
\cline { 2 - 5 } \multicolumn{1}{c|}{} & \multicolumn{2}{c|}{ A3 - Richtung Leverkusen } & \multicolumn{2}{c|}{ A3 - Richtung Frankfurt } \\
\hline Datum, Zeit (inkl. Pausen) & Lkw > 3,5 t & $12 \mathrm{t}$ Leicht-Lkw & Lkw > 3,5 t & $12 \mathrm{t}$ Leicht-Lkw \\
\hline Fr, 10.07.15, 08:25-13:40 & 2416 & $33(1,37 \%)$ & 1941 & $20(1,03 \%)$ \\
\hline Do, 23.07.15, 08:30-13:30 & 2539 & $26(1,02 \%)$ & 2084 & $28(1,34 \%)$ \\
\hline Mi, 11.05.16*, 09:00-14:05 & 3012 & $80(2,66 \%)$ & 3039 & $62(2,04 \%)$ \\
\hline Summe & 7967 & 139 & 7064 & 110 \\
\hline Anteil Leicht-Lkw & \multicolumn{3}{|c|}{$1,74 \%$} & \multicolumn{3}{c|}{$1,56 \%$} \\
\hline
\end{tabular}

* Nach Einführung der Mautpflicht für Leicht-Lkw

Abb. 3-13: Ergebnisse Zählstelle A3 Höhe Anschlussstelle Köln-Dellbrück (Kreuz Köln-Ost)

Insgesamt zeigen sich infolge der Einführung der Mautpflicht keine signifikanten Unterschiede hinsichtlich der Anteile von 12 t-Leicht-Lkw-Kombinationen. Diese haben eher zuals abgenommen. So fällt der Anteil für die Zählstelle an der A3 bei der nach Einführung der Mautpflicht durchgeführten Zählung etwa doppelt so hoch aus wie bei den beiden Zählungen im Sommer 2015 (Abb. 3-13). 


\subsubsection{Fazit}

Zusammengefasst bewegen sich die Werte der drei Zählstellen zwischen 1,5\% und 2,6\%. Eine seitens der Bundesanstalt für Straßenwesen (BASt) durchgeführte Auswertung der Achslastdaten verschiedener Autobahnmessstellen (dynamische Messung) für den Zeitraum zwischen Januar und Dezember 2014, welche einen Stichprobenumfang von 1.690.961 Lkw mit Anhängern umfasst, ergibt unter Verwendung verschiedener Filterkriterien für $12 \mathrm{t}$-LeichtLkw-Kombination einen Anteil von ca. 2\% $(n=35.738)$, was die Ergebnisse der im Rahmen dieses Projekts durchgeführten Verkehrszählungen bestätigt.

Die angewendeten Leicht-Lkw-Filterkriterien sehen ein zweiachsiges Zugfahrzeug mit einem Gesamtgewicht bis 7,5 t bei einem gleichzeitigen Gewicht des Gesamtzuges bis $12 \mathrm{t}$ vor. Des Weiteren muss der Anhänger ein Zentralachsanhänger mit Einzel- oder Zwillingsachse sein. Zur genaueren Eingrenzung wird zudem ein Achsabstand von mindestens vier Metern zwischen erster und zweiter sowie zwischen zweiter und dritter Achse vorgegeben, d.h. der Abstand der beiden Zugfahrzeugachsen und der Abstand zwischen der Hinterachse des Zugfahrzeugs und der (ersten) Anhängerachse sind Bestandteil der Filterung. 


\section{$4 \quad$ Unfallanalyse}

Die Analyse des Unfallgeschehens in Deutschland von Lkw mit einem zulässigen Gesamtgewicht über 3,5 t basiert auf Daten des Statistischen Bundesamtes (Destatis) für die Jahre 2010 bis 2014 [STA11-15] sowie auf Auswertungen der Unfalldatenbank der Versicherer (UDB). Die Analyse des Lkw-Unfallgeschehens vergleicht die Bundesstatistik und die UDBDaten hinsichtlich Ortslagen, Art der Lkw und Unfallgegner sowie hinsichtlich der jeweiligen Unfallarten, -typen und -folgen. Ferner werden auch Fehlverhalten und der Anteil Hauptverursacher für die beteiligten Lkw-Fahrer betrachtet. Dabei ist stets zu beachten, dass die UDB repräsentativ für das Schadengeschehen der deutschen Versicherer ist und sich die Datenbasis somit grundlegend von der Bundesstatistik unterscheidet. Neben den Vergleichen zur Bundesstatistik werden mittels der UDB-Daten noch weiterführende Auswertungen durchgeführt, die mittels der öffentlich verfügbaren Destatis-Daten nicht möglich sind.

Ein weiterer Fokus der Unfallanalyse liegt auf 12 t-Leicht-Lkw-Kombinationen. Zur näheren Charakterisierung des Unfallgeschehens dieser leichten Gliederzüge werden im Zuge einer Sonderabfrage beim Statistischen Bundesamt zunächst entsprechende fahrzeugspezifische Auswertungen der Bundesstatistik analysiert. Des Weiteren erfolgen der Aufbau und die Auswertung einer Unfalldatenbank mit 150 recherchierten und auf Seitenwind zurückzuführenden Leicht-Lkw-Unfällen sowie eine Analyse von Schadensakten eines Versicherers.

\subsection{Unfälle von Güterkraftfahrzeugen über 3,5 t zGG im Straßenverkehr}

Im Folgenden werden für Lkw mit einem zGG größer 3,5 t zunächst sowohl die Bundesstatistik als auch die UDB hinsichtlich verschiedener Unfallmerkmale ausgewertet und verglichen.

\subsubsection{Bundesstatistik im Vergleich zur Unfalldatenbank der Versicherer}

Die in der UDB dokumentierten Fälle basieren auf Schadenakten der Kraftfahrt-Haftpflichtversicherer und setzen für die Erfassung stets Personenschaden und einen Gesamtschadenaufwand von mindestens $15.000 €$ voraus, sodass bei der Auswertung der Bundesstatistik ebenfalls nur Unfälle mit Personenschaden herangezogen werden. Während die UDBDaten 834 Lkw-Unfälle mit insgesamt 974 beteiligten Fahrern von Güterkraftfahrzeugen über 3,5 t zGG im Zeitraum von 2000 bis 2011 umfassen, liegt die entsprechende Anzahl in den Destatis-Daten für Unfälle mit Personenschaden im Zeitraum von 2010 bis 2014 bei 88.239.

\subsubsection{Ortslage}

Bei der Ortslage eines Unfalls wird zunächst zwischen innerorts und außerorts unterschieden. Für Letzteres erfolgt zudem eine Untergliederung in Unfälle, die auf Autobahnen stattgefunden haben. Die jeweiligen Anteile für die an Unfällen mit Personenschaden beteiligten Fahrer von Güterkraftfahrzeugen mit einem zGG größer 3,5 t innerhalb der Destatis- und UDB-Daten gehen aus Abb. 4-1 hervor. Dabei zeigt die linke Säule die Ergebnisse der Destatis und die rechte Säule die Ergebnisse der UDB-Auswertung. Dies gilt analog auch für die nachfolgenden Diagramme. In der Bundesstatistik sind ca. ein Drittel der Fahrer an 
einem Unfall auf der Autobahn beteiligt. Dieser Anteil ist für die UDB mit 43\% nochmals erheblich höher. Dafür fällt hier der Anteil für innerorts stattgefundene Unfälle gegenüber den Destatis-Daten deutlich geringer aus, wo sie mit fast $40 \%$ die höchste Relevanz haben.

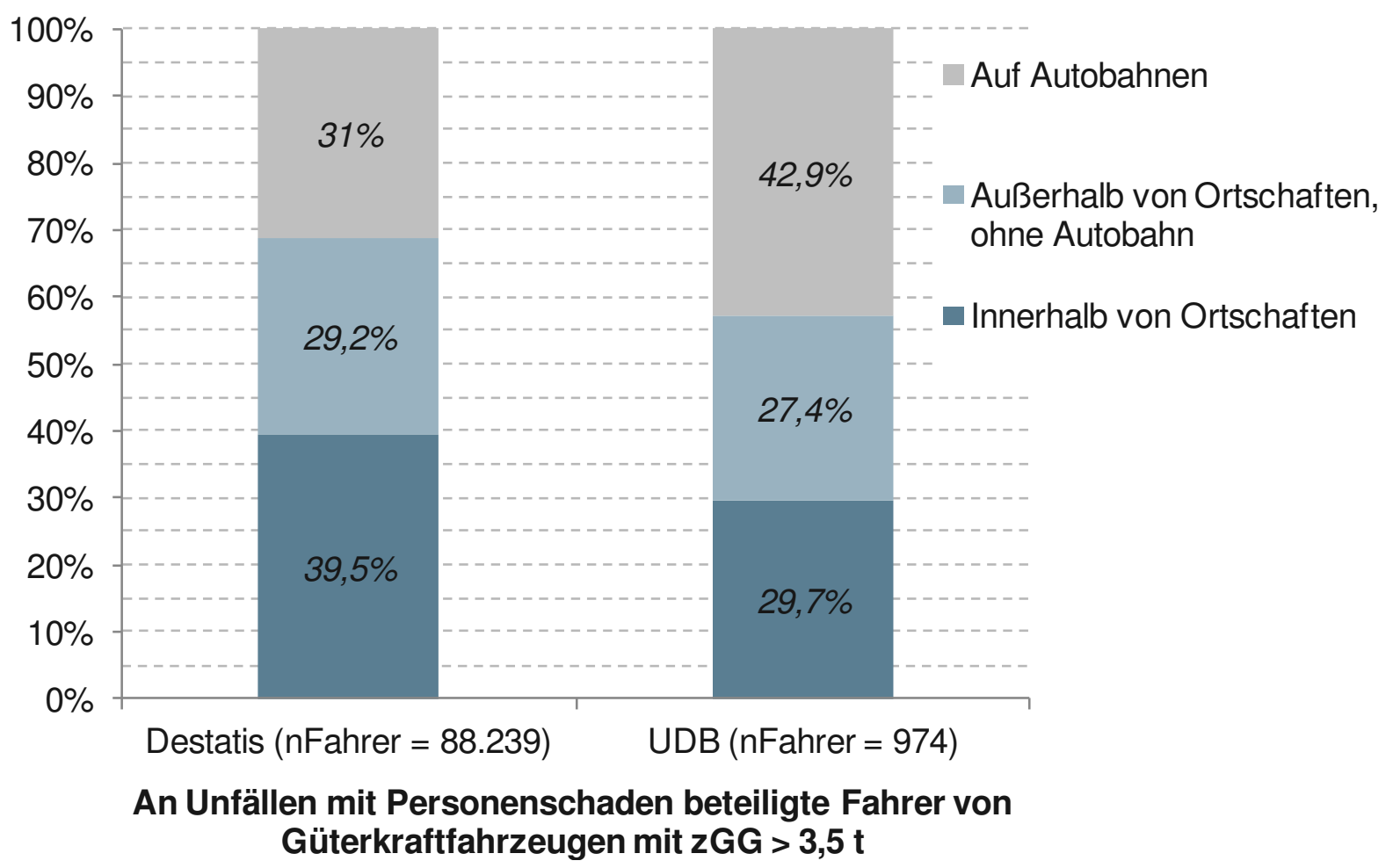

Abb. 4-1: Vergleich Destatis (2010-2014) [STA11-15] \& UDB nach Ortslage

Betrachtet man in beiden Datensätzen nur Unfälle mit Getöteten, so weist bei Destatis die Ortslage „Außerhalb von Ortschaften, ohne Autobahn“ mit 40\% den größten Anteil auf, während in der UDB mit 46\% die Autobahn weiterhin dominierend ist (Anhang, Abb. 9-1). Demgegenüber sind nur jeweils ca. $20 \%$ der Fahrer an tödlichen Unfällen innerorts beteiligt.

Gliedert man die Güterkraftfahrzeuge der an Unfällen mit Personenschaden beteiligten Fahrer nach N2- und N3-Fahrzeugen sowie Sattelzugmaschinen (Abb. 9-2 bis Abb. 9-4), so zeigt sich für N2-Fahrzeuge innerorts die höchste Relevanz (Destatis: 55\%, UDB: 44\%) während für Sattelzugmaschinen Autobahnunfälle am häufigsten sind (Destatis: 47\%, UDB: 53\%).

\subsubsection{Fahrzeugart}

Im Rahmen der Unfallanalyse werden die Fahrzeugklassen N2 und N3 bei der Auswertung nach Fahrzeugart nochmals in verschiedene Gewichtsklassen sowie in Lkw ohne Angaben zum zGG unterteilt. Sattelzugmaschinen und Lkw mit Spezialaufbau werden dabei separat berücksichtigt. Bezüglich der Destatis-Daten ist zu erwähnen, dass hier die Aufgliederung der Lkw nach Gewichtsklassen nur für deutsche Fahrzeuge erfolgt während ausländische Fahrzeuge als „Lkw ohne Angabe zum zGG“ geführt werden. Dies gilt nicht für die separat betrachteten Fahrzeugarten Sattelzugmaschine und Lkw mit Spezialaufbau. Hier sind ausländische Fahrzeuge stets mit enthalten. Des Weiteren umfassen die „Lkw ohne Angabe 
zum zGG“ in den Destatis-Auswertungen bis 2013 auch N1-Fahrzeuge, sodass zwangsläufig ein geringer Anteil an N1-Fahrzeugen in den Daten verbleibt. Für das Jahr 2014 ist dieser auf Basis einer Sonderabfrage separiert worden, welche erforderlich war, da seit $2014 \mathrm{Ge}$ wichtsklassen in der allgemeinen amtlichen Unfallstatistik nicht mehr gesondert ausgewiesen werden. Die unter den genannten Randbedingungen für die Bundesstatistik und die UDB jeweils ausgewerteten Anteile der verschiedenen Fahrzeugarten gehen aus Abb. 4-2 hervor.

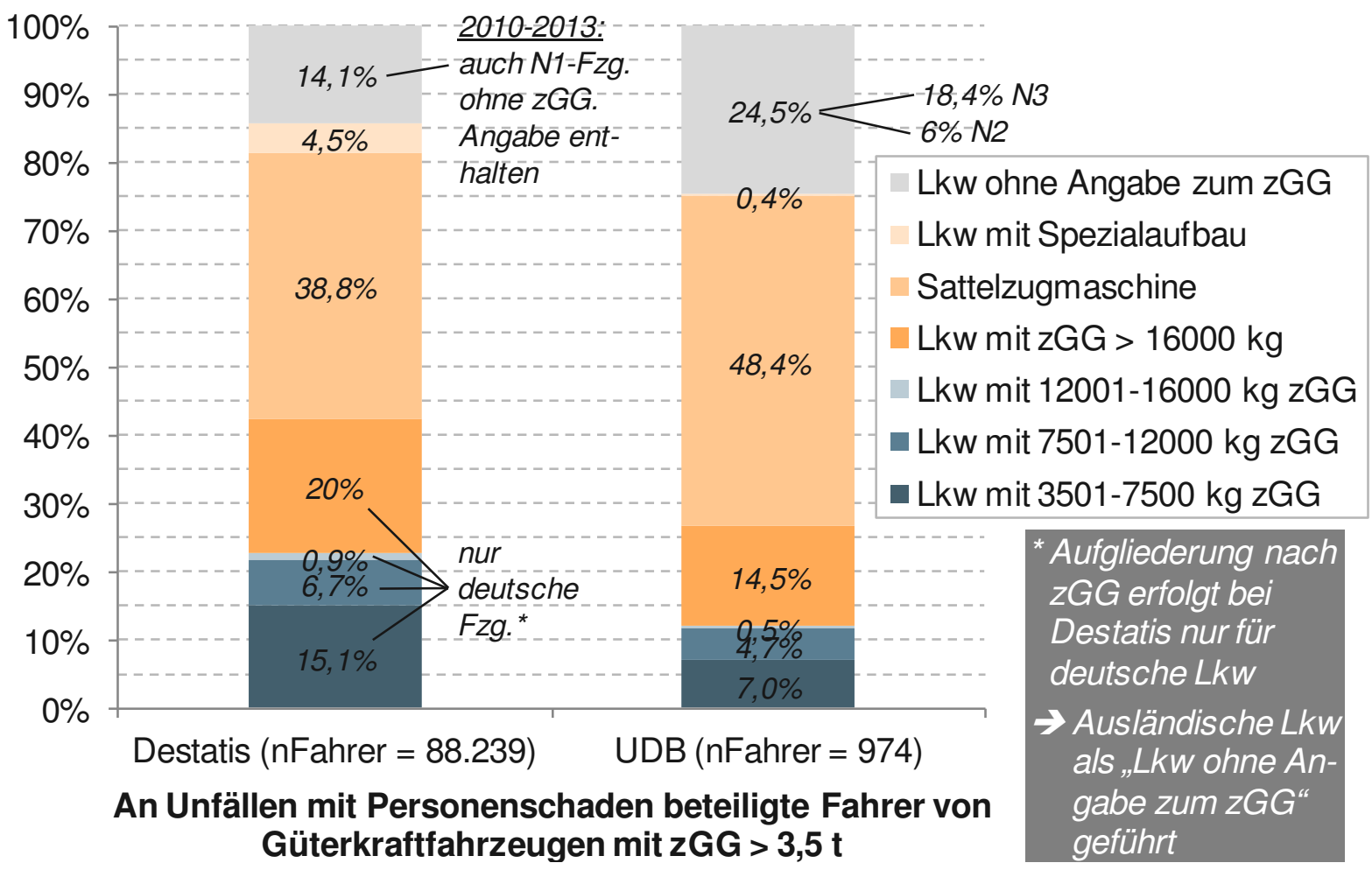

Abb. 4-2: Vergleich Destatis (2010-2014) [STA11-15] \& UDB nach Fahrzeugart

Es zeigt sich, dass Sattelzugmaschinen die größte Fahrzeuggruppe bilden, wobei deren Anteil in der UDB nochmals höher ausfällt. Daneben kommen N3-Fahrzeuge (Lkw > $12 \mathrm{t}$ ) auf einen Anteil von $21 \%$ in den Destatis und $15 \%$ in den UDB-Daten. Letzterer erhöht sich auf $33 \%$, sofern man die als N3 klassifizierten Fahrzeuge aus der Kategorie „Lkw ohne Angabe zum zGG" mit einrechnet. Für N2-Fahrzeuge (Lkw > 3,5 $\mathrm{t} \& \leq 12 \mathrm{t}$ ) betragen die entsprechenden Anteile 22\% und 12 bzw. 18\%. Der geringe Anteil von Lkw mit Spezialaufbau in der UDB ist darauf zurückzuführen, dass diese dort häufig als normale Lkw klassifiziert werden.

Bei Beschränkung der Auswertung auf Unfälle mit Getöteten zeigt sich eine Zunahme der Anteile für schwere Güterkraftfahrzeuge (Abb. 4-3). So steigt der Anteil der an tödlichen Unfällen beteiligten Sattelzugmaschinen in den Destatis-Daten von 39\% auf über 48\%, während der Anteil für Lkw mit einem zGG größer $16 \mathrm{t}$ indes nur leicht zunimmt. Bei den UDB-Daten zeigt sich ein etwas anderes Bild. Hier nimmt der Anteil der Sattelzugmaschinen nur leicht zu (von 48\% auf 51\%), wohingegen bei den Lkw mit einem zGG größer 16 t eine Zunahme von $14,5 \%$ auf $20 \%$ erfolgt. Hier nimmt zudem auch der Anteil für leichte Lkw mit einem zGG zwischen 3,5 und 7,5 t zu (von 7\% auf 9\%), was bei den Destatis-Daten nicht der Fall ist. 


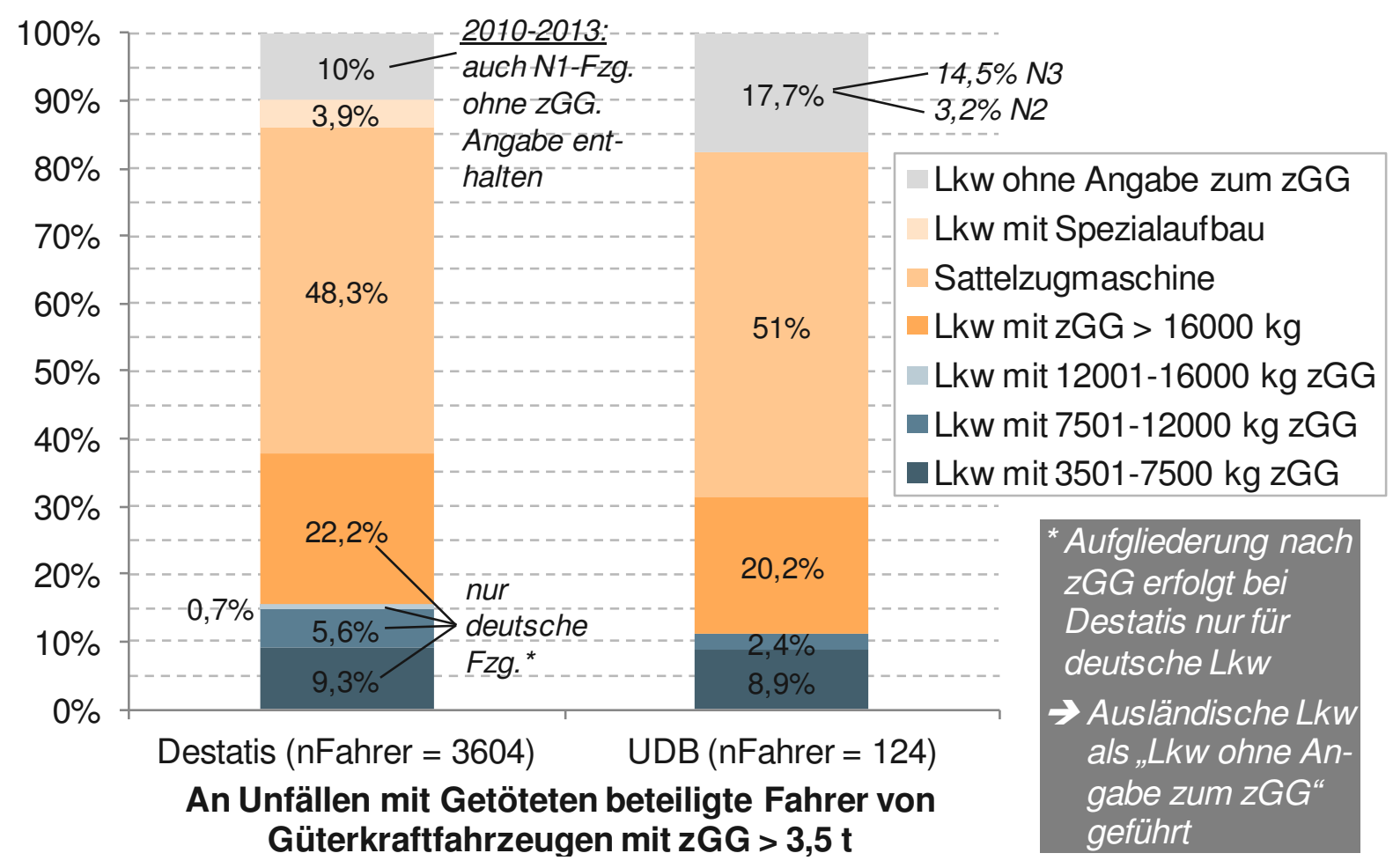

Abb. 4-3: Vergleich Destatis (2010-2014) [STA11-15] \& UDB bezüglich Unfällen mit Getöteten nach Fahrzeugart

Anhand der Destatis-Daten kann für den betrachteten Zeitraum von 2010 bis 2014 durch Bezug der Zahl der Unfälle mit Personenschaden auf den durchschnittlichen deutschen Fahrzeugbestand der jeweiligen Fahrzeugart das entsprechende Unfallrisiko berechnet werden, d.h. die Zahl der im Durchschnitt pro 1000 zugelassene Fahrzeuge an Unfällen beteiligten deutschen Güterkraftfahrzeuge (Abb. 4-4).

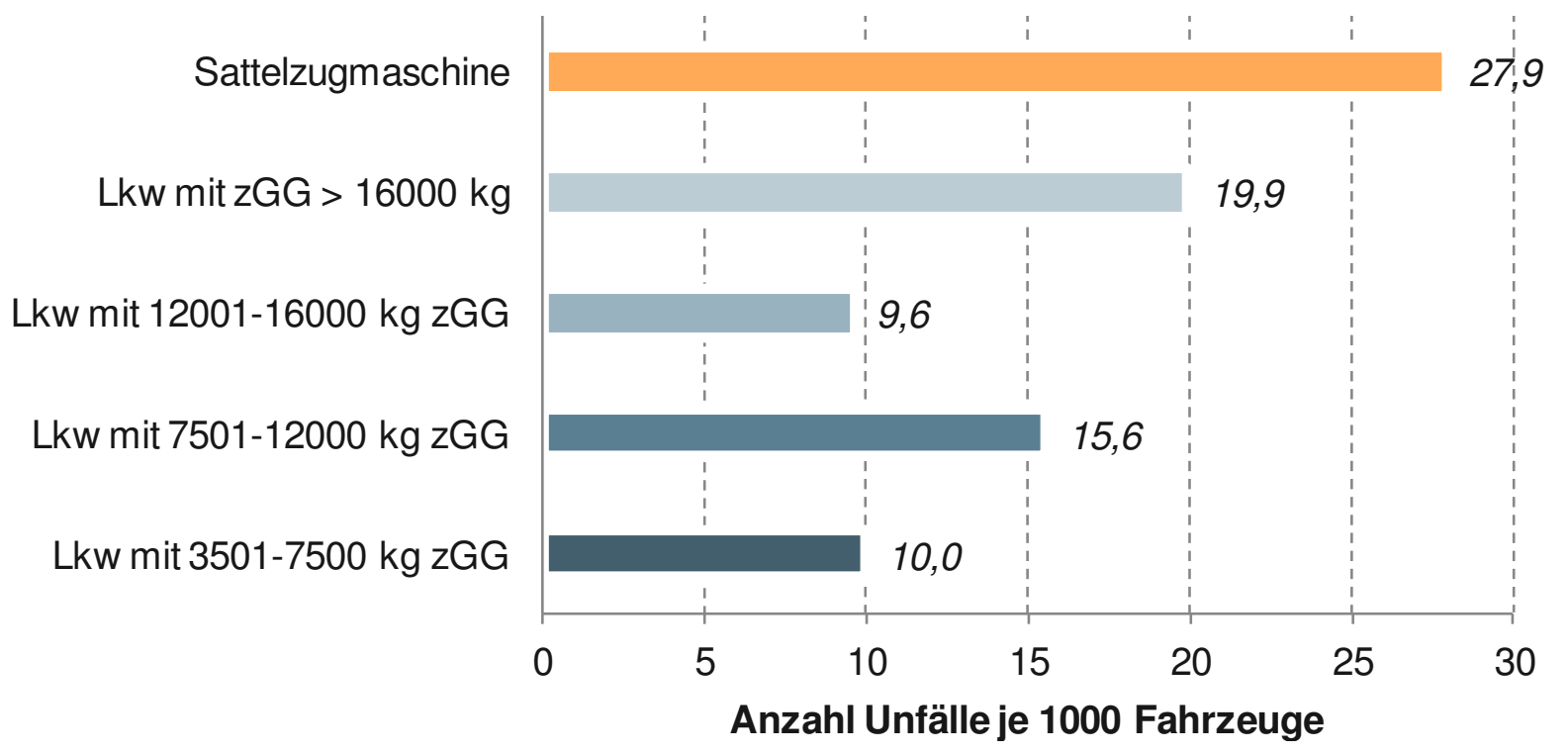

Abb. 4-4: An Unfällen mit Personenschaden beteiligte Fahrer pro 1000 Fzg. (Destatis, 2010-2014, Bezug auf Gesamtbestand deutscher Fzg. gemäß KBA) [STA11-15] 
Da die Aufgliederung der Lkw nach Gewichtsklassen in den Destatis-Daten ohnehin nur für deutsche Fahrzeuge erfolgt und die entsprechenden Bezugsdaten des Kraftfahrt-Bundesamtes (KBA) zum Bestand ebenfalls nur deutsche Fahrzeuge berücksichtigen, beziehen sich die Werte in Abb. 4-4 allein auf deutsche Fahrzeuge. Dabei ist das allgemeine Unfallrisiko für Sattelzugmaschinen bzw. Sattelzüge am höchsten, gefolgt von schweren Lkw (Solofahrzeuge \& Gliederzüge) mit einem zGG größer 16 t. Bei Sattelzugmaschinen sind bezogen auf 1000 Fahrzeuge durchschnittlich 28 in Unfälle mit Personenschaden verwickelt, im Falle von schweren Lkw sind es 20. Nimmt man Unfälle mit schwerwiegendem Sachschaden (genaue Definition in Kapitel 4.2.1.1) hinzu, so kommt man auf eine Zahl von 41 Sattelzugmaschinen und 28 schweren Lkw. Der Anteil von Unfällen mit Beteiligung ausländischer Fahrzeugen beträgt für Sattelzugmaschinen laut Auskunft des Statistischen Bundesamtes 26,6\% und ist nicht mit eingerechnet. Des Weiteren werden seit 2012 Lkw mit Spezialaufbau hinsichtlich des Bestandes nicht gesondert betrachtet, sondern ebenfalls dem zGG nach eingeordnet, was für die Jahre 2010 und 2011 eine entsprechende Umrechnung erforderlich macht.

\subsubsection{Hauptverursacher}

Die Anteile in den Destatis und UDB-Daten, in der ein Fahrer der jeweiligen Fahrzeugart Hauptverursacher eines Unfalls mit Personenschaden ist, gehen aus Abb. 4-5 hervor.

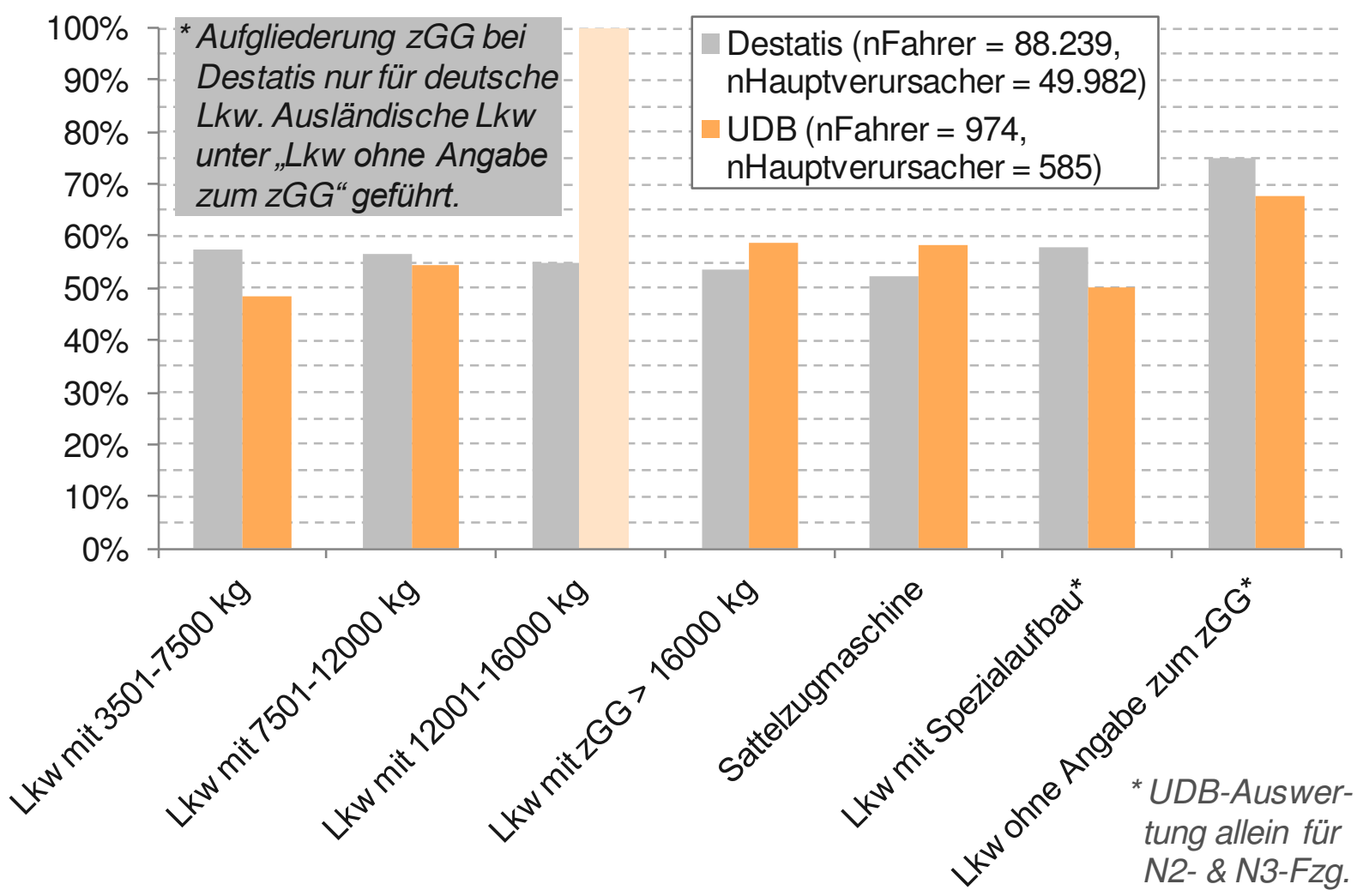

Anteil Hauptverursacher der an Unfällen mit Personenschaden beteiligten Fahrer von Güterkraftfahrzeugen mit zGG größer 3,5 t nach Fahrzeugart

Abb. 4-5: Vergleich Destatis (2010-2014) [STA11-15] \& UDB hinsichtlich Anteil Hauptverursacher nach Fahrzeugart in Unfällen mit Personenschaden 
Wie in Kapitel 4.1.1.2 erläutert, erfolgt die Aufgliederung nach zGG bei Destatis allein für deutsche Fahrzeuge. Ausländische Lkw fallen in die Kategorie „Lkw ohne Angabe zum zGG“. Nur bei den Fahrzeugarten Sattelzugmaschine und Lkw mit Spezialaufbau sind ausländische Fahrzeuge inbegriffen. Generell sind die beteiligten Fahrer aller betrachteten Fahrzeugarten in etwa der Hälfte der Unfälle mit Personenschaden auch Hauptverursacher. In den Destatis-Daten liegen die Anteile zwischen 52\% und 58\%, wobei sie bei den Lkw mit Angabe des zGG zu den leichteren Fahrzeugkategorien hin ansteigen. Nur für die Gruppe der Lkw ohne Angabe zum zGG liegt ein erhöhter Anteil von 75\% vor. Dies gilt auch für die UDBDaten, wo 68\% der Fahrer von Lkw ohne Angabe zum zGG Hauptverursacher des Unfalls sind, wobei hier ausschließlich N2 und N3-Fahrzeuge Teil der Auswertung sind. Ansonsten reichen die Anteile von $49 \%$ bis 59\% und liegen damit in einem ähnlichen Wertebereich wie in den Destatis-Daten. Im Gegensatz dazu zeigt sich hier allerdings ein Anstieg der Anteile hin zu den schweren Güterkraftfahrzeugen. So sind Fahrer von Lkw mit einem zGG größer 16 t zu 59\% und Fahrer von Sattelzugmaschinen zu 58\% Hauptverursacher des Unfalls.

Der sich in den UDB-Daten für Fahrer von Lkw mit einem zGG zwischen $12 \mathrm{t}$ und $16 \mathrm{t}$ ergebene Anteil von $100 \%$ ist statistisch nicht von Relevanz, da dieser insgesamt nur auf fünf Fahrern basiert. Der Grund hierfür liegt in der vorgegebenen Obergrenze von $16 \mathrm{t}$, welche sich an der entsprechenden Definition des Statistischen Bundesamtes orientiert, aber nicht mehr den tatsächlichen Gewichtsklassen aktueller Fahrzeuge entspricht. Vielmehr sollte die Obergrenze bei $18 \mathrm{t}$ liegen. Diese erweiterte Kategorie würde im Rahmen der UDB-Auswertung 35 Fahrer umfassen und wäre damit deutlich aussagekräftiger. Hierbei läge der Anteil der Hauptverursacher bei $69 \%$, was allerdings immer noch deutlich höher wäre als für die anderen Fahrzeugarten.

\subsubsection{Unfallfolgen}

Betrachtet man die Verteilung der an Unfällen mit Personenschaden beteiligten Güterkraftfahrzeuge mit einem zGG größer 3,5 t hinsichtlich Unfällen mit Getöteten, Schwerverletzten und Leichtverletzten, so ergeben sich deutliche Unterschiede zwischen den Destatis und UDB-Daten (Abb. 4-6). Die Anteile der Fahrer, welche in Unfälle mit Getöteten und Schwerverletzten verwickelt sind, fallen für die UDB wesentlich höher aus. Während in den DestatisDaten der Anteil für Unfälle mit Getöteten oder Schwerverletzten knapp 28\% beträgt, liegt dieser in der UDB bei 54\%.

Die in Abb. 4-6 dargestellte Aufteilung in Unfälle mit Getöteten, Schwerverletzten und Leichtverletzten erfolgt in Abb. 9-5, Abb. 9-6 und Abb. 9-7 im Anhang nochmals separat für N2und N3-Fahrzeuge sowie Sattelzugmaschinen. Dabei zeigt sich für N3-Fahrzeuge und Sattelzugmaschinen ein etwas höherer Anteil von Unfällen mit Getöteten als für N2-Fahrzeuge. Dies gilt auch in Bezug auf Schwerverletzte, wobei in den UDB-Daten die Unterschiede deutlich größer ausfallen. So beträgt hier für N2-Fahrzeuge der Anteil von Unfällen mit Schwerverletzten 33\%, was deutlich unter den Anteilen für N3-Fahrzeuge und Sattelzugmaschinen liegt, welche $45 \%$ und $41 \%$ betragen. Somit sind die Unfallfolgen bei Beteiligung schwerer Nutzfahrzeuge häufiger schwerwiegend. Das zeigt sich auch in der Bundesstatistik. 


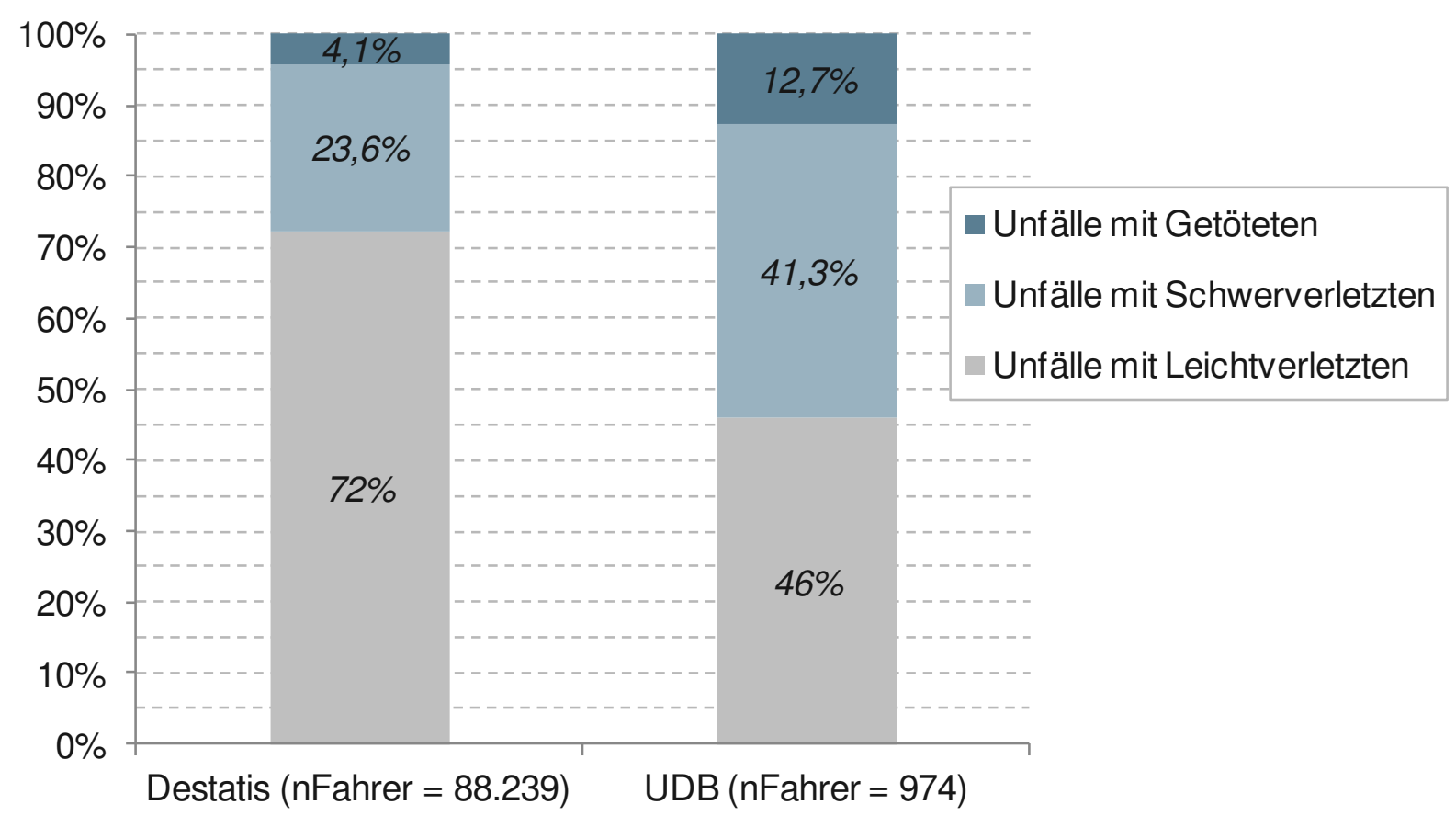

An Unfällen mit Personenschaden beteiligte Fahrer von Güterkraftfahrzeugen mit zGG > 3,5 t

Abb. 4-6: Vergleich Destatis (2010-2014) [STA11-15] \& UDB nach Unfallfolgen

\subsubsection{Unfallart}

Ein Vergleich der Verteilung der Unfallarten ergibt für beide Datenbanken eine Übereinstimmung hinsichtlich der Rangfolge der wichtigsten Szenarien (Abb. 4-7). So bildet der Zusammenstoß mit einem vorausfahrenden/wartenden Fahrzeug die häufigste Unfallart (Destatis: 27\%, UDB: 34\%), gefolgt vom Zusammenstoß mit einem einbiegenden / kreuzenden Fahrzeug (Destatis: 17\%, UDB: 19\%) sowie jeweils dem Zusammenstoß mit einem seitlich in gleicher Richtung fahrenden Fahrzeug (Destatis: 14\%, UDB: 16\%) und einem entgegenkommenden Fahrzeug (Destatis: 12\%, UDB: 13\%).

Während das Abkommen von der Fahrbahn nach rechts oder links in den Destatis-Auswertungen zusammen einen Anteil von 10\% aufweist, sind diese Unfallarten in der UDB unterrepräsentiert und kommen auf einen Anteil von unter 4\%. Dahingegen sind die Anteile für Zusammenstöße mit einem anfahrenden/anhaltenden/ruhenden Fahrzeug und Zusammenstöße zwischen Fahrzeug und Fußgänger wieder vergleichbar.

Beschränkt man die Auswertung nach Unfallart auf Güterkraftfahrzeuge, die an Unfällen mit Getöteten beteiligt sind, so zeigt sich sowohl für die Destatis als auch für die UDB-Daten eine deutliche Erhöhung der Anteile für Zusammenstöße mit einem entgegenkommenden Fahrzeug sowie für Zusammenstöße zwischen Fahrzeug und Fußgänger (Anhang, Abb. 9-8). Diese fallen jeweils für beide Unfallarten mehr als doppelt so hoch aus wie in Abb. 4-7. Der Anteil für einen Zusammenstoß mit einem entgegenkommenden Fahrzeug steigt in der Destatis-Auswertung von $12 \%$ auf $29 \%$ und für die UDB von $13 \%$ auf $27 \%$. Be- 
züglich eines Zusammenstoßes zwischen Fahrzeug und Fußgänger kommt es bei Destatis sogar zu einer Verdreifachung des Anteils auf $9 \%$ während der Anteil für die UDB von 4\% ebenfalls auf $9 \%$ ansteigt. Abnahmen der Anteile gibt es vor allem für Zusammenstöße mit seitlich in gleicher Richtung fahrenden sowie einbiegenden / kreuzenden Fahrzeugen.

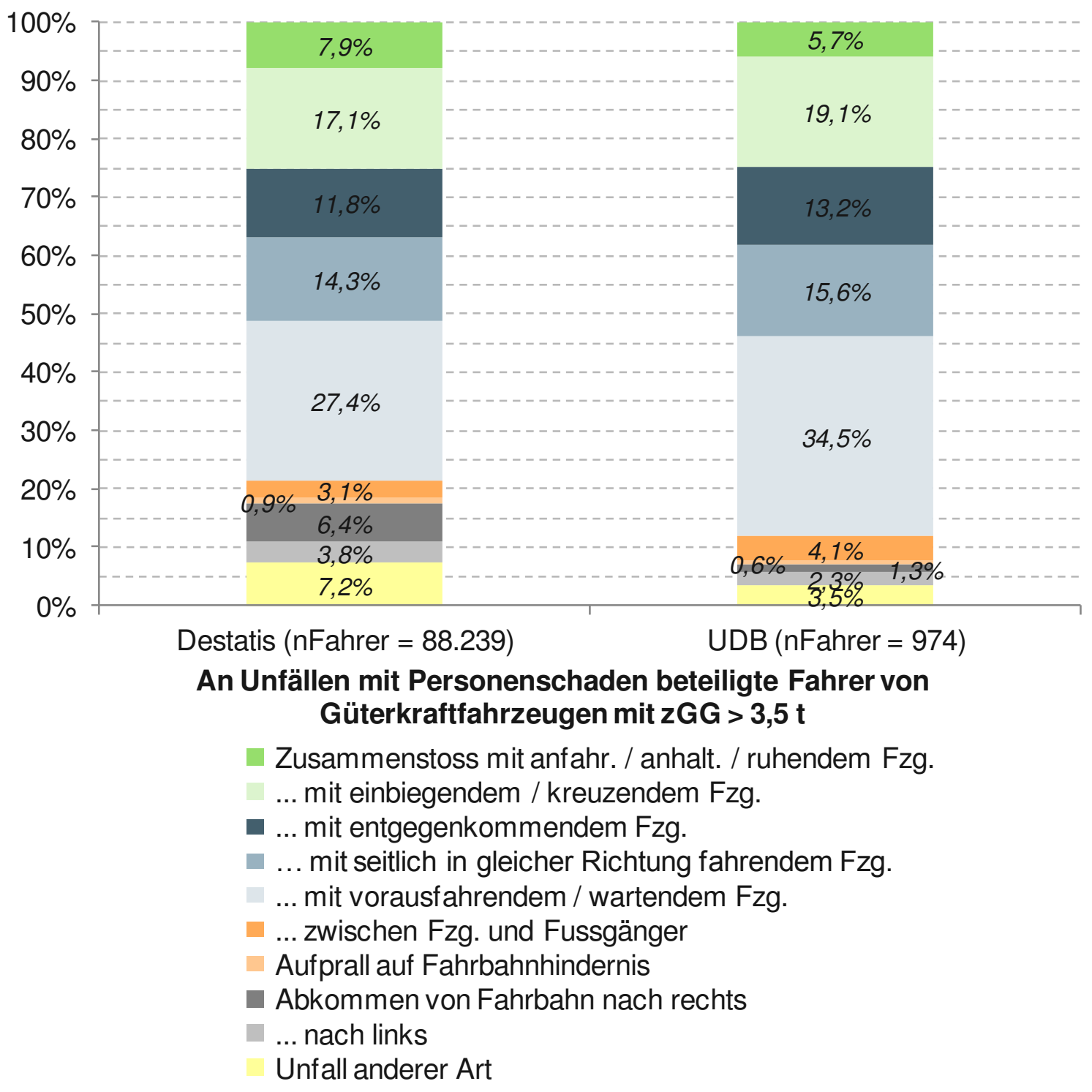

Abb. 4-7: Vergleich Destatis (2010-2014) [STA11-15] \& UDB nach Unfallart

Die UDB-Daten ermöglichen noch weiterführende Auswertungen. So werden die untersuchten Unfallarten in Abb. 4-8 um Zusammenstöße zwischen Fahrzeug und Radfahrer sowie Zusammenstöße zwischen Fahrzeug und motorisiertem Zweirad ergänzt, welche im Rahmen der Destatis-Definition als Fahrzeuge betrachtet und somit im Gegensatz zu Fußgängern nicht separat aufgeführt werden. Es wird ersichtlich, dass die Anteile von Zusammenstößen mit Radfahrern und motorisierten Zweirädern mit jeweils 6\% sogar höher ausfallen als der entsprechende Fußgängeranteil. Zuvor wurden die meisten Radfahrerunfälle der Unfallart „Zusammenstoß mit einbiegendem / kreuzendem Fahrzeug“ zugeordnet, was auch für Unfälle mit motorisierten Zweirädern gilt, welche sich im Vergleich zu den Radfahrerunfällen 
aber insgesamt deutlich breiter auf die anderen Unfallarten verteilten. Hier sind vor allem auch Zusammenstöße mit entgegenkommenden Fahrzeugen und Zusammenstöße mit seitlich in gleicher Richtung fahrenden Fahrzeugen zu nennen. Beschränkt man auch hier die Auswertung auf Unfälle mit Getöteten, so erhöht sich der Anteil für Zusammenstöße mit Radfahrern auf $8 \%$ und der Anteil für Zusammenstöße mit motorisierten Zweirädern auf $10 \%$.

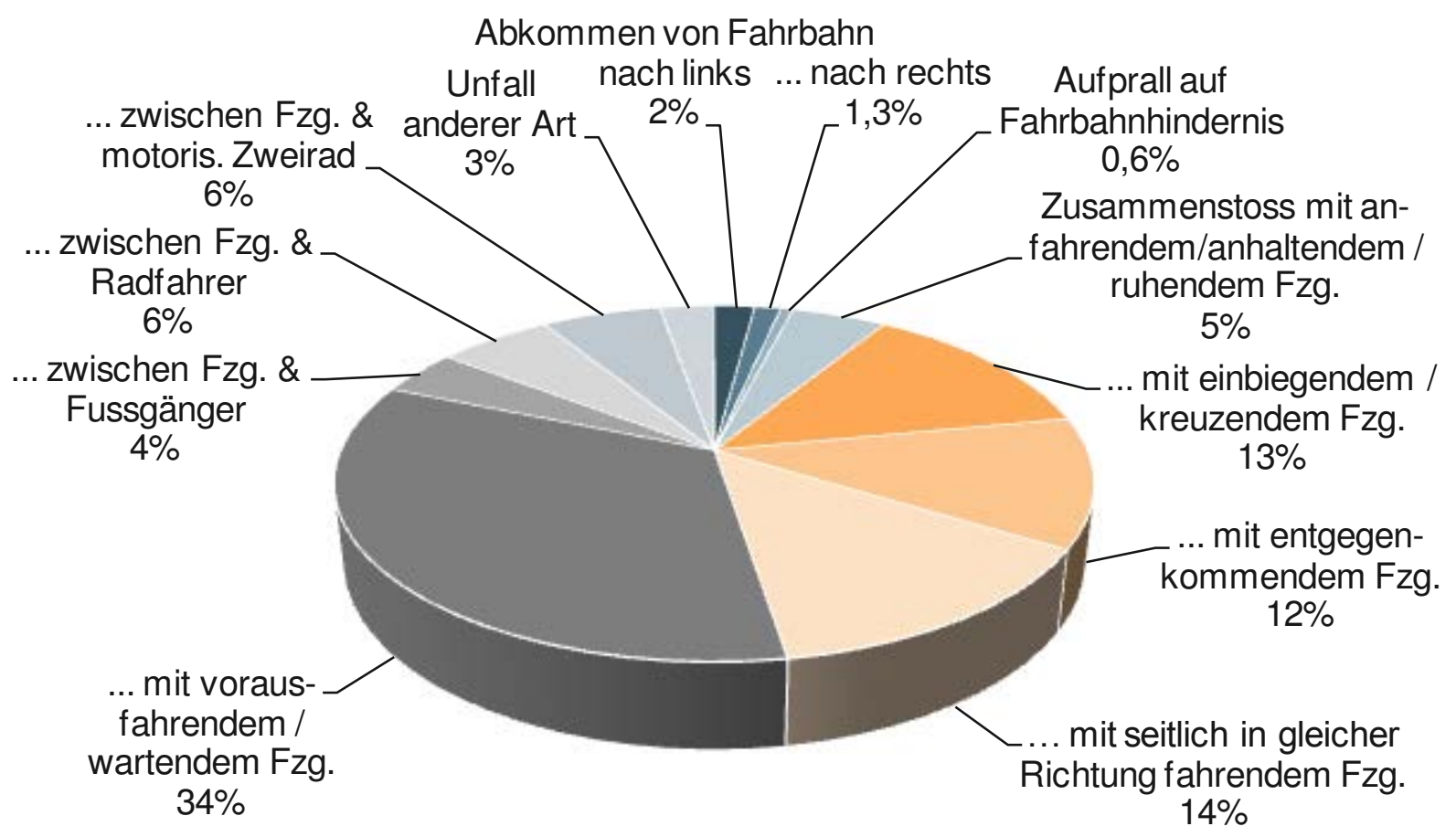

Abb. 4-8: An Unfällen beteiligte Fahrer von Güterkraftfahrzeugen mit zGG größer 3,5 t nach Unfallart (UDB, $\mathrm{n}=974$ )

Gliedert man die in Abb. 4-8 dargestellte Auswertung der UDB nach Unfallart nochmals hinsichtlich N2 und N3-Fahrzeugen sowie Sattelzugmaschinen, so ergeben sich fahrzeugspezifische Unterschiede. Bei N2-Fahrzeugen nimmt der Anteil für Zusammenstöße mit einbiegenden / kreuzenden Fahrzeugen auf $18 \%$ zu, während sich der Anteil für Zusammenstöße mit seitlich in gleicher Richtung fahrenden Fahrzeugen halbiert (Anhang, Abb. 9-9). In Bezug auf N3-Fahrzeuge zeigt sich eine leichte Erhöhung der Anteile von Unfällen mit ungeschützten Verkehrsteilnehmern und wiederum eine deutliche Reduzierung des Anteils für Zusammenstöße mit seitlich in gleicher Richtung fahrenden Fahrzeugen (9\%), wie aus Abb. 9-10 im Anhang hervorgeht. Entsprechend hoch ist für Sattelzugmaschinen die Relevanz von Zusammenstößen mit seitlich in gleicher Richtung fahrenden Fahrzeugen (Anhang, Abb. 9-11). Diese Unfallart trifft hier auf 19\% der beteiligten Fahrzeuge zu. Im Gegensatz zu N3-Fahrzeugen nehmen für Sattelzugmaschinen die Anteile von Unfällen mit ungeschützten Verkehrsteilnehmern etwas ab.

\subsubsection{Unfalltyp}

Sowohl für die Destatis-Daten als auch für die UDB stellt der Unfall im Längsverkehr den mit Abstand häufigsten Unfalltypen dar. Dieser trifft jeweils auf ca. die Hälfte der an Unfällen mit 
Personenschaden beteiligten Fahrer zu (Abb. 4-9). Generell zeigt sich eine gute Übereinstimmung zwischen Destatis und UDB hinsichtlich der Verteilung der Unfalltypen.

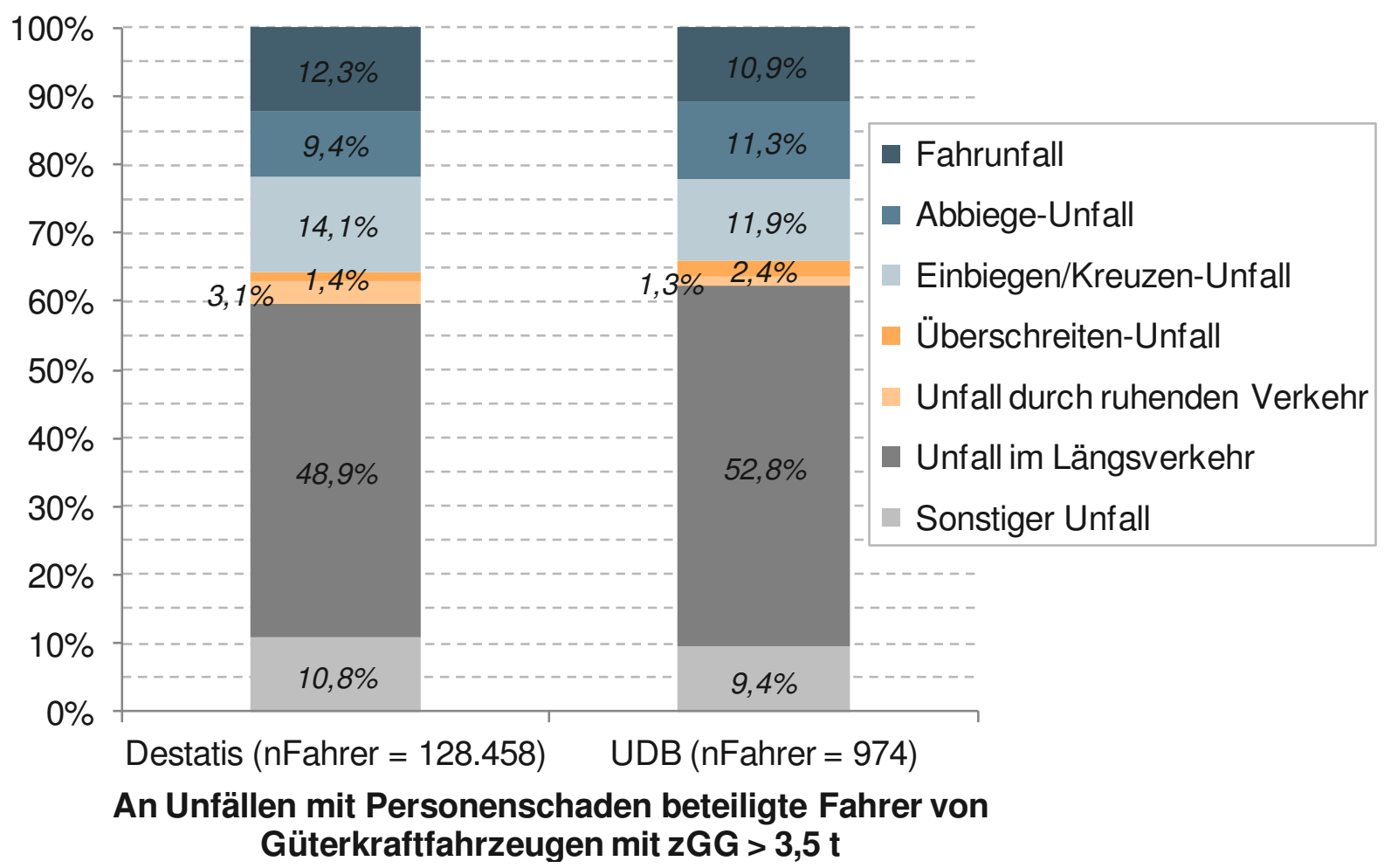

Abb. 4-9: Vergleich Destatis (2010-2014) [STA11-15] \& UDB nach Unfalltyp

Bei Beschränkung der Auswertung auf Unfälle mit Getöteten nimmt die Relevanz von Unfällen im Längsverkehr in den Destatis-Daten mit 50\% nochmals leicht zu, während sie für die UDB mit 46\% etwas abnimmt (Anhang, Abb. 9-12). Für beide Datensätze ist ein Anstieg bei den Fahr- und Überschreitenunfällen zu verzeichnen. Letzterer Unfalltyp bezieht sich definitionsgemäß allein auf Unfälle mit Fußgängern.

Betrachtet man in den UDB-Daten ausschließlich N2-Fahrzeuge, so nehmen die Anteile für Fahr- sowie Einbiegen / Kreuzen-Unfälle jeweils auf 15\% zu. In Bezug auf N3-Fahrzeuge zeigen Einbiegen / Kreuzen-Unfälle ebenfalls eine erhöhte Relevanz (14\%). Zudem steigt der Anteil der sonstigen Unfälle auf $13 \%$. Die höchste Relevanz für Unfälle im Längsverkehr ergibt sich für Sattelzugmaschinen (58\%), sicherlich auch bedingt durch den hohen Anteil von Autobahnunfällen.

\subsubsection{Fehlverhalten}

Die in der Bundesstatistik und in der UDB definierten Fehlverhalten sind weitestgehend vergleichbar. Die entsprechenden Verteilungen gehen aus Abb. 4-10 hervor. In den beiden dargestellten Säulen ist jeweils das unterste Segment datenbankspezifisch. Auf Seite der Destatis-Daten werden hier Fehlverhalten bezüglich der geltenden Beleuchtungsvorschriften (Anteil: $0,1 \%$ ) sowie andere Fehler zusammengefasst. Diese beiden Kategorien gibt es in den UDB-Daten nicht. Dies gilt umgekehrt für die Kategorie "Besondere Fahrmanöver“, 
welche in den Destatis-Auswertungen nicht vorkommt und das untere Segment der UDBSäule bildet. Das häufigste Fehlverhalten stellt in beiden Datensätzen ein Abstandsverstoß dar. Weitere relevante Fehlverhalten sind „Nicht angepasste Geschwindigkeit“, „Fehler beim Abbiegen, Wenden, usw.“ sowie „Vorfahrt, Vorrang“. Mit Hinblick auf die UDB ist zudem noch „Falsche Straßenbenutzung“ zu nennen.

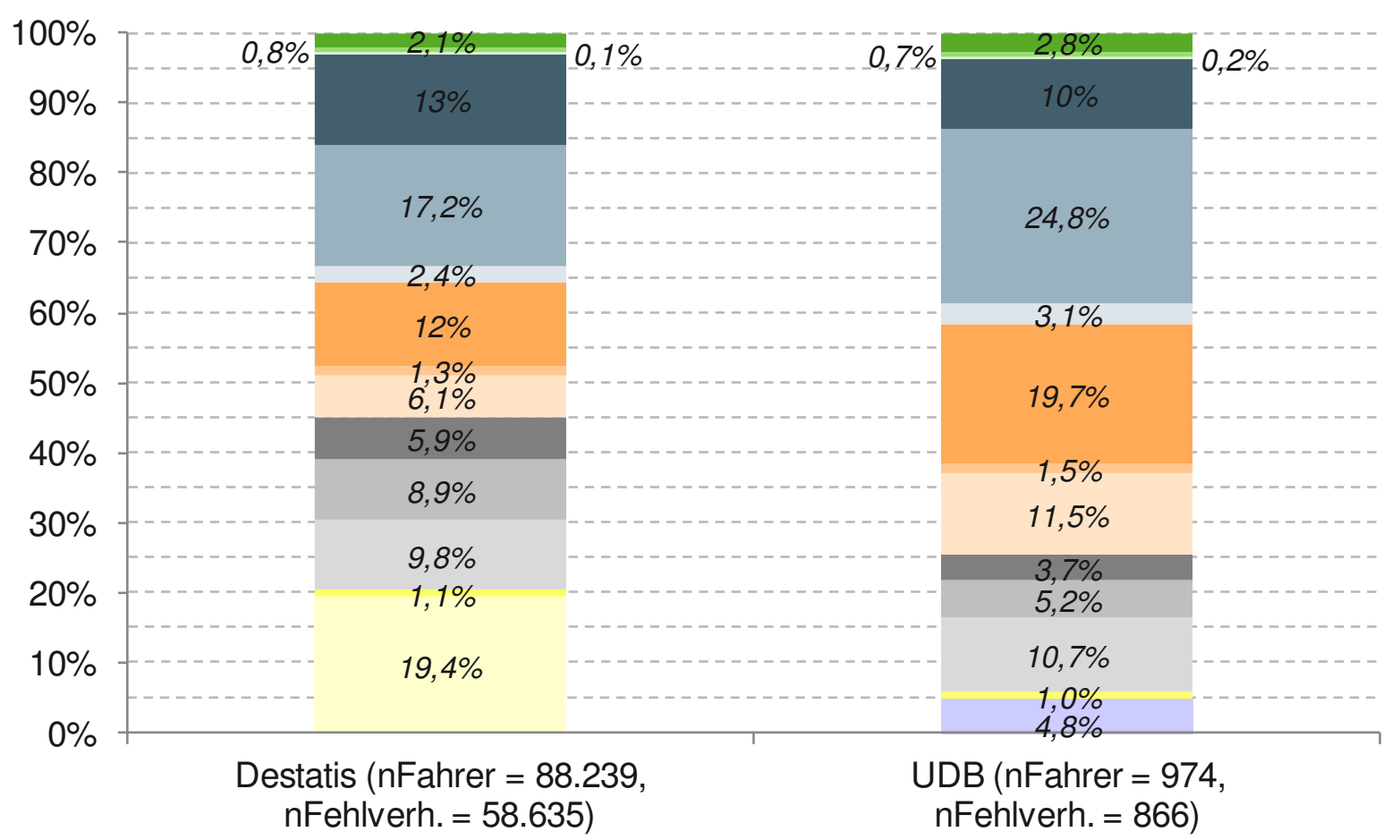

Fehlverhalten der an Unfällen mit Personenschaden beteiligten Fahrer von Güterkraftfahrzeugen mit zGG > 3,5 t

Verkehrstüchtigkeit (ohne Alkohol, Drogen)
Einfluss anderer berauschender Mittel
Abstand
Nicht angepasste Geschwindigkeit
Falsche Straßenbenutzung
Vorbeifahren, Nebeneinanderfahren
Ladung, Besetzung
Besondere Fahrmanöver (UDB)

Alkoholeinfluss

Fehler beim Abbiegen, Wenden, usw.

Falsches Verhalten geg. Fußgängern

Ruhender Verkehr, Verkehrssicherung

- Überholen

Vorfahrt, Vorrang

Beleuchtungsvorschriften \& Andere Fehler

Abb. 4-10: Vergleich Destatis (2010-2014) [STA11-15] \& UDB nach Fehlverhalten

Sowohl für N2 und N3-Fahrzeuge als auch für Sattelzugmaschinen bilden „Abstand“ und „Nicht angepasste Geschwindigkeit“" jeweils die häufigsten Fehlverhalten innerhalb der UDB. Allerdings weisen schwere Güterkraftfahrzeuge, d.h. N3-Fahrzeuge und Sattelzugmaschinen, gegenüber N2-Fahrzeugen häufiger „Fehler beim Abbiegen, Wenden, usw.“ auf.

\subsubsection{Unfallbeteiligte}

Der Pkw ist mit 55\% in den Destatis-Daten sowie 60\% in der UDB der mit Abstand häufigste Unfallgegner in Unfällen mit zwei Beteiligten, gefolgt von ungeschützten Verkehrsteilneh- 
mern, bei denen Radfahrer am häufigsten betroffen sind (Destatis: 13\%, UDB: 10\%). Alleinunfälle sind mit einem Anteil von $0,7 \%$ in der UDB prinzipbedingt unterrepräsentiert (Destatis: 8,5\%), da der Versicherer hier nicht eintritt, sofern kein Dritter geschädigt wird. Im Falle der in Abb. 4-11 dargestellten Verteilungen der Unfallgegner und Alleinunfälle besteht allerdings ein wesentlicher Unterschied zwischen der Destatis und der UDB-Auswertung. Während in den betrachteten UDB-Unfällen ein Unfallbeteiligter stets ein Güterkraftfahrzeug mit einem zGG größer 3,5 t ist, gilt diese Einschränkung für die Destatis-Auswertung der Unfallbeteiligten nicht. Anhand der öffentlich verfügbaren Daten ist eine Separierung nicht möglich, sodass hier auch sämtliche Unfälle von Güterkraftfahrzeugen mit einem zGG bis 3,5 $t$ in die Werte in Abb. 4-11 einfließen. Bei Unfällen zwischen zwei Güterkraftfahrzeugen kann der Unfallgegner allerdings in beiden Datenbanken ein zGG kleiner oder gleich 3,5 t aufweisen.

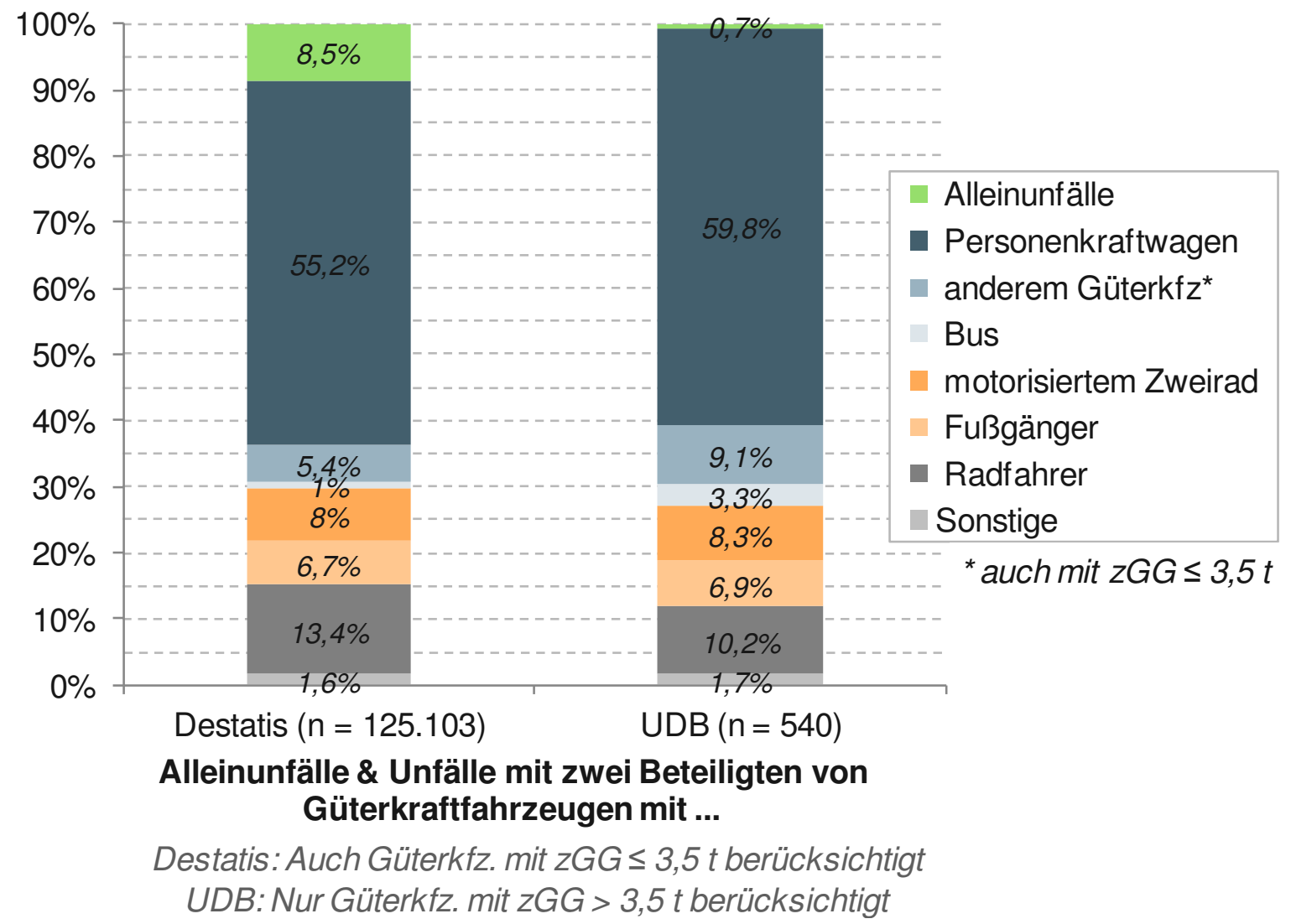

Abb. 4-11: Vergleich Destatis (2010-2014) [STA11-15] \& UDB nach Unfallbeteiligten

Ermittelt man die Verteilung der Unfallgegner in der UDB nur für N2-Fahrzeuge, so steigt der Pkw-Anteil nochmals auf 66\% (Anhang, Abb. 9-13). Zudem fallen die Anteile für Fußgänger und Busse höher aus, während die Relevanz motorisierter Zweiräder und anderer Güterkraftfahrzeuge abnimmt. Bei reinem Bezug auf N3-Fahrzeuge nehmen die Anteile der ungeschützten Verkehrsteilnehmer jeweils um ca. 1\% zu (Anhang, Abb. 9-14). Für Sattelzugmaschinen ergeben sich keine nennenswerten Unterschiede (Anhang, Abb. 9-15).

Betrachtet man ausschließlich die Anzahl der in Alleinunfällen und Unfällen mit zwei Beteiligten jeweils getöteten Personen, so ergeben sich deutlich erhöhte Anteile für Unfälle mit mo- 
torisierten Zweirädern sowie für Unfälle mit Fußgängern, welche ihren Anteil mehr als verdoppeln (Abb. 4-12). Während sich der Anteil von Unfällen mit anderen Güterkraftfahrzeugen bei Bezug auf Getötete in der UDB halbiert, nimmt er im Zuge der Destatis-Auswertungen von $5,4 \%$ auf $7,7 \%$ zu. Einen Grund hierfür könnten auch Unfälle zwischen zwei leichten Lkw, d.h. Lkw mit einem zGG bis 3,5 t, darstellen, welche in den ausgewerteten UDB-Daten nicht vorkommen.

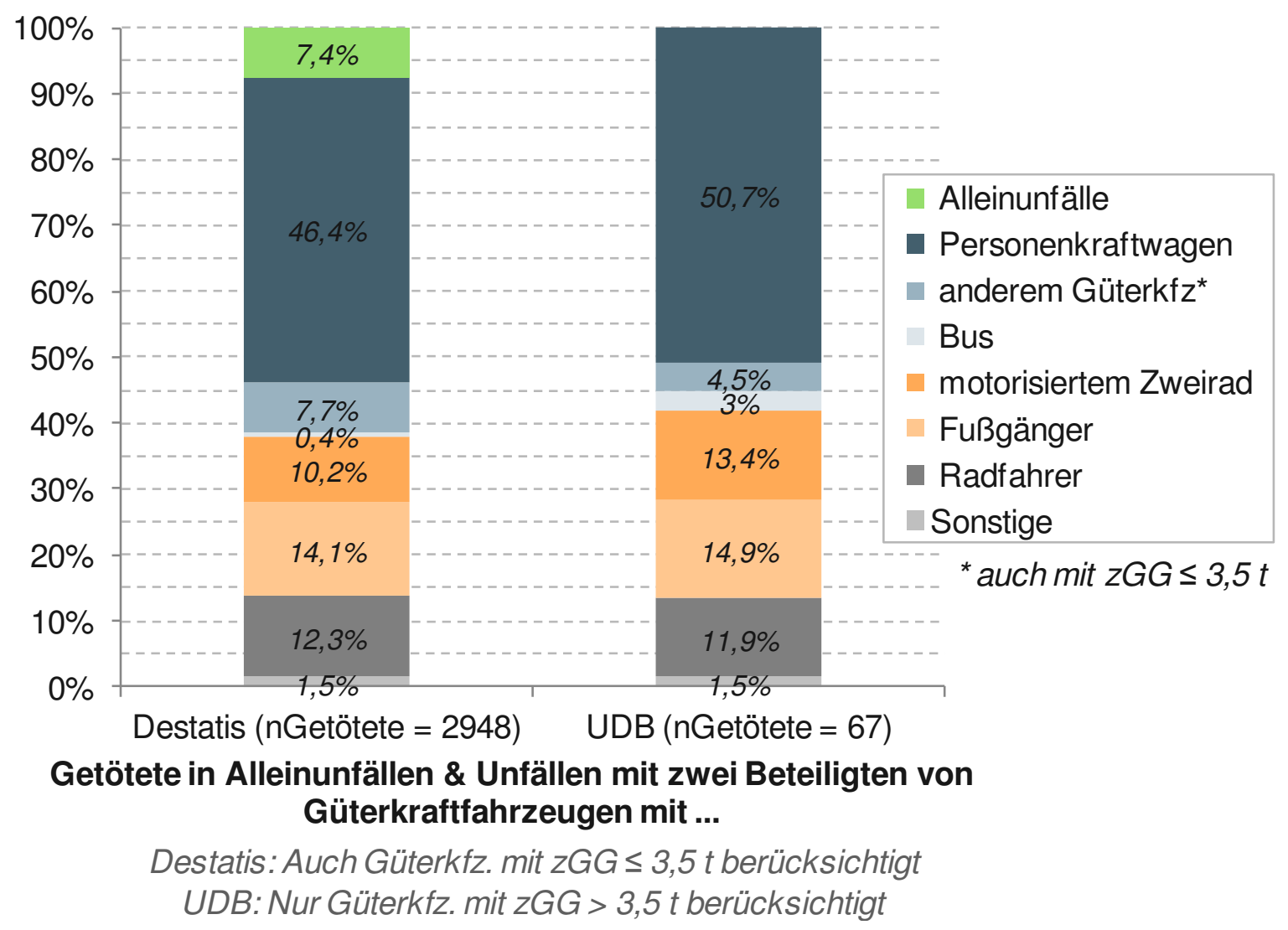

Abb. 4-12: Vergleich Destatis (2010-2014) [STA11-15] \& UDB nach Getöteten je Unfallbeteiligten

Die Zahl der Unfälle mit Zusammenstößen zwischen mehr als zwei beteiligten Verkehrsteilnehmern beträgt in den Destatis-Daten 31.026 und in der UDB 294. Für diese Unfälle weisen die Destatis-Daten 1130 und die UDB-Daten 46 Getötete auf. Die Verteilung der Unfallbeteiligten bei Berücksichtigung aller ausgewerteten UDB-Unfälle geht aus Abb. 9-16 im Anhang hervor und umfasst insgesamt 941 Unfallgegner. Für diese nimmt der Anteil von Güterkraftfahrzeugen gegenüber Abb. 4-11 stark zu (von 4,5\% auf 18\%). Gleichzeitig nehmen die Anteile der ungeschützten Verkehrsteilnehmer ab.

\subsubsection{Weitere Auswertungen der Unfalldatenbank der Versicherer}

Im Folgenden werden anhand der UDB-Daten weitere Auswertungen durchgeführt, die mittels der öffentlich verfügbaren Destatis-Daten nicht möglich sind. Dies umfasst Aspekte wie Fahreralter, Kollisionsart, Unfallursache, Fahrgeschwindigkeit oder Bremsverhalten. Zu- 
nächst werden die ausgewerteten UDB-Daten hinsichtlich des Alters und der Nationalität der an den Unfällen beteiligten Güterkraftfahrzeuge näher charakterisiert.

\subsubsection{Fahrzeugalter und Nationalität}

Für die 974 im Rahmen der UDB-Auswertung berücksichtigten Fahrer von Güterkraftfahrzeugen mit einem zGG größer 3,5 t liegt für 787 Fahrzeuge das Jahr der Erstzulassung vor. Von diesen wurden mehr als $70 \%$ zwischen 2000 und 2009 zugelassen, wie aus Abb. 4-13 hervorgeht. Zudem handelt es sich fast ausschließlich um deutsche Fahrzeuge (Anteil: 96\%). Ähnliches gilt für die Fahrer, bei denen über $93 \%$ eine deutsche Staatsbürgerschaft haben.

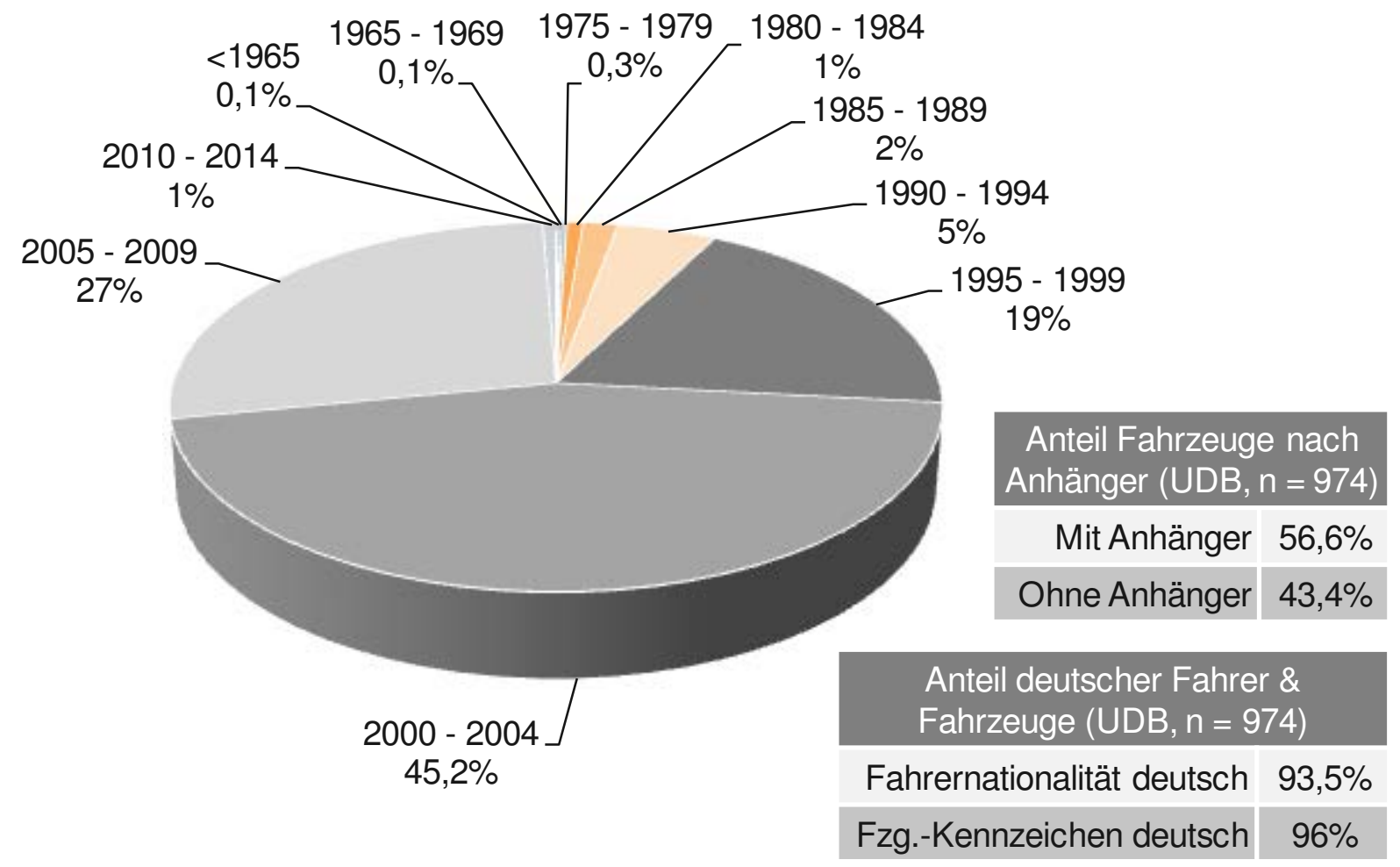

Abb. 4-13: An Unfällen beteiligte Güterkraftfahrzeuge mit zGG größer 3,5 t nach Jahr der Erstzulassung (UDB, $\mathrm{n}=787$ )

Die Mehrheit der an den UDB-Unfällen beteiligten Fahrzeuge führte zum Unfallzeitpunkt einen Anhänger mit sich (57\%). Dabei handelt sich in zwei Dritteln der Fälle um Sattelzugmaschinen. Ähnlich verhält es sich für die Destatis-Daten. Bezogen auf Liefer- und Lastkraftwagen mit einem zGG größer 3,5 t sowie Sattelzugmaschinen, für die ausnahmslos das Mitführen eines Aufliegers angenommen wird, verfügen hier $63 \%$ der an Unfällen zwischen 2010 und 2014 beteiligten Fahrzeuge $(n=73.066)$ über einen Anhänger. Dabei bleiben Liefer- und Lastkraftwagen ohne Angaben zum zGG bzw. deren Fahrer unberücksichtigt.

Verknüpft man das Jahr der Fahrzeugerstzulassung mit dem Unfalldatum, so lässt sich das Fahrzeugalter in Jahren zum Unfallzeitpunkt bestimmen. Die entsprechende Verteilung ist in Abb. 4-14 dargestellt. Demnach wiesen 65\% der Fahrzeuge zum Unfallzeitpunkt ein Alter von höchstens fünf Jahren auf. Insgesamt liegt der Durchschnittswert bei 5,2 Jahren. 


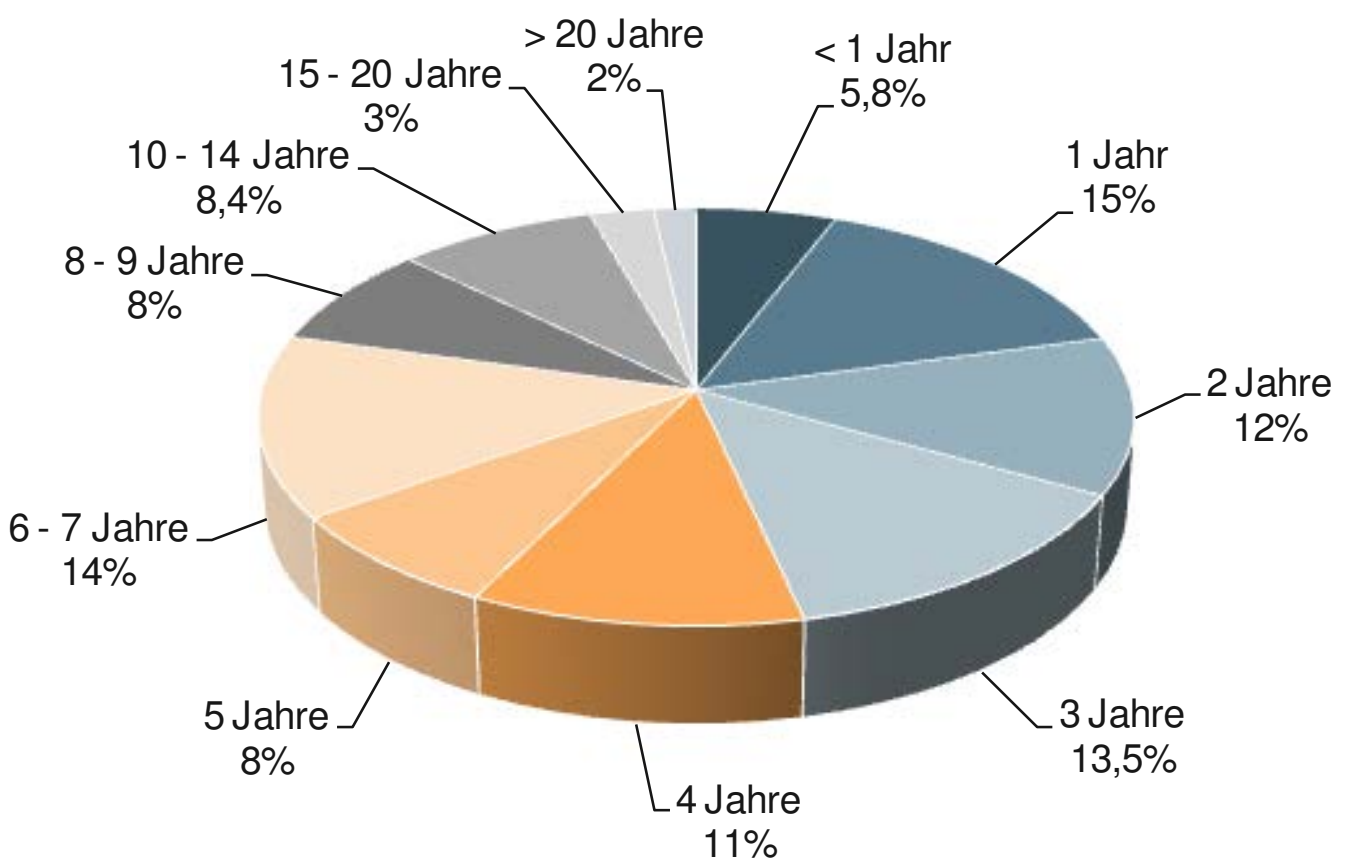

Abb. 4-14: An Unfällen beteiligte Fahrer von Güterkraftfahrzeugen mit zGG größer 3,5 t nach Fahrzeugalter zum Unfallzeitpunkt (UDB, $\mathrm{n}=787$ )

\subsubsection{Fahreralter und Dauer des Führerscheinbesitzes}

Das Alter ist für 837 der 974 betrachteten Fahrer bekannt. Gemäß Abb. 4-15 sind etwa die Hälfte dieser Fahrer zwischen 35 und 50 Jahren alt. Nur 5\% der Fahrer sind 60 Jahre oder älter. Bei Beschränkung auf Hauptverursacher zeigt sich das gleiche Bild (Abb. 9-17).

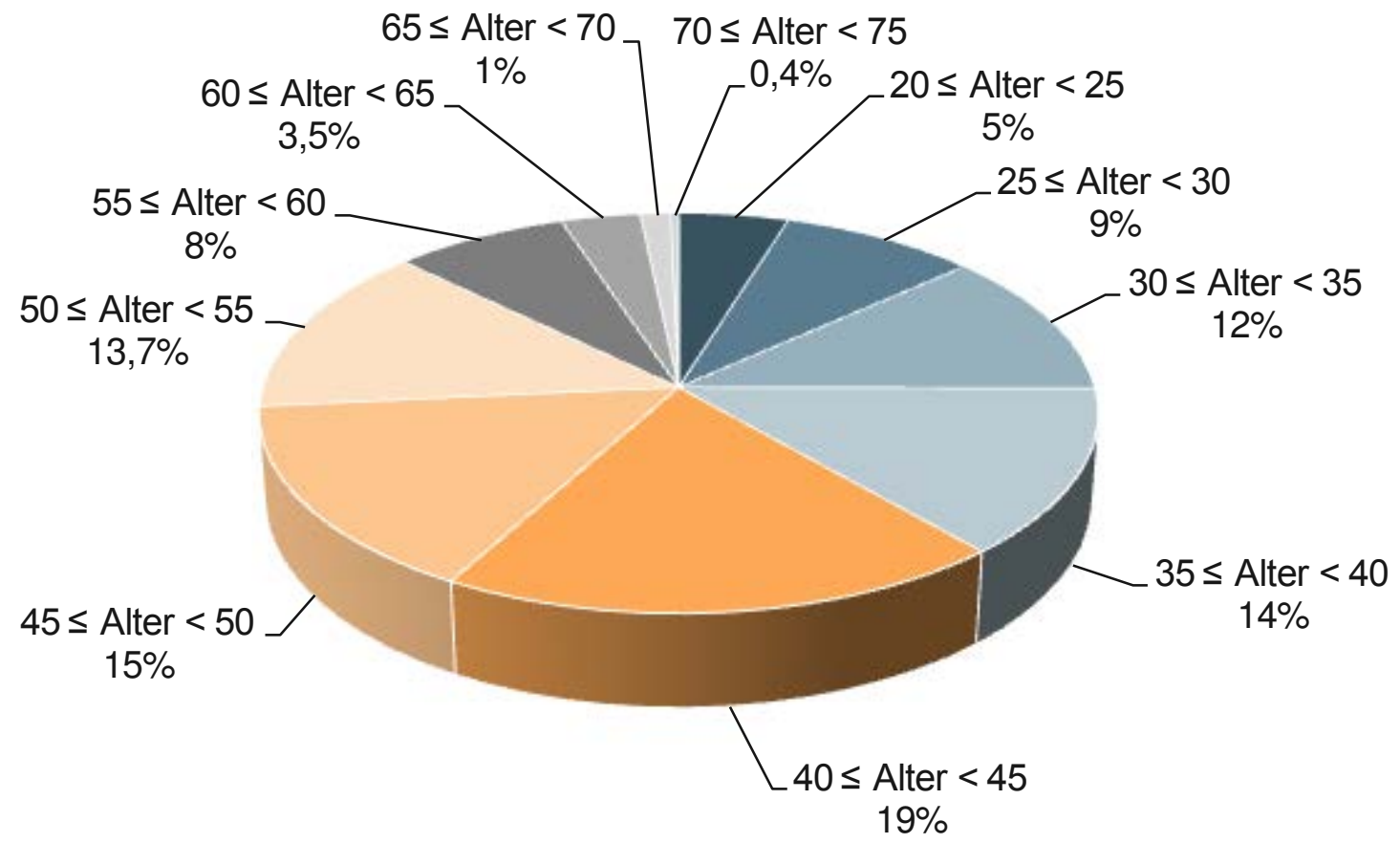

Abb. 4-15: An Unfällen beteiligte Fahrer von Güterkraftfahrzeugen mit zGG größer 3,5 t nach Fahreralter (UDB, $\mathrm{n}=837$ ) 
Neben dem Alter der Fahrer ist auch von Interesse, wie lange diese bereits im Besitz eines Lkw-Führerscheins sind. Das zugehörige Diagramm in Abb. 4-16 kombiniert dazu das Ausstellungsdatum der Fahrerlaubnis mit dem Unfalldatum. Fast die Hälfte der Fahrer war zum Unfallzeitpunkt höchstens 5 Jahre im Besitz des Führerscheins (Durchschnitt: 10,2 Jahre).

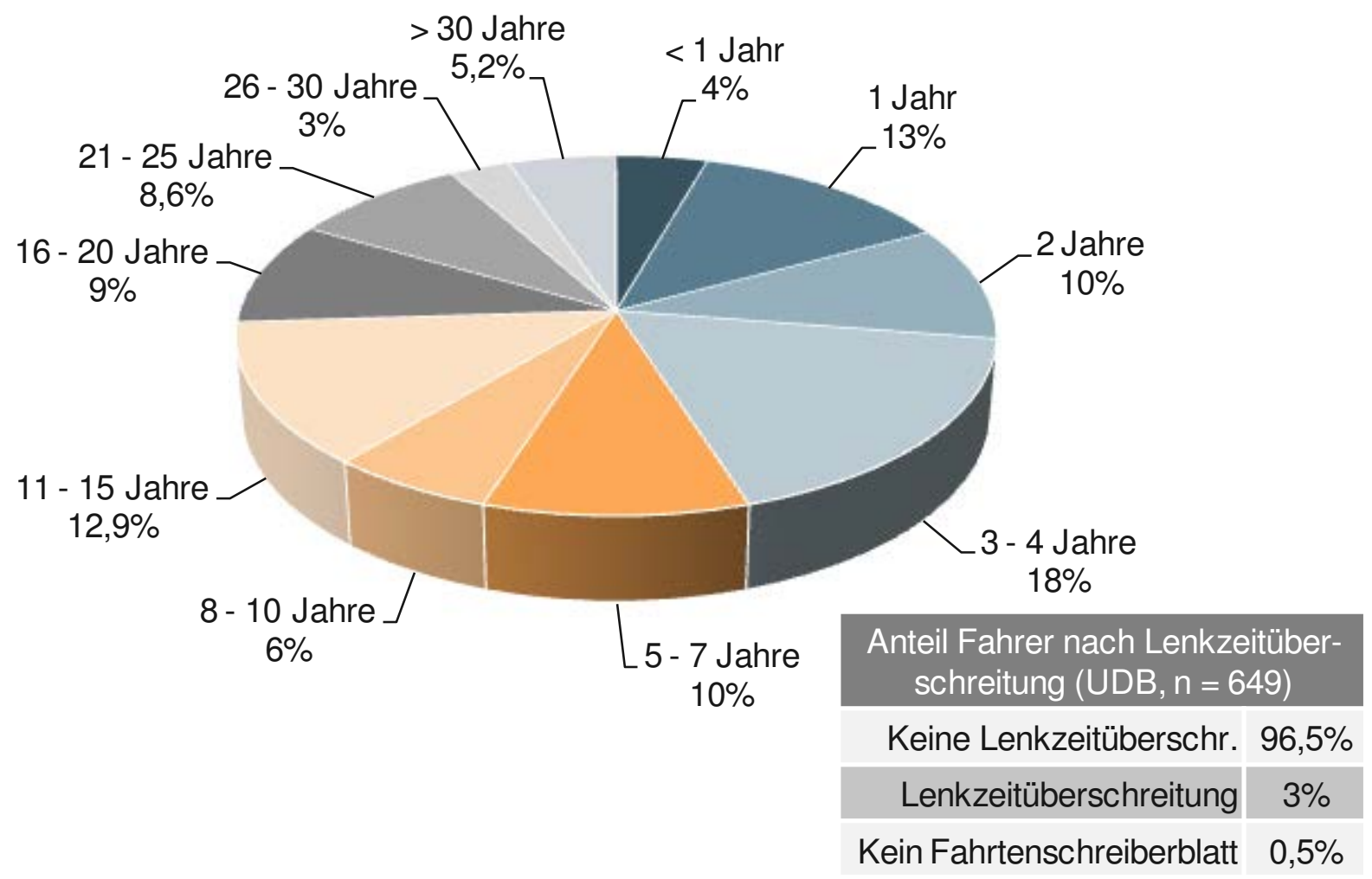

Abb. 4-16: An Unfällen beteiligte Fahrer von Güterkraftfahrzeugen mit zGG größer 3,5 t nach Dauer des Führerscheinbesitzes (UDB, $n=746$ )

Angaben zur Lenkzeitüberschreitung sind in der UDB für 649 Fahrer von Güterkraftfahrzeugen mit einem zGG von mehr als 3,5 t vorhanden bzw. zutreffend (für 48 Fahrer nicht zutreffend, für 275 Fahrer nicht vorhanden). Die überwiegende Mehrheit dieser Fahrer, d.h. 96,5\%, weist keine Lenkzeitüberschreitung auf.

\subsubsection{Kollisionsart}

Die Verteilung der Kollisionsarten wird im Folgenden nur für die jeweiligen Hauptverursacher ("Beteiligter 01" in der Verkehrsunfallanzeige) der beteiligten Fahrer von Güterkraftfahrzeugen mit zGG größer 3,5 t ermittelt. So wird vermieden, dass Unfälle mit mehreren Güterkraftfahrzeugen über 3,5 t zGG mehrfach in die Auswertung eingehen. Aus Abb. 4-17 wird ersichtlich, dass die Kollisionsart Front/Heck, also im Allgemeinen der Auffahrunfall, mit einem Anteil von $40 \%$ dominierend ist, gefolgt von Seitenkollisionen, welche insgesamt auf einen Anteil von 23\% kommt. Berücksichtigt man alle an Unfällen beteiligten Güterkraftfahrzeuge mit Angaben zur Kollisionsart ( $n=797$ ), so ändern sich die Anteile nur geringfügig. So kommt die Kollisionsart Front / Heck auf 43\% und der Seitenaufprall auf $21 \%$. Allerdings steigt die Relevanz von Frontalkollisionen (Front / Front) von $8 \%$ auf $12 \%$. 


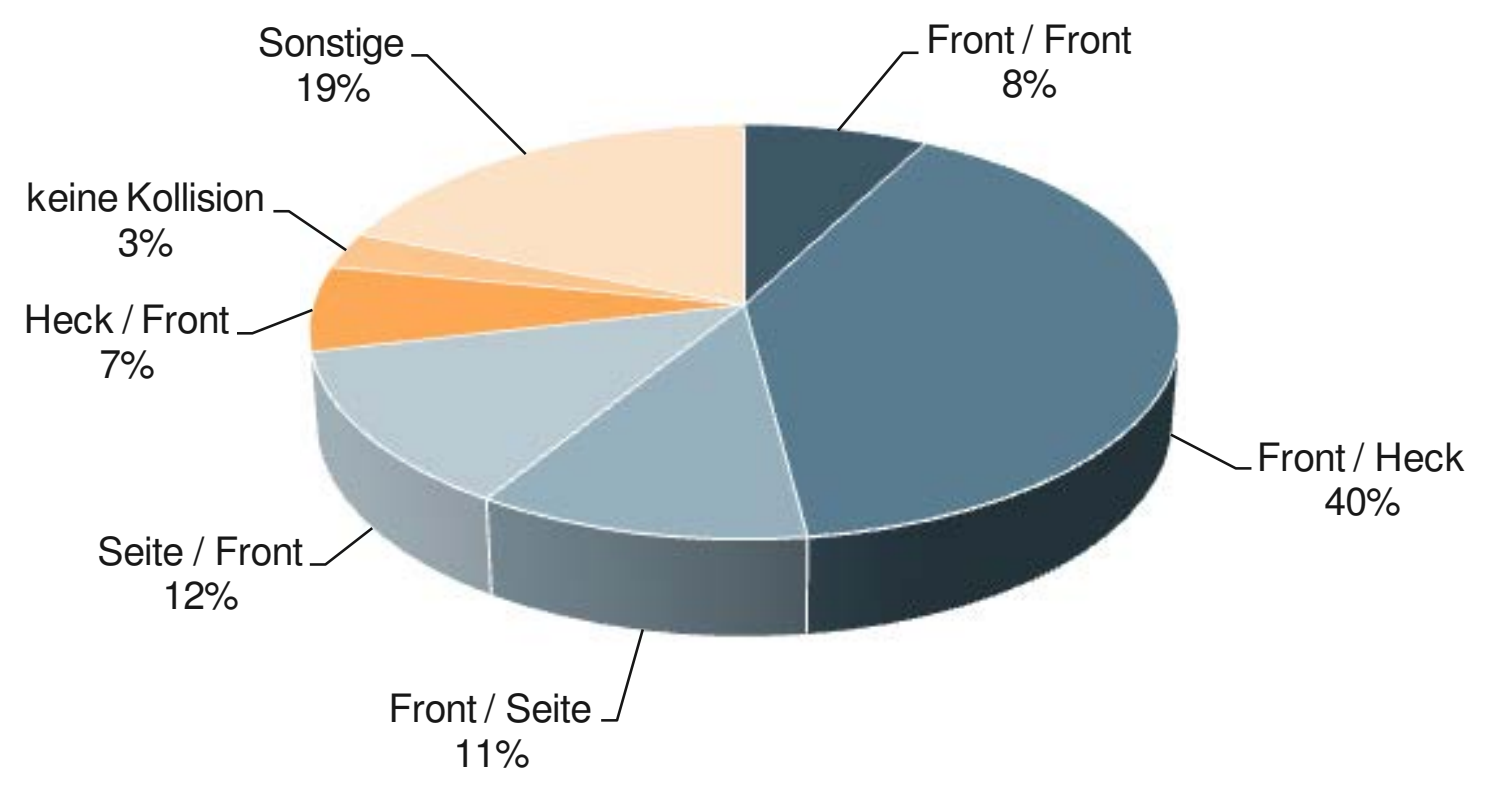

Abb. 4-17: Kollisionsart bei Güterkraftfahrzeugen mit zGG größer 3,5 t (UDB, $n=465)$, deren Fahrer Hauptverursacher (Beteiligter 01) ist

Betrachtet man ausschließlich Fahrer von N2-Fahrzeugen, so erhöht sich der Anteil von Auffahrunfällen für die Hauptverursacher auf 50\% (Anhang, Abb. 9-18). Zudem ergibt sich im Gegensatz zur Gesamtauswertung der Güterkraftfahrzeuge in Abb. 4-17 eine Diskrepanz zwischen den Kollisionsarten Front / Seite und Seite / Front. Für Erstgenannte nimmt der Anteil auf $9 \%$ ab während er für Letztere auf $16 \%$ ansteigt. Bezüglich N3-Fahrzeugen verhält es sich umgekehrt (Anhang, Abb. 9-19). Hier kommt die Kollisionsart Seite / Front nur auf einen Anteil von 6\%, wohingegen der Anteil für Front / Seite 13\% beträgt. N3-Fahrzeuge kollidieren tendenziell also häufiger frontal mit der Seite des Unfallgegners anstatt selbst seitlich getroffen zu werden, was entsprechend bei N2-Fahrzeugen häufiger der Fall ist. Bei den als Hauptverursacher eingestuften Fahrern von Sattelzugmaschinen zeigt sich im Vergleich zu Abb. 4-17 ein etwas niedrigerer Anteil für Auffahrunfälle (35\%) und mit 16\% ein erhöhter Anteil für die Kollisionsart Seite / Front (Anhang, Abb. 9-20), sodass auch Sattelzugmaschinen bzw. deren Auflieger in Seitenaufprallunfällen häufiger einen seitlichen Anstoß durch den Unfallgegner zu verzeichnen haben als selbst seitlich in den Unfallgegner hineinzufahren.

\subsubsection{Unfallursachen}

Gemäß der Definition des Statistischen Bundesamtes wird unterschieden nach allgemeinen Ursachen (u.a. Straßenverhältnisse, Witterungseinflüsse, Hindernisse), die dem Unfall und nicht einzelnen Beteiligten zugeordnet werden, sowie personenbezogenem Fehlverhalten (wie Vorfahrtsmissachtung, zu schnelles Fahren usw.), das bestimmten Fahrzeugführern oder Fußgängern, d.h. den Beteiligten zugeschrieben wird. Eine Übersicht über die allgemeinen Unfallursachen gibt Abb. 4-18. Für die 834 ausgewerteten Unfälle mit Güterkraftfahrzeugen über 3,5 t zGG sind diese in 54 Fällen ursächlich. Am häufigsten zu nennen sind dabei Schnee \& Eis sowie Sichtbehinderung durch Nebel oder blendende Sonne. Seitenwind als Ursache hat nur einen Anteil von $2 \%$. 


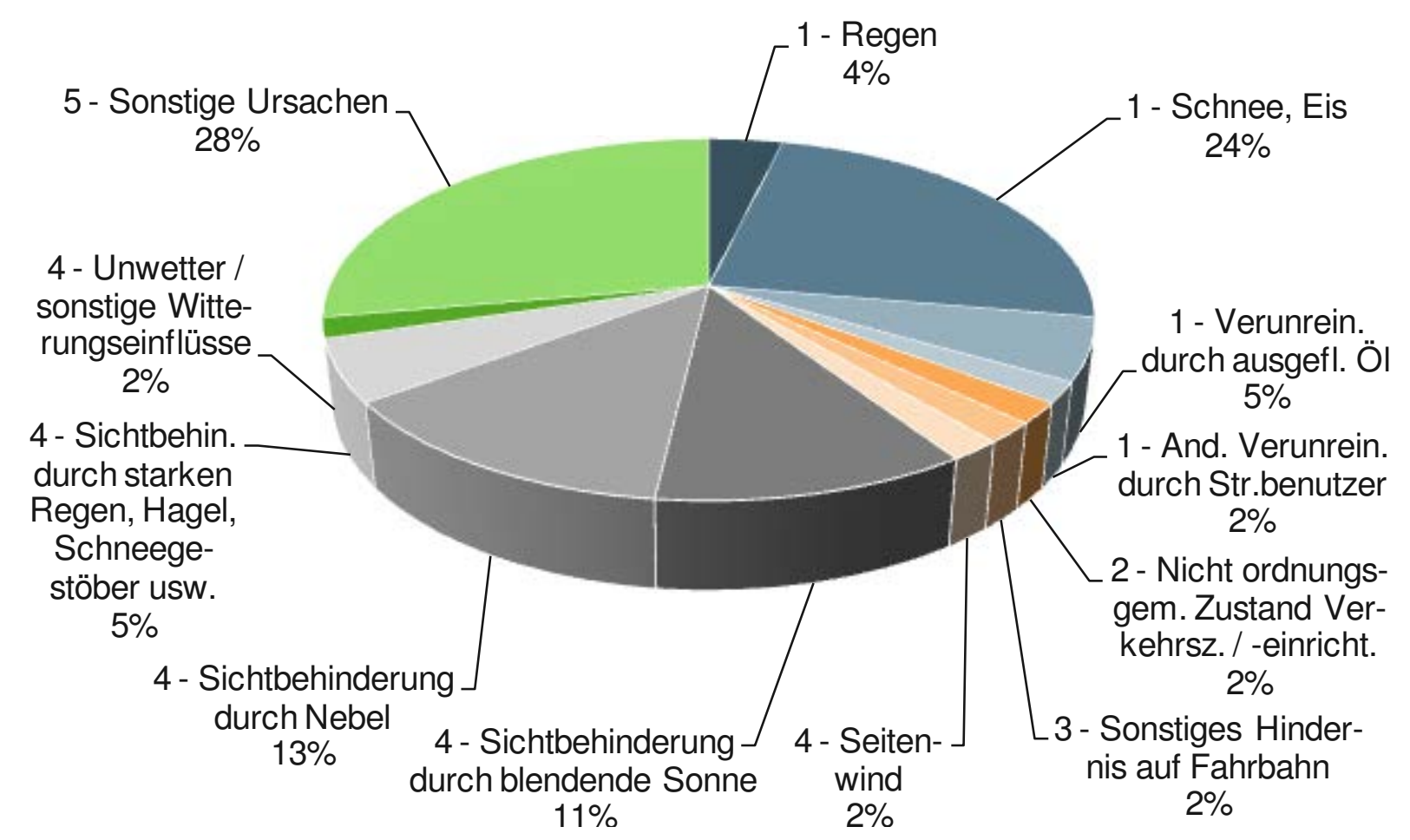

Abb. 4-18: Unfälle von Güterkraftfahrzeugen mit zGG größer 3,5 t nach festgestellter Unfallursache (UDB, $\left.n=834, n_{\text {Ursachen Verz. } 70-89}=54\right)$

Die Verteilung der als Unfallursachen eingestuften personenbezogenen Fehlverhalten geht aus Abb. 4-19 hervor. Für die 974 betrachteten Fahrer ergeben sich 644 Fehlverhalten.

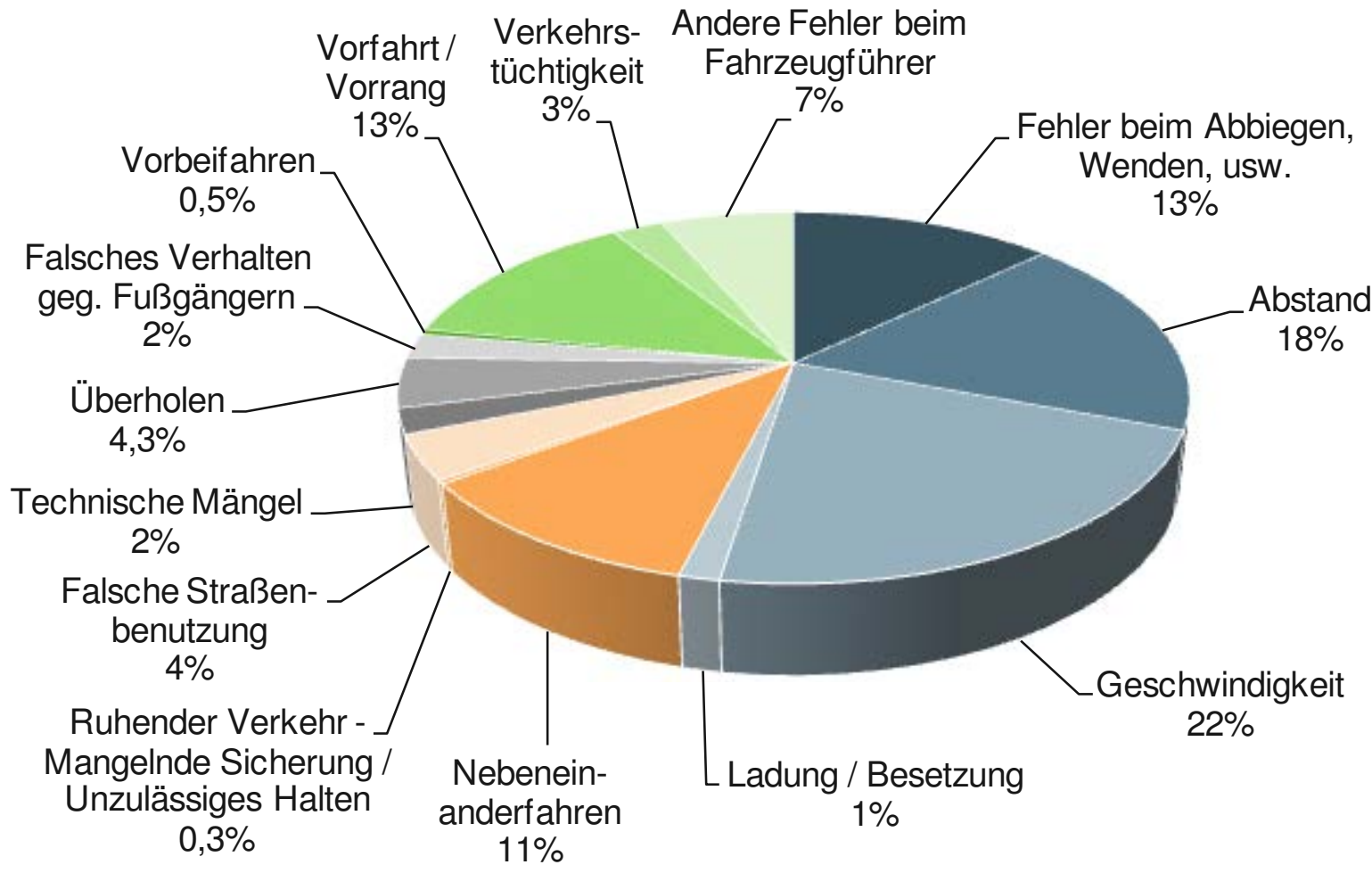

Abb. 4-19: An Unfällen beteiligte Fahrer von Güterkraftfahrzeugen mit zGG größer 3,5 t nach Unfallursache (UDB, $\left.n_{\text {Fahrer }}=974, n_{\text {Ursachen Verz. } 01-69}=644\right)$ 
Am häufigsten werden Geschwindigkeits- und Abstandsvergehen genannt. Relevant sind zudem Vorfahrtsmissachtungen und Fehler beim Abbiegen und Wenden sowie ein fehlerhaftes Wechseln des Fahrstreifens beim Nebeneinanderfahren oder ein Nichtbeachten des Reißverschlussverfahrens. Technische Mängel und unzureichende Ladungssicherung werden demgegenüber selten als Unfallursache in den UDB-Daten identifiziert. Hinsichtlich der Verkehrstüchtigkeit ist vor allem Übermüdung zu nennen. Alkoholeinfluss spielt kaum eine Rolle und macht bei Bezug auf die Gesamtzahl der unfallursächlichen Fehlverhalten nur einen Anteil von $0,3 \%$ aus.

\subsubsection{Fahrgeschwindigkeit und Bremsverhalten}

Hinsichtlich der Fahrgeschwindigkeit sind in erster Linie die als Hauptverursacher (Beteiligter 01) eingestuften Fahrer von Interesse. Diese weisen zu über $40 \%$ eine Fahrgeschwindigkeit oberhalb von $70 \mathrm{~km} / \mathrm{h}$ auf (Abb. 4-20).

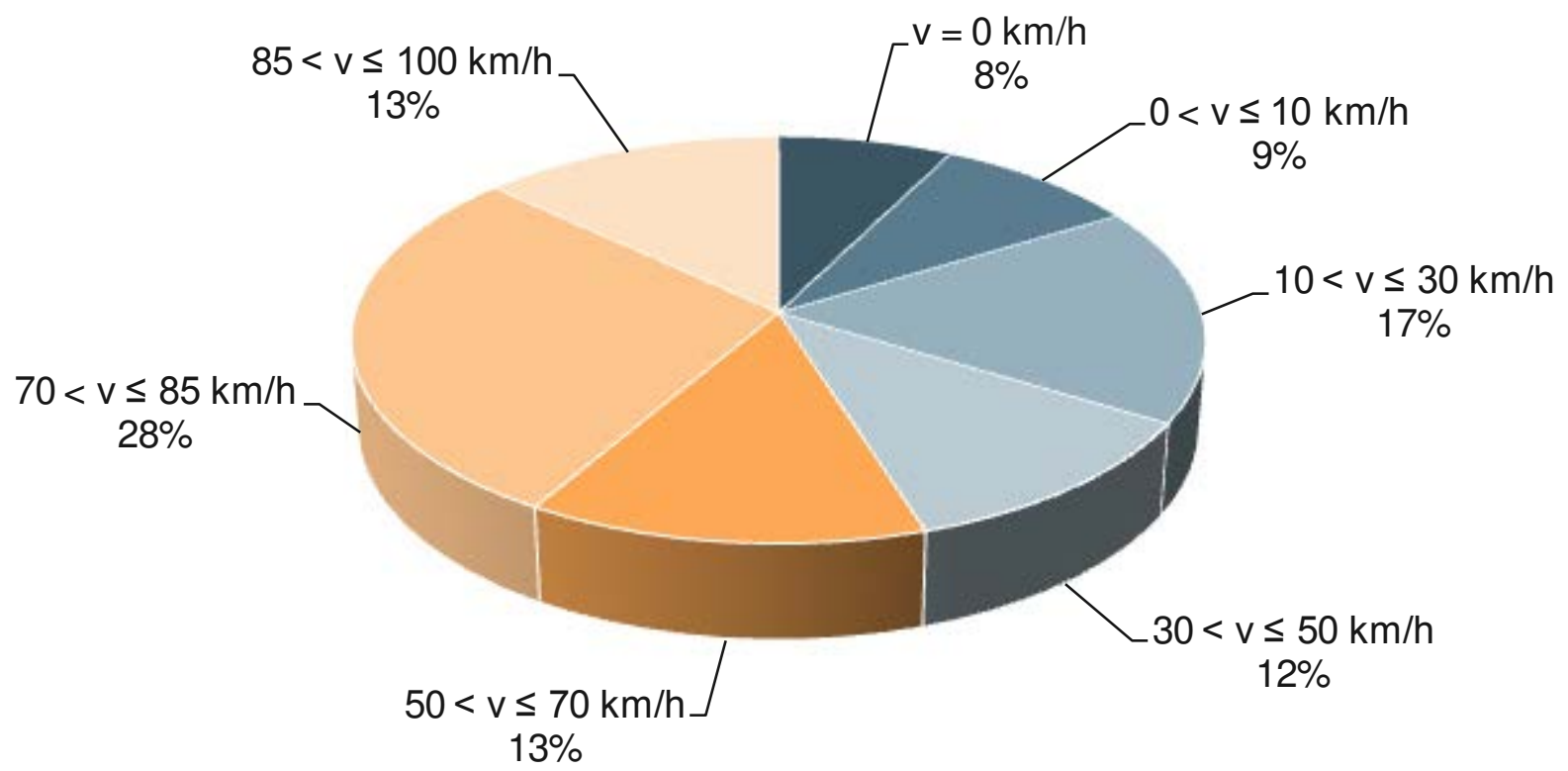

Abb. 4-20: Als Unfallhauptverursacher eingestufte Fahrer von Güterkraftfahrzeugen mit zGG größer 3,5 t und bekannter Fahrgeschwindigkeit (UDB, $\mathrm{n}=306$ )

Auffällig sind die Ergebnisse zum Bremsverhalten. So bremsen fast 70\% der als Hauptverursacher eingestuften Güterkraftfahrzeugfahrer, für die Informationen zum Bremsverhalten vorliegen ( $n=502)$, vor dem Unfall nicht (Abb. 4-21). Bezogen auf Autobahnunfälle liegt die Quote bei ca. 65\%. Aus Abb. 4-21 geht zudem das Bremsverhalten der Fahrer von Güterkraftfahrzeugen mit zGG größer 3,5 $\mathrm{t}$ in Unfällen mit ungeschützten Verkehrsteilnehmern hervor, für die der Anteil von Fällen ohne vorherige Bremsung des Lkw-Fahrers sogar bei insgesamt $75 \%$ liegt. Bei genauerer Aufschlüsselung wird allerdings deutlich, dass hier vor allem Unfälle mit Radfahrern auffällig sind. In den ausgewerteten Fällen haben 86\% der LkwFahrer keine vorherige Bremsung durchgeführt. Für motorisierte Zweiräder liegt diese Quote bei $72 \%$, während in den Fußgängerunfällen der Anteil der Lkw-Fahrer ohne vorherige Bremsung bei $63 \%$ liegt. Ein möglicher Grund für diese Unterschiede liegt in der höheren Eigen- 
geschwindigkeit der Zweiradfahrer oder ihrer eingeschränkten bzw. verspäteten Wahrnehmbarkeit durch den Güterkraftfahrzeugfahrer.

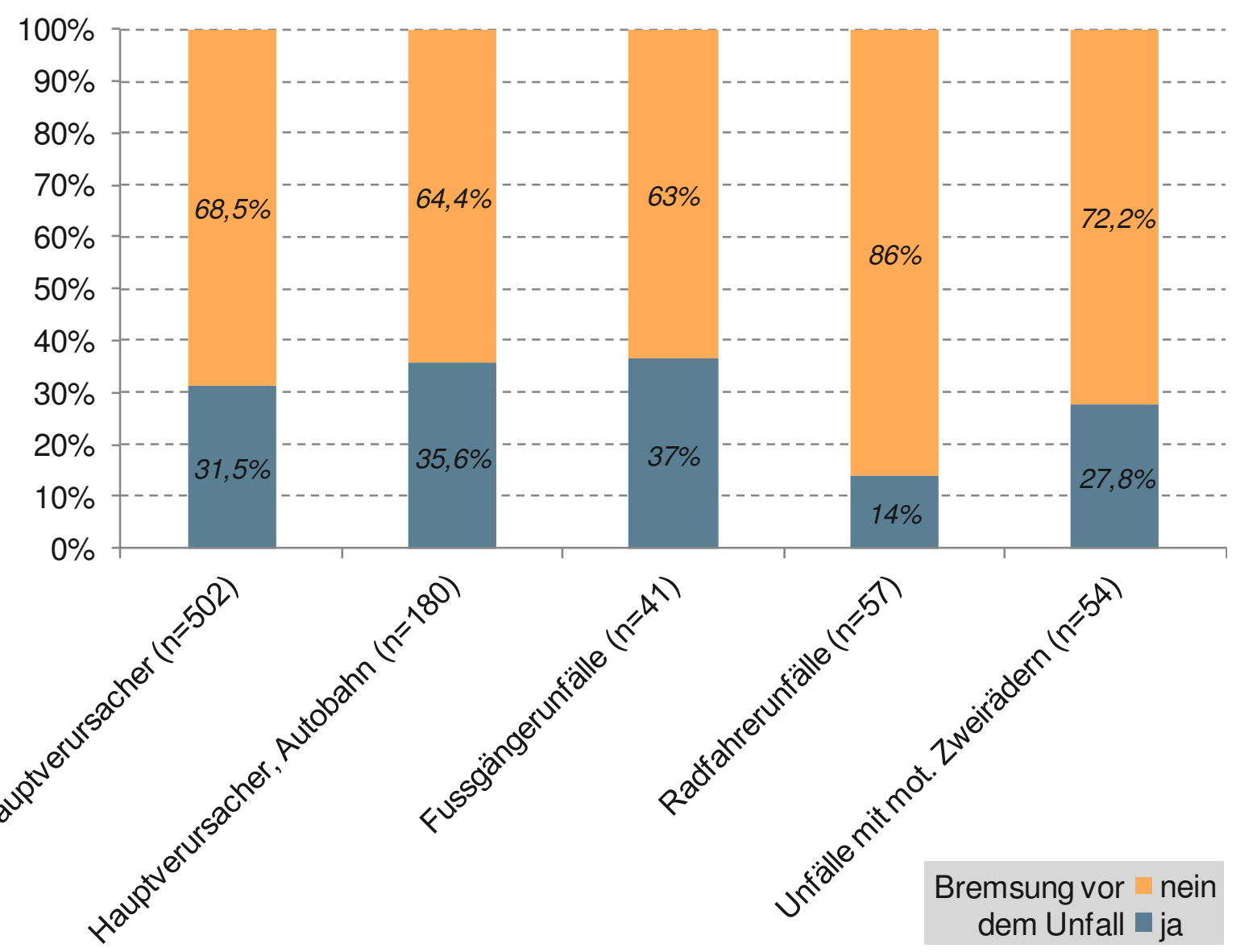

Abb. 4-21: An Unfällen beteiligte Fahrer von Güterkraftfahrzeugen mit zGG größer 3,5 t nach Bremsung vor dem Unfall (UDB, $\mathrm{n}=855$ )

\subsubsection{Seite des Hauptanstoßes}

Neben der Art der Kollision (Kapitel 4.1.2.3) lässt sich mit Hilfe der UDB-Daten auch die Seite des Hauptanstoßes bestimmen. Eine entsprechende Auswertung für die als Hauptverursacher eingestuften Fahrer von Güterkraftfahrzeugen mit zGG größer 3,5 t geht aus Abb. 4-22 hervor. Der Hauptanstoß ist, sofern mehrere Kollisionen vorliegen, in der Regel die schwerwiegendere Kollision, d.h. die mit dem höheren Verletzungspotential für den Beteiligten. Es wird in der UDB zwischen Primär- und Sekundäranstoß unterschieden, weitere Kollisionen werden für einen Beteiligten nicht erfasst. Der Primäranstoß beschreibt dabei die zeitlich zuerst erfolgte Kollision. Ferner wird vermerkt, ob jeweils der Primär- oder der Sekundäranstoß den Hauptanstoß bilden oder ob für den Beteiligten kein Hauptanstoß vorliegt. Für die Hauptverursacher unter den beteiligten Fahrern von Güterkraftfahrzeugen mit zGG größer 3,5 t liegen in den UDB-Daten in 577 Fällen Angaben zum Hauptanstoß vor. Hierbei ist der Primäranstoß zu 80\% auch der Hauptanstoß, während der Anteil des Sekundäranstoßes bei 4\% liegt (Anhang, Abb. 9-21). In 16\% der Fälle liegt die Angabe „kein Anstoß“ vor. In Abb. 4-22 zeigt sich, dass hinsichtlich der Seite des Hauptanstoßes der Frontbereich mit 
$68 \%$ dominierend ist. Ein seitlicher Anstoß erfolgt fast doppelt so häufig an der rechten wie an der linken Seite.

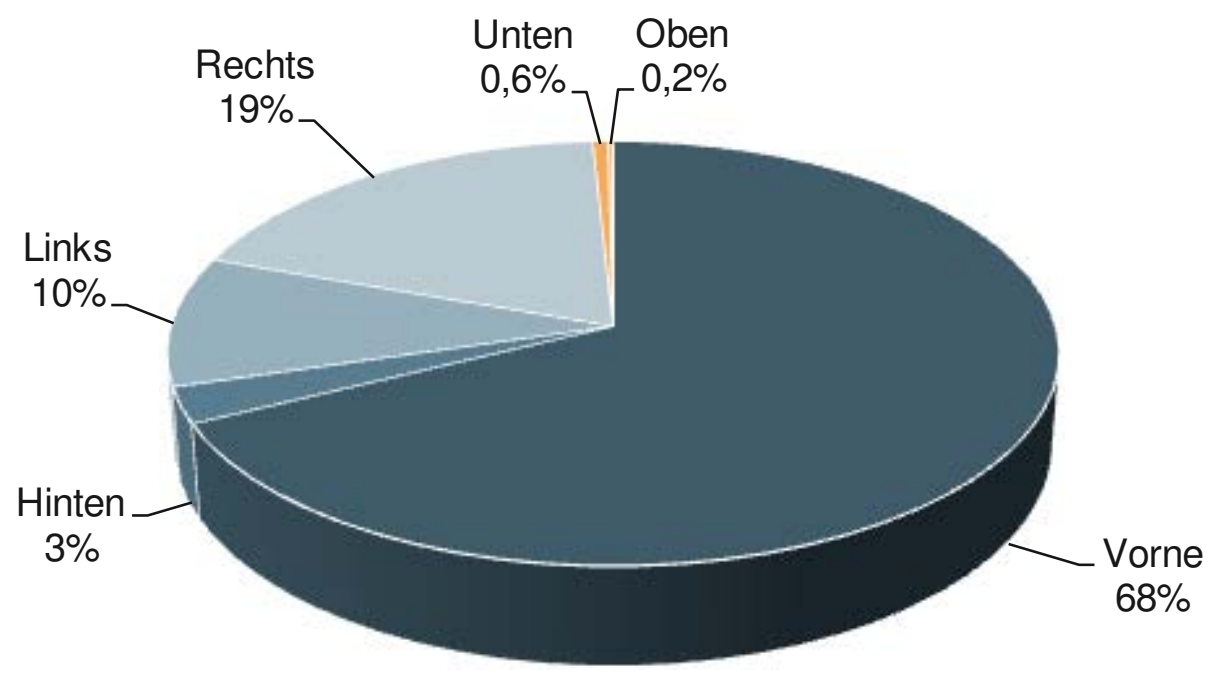

Abb. 4-22: Seite des Hauptanstoßes bei Güterkraftfahrzeugen mit zGG größer 3,5 t (UDB, $\mathrm{n}=474$ ), deren Fahrer Hauptverursacher (Beteiligter 01) ist

Die oben beschriebene Verteilung der Hauptanstoßseiten, d.h. Frontbereich dominierend und ein deutlich höherer Anteil der rechten Fahrzeugseite bei seitlichen Anstößen, bleibt auch bei getrennter Betrachtung von N2-, N3- oder Sattelzugfahrzeugen bestehen (Anhang, Abb. 9-22, Abb. 9-23 \& Abb. 9-24). Während ein Hauptanstoß im Heckbereich bei N2- und N3-Fahrzeugen einen Anteil von 5\% bzw. $4 \%$ hat, spielt dieser bei Sattelzugmaschinen mit $0,5 \%$ kaum eine Rolle.

\subsubsection{7 Überdeckungsgrad}

Angaben zum Grad der Überdeckung beziehen sich ausschließlich auf Frontal- und Heckkollisionen. Dieser beschreibt den im Rahmen einer Kollision mit einem Unfallgegner oder einem Hindernis getroffenen Teil der Front bzw. des Hecks in Bezug auf die Fahrzeugbreite. In den UDB-Daten werden insgesamt vier Überdeckungsgrade unterschieden, d.h. Viertelüberdeckung (1/4), halbe Überdeckung (1/2), Dreiviertelüberdeckung (3/4) und volle Überdeckung (1). Für die in Frontalkollisionen als Hauptverursacher eingestuften Güterkraftfahrzeuge mit zGG größer 3,5 t ist die Verteilung in Abb. 4-23 dargestellt. Fast $40 \%$ der betrachteten Fälle zeigen nur eine Viertelüberdeckung, welche mit Hinblick auf den Insassenschutz (Deformationen, Intrusionen \& Energieabbau), deutlich kritischer zu sehen ist als ein Unfall mit voller Überdeckung. Dieser hat mit 34\% den zweitgrößten Anteil in den UDB-Daten.

Beschränkt man die Auswertung allein auf N2-Fahrzeuge, so macht der Unfall mit voller Überdeckung den größten Anteil aus, bei gleichzeitiger Abnahme der Anteile von halber und Dreiviertelüberdeckung (Anhang, Abb. 9-25). N3-Fahrzeuge weisen mit 42\% den höchsten Anteil für Kollisionen mit Viertelüberdeckung auf (Anhang, Abb 9-26). Demgegenüber zeigen Sattelzugmaschinen im Vergleich zu den beiden vorgenannten Fahrzeugklassen einen fast doppelt so hohen Anteil für Kollisionen mit Dreiviertelüberdeckung (Anhang, Abb. 9-27). 


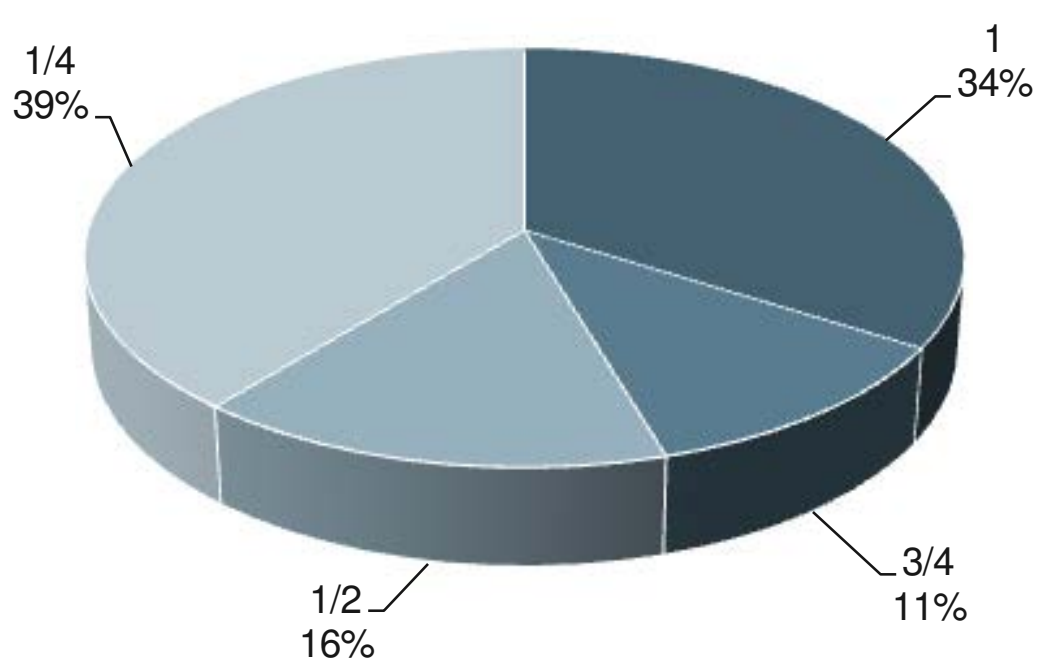

Abb. 4-23: Überdeckungsgrad beim Frontalanprall bei Güterkraftfahrzeugen mit zGG größer $3,5 \mathrm{t}(\mathrm{UDB}, \mathrm{n}=210)$, deren Fahrer Hauptverursacher (Beteiligter 01) ist

\subsubsection{MAIS-Verletzungsschweregrad}

Die AIS-Skala (Abbreviated Injury Scale) umfasst sechs Schweregrade für Einzelverletzungen, welche sich in unverletzt (AIS 0), gering bis mäßig (AIS 1-2), schwer bis kritisch (AIS 3-5) sowie nicht überlebbar (AIS 6) gliedern lassen [KRA09]. Eine unbekannte Verletzungsschwere wird mit AIS 9 kodiert. Die Gesamtverletzungsschwere einer Person wird häufig als maximaler AIS-Wert (MAIS) angegeben. Dies ist der höchste bei einer Person mit Mehrfachverletzungen vorgefundene Schweregrad. Hinsichtlich der UDB-Daten ergibt die Auswertung der MAIS-Verletzungsschweregrade, dass die Hälfte der MAIS 1 bis 9 eingestuften Verunglückten $(n=1176)$ nur MAIS 1 verletzt sind (Abb. 4-24). In Bezug auf die MAIS 1bis MAIS 6-Fälle $(n=948)$ sind $21 \%$ der Verunglückten mindestens MAIS 3 verletzt. Für $20 \%$ der Verletzten ließ sich der Gesamtverletzungsschweregrad nicht bestimmen.

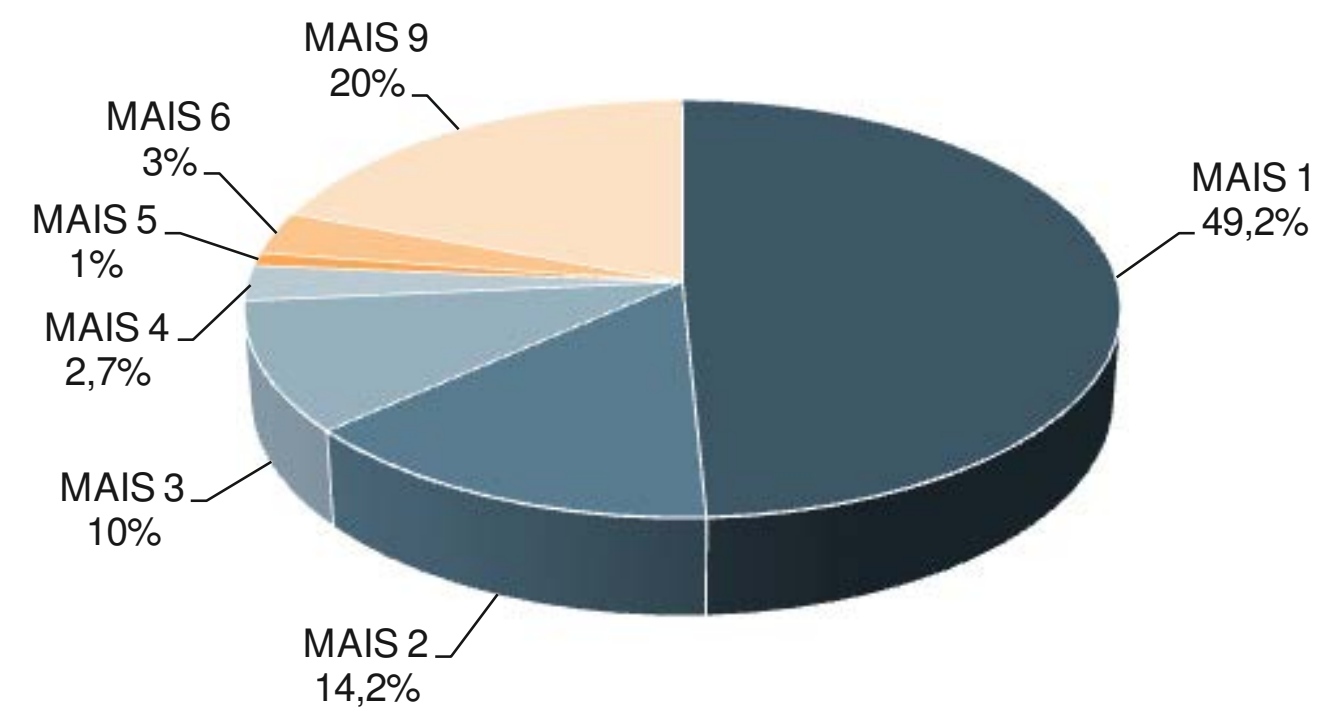

Abb. 4-24: Unfälle von Güterkraftfahrzeugen mit zGG größer 3,5 t nach MAIS-Verletzungsschweregrad der Beteiligten (UDB, $n_{\text {Unfälle }}=834$, $\mathrm{n}_{\text {Verletzte }}=1176$ ) 
Die Zahl der unverletzten Beteiligten (MAIS 0) an Unfällen von Güterkraftfahrzeugen mit zGG größer 3,5 t in der UDB beträgt 958.

\subsubsection{Fazit}

Zusammenfassend bleibt für Güterkraftfahrzeuge mit einem zGG größer 3,5 $t$ festzuhalten, dass in der Bundesstatistik ca. ein Drittel der Fahrzeuge an einem Unfall auf der Autobahn beteiligt sind. Dieser Anteil ist für die UDB mit $43 \%$ höher. Sattelzugmaschinen bilden die größte Gruppe innerhalb der Destatis und UDB-Daten. Bei Beschränkung der Auswertung nach Fahrzeugart auf Unfälle mit Getöteten zeigt sich jeweils eine Zunahme der Anteile für schwere Güterkraftfahrzeuge. Allerdings sind in den UDB-Daten Unfälle mit Leichtverletzten im Vergleich zur Bundesstatistik weniger stark vertreten. Generell sind die beteiligten Fahrer aller Güterkraftfahrzeuge mit einem zGG größer 3,5 t in etwa der Hälfte der Unfälle mit Personenschaden auch Hauptverursacher.

Der Zusammenstoß mit einem vorausfahrenden / wartenden Fahrzeug bildet die häufigste Unfallart (Destatis: 27\%, UDB: 34\%), gefolgt vom Zusammenstoß mit einem einbiegenden / kreuzenden Fahrzeug (Destatis: 17\%, UDB: 19\%). Beschränkt man die Auswertung auf Unfälle mit Getöteten, so zeigt sich sowohl für die Destatis als auch für die UDB-Daten eine deutliche Erhöhung der Anteile für Zusammenstöße mit einem entgegenkommenden Fahrzeug sowie für Zusammenstöße zwischen Fahrzeug und Fußgänger. Die entsprechenden Anteile von Radfahrern und motorisierte Zweirädern sind in den UDB-Daten mit dem Fußgängeranteil vergleichbar. Sowohl für die Destatis-Daten als auch für die UDB stellt der Unfall im Längsverkehr den mit Abstand häufigsten Unfalltypen dar. Dieser trifft jeweils auf ca. die Hälfte der an Unfällen mit Personenschaden beteiligten Güterkraftfahrzeuge zu.

Das häufigste Fehlverhalten stellt generell ein Abstandsverstoß dar. Weitere relevante Fehlverhalten sind „Nicht angepasste Geschwindigkeit“, „Fehler beim Abbiegen, Wenden, usw.“ sowie „Vorfahrt, Vorrang“. Der Pkw ist mit 55\% in den Destatis-Daten sowie 60\% in der UDB der mit Abstand häufigste Unfallgegner, gefolgt von ungeschützten Verkehrsteilnehmern, bei denen Radfahrer am häufigsten betroffen sind. Betrachtet man ausschließlich die Anzahl der in Alleinunfällen und Unfällen mit zwei Beteiligten jeweils getöteten Personen, so ergeben sich deutlich höhere Anteile für Unfälle mit motorisierten Zweirädern sowie für Unfälle mit Fußgängern. Letztere zeigen einen mehr als doppelt so hohen Anteil.

Im Rahmen der weiteren Auswertungen der UDB wird ersichtlich, dass die Kollisionsart Front / Heck, also der klassische Auffahrunfall, mit einem Anteil von 40\% dominierend ist, gefolgt von Seitenkollisionen, welcher insgesamt auf einen Anteil von 23\% kommt. Als die häufigsten allgemeinen Unfallursachen sind Schnee \& Eis sowie Sichtbehinderung durch Nebel oder blendende Sonne zu nennen. Hinsichtlich der als Unfallursache eingestuften personenbezogenen Fehlverhalten kommen Geschwindigkeits- und Abstandsvergehen am häufigsten vor, welche bei der Unfallaufnahme sicherlich auch häufig in Fällen angeführt werden, bei denen sich die genaue Ursache nicht unmittelbar ermitteln lässt. Technische Mängel und unzureichende Ladungssicherung werden demgegenüber selten als Unfallursache innerhalb 
der UDB-Daten identifiziert. Hinsichtlich der Verkehrstüchtigkeit ist vor allem Übermüdung zu nennen. Alkoholeinfluss spielt hier kaum eine Rolle.

Auffällig sind die Ergebnisse zum Bremsverhalten. Fast 70\% der als Hauptverursacher eingestuften Fahrer von Güterkraftfahrzeugen über 3,5 t zGG mit bekanntem Bremsverhalten bremsen vor dem Unfall nicht. In Unfällen mit ungeschützten Verkehrsteilnehmern liegt der Anteil sogar bei insgesamt $75 \%$. Hier sind vor allem Unfälle mit Radfahrern auffällig. Hinsichtlich der Seite des Hauptanstoßes ist der Frontbereich mit 68\% dominierend. Ein seitlicher Anstoß erfolgt fast doppelt so häufig an der rechten wie an der linken Seite. Des Weiteren zeigen fast $40 \%$ der Frontal- und Heckkollisionen nur eine Überdeckung von $25 \%$.

\subsection{Leicht-Lkw}

Ein besonderer Fokus dieses Projekts liegt auf 12 t-Leicht-Lkw-Kombinationen (Kapitel 2.2). Das Unfallgeschehen dieser leichten Gliederzüge soll im Folgenden anhand der Auswertung einer entsprechenden fahrzeugspezifischen Sonderabfrage beim Statistischen Bundesamt für die Jahre 2010 bis 2013 näher charakterisiert werden.

\subsubsection{Sonderabfrage in der amtlichen Statistik}

Die zu untersuchenden Leicht-Lkw-Kombinationen lassen sich in den Daten der amtlichen Verkehrsunfallstatistik nicht direkt und eindeutig identifizieren. Hier müssen, auch unter Berücksichtigung von Randbedingungen der Datenerhebung, entsprechende Merkmale zur genaueren Eingrenzung definiert werden.

\subsubsection{Definition der Merkmale}

Zunächst beinhaltet die Destatis-Datenbank nur für Unfälle mit Personenschaden sowie für schwerwiegende Unfälle mit Sachschaden (im engeren Sinne) detaillierte Unfallinformationen, z.B. Unfallursache oder zGG des Fahrzeugs. Bei schwerwiegenden Unfällen mit Sachschaden im engeren Sinne muss als Unfallursache eine Straftat oder Ordnungswidrigkeit im Straßenverkehr bei gleichzeitiger Notwendigkeit ein Kraftfahrzeug aufgrund des Unfallereignisses abzuschleppen. Alle übrigen Sachschadensunfälle, welche ca. 84\% aller polizeilich erfassten Unfälle ausmachen [STA14a], werden nur zahlenmäßig nach Ortslage erfasst und können auch im Rahmen einer detaillierten Sonderabfrage nicht berücksichtigt werden.

Eine erste Einschränkung des in Frage kommenden Fahrzeugspektrums erfolgt durch das Merkmal Schlüsselnummer ,45‘. Die Schlüsselnummer ,45‘ beschreibt „Liefer- und Lastkraftwagen mit Anhänger, die ausschließlich oder hauptsächlich der Beförderung von Gütern dienen“. So lässt sich die Auswertung rein auf Gliederzüge eingrenzen, d.h. Sattelzüge sind nicht Teil des betrachteten Fahrzeugspektrums. Für alle Abfragen gilt das Inlandsprinzip, d.h. es werden alle Unfälle auf deutschem Boden; unabhängig vom Zulassungsland des Fahrzeugs oder dem Herkunftsland des Fahrzeugführers, berücksichtigt. Allerdings werden im Rahmen der Sonderabfrage ausschließlich Unfälle herangezogen, in denen das Güterkraftfahrzeug der Hauptverursacher ist. Ein weiteres entscheidendes Merkmal ist das zGG des 
Fahrzeugs. Dieses wird für ausländische Fahrzeuge nicht erfasst. Hier liegen allein die Verkehrsbeteiligungsarten (z.B. Schlüsselnummer ,45') vor.

Unter den beschriebenen Randbedingungen lassen sich drei Fahrzeugspektren für die Sonderabfrage definieren. Zunächst bilden alle Güterkraftfahrzeuge mit der Schlüsselnummer ,45‘, also alle deutschen und ausländischen Lkw mit Anhänger, das übergeordnete Fahrzeugspektrum. Da eine Auswertung in Bezug auf das zGG nur für deutsche Fahrzeuge möglich ist, bilden alle in Deutschland zugelassenen Güterkraftfahrzeuge mit der Schlüsselnummer ,45‘ die eigentliche Bezugsgruppe zur Identifizierung von Leicht-Lkw im Rahmen der Sonderabfrage. Die Separierung der Leicht-Lkw erfolgt anhand des zGG des Zugfahrzeugs, welches auf 7 bis 7,5 t beschränkt wird, sodass die dritte Gruppe alle in Deutschland zugelassenen Güterkraftfahrzeuge mit Schlüsselnummer ,45' und einem zGG des Zugfahrzeugs von 7 bis 7,5 t umfasst. Allerdings können in dieser Auswahl neben Leicht-Lkw bspw. auch leichte Baustellenfahrzeuge mit kleinem Anhänger enthalten sein. Eine zweifelsfreie Identifikation von 12 t-Leicht-Lkw-Kombinationen ist mit den Destatis-Daten zwar nicht möglich, es kann aber angenommen werden, dass der überwiegende Teil der so separierten Fahrzeugkombinationen tatsächlich den gesuchten Leicht-Lkw-Kombinationen entspricht

\subsubsection{Ortslage}

In Abb. 4-25 wird die Verteilung der Ortslagen der im Zeitraum von 2010 bis 2013 an Unfällen mit Personen- und schwerwiegendem Sachschaden als Hauptverursacher beteiligten deutschen sowie ausländischen Gliederzüge mit der entsprechenden Verteilung für in Deutschland zugelassene Gliederzüge mit zGG des Zugfahrzeugs von 7 bis 7,5 t verglichen.

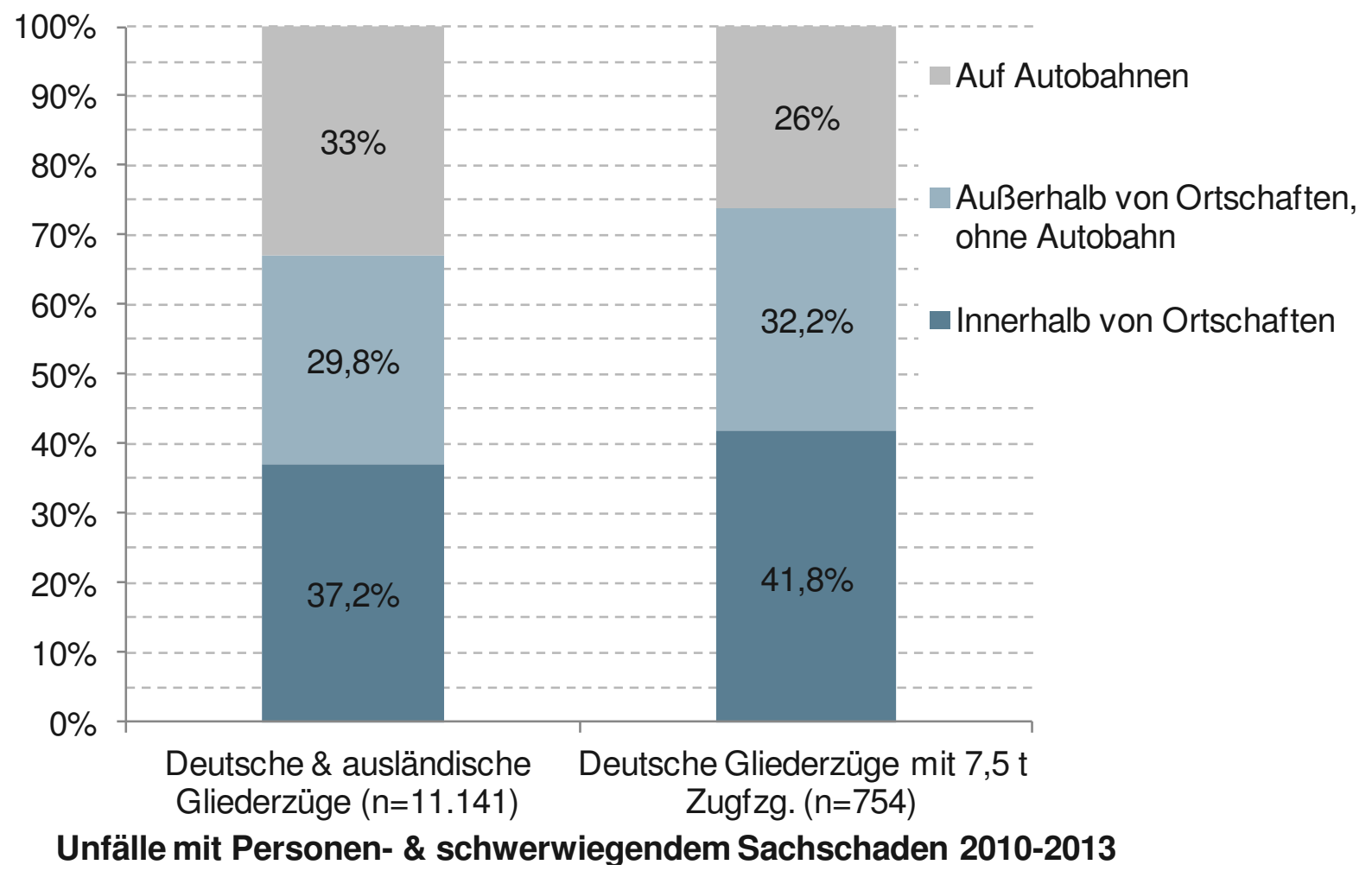

Abb. 4-25: Unfälle mit Personen- \& schwerwiegend. Sachschaden nach Ortslage [STA15a] 
Im Vergleich zu allen beteiligten Lkw mit Anhänger ergibt sich für Leicht-Lkw-Kombinationen mit 42\% ein höherer Anteil innerorts stattfindender Unfälle. Dafür fällt der Autobahnanteil mit $26 \%$ deutlich geringer aus.

\subsubsection{Unfallart}

Hinsichtlich der Unfallart ergeben sich in erster Linie Unterschiede bei den Anteilen von Zusammenstößen mit einem anfahrenden / anhaltenden/ruhenden Fahrzeug sowie bei der Unfallart „Abkommen von der Fahrbahn nach rechts“ (Abb. 4-26). Ersteres hat für die Fahrzeuggruppe der Leicht-Lkw eine deutlich höhere Relevanz während letzteres im Vergleich zu allen an Unfällen mit Personen- und schwerwiegendem Sachschaden als Hauptverursacher beteiligten Gliederzügen seltener vorkommt. Demgegenüber sind die Anteile der übrigen im Rahmen von Destatis ausgewerteten Unfallarten weitestgehend vergleichbar.

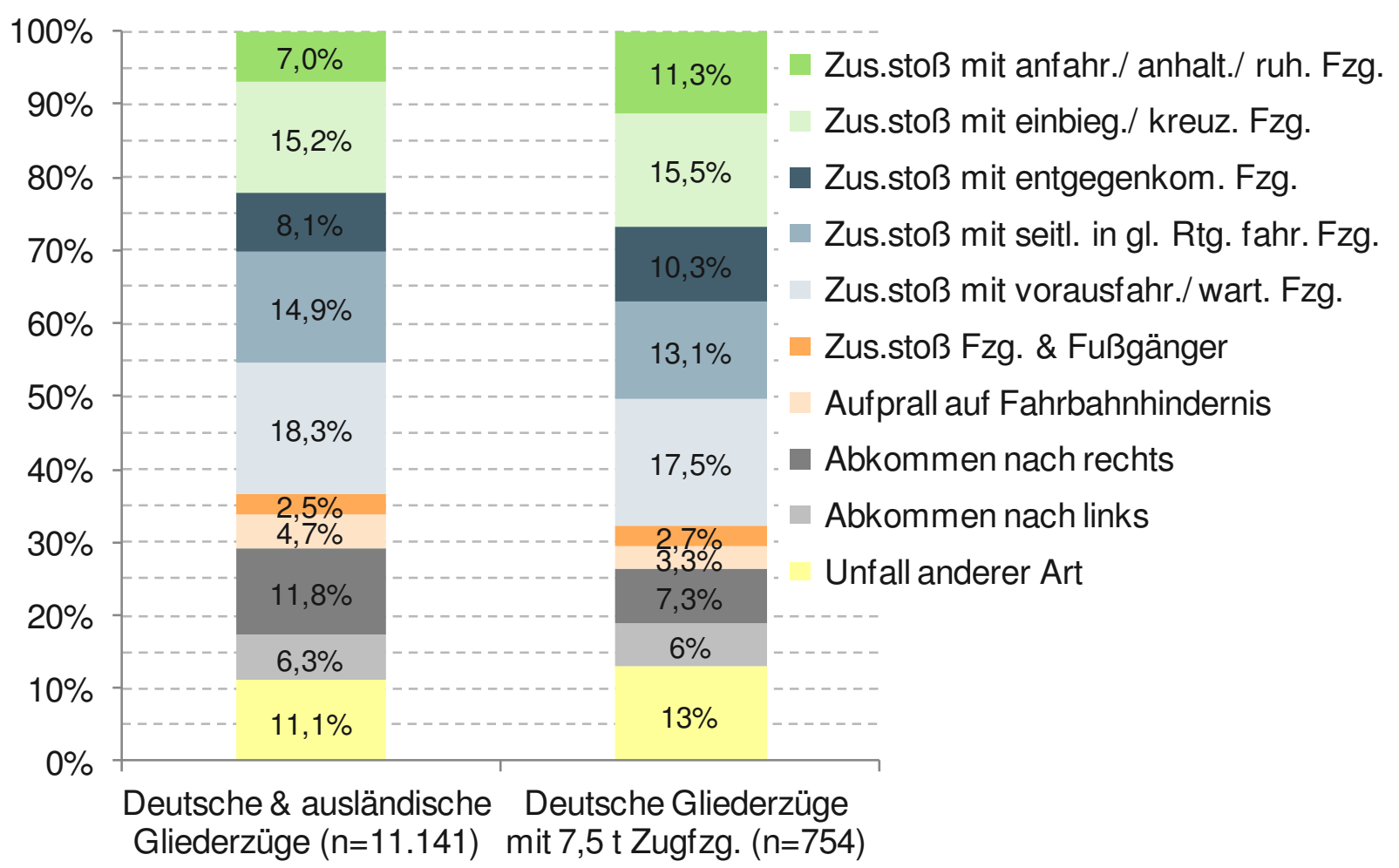

Unfälle mit Personen- \& schwerwiegendem Sachschaden 2010-2013 nach Unfallart

Abb. 4-26: Unfälle mit Personen- \& schwerwiegend. Sachschaden nach Unfallart [STA15a]

\subsubsection{Unfalltyp}

Vergleicht man die Verteilung der verschiedenen Unfalltypen für die beiden betrachteten Fahrzeuggruppen, so ergeben sich keine wesentlichen Unterschiede (Abb. 4-27). Sowohl in Bezug auf alle beteiligten Gliederzüge als auch bei Beschränkung auf die in Deutschland zugelassenen Gliederzüge mit zGG des Zugfahrzeugs zwischen 7 und 7,5 t haben Unfälle im Längsverkehr mit knapp 40\% die höchste Relevanz. Dahinter folgen der Fahrunfall sowie der Einbiegen / Kreuzen-Unfall. 


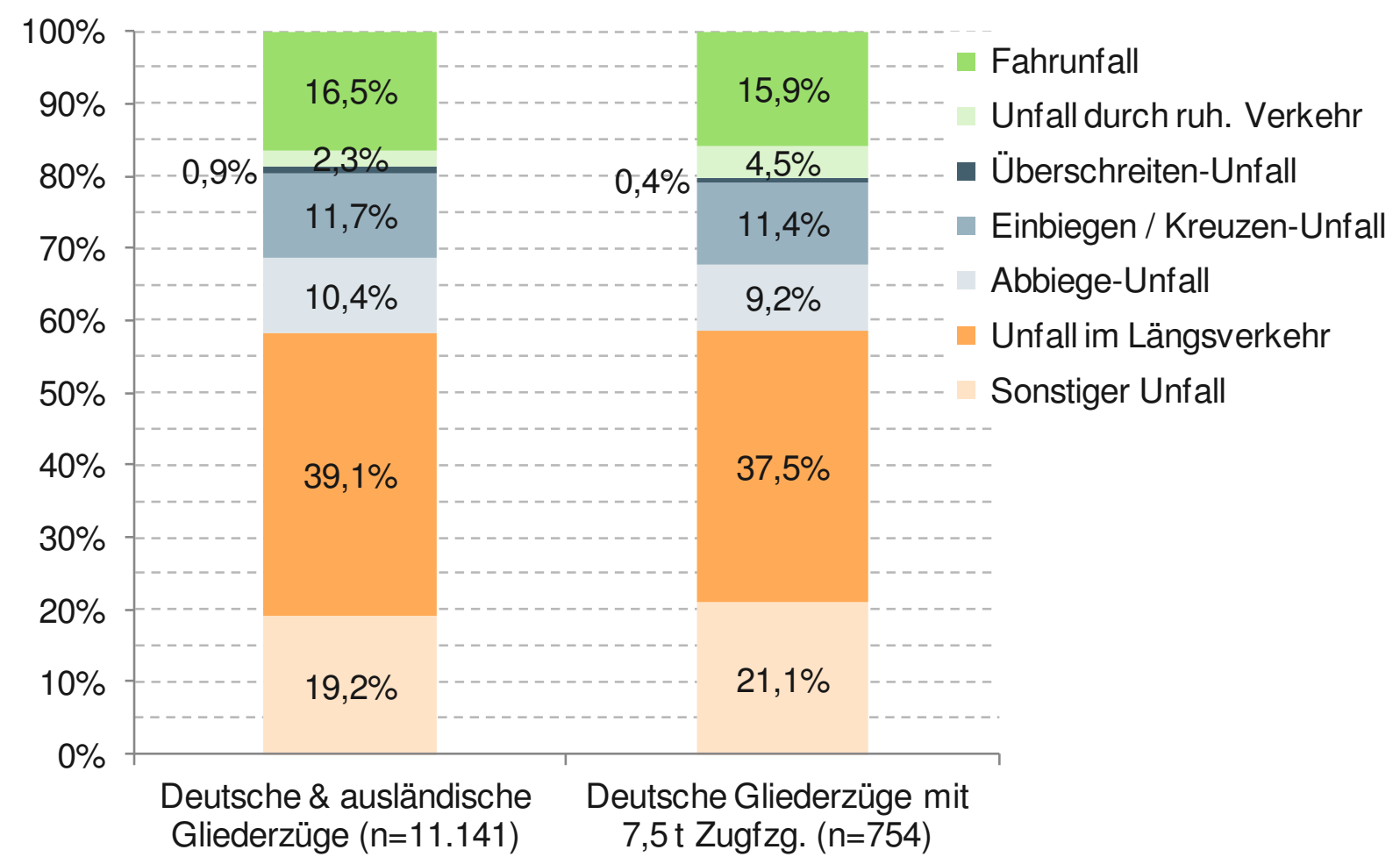

Unfälle mit Personen- und schwerwiegendem Sachschaden 2010-2013 nach Unfalltyp

Abb. 4-27: Unfälle mit Personen- \& schwerwiegend. Sachschaden nach Unfalltyp [STA15a]

\subsubsection{Unfallursachen}

Wie in Kapitel 4.1.2.4 bereits erläutert, wird hinsichtlich der Unfallursachen nach allgemeinen Ursachen, welche dem Unfall und nicht einzelnen Beteiligten zugeordnet sind, sowie personenbezogenen Fehlverhalten unterschieden. Die entsprechenden Verteilungen im Rahmen der Sonderauswertung gehen aus Abb. 4-28 und Abb. 4-29 hervor. Auch wenn ausschließlich Unfälle betrachtet werden, in denen der Lkw-Fahrer der Hauptverursacher ist, werden bedingt durch die Auswertevorgaben auch mögliche Fehlverhalten der Unfallgegner in den Daten enthalten sein.

Betrachtet man zunächst die allgemeinen Unfallursachen, so dominieren Schnee und Eis sowohl bei den Unfällen aller als Hauptverursacher beteiligter Gliederzüge sowie auch in $\mathrm{Be}-$ zug auf in Deutschland zugelassene Gliederzüge mit zGG des Zugfahrzeugs von 7 bis 7,5 t. Auffällig ist für die Fahrzeuggruppe der Leicht-Lkw allerdings der höhere Anteil von Seitenwind als dokumentierte Unfallursache. Dieser beträgt hier über $12 \%$, während er für das übergeordnete Fahrzeugspektrum lediglich bei 3\% liegt. Auch wenn die zugehörige Fallzahl gering ist, spiegeln die Destatis-Daten dennoch die höhere Windanfälligkeit der Leicht-LkwKombinationen wider.

Bezüglich der Fehlverhalten der Unfallbeteiligten zeigen sich keine signifikanten Unterschiede zwischen Gliederzügen allgemein und Gliederzügen mit 7,5 t-Zugfahrzeug. Allerdings fällt der Anteil von Fehlverhalten in Bezug auf Ladung mit 6,4\% bzw. 6,9\% im Vergleich zum Unfallgeschehen von Lkw über 3,5 t zGG (Anteil: 1,1\%, Abb. 4-10) deutlich höher aus. 


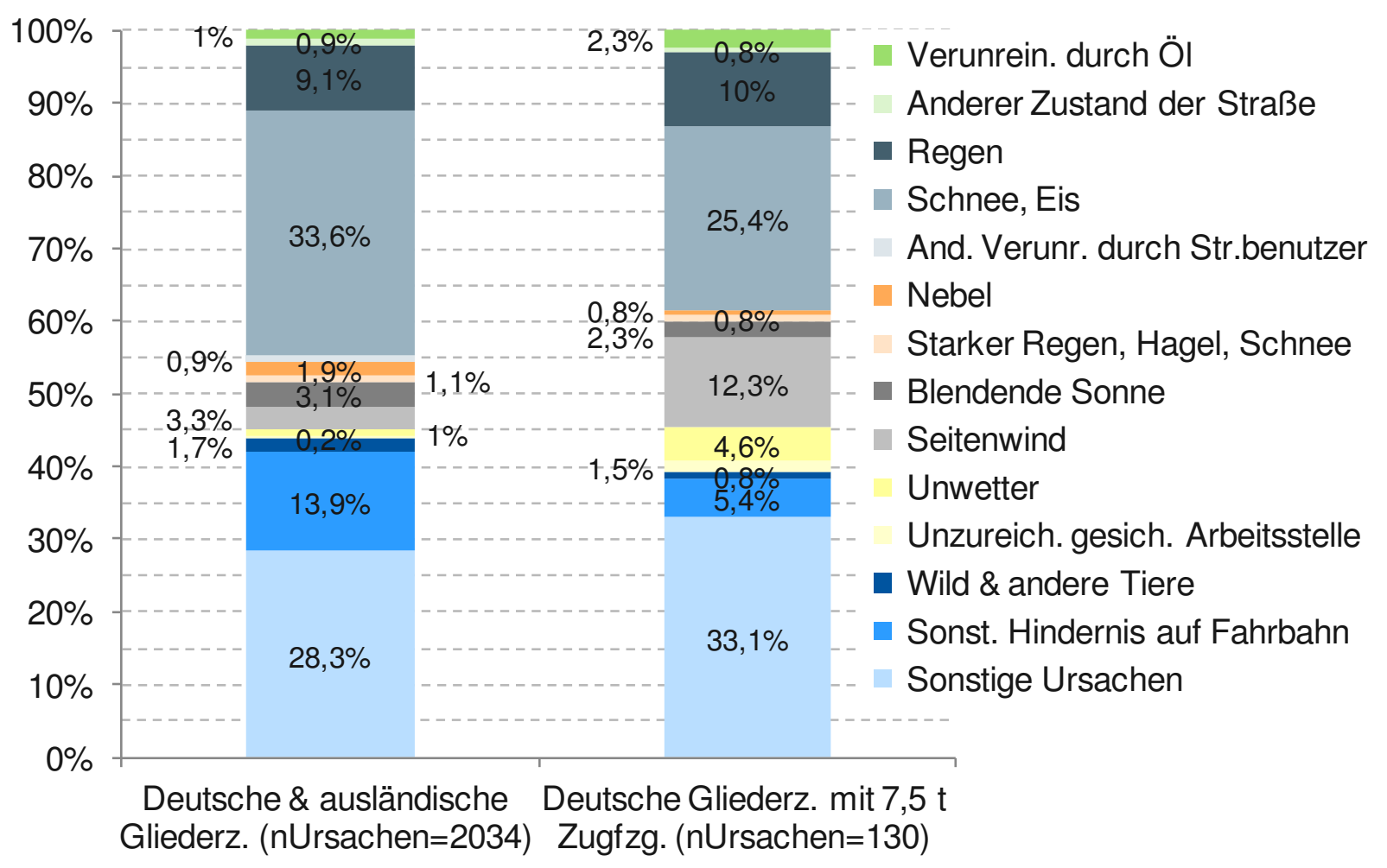

Allgemeine Ursachen bei Unfällen mit Personen- \& schwerem Sachschaden 2010-2013

Abb. 4-28: Unfälle mit Personen- \& schwerwiegendem Sachschaden nach allgemeiner Unfallursache [STA15a]

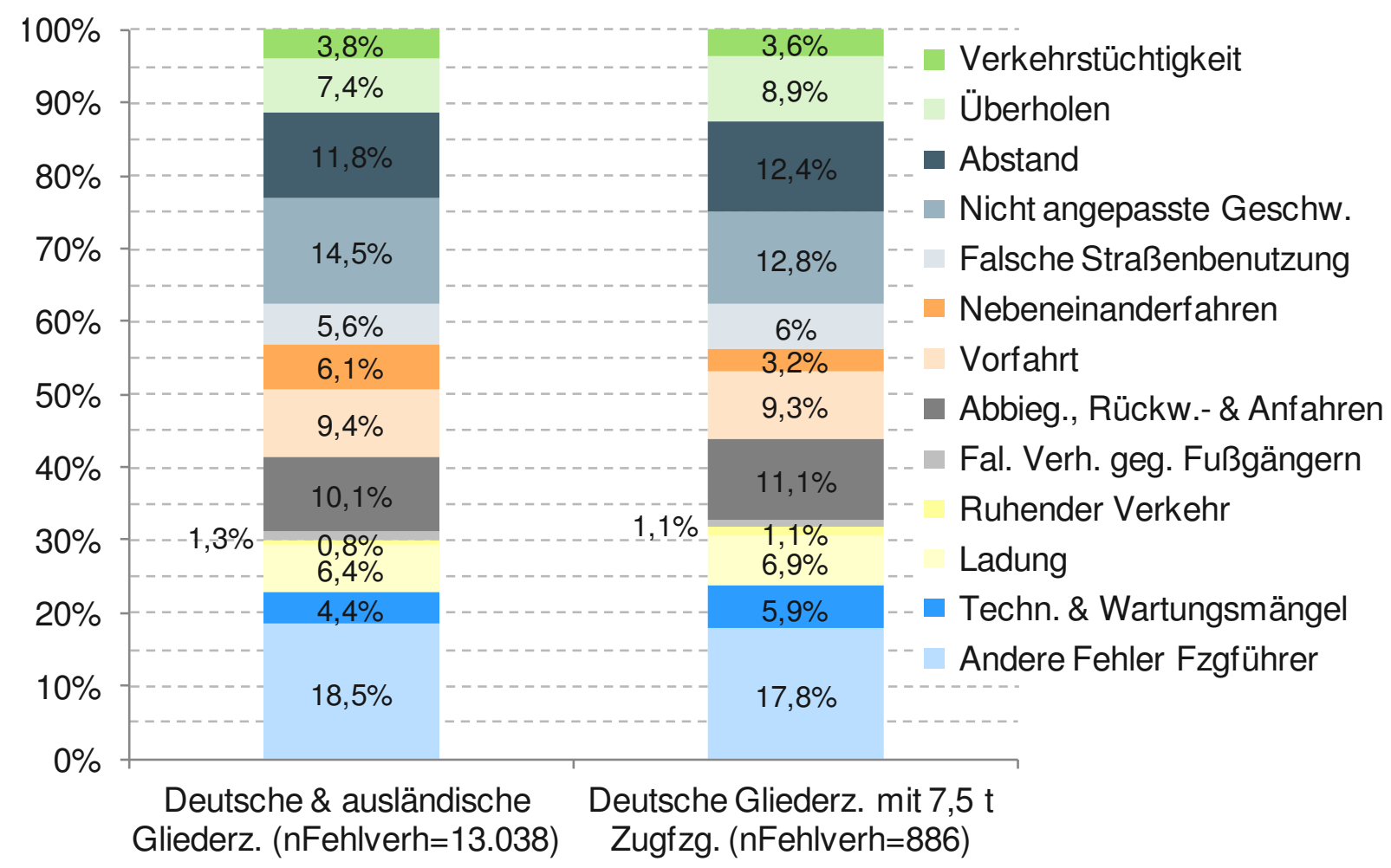

Fehlverhalten der Beteiligten bei Unfällen mit Personen- \& schw. Sachsch. 2010-2013

Abb. 4-29: Unfälle mit Personen- \& schwerwiegendem Sachschaden nach Fehlverhalten als Unfallursache [STA15a] 


\subsubsection{Fahreralter}

Bei den leichten Gliederzügen ergibt sich ein etwas geringerer Anteil der Hauptverursacher mit einem Alter bis 30 Jahren als bei Betrachtung aller in Deutschland im Zeitraum von 2010 bis 2013 verunfallten Gliederzüge (Abb. 4-30).

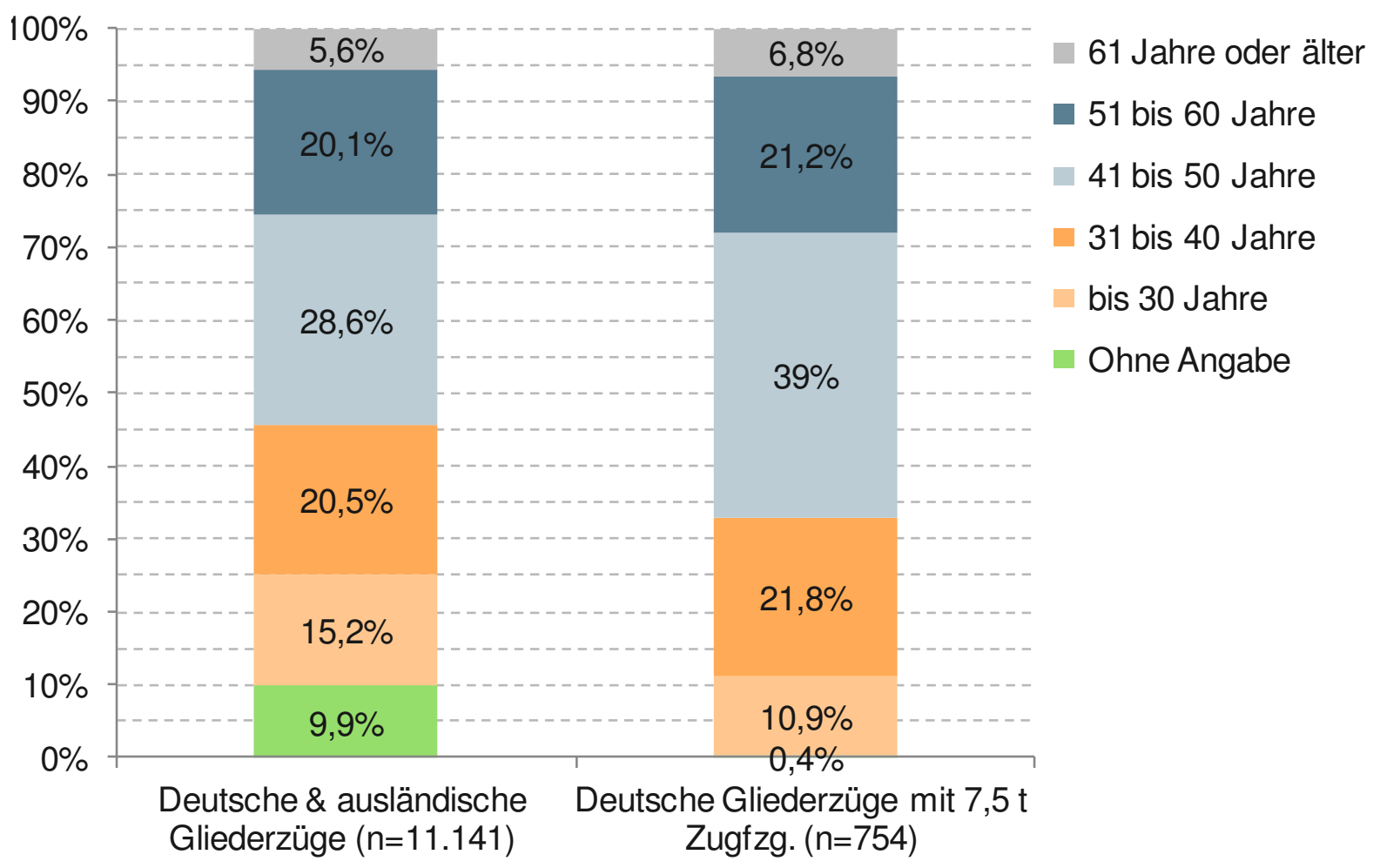

Unfälle mit Personen- \& schwerw. Sachsch. 2010-2013 nach Altersgruppe Hauptverurs.

Abb. 4-30: Unfälle mit Personen- \& schwerwiegendem Sachschaden nach Altersgruppe der Hauptverursacher [STA15a]

Ein naheliegender Grund für diesen Unterschied ist der Umstand, dass nur Fahrer mit altem Pkw-Führerschein (ausgestellt bis 1.1.1999) die $12 \mathrm{t}$-Leicht-Lkw-Kombinationen ohne LkwFührerschein fahren dürfen (Kapitel 2.2), sodass aus wirtschaftlichen Gründen jüngere Fahrer für diese Fahrzeuge weniger in Betracht kommen.

\subsubsection{Aufbau und Auswertung einer Unfalldatenbank}

Die im Rahmen der Sonderauswertung erzielten Fallzahlen für Leicht-Lkw-Unfälle unter Seitenwind sind, gerade mit Hinblick auf die untersuchte Zeitspanne, äußerst gering. Neben der Problematik, dass eine Aufschlüsselung hinsichtlich Leicht-Lkw nur für in Deutschland zugelassene Fahrzeuge möglich ist, wird vermutlich ein großer Teil der Unfälle durch das Statistische Bundesamt nicht in der nötigen Detailtiefe erfasst. Recherchen zu Leicht-LkwUnfällen infolge von Seitenwind zeigen, dass es sich hierbei oftmals weder um Unfälle mit Personenschaden noch um schwerwiegende Unfälle mit Sachschaden, d.h. mit Straftatbestand oder Ordnungswidrigkeit (Bußgeld) und mindestens einem abgeschleppten Kfz, han- 
delt. Zudem finden Unfälle, zu denen die Polizei nicht hinzugezogen wurde, ebenfalls keinen Eingang in die Statistik. Dies könnte der Fall sein, wenn außer dem Unfallverursacher niemand an dem Unfall beteiligt war und die Straße nicht durch den umgestürzten Lkw oder durch verlorene Ladung blockiert ist. Um ein umfassenderes Bild zu Unfällen mit Leicht-Lkw unter Seitenwind zu erhalten, ist im Zuge verschiedener Recherchen, hauptsächlich auf der Basis von Medienberichten, eine Datenbank mit Fällen für die Jahre 2010 bis Mitte 2016 erstellt und ausgewertet worden.

\subsubsection{Ausgewertete Merkmale}

Aus Abb. 4-31 gehen die innerhalb der Unfalldatenbank erfassten Merkmale hervor. Diese können allerdings für die jeweiligen Fälle in der Regel nicht vollständig dokumentiert werden, da die zugehörigen Quellen keine umfassenden Unfallberichte sondern zumeist Nachrichtenmeldungen sind. Entsprechend fehlen einheitliche Standards hinsichtlich der Datenerfassung sowie die notwendige Expertise des Journalisten in Bezug auf Fahrzeugtechnik und Unfallanalyse. Somit können für die in der Datenbank enthaltenen Fälle oftmals nur einzelne Merkmale aufgenommen werden, was teils zu niedrigen Fallzahlen bei der Betrachtung ausgesuchter Merkmale führt. Dies gilt es mit Hinblick auf die Auswertung der Merkmale in Kapitel 4.2.2.2 zu berücksichtigen.

\begin{tabular}{|c|l|}
\hline Merkmal & Beispiel \\
\hline Datum \& Wochentag & \\
\hline Tageszeit & \\
\hline Ortslage \& Infrastrukturmerkmale & z.B. Brücke \\
\hline Witterung & Vorherrschendes Sturmtief, Sturm zum Unfallzeitpunkt \\
\hline Beladungszustand & Ladung, Zugfahrzeug \& Anhänger beladen / unbeladen \\
\hline Geschätzter Sachschaden & \\
\hline Daten zum Fahrer & Alter, Nationalität, Verletzungen \\
\hline Nationalität Fahrzeug & \\
\hline Weitere Personenschäden & \\
\hline Sonstiges & Dauer und Art der Sperrung, Besonderheiten \\
\hline Quelle & Web Link \\
\hline
\end{tabular}

Abb. 4-31: Dokumentierte Merkmale der Unfalldatenbank

Neben den in Abb. 4-31 aufgeführten Merkmalen enthält die Datenbank auch eine Liste der Sturmtiefs in Mitteleuropa ab dem 1. Januar 2010, mit entsprechenden Informationen zu Zeitraum, Sturmtyp, Name und Windgeschwindigkeiten. Über das Datum der dokumentierten Unfälle lässt sich so, sofern nicht bereits im zugehörigen Artikel genannt, ein Bezug zwischen dem jeweiligen Unfall und dem vorherrschendem Sturmtief herstellen. 


\subsubsection{Ergebnisse}

Die Datenbank enthält 149 dokumentierte Leicht-Lkw-Unfälle. Da Informationen bzw. Artikel zu älteren Unfällen oftmals nicht mehr öffentlich zugänglich sind, entfällt ein Großteil der Fälle auf das Jahr 2015, für das die Recherchen zeitnah durchgeführt werden konnten (das Jahr 2016 ist zur Hälfte in der Datenbank berücksichtigt). Zudem kam es hier im Zuge des Sturmtiefs „Niklas“ am 31. März 2015 deutschlandweit zu vielen Leicht-Lkw-Unfällen durch Seitenwind. Von den 88 für das Jahr 2015 erfassten Fällen sind 52 allein auf das Sturmtief „Niklas“ zurückzuführen.

Die Unfälle finden fast ausschließlich tagsüber statt. Es zeigen sich hier in der Regel zwei Szenarien. Oftmals kippt nur der Anhänger und bockt dabei das Zugfahrzeug auf oder aber das gesamte Gespann kippt. Sofern die Unfälle auf Autobahnen stattfinden, verhindern die Leitplanken normalerweise, dass das Zugfahrzeug oder der Anhänger auf die Gegenfahrbahn geraten bzw. abkommen. Eine Auswertung der Datenbank hinsichtlich Ortslage der Unfälle, auftretenden Personenschaden, Beladungszustand des Gespanns sowie geschätzten Sachschaden geht aus Abb. 4-32 hervor.
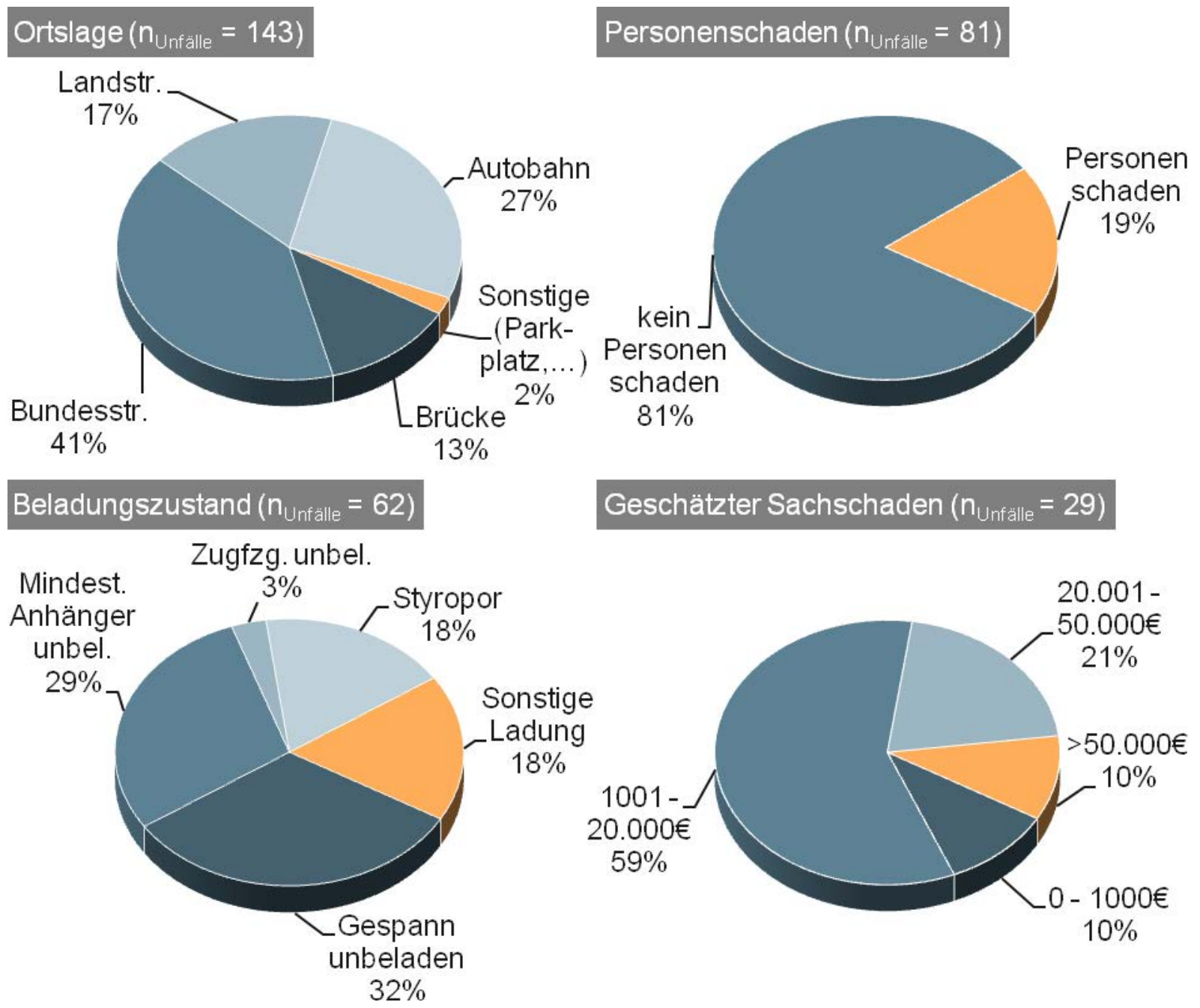

Abb. 4-32: Auswertung verschiedener Merkmale der Unfalldatenbank ( $\mathrm{n}_{\text {Unfälle gesamt }}=149$ ) 
In den Auswertungen sind jeweils nur die Fälle berücksichtigt, für die Angaben zum jeweiligen Merkmal vorliegen. Die Unfälle finden fast ausschließlich außerorts statt, oftmals auf Straßen ohne seitlichen Bewuchs. Dabei machen Brücken einen erheblichen Anteil der Ortslagen aus. Ein charakteristisches Merkmal ist zudem der hohe Anteil (81\%) von Unfällen ohne Personenschaden. Der Lkw-Fahrer ist in der Regel unverletzt oder nur leicht verletzt und kann sich selbstständig aus dem Fahrerhaus befreien. Auch andere Beteiligte werden selten verletzt. Wie oben bereits erwähnt, liegt hierhin vermutlich ein Grund, warum LeichtLkw-Unfälle in der Bundesstatistik unterrepräsentiert sein könnten. Sie werden aufgrund der Unfallrandbedingungen nicht in einer Detailtiefe erfasst, die eine Klassifizierung der Fahrzeuge als 12 t-Leicht-Lkw-Kombination erlaubt.

Kennzeichnend für Leicht-Lkw-Unfälle unter Seitenwind ist auch die Tatsache, dass die Fahrzeuge oft im unbeladenen Zustand verunglücken. Das gilt insbesondere für den Anhänger. In zwei Dritteln der Fälle mit bekanntem Beladungszustand war das gesamte Gespann oder mindestens der Anhänger bzw. das Zugfahrzeug leer unterwegs. In den übrigen Fällen bildet Styropor eine häufige Ladung. Weitere typische Ladungen sind Dämmstoffe, Kunststoffrohre und leere Getränkedosen oder PET-Flaschen. Angaben zum geschätzten Sachschaden werden nur für wenige Fälle gemacht $(n=29)$. Meist liegt dieser unter $50.000 €$. Der höchste Wert beträgt $150.000 €$. Dabei handelt es sich um einen Unfall, wo sowohl am Lastzug als auch an Leitplanken und an einer Brückeneinrichtung Sachschaden entstanden sind.

Die Nationalität der verunfallten Leicht-Lkw-Fahrer und Zugfahrzeuge ist in den meisten Fällen nicht angegeben, sodass die entsprechenden Fallzahlen relativ gering ausfallen. Für die Fahrzeuge kann die Zahl der Fälle mit bekannter Nationalität allerdings durch Auswertung der Unfallbilder nochmals fast verdoppelt werden. Sowohl bei den Fahrern als auch bei den Fahrzeugen ist die Nationalität in fast zwei Dritteln der ausgewerteten Fälle deutsch (Abb. 4-33). Bei den Fahrzeugen ergeben sich zudem relevante Anteile polnischer und tschechischer Fahrzeuge.

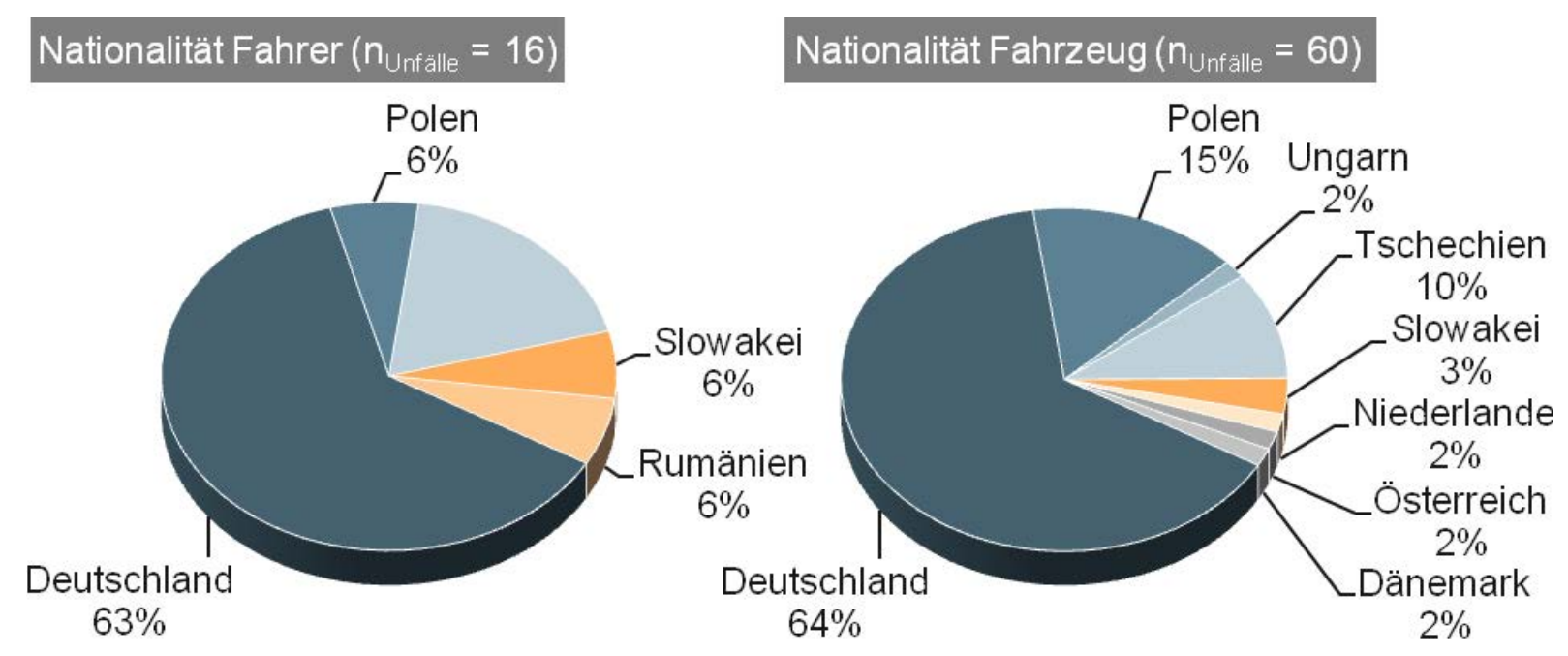

Abb. 4-33: Auswertung Fahrer- \& Fahrzeugnationalitäten 
Im Grunde können alle in der Datenbank enthaltenen Fälle in Zusammenhang mit Sturmtiefs gebracht werden, entweder anhand des Datums oder sogar durch direkte Nennung des Sturmtiefs in der jeweiligen Quelle. Von Interesse sind dabei auch die auftretenden Windgeschwindigkeiten. Maßgeblich ist hier die maximal gemessene Windgeschwindigkeit im Flachland. Orientierung hinsichtlich der damit verbundenen Auswirkungen gibt die Windstärkeskala nach Beaufort, welche in Abb. 4-34 in der Fassung des Deutschen Wetterdienstes (DWD) dargestellt ist. Aufgrund der Tatsache, dass die Windwarnungen des DWD in m/s erfolgen und die Schwellenwerte der Warnungen den Schwellenwerten der m/s-Skala entsprechen, weichen die in der Windwarnskala aufgeführten $\mathrm{km} / \mathrm{h}$ Intervalle geringfügig von der Beaufort-Skala ab [DWD16].

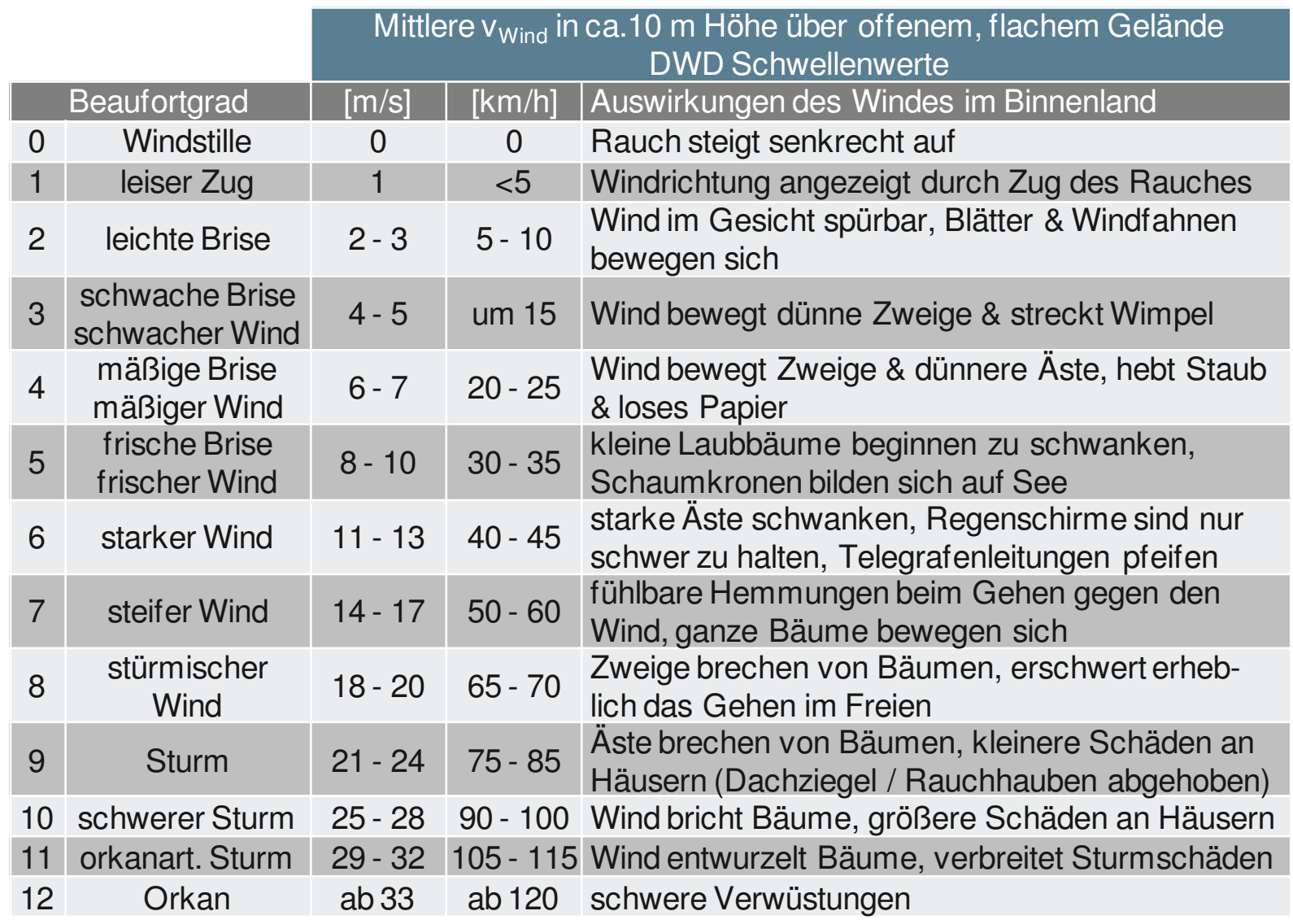

Abb. 4-34: Windwarnskala des DWD in Anlehnung an die Beaufort-Skala [DWD16]

Aus Abb. 4-35 geht hervor, dass die Monate mit hohem Sturmaufkommen den Monaten mit erhöhtem Unfallaufkommen innerhalb der Datenbank entsprechen. Dazu ist auf Basis einer Liste aller Sturmtiefs mit Windgeschwindigkeiten über $75 \mathrm{~km} / \mathrm{h}$ im Flachland die durchschnittliche Zahl der Stürme pro Monat für den betrachteten Zeitraum ermittelt und der jeweiligen Anzahl der Datenbankfälle gegenübergestellt worden. Zu nennen sind in diesem Zusammenhang erwartungsgemäß die Herbst und Wintermonate, vor allem der Zeitraum Dezember bis März. Die durchschnittliche Windgeschwindigkeit liegt hier zwischen 95 und $101 \mathrm{~km} / \mathrm{h}$. Die auffallend hohe Anzahl an Unfällen in der Datenbank, die auf den Monat März entfallen, ist auf die oben bereits erwähnten 52 Fälle infolge des Sturmtief „Niklas“ zurückzuführen. 


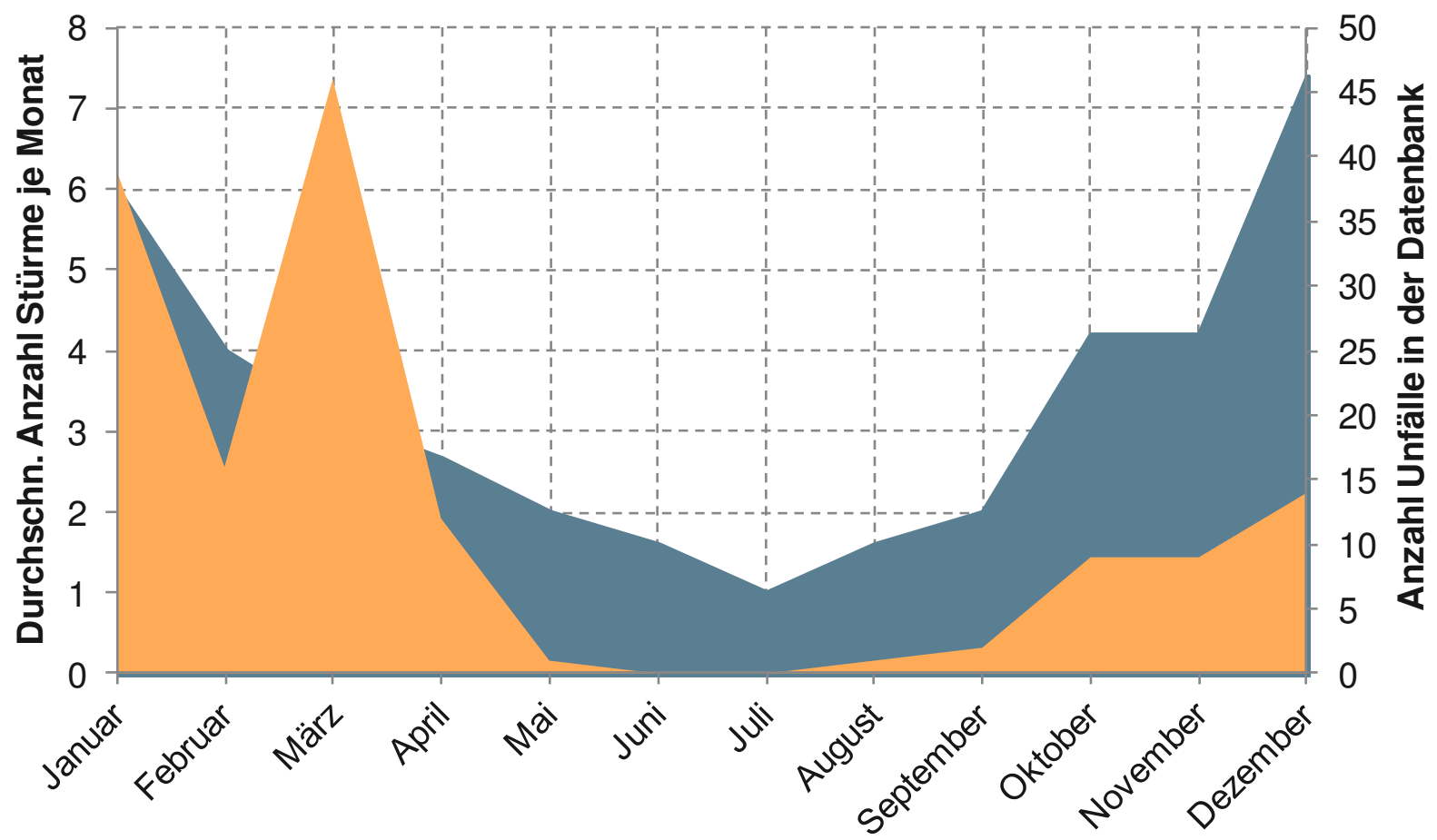

Durchschnittliche Anzahl Stürme ie Monat Anzahl Unfälle in der Datenbank ie Monat

Abb. 4-35: Unfälle in der Datenbank $(n=149)$ und durchschnittliche Anzahl Stürme je Monat

\subsubsection{Auswertung von Schadenakten der Versicherer}

Einen weiteren Einblick in das Unfallgeschehen leichter Gliederzüge geben im Rahmen dieses Projekts zur Verfügung gestellte Schadenakten eines Versicherers. Hierbei handelt es sich überwiegend um Sachschäden ohne Personenschaden (sowohl Haftpflicht- als auch Kaskoschäden). Zudem weist der Großteil der aufgeführten Zugfahrzeuge ein zGG größer 7,5 t auf (Abb. 4-36), sodass der Anteil klassischer 12 t-Leicht-Lkw-Kombinationen im Datenbestand entsprechend gering ausfällt.

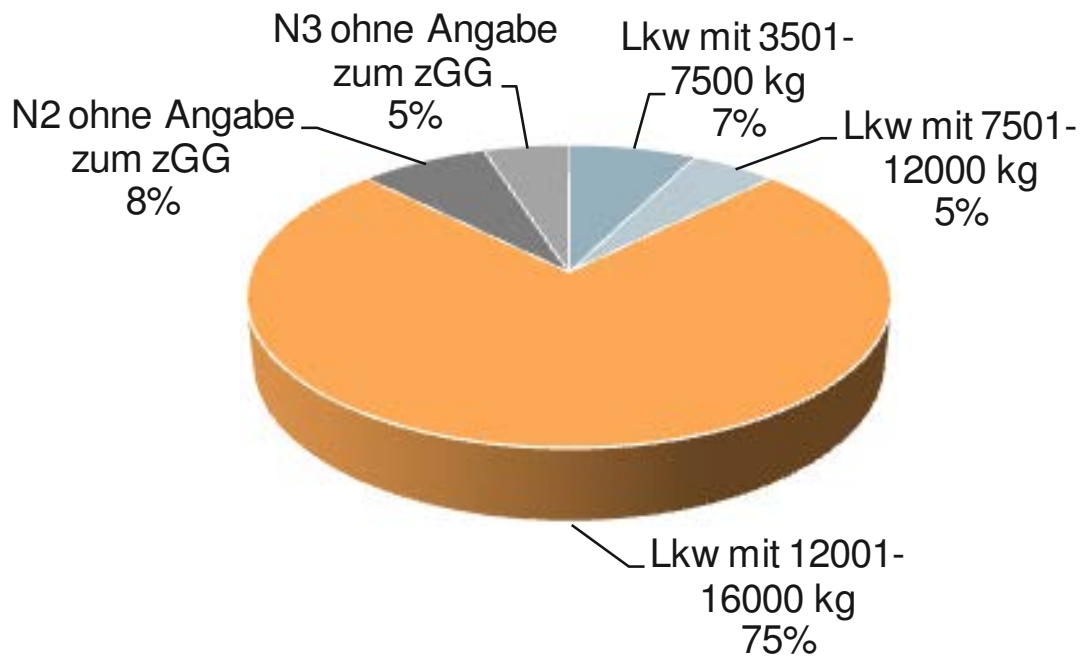

Abb. 4-36: An Unfällen beteiligte Fahrer von Güterkraftfahrzeugen mit zGG größer 3,5 t nach Fahrzeugart $(n=40)$ 
Durch die Vorauswahl der Schadenakten ist dennoch davon auszugehen, dass es sich beim weitaus größten Anteil der verunfallten Lkw in dieser Stichprobe um Volumenzüge handelt, teilweise allerdings mit einem Gesamtzuggewicht von über $12 \mathrm{t}$. Bei letzteren fällt das Zugfahrzeug dann nicht mehr in die 7,5 t-Kategorie, sondern schon in die 12 t-Kategorie. Für die Auswertungen werden allein Schadensfälle mit Gliederzügen berücksichtigt, d.h. die in den Daten enthaltenen Unfälle von Solofahrzeugen werden nicht berücksichtigt. Insgesamt ergibt sich daraus eine Datenbasis von 40 Unfällen.

Aus Abb. 4-37 gehen die Verteilungen der Ortslagen und Straßenklassen hervor. Bezüglich der Ortslagen dominieren die sich innerorts ereignenden Unfälle mit einem Anteil von mehr als drei Vierteln der Datensätze, während die Anteile der außerorts und hier nochmals speziell der auf Autobahnen stattfindenden Unfälle ähnlich sind. Entsprechend hoch fällt mit 44\% der bekannten Fälle der Anteil von Gemeindestraßen aus.

\section{Ortslage $(n=40)$}

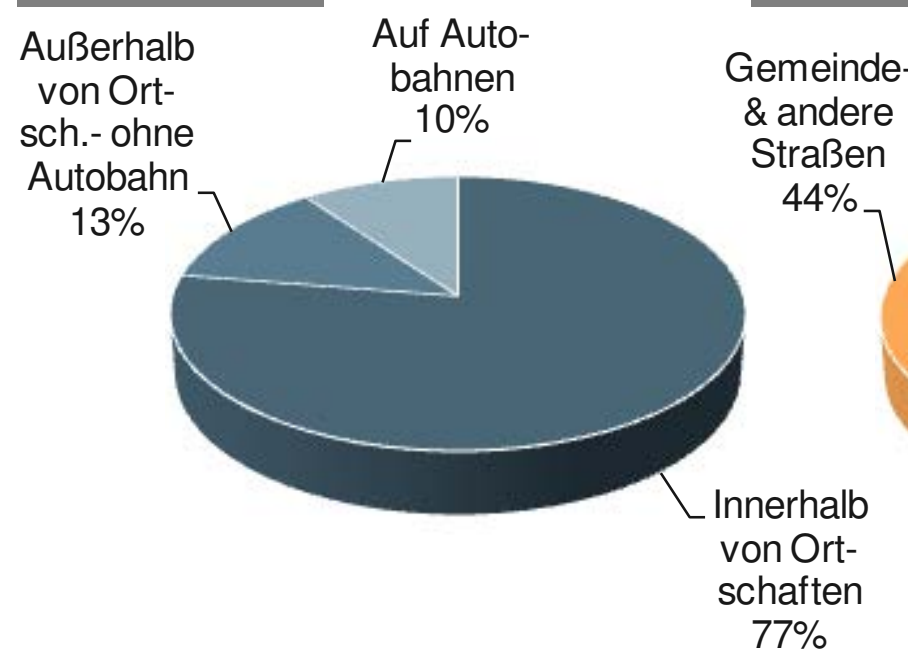

\section{Straßenklasse $(n=34)$}

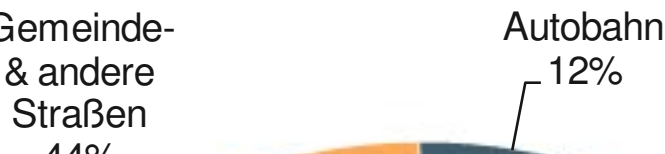

Landesstraße $15 \%$

Abb. 4-37: Unfälle von Güter-Kfz mit zGG größer 3,5 t nach Ortslage \& Straßenklasse

Die Ergebnisse der Auswertungen zu Unfallart, -beteiligten und -typ sowie zum Fehlverhalten der beteiligten Fahrer sind in Abb. 4-38 dargestellt. In mehr als zwei Dritteln der Fälle ist die Unfallart nicht genauer spezifiziert. Die übrigen Fälle weisen einen Zusammenstoß mit einem anderen Fahrzeug aus, wobei Zusammenstöße mit anfahrenden bzw. anhaltenden oder stehenden Fahrzeugen sowie mit entgegenkommenden Fahrzeugen am häufigsten vorkommen. Betrachtet man die Unfallbeteiligten, so ist der hohe Anteil an Alleinunfällen (46\%) auffällig. Dazu zählen aber auch Kollisionen mit Gebäuden oder Einrichtungen am Straßenrand, wie Verkehrszeichen oder Laternen. In etwa einem Drittel der Fälle ist ein Pkw Unfallgegner.

Bezüglich der Fehlverhalten sind besondere Fahrmanöver und zu geringer Abstand auffällig, welche zusammen mehr als drei Viertel der dokumentierten Fehlverhalten ausmachen. Beim übrigen Viertel ist vor allem eine falsche Straßenbenutzung zu nennen, was auf $10 \%$ der Fehlverhalten zutrifft. Der Unfalltyp ist nur für etwa ein Drittel der Unfälle bekannt. Hier 
zeigen sich drei Typen. Am häufigsten ist der Unfall durch ruhenden Verkehr (17\%), gefolgt vom Unfall im Längsverkehr (13\%) sowie dem Fahrunfall (2\%). Die an den Unfällen beteiligten Fahrer von Güterkraftfahrzeugen mit zGG größer 3,5 t sind zu 90\% deutscher Staatsangehörigkeit. Bezüglich der Nationalitätskennzeichen liegt der Anteil deutscher Fahrzeuge sogar bei $95 \%$. Dies ist zum Teil auch dadurch begründet, dass es sich bei den vom Versicherungsunternehmen versicherten Kunden um Transportunternehmer mit Hauptsitz in Deutschland handelt.

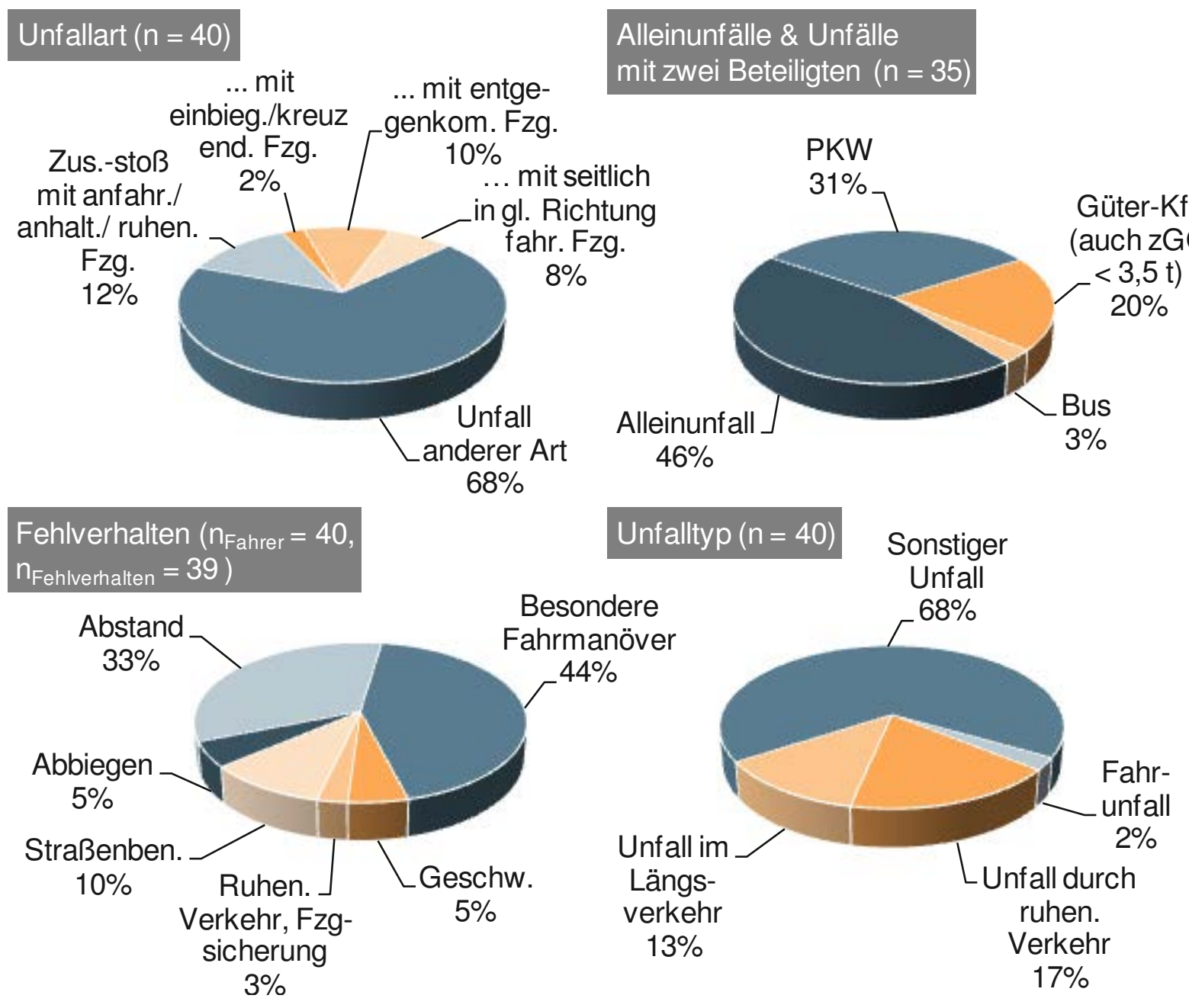

Abb. 4-38: Unfälle von Güterkraftfahrzeugen mit zGG größer 3,5 t nach Unfallart, Unfallbeteiligten, Unfalltyp \& Fehlverhalten der beteiligten Fahrer

Eine allgemeine Unfallursache ist nur für insgesamt vier Unfälle erfasst, wobei in einem Fall Seitenwind angegeben wird. Hierbei handelt es sich um einen durch orkanartige Böen auf der Autobahn hervorgerufenen Unfall mit Leichtverletzten im Herbst 2008. Der Fahrer ist ein 33-jähriger Deutscher. Weitere Informationen konnten bezüglich dieses Falles nicht recherchiert werden. Im Gegensatz zur Auswertung der Unfalldatenbank lassen die in diesem Kapitel vorgestellten Ergebnisse der Schadenaktenanalyse keine Aussagen zum Unfallgeschehen leichter Gliederzüge bei Seitenwind zu, sondern beziehen sich vielmehr auf das allgemeine Unfallgeschehen dieser Fahrzeuge. 


\subsubsection{Fazit}

Insgesamt fallen die im Rahmen der Sonderauswertung erzielten Fallzahlen für Leicht-LkwUnfälle unter Seitenwind, gerade mit Hinblick auf die untersuchte Zeitspanne, äußerst gering aus. Die Auswertungen in Kapitel 4.2.1 und 4.2.2 legen den Rückschluss nahe, dass die amtliche Statistik die absolute Zahl der Unfallereignisse von 12 t-Leicht-Lkw-Kombinationen infolge von Seitenwind unterschätzt. Hierbei spielen mehrere Gründe eine Rolle. Zunächst erfassen die Auswertungen des Statistischen Bundesamtes generell nur Unfälle mit Personenschaden sowie schwerwiegende Unfälle mit Sachschaden im engeren Sinne, d.h. Unfälle bei denen als Unfallursache eine Straftat oder Ordnungswidrigkeit im Straßenverkehr bei gleichzeitigem Abschleppen eines Kraftfahrzeugs vorliegt. Alle übrigen Sachschadenunfälle, welche ca. 84\% aller polizeilich erfassten Unfälle ausmachen [STA14a], werden nur zahlenmäßig nach Ortslage erfasst und können auch im Rahmen einer detaillierten Sonderabfrage nicht näher ausgewertet werden. Die Recherchen zu Leicht-Lkw-Unfällen infolge von Seitenwind im Rahmen der beschriebenen Unfalldatenbank zeigen aber, dass es sich hierbei oftmals weder um Unfälle mit Personenschaden noch um schwerwiegende Unfälle mit Sachschaden handelt. Meist bleibt der Fahrer unverletzt und es liegt auch keine Straftat oder Ordnungswidrigkeit vor. Zudem finden Unfälle, zu denen die Polizei nicht hinzugezogen wurde, ebenfalls keinen Eingang in die Statistik. Dies könnte der Fall sein, wenn außer dem Unfallverursacher niemand an dem Unfall beteiligt war und die Straße nicht durch den umgestürzten Lkw oder durch verlorene Ladung blockiert ist.

Des Weiteren wird das zulässige Gesamtgewicht verunfallter ausländischer Fahrzeuge nicht erfasst. Für diese Fahrzeuge liegen allein die Verkehrsbeteiligungsarten vor. Somit können diese nicht als Leicht-Lkw identifiziert werden und entziehen sich einer entsprechenden Auswertung. Einen möglichen Einfluss wird auch die Unfallaufnahme selbst haben. Die Informationen des Statistischen Bundesamtes basieren auf der Verkehrsunfallanzeige, d.h. Aspekte, wie beispielsweise die Ursache „Seitenwind“, müssen hier auch entsprechend vermerkt sein, um Eingang in die Daten zu finden. Dabei ist die Expertise der Person, die den Unfall aufnimmt, von entscheidender Bedeutung.

Die Auswertung der Unfalldatenbank in Kapitel 4.2.2.2 zeigt, dass im Grunde alle in der Datenbank enthaltenen Fälle in Zusammenhang mit Sturmtiefs gebracht werden können. Die Monate mit hohem Sturmaufkommen entsprechen den Monaten mit erhöhtem Unfallaufkommen in der Datenbank. Zu nennen sind in diesem Zusammenhang erwartungsgemäß die Herbst- und Wintermonate, vor allem der Zeitraum Dezember bis März. Die hier für die Sturmereignisse in den jeweiligen Monaten ermittelten Durchschnittswerte der Windgeschwindigkeit liegen zwischen 95 und $101 \mathrm{~km} / \mathrm{h}$. Die dokumentierten Unfälle finden fast ausschließlich außerorts statt, oftmals auf Straßen ohne seitlichen Bewuchs. Dabei machen Brücken einen erheblichen Anteil der Unfallorte aus. Ein charakteristisches Merkmal ist zudem, wie oben bereits erwähnt, der hohe Anteil (81\%) von Unfällen ohne Personenschaden. Kennzeichnend für Leicht-Lkw-Unfälle unter Seitenwind ist auch die Tatsache, dass die Fahrzeuge oft im unbeladenen Zustand verunglücken. Das gilt insbesondere für den An- 
hänger. In zwei Dritteln der Fälle mit bekanntem Beladungszustand war das gesamte Gespann oder mindestens der Anhänger bzw. das Zugfahrzeug leer unterwegs.

Die im vorangehenden Kapitel vorgestellten Ergebnisse der Analyse von Schadenakten unter Einbeziehung von reinen Sachschadenfällen lassen keine Aussagen zum Unfallgeschehen leichter Gliederzüge bei Seitenwind zu. Derartige Unfallsituationen treten gegenüber Sachschadenunfällen im normalen Fahrbetrieb zahlenmäßig in den Hintergrund. 


\section{$5 \quad$ Betrachtung der Kippstabilität bei Seitenwind mittels rechnerischer Simulation}

Zur simulativen Betrachtung der Kippstabilität von Gliederzügen unter Seitenwind werden drei Vollfahrzeugmodelle in der Mehrkörpersimulationsumgebung Adams/Car aufgebaut. Dabei handelt es sich um zwei Varianten eines 40 t-Gliederzuges, welche als Referenz dienen, sowie um eine $12 \mathrm{t}$-Leicht-Lkw-Kombination.

\subsection{Fahrzeugmodelle}

In einer Mehrkörpersimulation werden starre Körper abgebildet, die über Gelenke oder Kraftelemente gekoppelt werden. Darüber hinaus können äußere Kräfte aufgeprägt werden. Jeder starre Körper wird über eine Punktmasse und die zugehörigen Trägheitsmomente definiert. Ungekoppelt verfügt jeder Körper über sechs Freiheitsgrade, die durch entsprechende Gelenke und Kraftelemente (z.B. Federn) eingeschränkt werden können [ADA14].

\subsection{1 $\quad 40$ t-Gliederzug}

Der Aufbau des Fahrzeugmodells basiert auf einem seitens der fka vorhandenen und validierten Modell eines $40 \mathrm{t}$-Gliederzuges, bestehend aus einem Zugfahrzeug und zwei verschiedenen Anhängertypen. Dabei handelt es sich um einen Gelenkdeichselanhänger sowie um einen Starrdeichselanhänger, welche sich in der Art der Deichsellagerung und in der Anordnung der Achsen unterscheiden. Bei dem in Abb. 5-1 dargestellten 40 t-Gliederzug mit Gelenkdeichselanhänger wird die vordere Achse des Anhängers über einen Drehschemel gelenkt. Der Gelenkdeichselanhänger zeichnet sich durch einen langen Radstand mit kleinen Überhängen aus und wird beispielsweise im Wechselverkehr eingesetzt.

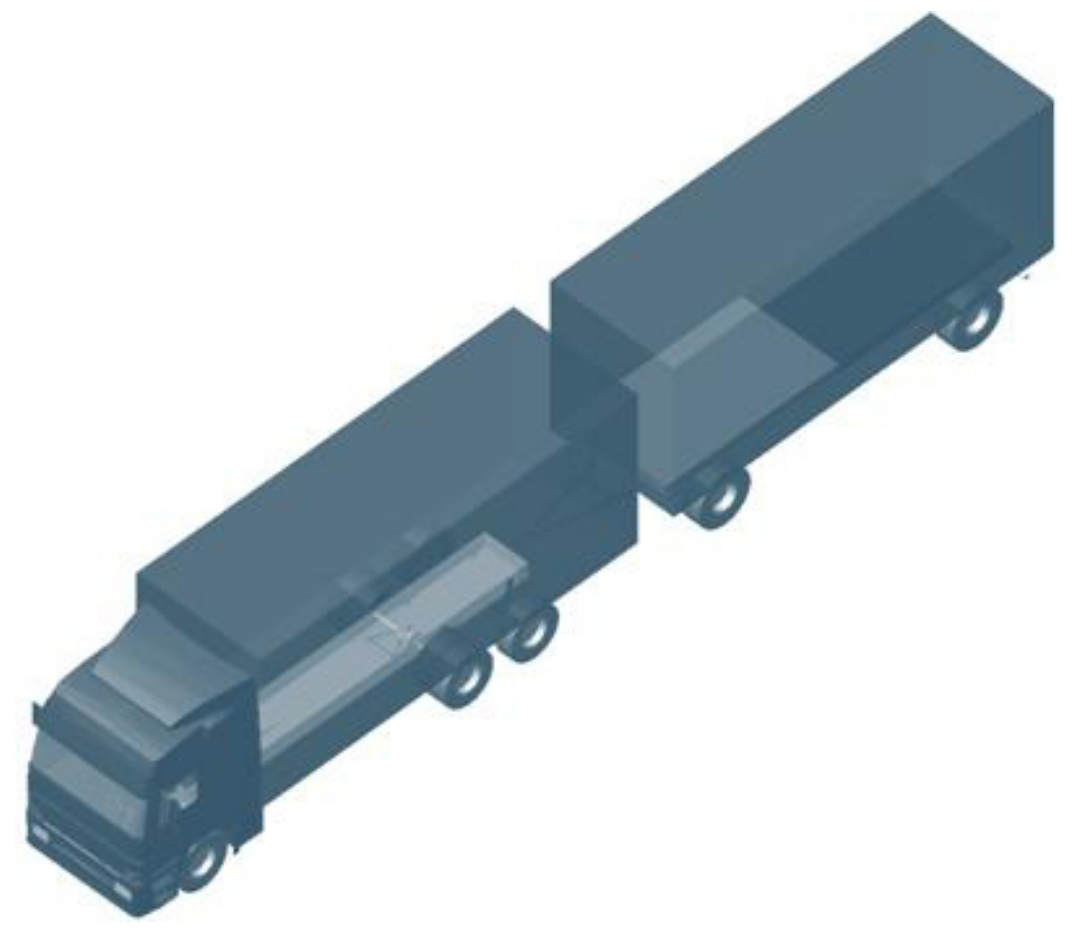

Abb. 5-1: 40 t-Gliederzug mit Gelenkdeichselanhänger 
Beim Starrdeichselanhänger ist die Deichsel starr mit dem Anhängerrahmen verbunden. Er verfügt bei einem $40 \mathrm{t}$-Gliederzug in der Regel über zwei zentrale Achsen und wird daher auch als Zentralachs- bzw. Tandemanhänger bezeichnet. Abb. 5-2 zeigt das Simulationsmodell des 40 t-Gliederzuges mit Starrdeichselanhänger.

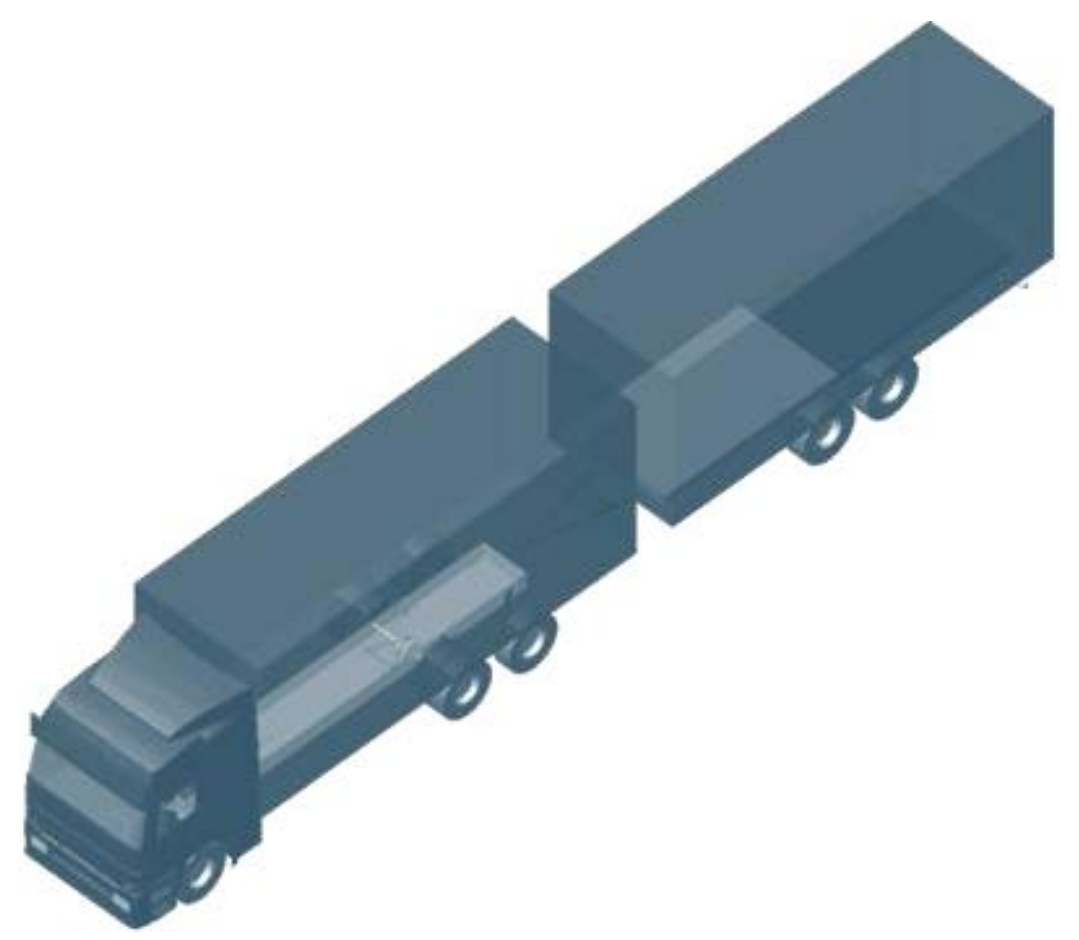

Abb. 5-2: 40 t-Gliederzug mit Starrdeichselanhänger

Für beide Simulationsmodelle gibt Abb. 5-3 jeweils einen Überblick über relevante Fahrzeugparameter, wie Abmessungen, Achslasten und die festgelegten Windangriffsflächen.

\begin{tabular}{|r|c|c|} 
& $\begin{array}{c}40 \text { t Gliederzug mit } \\
\text { Gelenkdeichselanhänger }\end{array}$ & $\begin{array}{c}40 \text { t Gliederzug mit } \\
\text { Starrdeichselanhänger }\end{array}$ \\
\hline Länge $($ Fzg./ Anh.) [m] & $18,75(10,17 / 7,73)$ & $18,75(10,17 / 7,73)$ \\
Höhe [m] & 4 & 4 \\
Breite [m] & 2,55 & 2,55 \\
\hline Windangriffsfläche (Fzg./ Anh.) [m²] & $59,66(33,9 / 25,76)$ & $59,66(33,9 / 25,76)$ \\
\hline Radstand Zugfahrzeug [m] & 6,075 & 6,075 \\
\hline Radstand Anhänger [m] & 5,2 & 1,3 \\
\hline Felgengröße [“] & 22,5 & 22,5 \\
\hline Federung Fahrzeug & Blatt / Luft & Blatt / Luft \\
\hline Federung Anhänger & Luft & Luft \\
\hline Achslasten Fzg. (beladen) [t] & $7,0 / 9,0 / 5,8$ & $7,0 / 9,5 / 6,3$ \\
\hline Achslasten Anhänger (bel.) [t] & $8,7 / 9,2$ & $8,7 / 8,5$ \\
\hline Leergewicht (Fzg./Anh.) [t] & $14,7(10,2 / 4,5)$ & $15,1(10,2 / 4,9)$ \\
\hline Zuladung (Fzg./ Anh.) [t] & $25,3(11,8 / 13,5)$ & $24,9(11,8 / 13,1)$ \\
\hline
\end{tabular}

Abb. 5-3: Abmessungen und Parameter der 40 t-Fahrzeugmodelle 


\subsubsection{2 t-Gliederzug}

Für den $12 \mathrm{t}$-Gliederzug kann auf kein bestehendes Simulationsmodell zurückgegriffen werden. Die Grundlage für den Modellaufbau bildet die Vermessung eines realen Gespanns. Die hierbei gewonnenen Daten dienen der Parametrierung des Fahrzeugmodells.

\subsubsection{Vermessung eines realen Fahrzeugs}

Neben den allgemeinen Fahrzeugparametern wird im Zuge der Vermessung auch die Schwerpunkthöhe des Anhängers bestimmt. Die zu diesem Zweck gemietete Leicht-LkwKombination ist in Abb. 5-4 dargestellt.

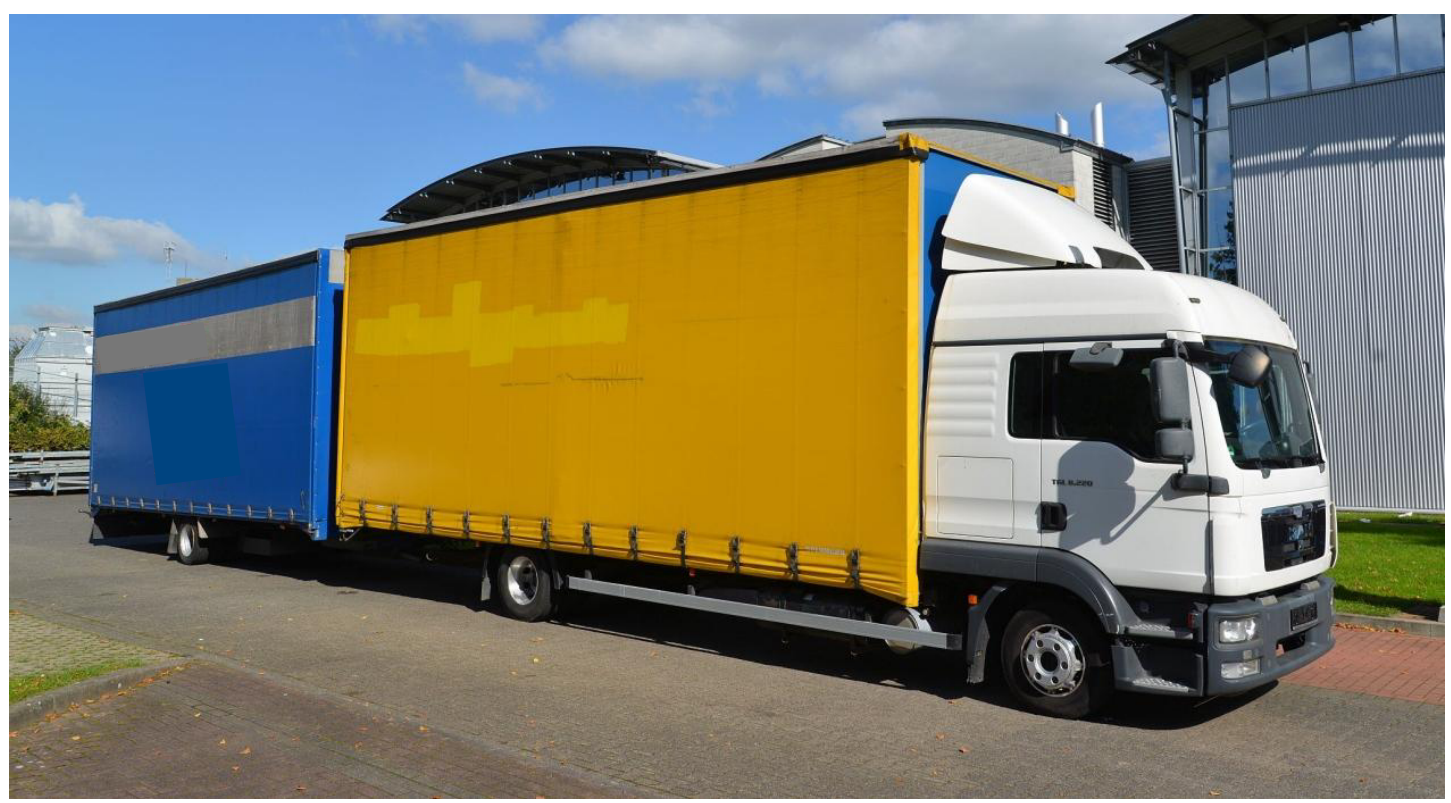

Abb. 5-4: Im Rahmen des Projekts vermessene Leicht-Lkw-Kombination

Das zulässige Gesamtgewicht des einachsigen Starrdeichselanhängers beträgt 4500 kg, wobei die Messungen zur Bestimmung der Schwerpunkthöhe ausschließlich am leeren Anhänger durchgeführt werden. Zur Definition von links- bzw. rechtsseitiger Bereifung wird die Fahrtrichtung als Bezugsrichtung gewählt. Zur Ermittlung der Schwerpunkthöhe kommen insgesamt drei Radlastwaagen mit einer Genauigkeit von +/- 0,2 kg zum Einsatz. Diese werden jeweils rechts und links unter der Bereifung der Achse sowie unter die Deichselabstützung positioniert. Die Radlasten werden dabei in drei Konfigurationen aufgenommen:

1) Anhänger in waagerechter Lage

2) Anhänger nach links gekippt

3) Anhänger nach rechts gekippt

Das Kippen des Anhängers wird durch Unterlegen einer Europalette auf der jeweiligen Achsseite realisiert, wie in Abb. 5-5 dargestellt. Dies entspricht einer definierten Anhebung um insgesamt 0,144 m. 


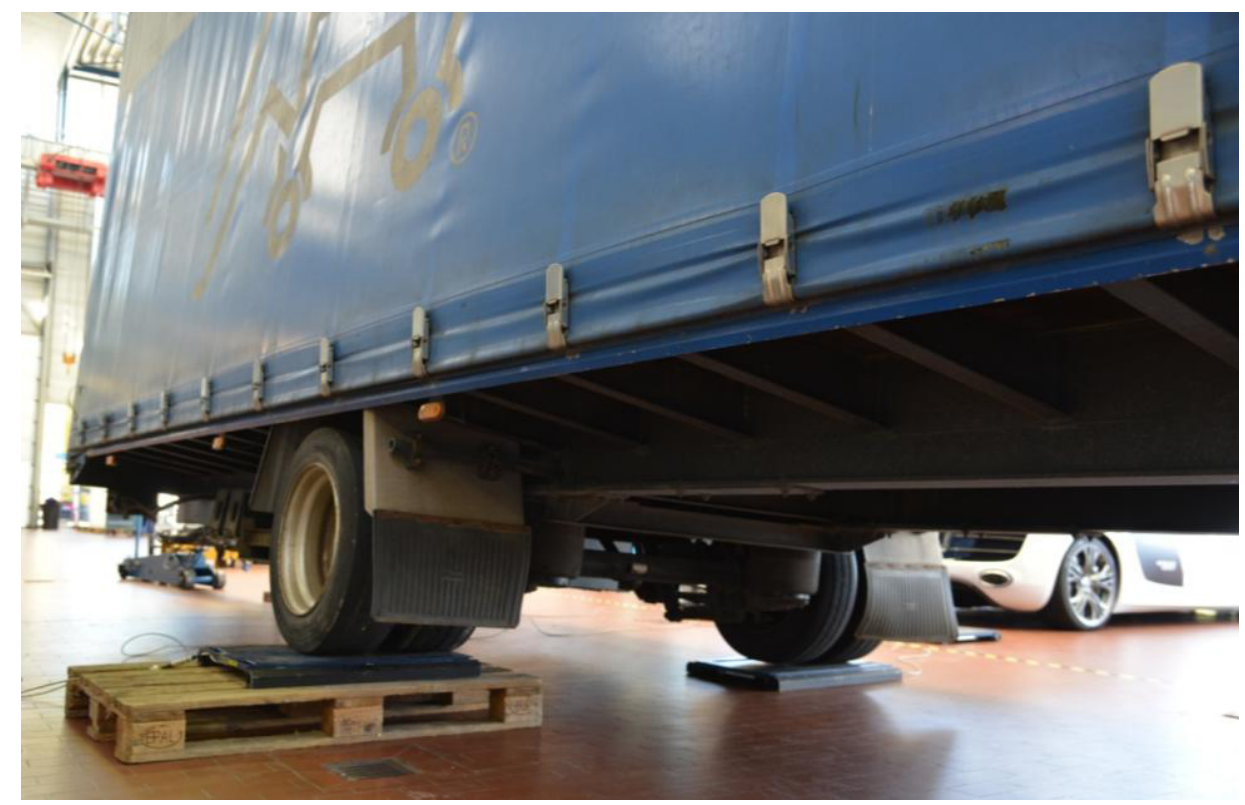

Abb. 5-5: Radlastkonfiguration 3 - Anhänger nach rechts gekippt (Ansicht von hinten)

Die Ergebnisse der Radlastmessungen gehen aus Abb. 5-6 hervor. Durch das Kippen der starren Achse ergibt sich eine Verschiebung des Radaufstandspunktes (vgl. Abb. 5-7), die bei der späteren Berechnung der Schwerpunkthöhe berücksichtigt werden muss.

\begin{tabular}{|l|c|c|c|c|}
\hline \multicolumn{1}{|c|}{ Konfiguration } & Last rechts [kg] & Last links [kg] & Last Deichsel [kg] & Gesamt [kg] \\
\hline 1 Waagerecht & 991,6 & 920,4 & 250,2 & 2162,2 \\
\hline 2 Nach links gekippt & 950 & 954,2 & 255,8 & 2160 \\
\hline 3 Nach rechts gekippt & 1000 & 894,2 & 262 & 2156,2 \\
\hline
\end{tabular}

Abb. 5-6: Ergebnisse Radlastmessungen

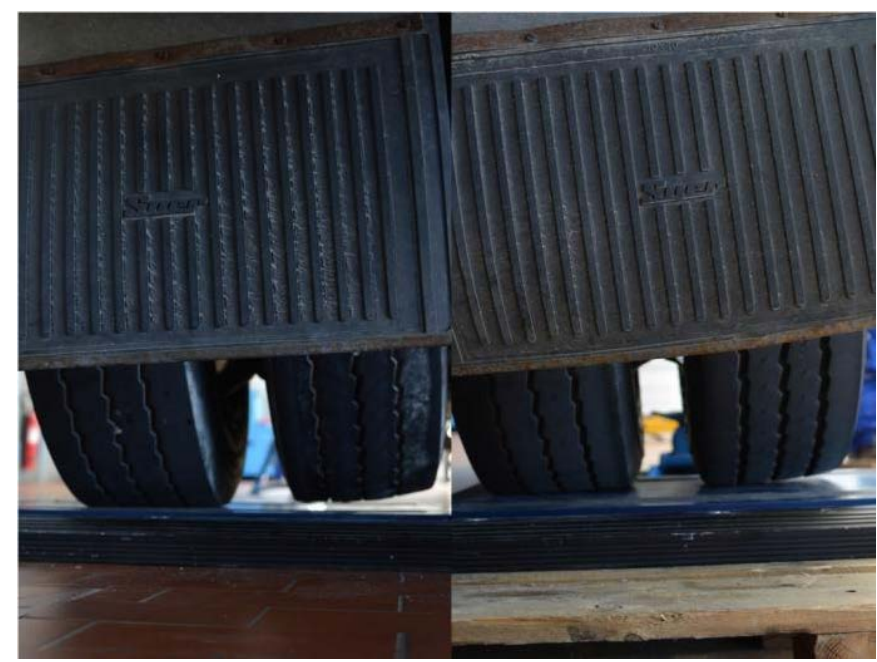

Abb. 5-7: Verschiebung des Radaufstandspunktes links - rechts (hier: Konfiguration 2) 
Die Ergebnisse der Anhängervermessung sind in Abb. 5-8 dargestellt. Die Messergebnisse werden im folgenden Kapitel zur Berechnung der Anhängerschwerpunkthöhe sowie zur Parametrierung des Modells verwendet. Des Weiteren wird zur Absicherung der Ergebnisse eine Sensitivitätsanalyse durchgeführt, bei der anhand realistisch angenommener Messungenauigkeiten die Güte der Messung bewertet wird.

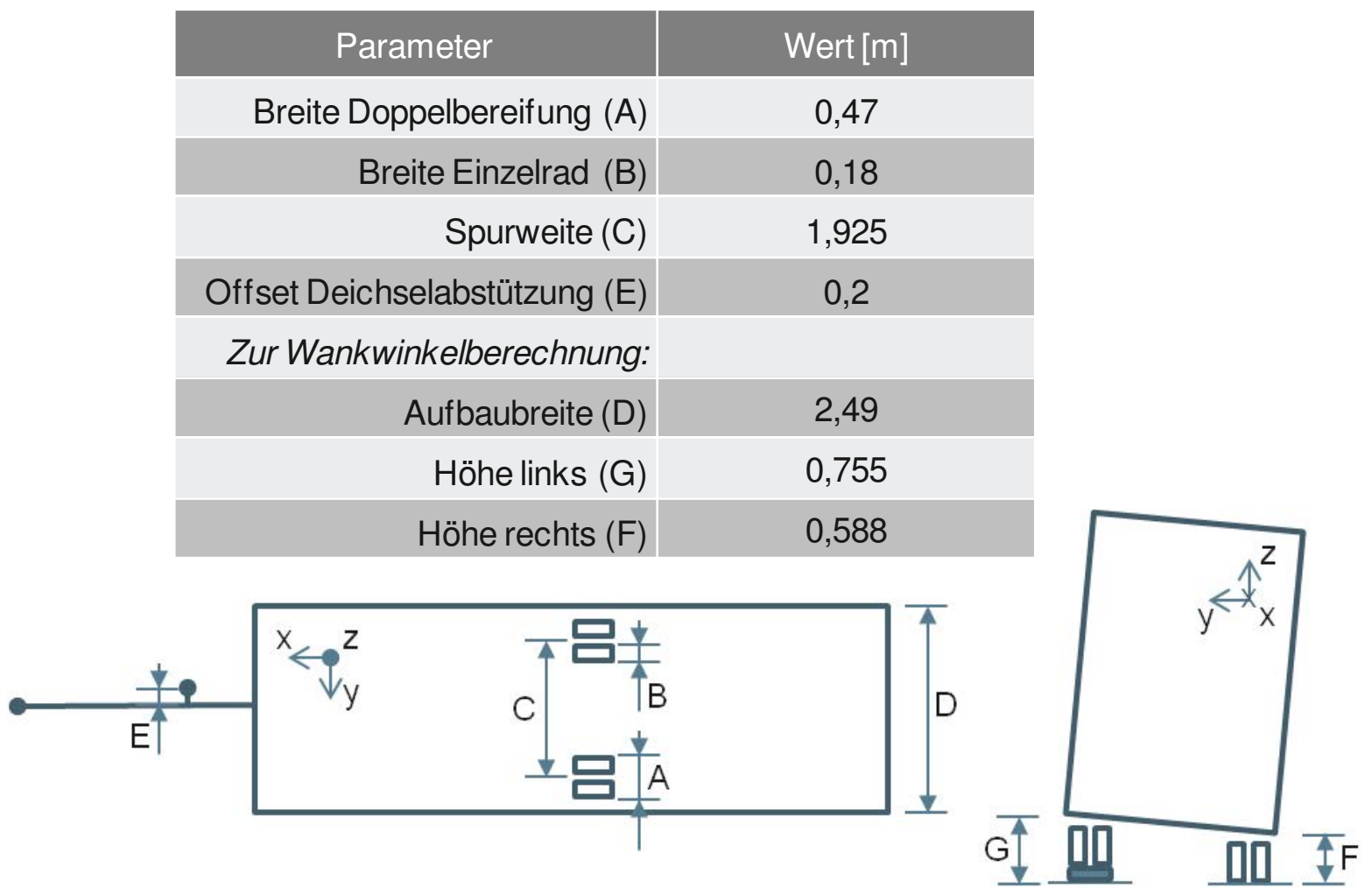

Abb. 5-8: Geometrische Messgrößen in der Draufsicht (Konfiguration 1) und Ansicht von hinten (Konfiguration 3)

\subsubsection{Aufbau des Fahrzeugmodells}

Da die Messung der Radlasten in der Ausgangslage des Anhängers bereits eine hohe Asymmetrie aufweist (Abb. 5-6), wird hier zunächst die Schwerpunktlage in y-Richtung ausgehend von der linken Bereifung errechnet.

Der Abstand des Schwerpunktes zur linken Bereifung ergibt sich über ein Kräftegleichgewicht aus den gemessenen Radlasten (F), der Spurweite (C) und dem Versatz der Deichselabstützung (E) gemäß Gl. 5-1.

$$
H=\frac{\mathrm{F}_{\text {Deichsel }} *\left(\frac{C}{2}+E\right)+\mathrm{F}_{\text {Rechts }} * C}{\mathrm{~F}_{\text {Deichsel }}+\mathrm{F}_{\text {Rechts }}+\mathrm{F}_{\text {Links }}}=1,0173 \mathrm{~m}
$$

Des Weiteren ergibt sich aus den beidseitigen Höhen der unteren Aufbaukante im gekippten Zustand (G \& F, vgl. Abb. 5-8) und der Aufbaubreite (D) der Wankwinkel Ф nach Gl. 5-2. 


$$
\Phi=\left(\arcsin \frac{G-F}{D}\right) * \frac{180}{\pi}=3,8456^{\circ}
$$

Die hervorgerufene Schrägstellung der Achse führt bereits zu einer deutlichen Verschiebung der Radaufstandspunkte (Abb. 5-7). Im Folgenden wird angenommen, dass sich an der angehobenen Anhängerseite der Radaufstandspunkt der Doppelbereifung zur Radmitte des inneren Rades verschiebt, während er sich auf der nicht angehobenen Seite zur Radmitte des äußeren Rades verschiebt. Die angenommene Verschiebung ergibt sich mit den in Abb. 5-8 aufgeführten Werten aus Gl. 5-3.

$$
K=\frac{A-B}{2}=0,145 m
$$

Die Schwerpunktlage ergibt sich schließlich aus Gl. 5-4 und Gl. 5-5. Zur Verifikation werden jeweils beide Momentengleichgewichte, d.h. für den linken sowie den rechten Radaufstandspunkt, betrachtet.

$S P_{L}=\frac{\left(\mathrm{F}_{\text {Deichsel }} *\left(\frac{C}{2}+E-K\right)+\mathrm{F}_{\text {Rechts }} * C-\left(\mathrm{F}_{\text {Deichsel }}+\mathrm{F}_{\text {Rechts }}+\mathrm{F}_{\text {Links }}\right) *(H-K)\right.}{\left(\mathrm{F}_{\text {Deichsel }}+\mathrm{F}_{\text {Rechts }}+\mathrm{F}_{\text {Links }}\right) * \sin \Phi} \quad$ Gl. 5-4
$S P_{R}=\frac{\left(\mathrm{F}_{\text {Deichsel }} *\left(\frac{C}{2}-E-K\right)+\mathrm{F}_{\text {Rechts }} * C-\left(\mathrm{F}_{\text {Deichsel }}+\mathrm{F}_{\text {Rechts }}+\mathrm{F}_{\text {Links }}\right) *\left(\frac{C}{2}-H-K\right)\right.}{\left(\mathrm{F}_{\text {Deichsel }}+\mathrm{F}_{\text {Rechts }}+\mathrm{F}_{\text {Links }}\right) * \sin \Phi} \quad$ Gl. 5-5

Der aus GI. 5-4 und GI. 5-5 berechnete Mittelwert ergibt eine Schwerpunkthöhe (SP) des Anhängers von 1,48 m. Dieses Ergebnis wird im Zuge einer Sensitivitätsanalyse weiter abgesichert. Dabei werden die Werte der gemessenen Radlasten im Bereich der Messgenauigkeit der verwendeten Waagen, d.h. um +/- 0,2 kg, und die aufgenommenen geometrischen Messgrößen (Abb. 5-8) um jeweils $+/-5 \mathrm{~mm}$ variiert.

Die Sensitivitätsanalyse zeigt, dass der Einfluss möglicher Radlastschwankungen aufgrund einer Messungenauigkeit der verwendeten Waagen in Bezug auf die Schwerpunkthöhe mit einem Streuband von 0,002 m zu vernachlässigen ist. Demgegenüber kann die Kombination fehlerbehafteter geometrischer Messgrößen zu einer Streuung der Schwerpunkthöhe um +/$0,15 \mathrm{~m}$ führen. Den größten Einfluss hat hierbei die gemessene Reifenbreite, die allerdings als bekannter Parameter vorausgesetzt und damit als Fehlerquelle ausgeschlossen werden kann. Zur Validierung durchgeführte Simulationen zeigen, dass im angenommenen Streuband eine Schwerpunkthöhenvariation vernachlässigbaren Einfluss auf die Fahrdynamik bzw. die Kippstabilität hat und der beschriebene Ansatz somit in das Fahrzeugmodell integriert werden kann.

Analog zum vermessenen Gespann verfügt das Simulationsmodell der 12 t-Leicht-Lkw-Kombination über einen einachsigen Starrdeichselanhänger (Abb. 5-9). Der Modellaufbau erfolgt durch Skalierung des Fahrzeugmodells des $40 \mathrm{t}$-Gliederzuges auf Basis der zuvor erhobenen Daten. Dabei wird die Fahrerhausgeometrie eines 7,5 t-Zugfahrzeugs implementiert. Abb. 5-10 gibt einen Überblick über die relevanten Modellparameter des 12 t-Gliederzuges. 


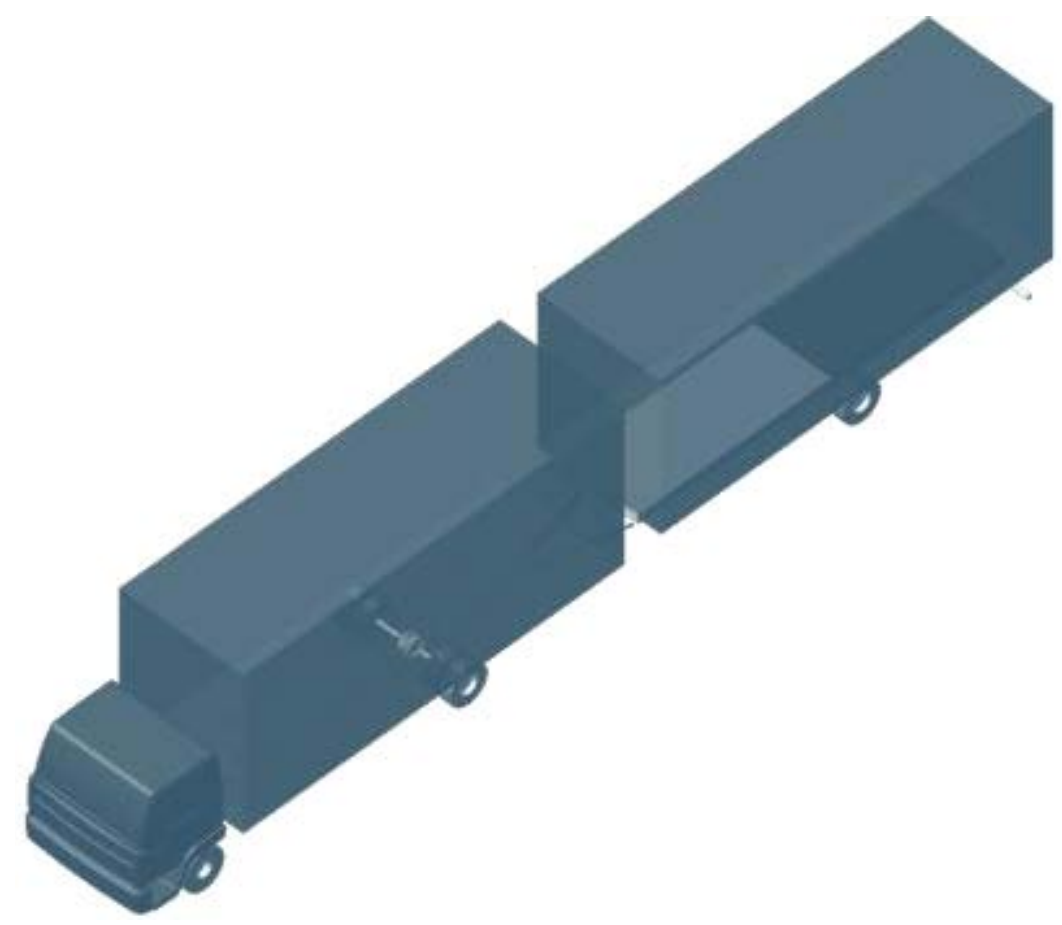

Abb. 5-9: 12 t-Gliederzug mit Starrdeichselanhänger

\begin{tabular}{|r|c|} 
& $\begin{array}{c}12 \text { t Gliederzug mit } \\
\text { Starrdeichselanhänger }\end{array}$ \\
\hline Länge (Fzg./ Anh.) [m] & $18,75(10,17 / 7,82)$ \\
Höhe [m] & 4 \\
Breite [m] & 2,55 \\
\hline Windangriffsfläche (Fzg./Anh.) [m²] & $59,9(33,9 / 26)$ \\
\hline Radstand Zugfahrzeug [m] & 5,4 \\
\hline Radstand Anhänger [m] & 6,4 (Kupplung bis Achsmitte) \\
\hline Felgengröße [“] & 17,5 \\
\hline Federung Fahrzeug & Blatt / Luft \\
\hline Federung Anhänger & Luft \\
\hline Achslasten Zugfahrzeug (beladen) [t] & $4,2 / 3,6$ \\
\hline Achslasten Anhänger (beladen) [t] & 4,2 \\
\hline Leergewicht (Fzg./ Anh.) [t] & $7,2(5,0 / 2,2)$ \\
\hline Zuladung (Fzg./ Anh.) [t] & $4,7(2,5 / 2,2)$ \\
\hline
\end{tabular}

Abb. 5-10: Abmessungen und Parameter des 12 t-Fahrzeugmodells

\section{$5.2 \quad$ Seitenwindmodell}

Zur Untersuchung der Kippstabilität der Fahrzeugmodelle unter Seitenwind ist die Definition und Implementierung eines entsprechenden Seitenwindmodells erforderlich. Dies umfasst in einem ersten Schritt die Modellierung der Windkraft. Die Widerstandskraft, die auf einen Körper in einer Gasströmung wirkt, wird durch Gl. 5-6 beschrieben. 
Mit

$$
F_{S W}=\frac{1}{2} * \rho * A * v_{W i n d}^{2} * c_{w}
$$

$\rho \quad$ Luftdichte

A Windangriffsfläche

$v_{\text {Wind }}$ Windgeschwindigkeit

$c_{w} \quad$ Luftwiderstandsbeiwert in Querrichtung

Dabei sind die Luftdichte $\rho$, die Windangriffsfläche $\mathrm{A}$ und die Windgeschwindigkeit $v_{\text {Wind }}$ direkt bestimmbar. Die Luftdichte $\rho$ beträgt bei $20^{\circ} \mathrm{C} 1,2 \mathrm{~kg} / \mathrm{m}^{3}$ und wird über alle Simulationen als konstant angenommen. Die Windangriffsfläche $A$ ist definiert als das Produkt der Aufbauhöhe und der Aufbaulänge der im Wind stehenden Flächen. Die Flächen der dem Wind ausgesetzten Achsen sind gegenüber den Flächen der Aufbauten vernachlässigbar gering. Die Windgeschwindigkeit $v_{\text {Wind }}$ geht quadratisch in die Formel des Luftwiderstandes ein und stellt einen variablen Parameter für die Simulationen zur Kippstabilität dar.

Der Luftwiderstandsbeiwert $c_{w}$ ist stark von der Aerodynamik des Fahrzeugs und seiner Umgebung abhängig und wird im Windkanal bestimmt. Da 40 t-Gliederzüge aufgrund der hohen Masse nur wenig Seitenwindempfindlichkeit aufweisen, sind folglich kaum Veröffentlichungen zu Versuchen unter reiner Queranströmung bekannt. Darüber hinaus muss eine möglichst gleichmäßige, unverwirbelte Strömung gewährleistet werden, was den Einsatz von Modellen oder entsprechend großen Windkanaldurchmessern erfordert.

Im Zuge der fehlenden Referenzwerte müssen für die Definition des Seitenwindmodells sinnvolle Annahmen bezüglich des Luftwiderstandsbeiwerts in Querrichtung getroffen werden. Laut [FRA13] liegen die Luftwiderstandsbeiwerte für Gliederzüge bei einer Schräganströmung von $6^{\circ}$ zur Fahrzeuglängsachse bereits bei Werten von bis zu 1,3. Auf Basis anderer Quellen, in denen grundlegende Untersuchungen für stumpfe Körper und Schienenfahrzeuge durchgeführt werden, wie [HUC11] und [ALA07], lässt sich eine realistische Eingrenzung des Luftwiderstandsbeiwerts auf Werte zwischen 1,5 und 2 vornehmen. In [ALA07] werden Modellversuche für Schienenfahrzeuge im Windkanal durchgeführt. Die Ergebnisse sind in Abb. 5-11 dargestellt. Bei einem Windangriffswinkel von $90^{\circ}$ quer zur Fahrtrichtung liegt der Luftwiderstandsbeiwert bei 1,8. In [BLY08] wird der Einfluss des Luftwiderstandsbeiwertes bezogen auf die Seitenfläche eines Sattelzuges untersucht. Die berücksichtigten Werte liegen dabei zwischen 1 und 2. Der Vergleich mit Ergebnissen von Baker aus dem Jahre 1991 liefert ebenfalls eine Eingrenzung auf einen Wertebereich von 1,5 bis 2. Aufgrund fehlender aerodynamischer Daten innerhalb der Untersuchung wird in [BLY08] eine konservative Abschätzung des Beiwertes nach oben empfohlen, d.h. es sollte ein Wert von etwa 2 verwendet werden.

In [CHE11] werden u.a. auch Gliederzüge bezüglich des Luftwiderstandsbeiwerts in Querrichtung betrachtet. Ein Vergleich mit einem Solo-Lkw auf einer flachen Fahrbahn in Abhängigkeit des Anströmwinkels zeigt ab einem Winkel von $60^{\circ}$ etwas höhere Werte für einen Gliederzug (Abb. 5-12). Für beide Fahrzeuge steigt der Luftwiderstandsbeiwert dabei bis zur reinen Queranströmung, d.h. $90^{\circ}$ zur Fahrtrichtung des Fahrzeugs, kontinuierlich an. 


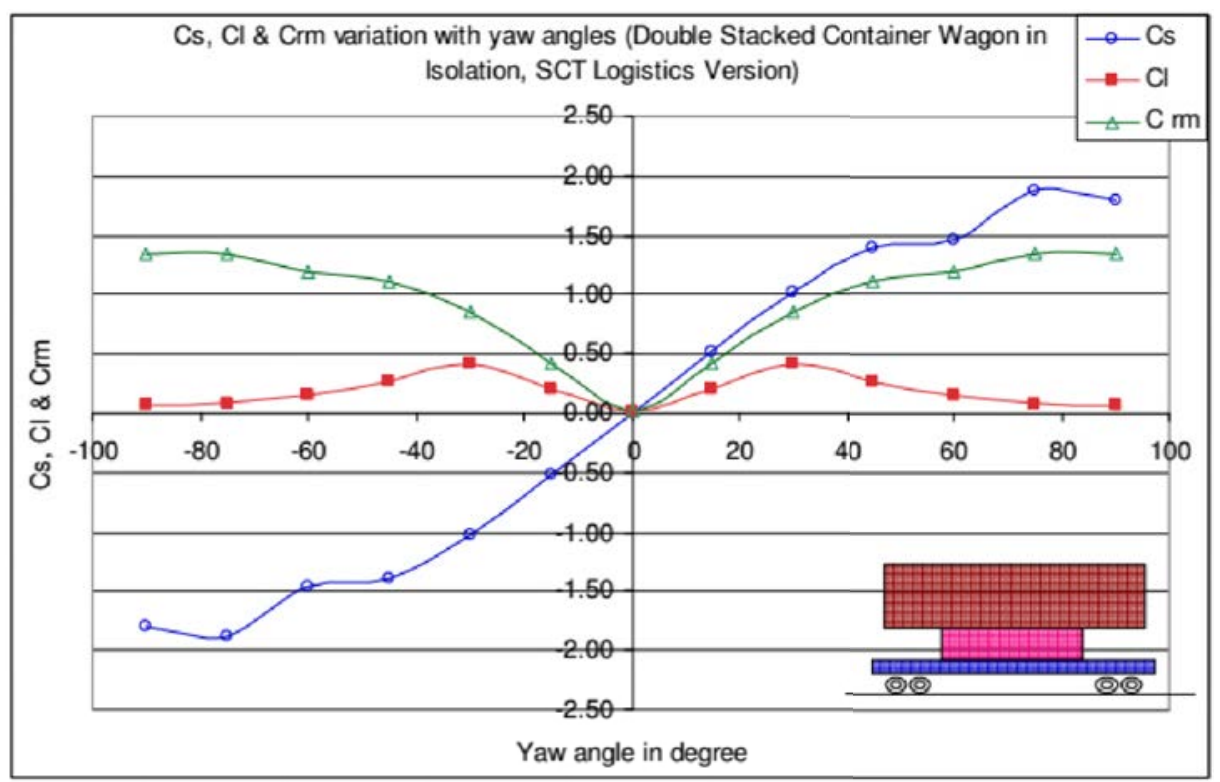

Abb. 5-11: Aerodynamische Luftwiderstandsbeiwerte in Querrichtung (blau) nach [ALA07]

Die in Abb. 5-12 dargestellte Abhängigkeit des Luftwiderstandsbeiwerts in Querrichtung vom seitlichen Anströmwinkel legt nahe, dass ein $90^{\circ}$ zur Fahrtrichtung angreifender Wind als kritischster Fall in Bezug auf die Kippstabilität eines Gliederzuges zu sehen ist, da der Luftwiderstandsbeiwert und damit auch die sich daraus ergebende seitliche Windkraft (GI. 5-6) hier ihren Maximalwert erreichen. Entsprechend wird für das Seitenwindmodell eine $90^{\circ}$ zur Fahrtrichtung angreifende Windkraft definiert.

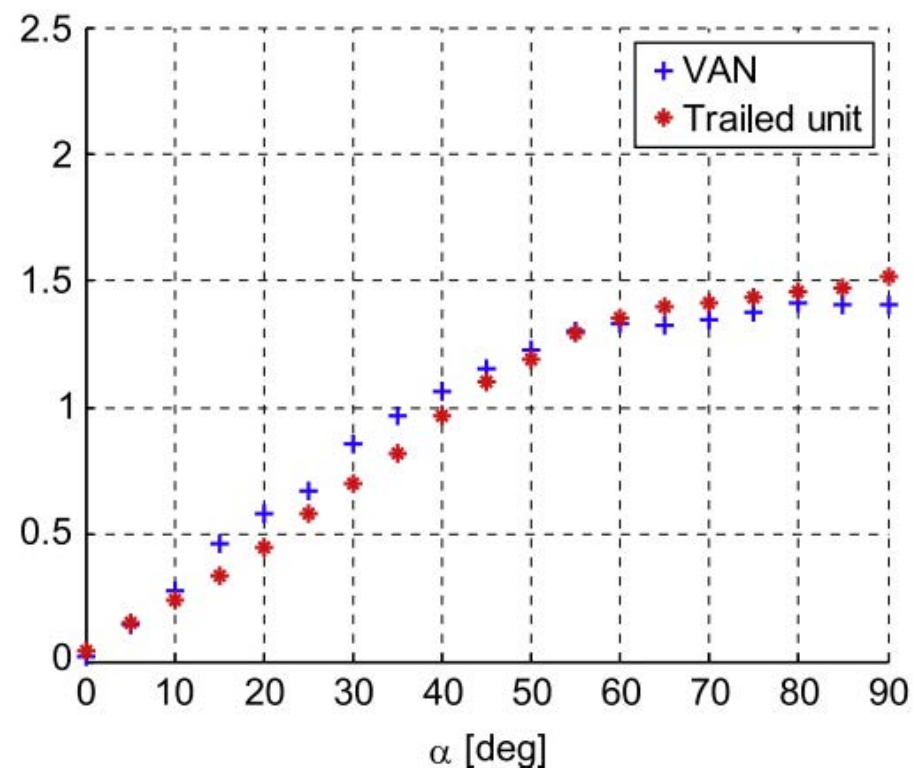

Abb. 5-12: Luftwiderstandsbeiwert in Querrichtung über Anströmwinkel für einen Solo-Lkw (blau) und einen Gliederzug (rot) [CHE11]

Der Luftwiderstandsbeiwert in Querrichtung stellt die einzige aerodynamische Einflussgröße im Rahmen des Seitenwindmodells dar. Mögliche weitere aerodynamische Einflüsse, bei- 
spielsweise aufgrund der Längsbewegung des Fahrzeugs, können in der Mehrkörpersimulationsumgebung nicht abgebildet werden. Somit ist die Definition des Luftwiderstandsbeiwerts maßgeblich für die in der Simulation auftretende Windlast. In [CHE11] werden für einen Lkw ohne Anhänger verschiedene Fahrsituationen unter Seitenwind betrachtet. Dabei handelt es sich, neben einer Fahrt auf einer flachen Straße, um die Überfahrt eines Dammes, einer Talbrücke sowie einer Doppelbrücke, die im Windkanal nachempfunden werden. In Abb. 5-13 ist zu erkennen, dass sich Luftwiderstandsbeiwerte zwischen 1,4 (flache Straße) und 2,1 (Überfahren einer Talbrücke) ergeben. Dazwischen liegen die Werte für eine Doppelbrücke bei 1,7 und für das Überfahren eines Dammes bei 1,8.

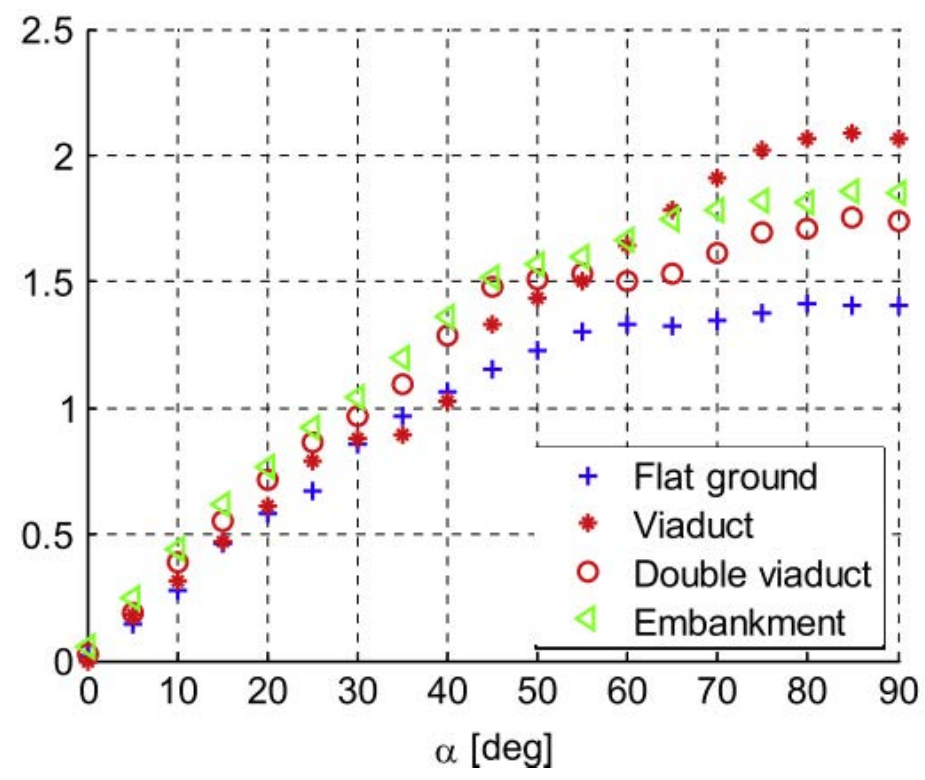

Abb. 5-13: Luftwiderstandsbeiwert in Querrichtung über Anströmwinkel für verschiedene Fahrsituationen [CHE11]

Die vorgestellten Quellen offenbaren hinsichtlich der Definition des Luftwiderstandsbeiwerts in Querrichtung eine breite Spanne möglicher Werte. Vor dem Hintergrund des Fehlens umfassender aerodynamischer Daten wird für das zur Untersuchung der Kippstabilität der Fahrzeugmodelle verwendete Seitenwindmodell der in [BLY08] empfohlene konservative Ansatz verfolgt, der die Wahl eines Wertes im oberen Bereich des Spektrums vorsieht. Zieht man zusätzlich in Betracht, dass Brücken einen erheblichen Anteil der Charakteristika bei LeichtLkw-Unfällen ausmachen (Kapitel 4.2.2.2), so ist insgesamt die Wahl eines Luftwiderstandsbeiwerts in Querrichtung von 1,8 für die Fahrzeugmodelle als sinnvoll anzusehen.

In [PRO15], einer Studie zur Seitenwindbelastung von Kraftfahrzeugen beim Befahren der Fehmarnsundbrücke, wird eine Schräganströmung zwischen $30^{\circ}$ und $60^{\circ}$ zur Längsrichtung im Vergleich zu einem $90^{\circ}$ angreifenden Wind als aerodynamisch kritischer bewertet. Bei dem hier verwendeten Windmodell werden Böen nachgebildet, die aus vor Ort gemessenen Windverläufen abgeleitet sind. Die Windgeschwindigkeiten werden mittels aerodynamischer Parameter aus der Literatur in Windbelastungen für verschiedene Fahrzeugtypen umgerechnet. Hinsichtlich Lkw ohne Anhänger werden die in Abb. 5-14 dargestellten Luftwider- 
standsbeiwerte zugrunde gelegt. Es ist ein Anstieg des Faktors in Querrichtung $\left(\mathrm{C}_{\mathrm{Fy}}\right)$ bis $\mathrm{zu}$ einem Windangriffswinkel von $50^{\circ}$ zur Längsrichtung zu erkennen. Danach pendelt sich der Luftwiderstandsbeiwert bei etwa 1,2 ein. Demnach wäre zumindest in Bezug auf Lkw ohne Anhänger eine Windanströmung unter $50^{\circ}$ bis $90^{\circ}$ als kritischster Bereich anzusehen, was somit nicht im Widerspruch zu den im Rahmen dieses Projekts getroffenen Annahmen steht. Im Zuge der Parametervariationen in Kapitel 5.5.4 erfolgen Simulationen, in denen auch eine Schräganströmung für das definierte Seitenwindmodell abgebildet wird. Dazu wird der Windkraftvektor in eine quer- und eine längsgerichtete Komponente zerlegt.

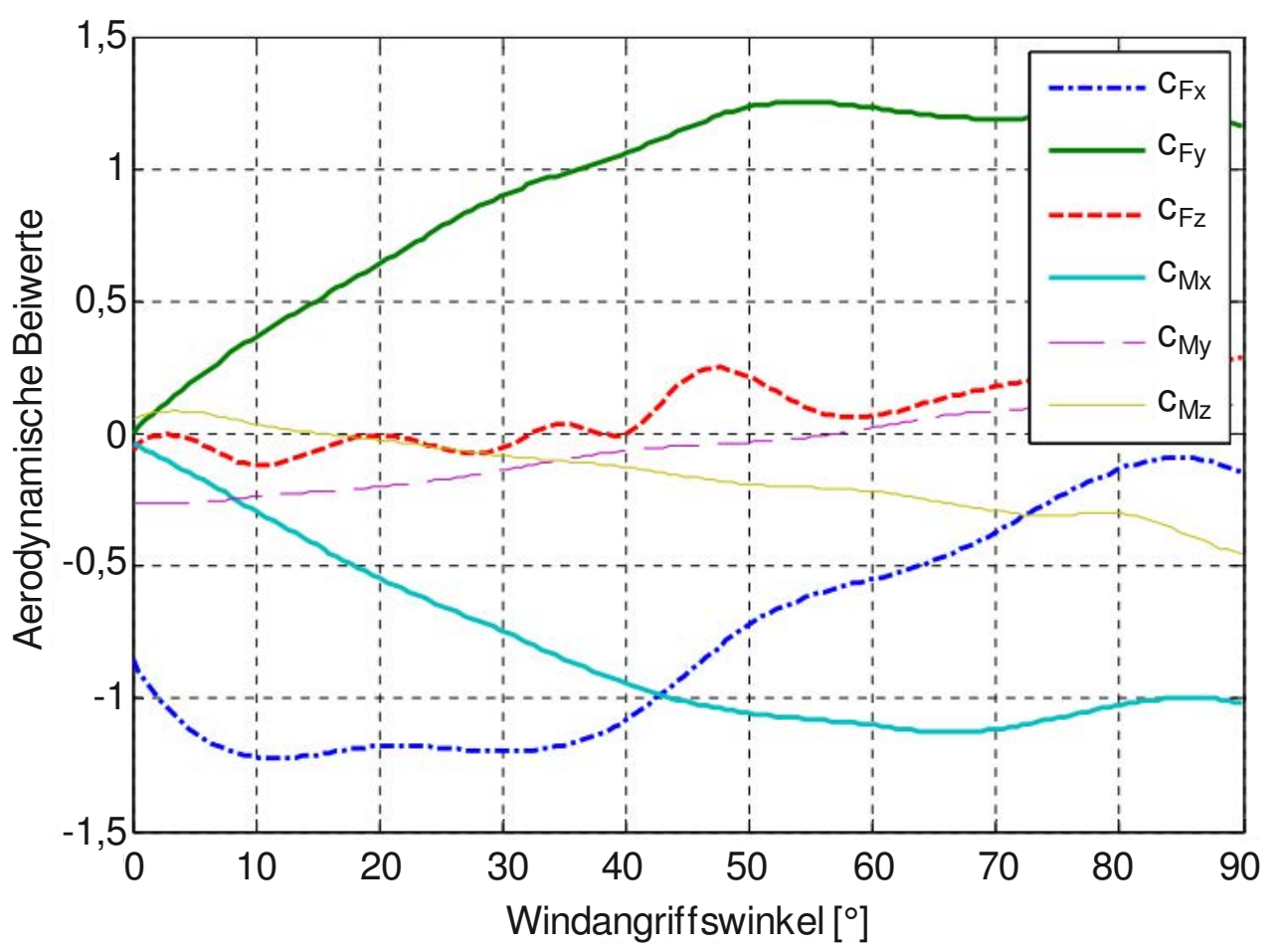

Abb. 5-14: Aerodynamische Beiwerte für Lkw ohne Anhänger nach [PRO15]

Die Windkraft greift immer im Flächenmittelpunkt der Aufbauten der Fahrzeugmodelle an. Dabei werden verschiedene Seitenwindbelastungen abgebildet, welche in Abb. 5-15 veranschaulicht sind. Es wird zwischen einer konstanten Windböe (direkter Wind), der Einfahrt des Gespanns in den Wind (aufbauender Wind) und einer sinusförmigen Windanregung (alternierender Wind) unterschieden. Die Windkraft wird jeweils nach drei Sekunden auf das eingeschwungene und in Geradeausfahrt befindliche Fahrzeug aufgegeben. Dies wird in der Simulation durch rote Pfeile im Flächenmittelpunkt von Zugfahrzeug und Anhänger visualisiert. Die damit verbundene Mechanik des Kippvorgangs geht aus Abb. 9-28 im Anhang hervor.

Der direkte Wind (Abb. 5-15 links) entspricht einer plötzlich über der gesamten Fahrzeuglänge auftretenden Windböe mit konstanter Stärke und Dauer. Dieses Windmodell wird auch im Rahmen der Parametervariationen in Kapitel 5.5.4 verwendet. Beim aufbauenden Wind (Abb. 5-15 mittig) fährt der Gliederzug zunächst im Windschatten einer seitlichen Bebauung, um dann nach drei Sekunden Fahrtzeit hinter der Bebauung in den mit konstanter Stärke und Dauer wehenden Wind einzufahren. 


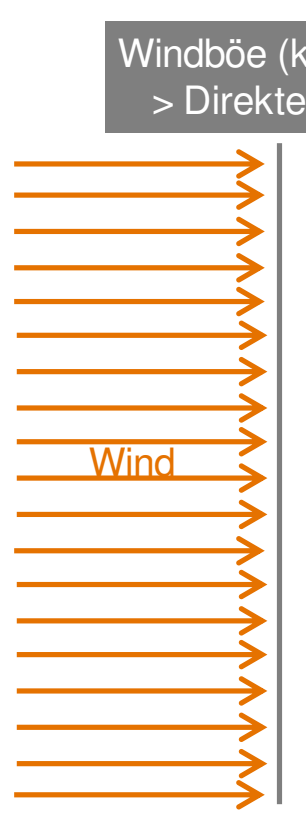

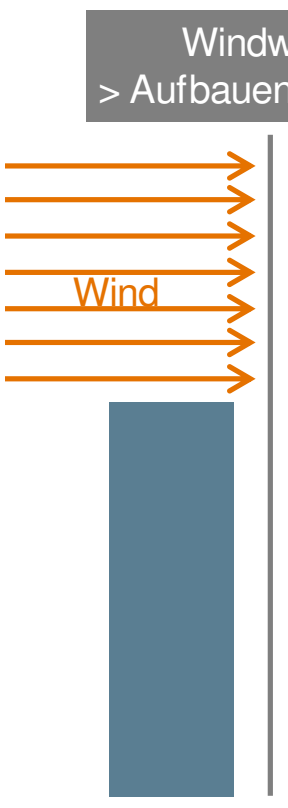

Windwand

Aufbauender Wind

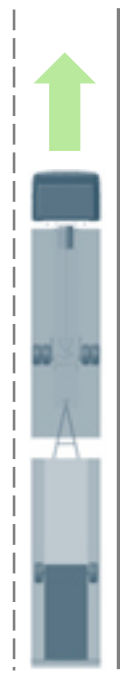

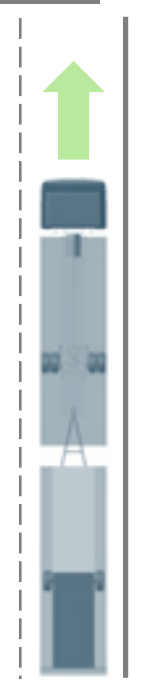

Windböe (Sinus)

$>$ Alternierender Wind

Abb. 5-15: Simulierte Seitenwindbelastungen

Dieses Einfahren in den Wind stellt auch mögliche kritische Situationen beim Befahren von Brücken nach. Hierbei wird zunächst nur das Zugfahrzeug mit Wind beaufschlagt, bevor durch die sich stetig vergrößernde windbeaufschlagte Fläche auch der Anhänger erfasst wird, d.h. bei Einfahrt in den Wind ist die auf das Gespann wirkende Windkraft $F_{\text {sw }}(t)$ abhängig von der Zeit (Abb. 5-16). Die im Wind befindliche Strecke $L_{x}(t)$ des Gliederzugs steigt linear mit der Fahrzeuggeschwindigkeit $\mathrm{v}_{\mathrm{Fzg}}$ und der Zeit $t$ an, sodass während dieser Phase auch die Windbelastung immer weiter steigt. Zusätzlich zu dieser Querkraft wird dabei ein Moment $\mathrm{M}_{\mathrm{sw}}(\mathrm{t})$ um die Hochachse von Zugfahrzeug und Anhänger hervorgerufen. Da der Kraftangriffspunkt der Windlast stets im Flächenschwerpunkt der Fahrzeugmodelle liegt, muss dieses Moment separat definiert werden. Es berechnet sich aus der aktuellen Windkraft $F_{s w}(t)$ und dem Hebelarm $S_{x}(t)$ zwischen dem eigentlichen Windangriffspunkt (Mittelpunkt blaue Fläche) und dem jeweiligen Flächenschwerpunkt des Aufbaus, wie aus den Gleichungen in Abb. 5-16 hervorgeht.

$$
\begin{aligned}
& \Delta p_{s w}=1 / 2 \rho v_{\text {Wind }^{2}} \\
& \mathrm{~L}_{x}(t)=v_{\text {Fzg }} \Delta t \\
& F_{s w}(t)=\Delta p_{s w} L_{x}(t) H c_{w} \\
& S_{x}(t)=\frac{L-L_{x}(t)}{2} \\
& M_{s w}(t)=F_{s w}(t) S_{x}(t)
\end{aligned}
$$
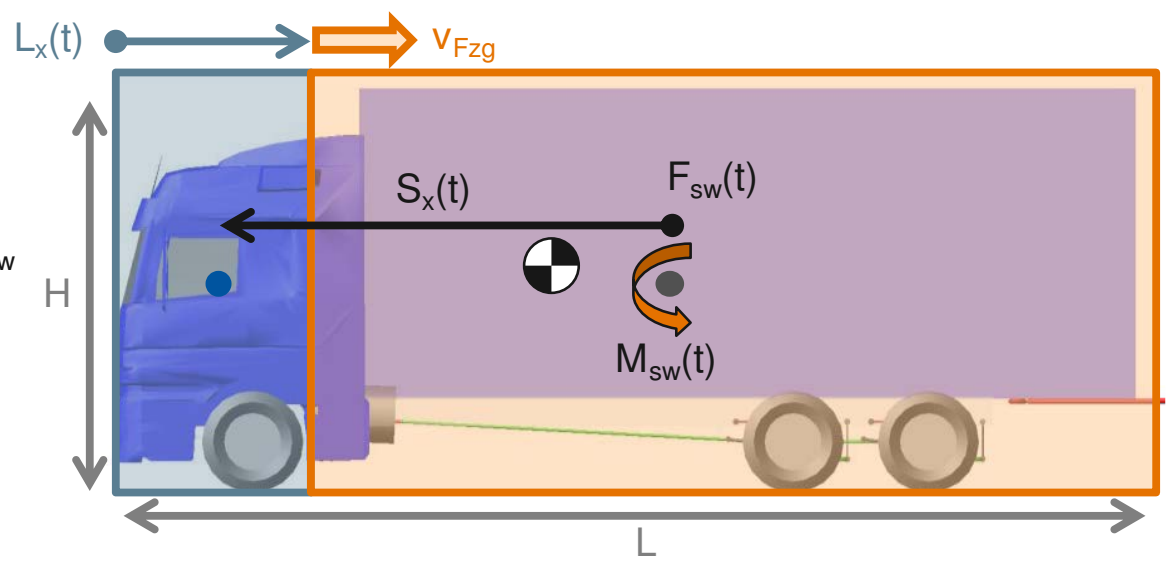

Abb. 5-16: Ansatz zur Abbildung des Einfahrens des Gliederzugs in den Seitenwind 
Bei alternierendem Wind (Abb. 5-15 rechts) wird der direkten Windkraft ein Sinussignal überlagert, welches sich zwischen null und der betrachteten Windgeschwindigkeit $\left(v_{\text {Wind }}\right)$ bewegt und dabei von $0,1 \mathrm{~Hz}$ auf $10 \mathrm{~Hz}$ gesteigert wird. So kann bewertet werden, ob es zu einem kritischen Aufschwingen des Systems bei den jeweiligen Windgeschwindigkeiten kommt.

Die in der Simulation abgebildeten Windgeschwindigkeiten reichen von 5 bis $40 \mathrm{~m} / \mathrm{s}$ (18 bis $144 \mathrm{~km} / \mathrm{h}$, vgl. Abb. 4-34) und repräsentieren jeweils die mittlere Windgeschwindigkeit der auf das Fahrzeug wirkenden Böe.

\subsection{Simulationsparameter}

Neben der Windgeschwindigkeit werden weitere Parameter variiert. So werden mit 30, 50, 70 und $85 \mathrm{~km} / \mathrm{h}$ verschiedene Fahrzeuglängsgeschwindigkeiten berücksichtigt und zusätzlich auch Simulationen mit einem stehenden Gespann $\left(v_{\mathrm{Fzg}}=0 \mathrm{~km} / \mathrm{h}\right)$ unter Seitenwindbelastung durchgeführt (Kapitel 5.5.4.1). Dabei stellt $85 \mathrm{~km} / \mathrm{h}$ das obere Spektrum für Güterkraftfahrzeuge dar und bildet somit die für die Simulationen maßgebliche Fahrzeuggeschwindigkeit.

Ein weiterer grundlegender Simulationsparameter ist der Beladungszustand. Im Rahmen der Untersuchungen zur Kippstabilität bei Seitenwind erfolgen für die jeweiligen Gliederzüge sowohl Simulationen im unbeladenen als auch im voll beladenen Zustand (Abb. 5-17).

\begin{tabular}{|c|c|c|c|}
\hline & Beladungszustand & Gewicht [t] & Schwerpunkthöhe [m] \\
\hline 40 t-Gliederzug & Unbeladen & 10,2 & 0,846 \\
\hline Zugfahrzeug & Voll beladen & 22 & 1,475 \\
\hline 40 t-Gliederzug & Unbeladen & 4,5 & 0,915 \\
\hline Anhänger & Voll beladen & 18 & 1,55 \\
\hline 12 t-Gliederzug & Unbeladen & 5 & 0,762 \\
\hline Zugfahrzeug & Voll beladen & 7,5 & 1,3 \\
\hline 12 t-Gliederzug & Unbeladen & 2,2 & 1,48 \\
\hline Anhänger & Voll beladen & 4,4 & 1,93 \\
\hline
\end{tabular}

Abb. 5-17: Abbildung verschiedener Beladungszustände

Im Zuge der durchgeführten Parametervariationen werden für den 12 t-Gliederzug auch ungleiche Beladungszustände betrachtet, d.h. nur Zugfahrzeug bzw. nur Anhänger beladen (Kapitel 5.5.4.3). Dabei werden die in Abb. 5-17 für die jeweiligen Beladungszustände aufgeführten Gewichte und Schwerpunkthöhen entsprechend kombiniert. Die Schwerpunkthöhe im beladenen Zustand berechnet sich aus den Schwerpunkthöhen des leeren Fahrzeugs bzw. Anhängers und einer homogen über die gesamte Ladeflächenlänge und Höhe verteilten Ladung. Des Weiteren wird für den voll beladenen $12 \mathrm{t}$-Gliederzug eine Auflastung des Anhängers um $2 \mathrm{t}$, wie in Kapitel 2.2 angeführt, berücksichtigt (Kapitel 5.5.4.5) und darüber hinaus auch der Einsatz von Ballasttanks bei Leerfahrten untersucht (Kapitel 5.5.4.10).

Einfluss auf die Fahrdynamik hat auch die Reaktion des Fahrers auf die plötzlich auftretende Seitenwindbelastung. Standardmäßig wird in den durchgeführten Simulationen das Fahrer- 
regler-Modell von Adams/Car verwendet. Der Fahrerregler soll dabei trotz Seitenwindeinfluss eine Geradeausfahrt einstellen. Die Auswertung der Simulationen hat gezeigt, dass die maximalen Lenkwinkelanforderungen hierbei nicht über einer Lenkwinkelrate von $90 \%$ s liegen. Bei Fahrdynamikversuchen werden Fahrern Lenkwinkelraten von 300 bis $500 \%$ s erlaubt. Demnach ist die seitens des Reglermodells erreichte Lenkwinkelrate im Hinblick auf einen realen Fahrer als gut umsetzbar einzustufen. Allerdings reagiert der Fahrerregler bereits nach einer Latenzzeit von 20 ms auf den auftretenden Seitenwindeinfluss. Hier ist in der Realität von einer längeren Reaktionszeit des Fahrers auszugehen. Zudem ist offen, wie früh der Fahrer die angreifende Seitenwindkraft, gerade am Anhänger, wahrnimmt. Um den Einfluss des Fahrerreglers auf die Fahrdynamik bzw. die Kippgrenze bewerten zu können, werden in Kapitel 5.5.4.2 daher zusätzlich auch Simulationen mit einem ungeregelten Modell durchgeführt. Dabei erfolgt keine Lenkbewegung seitens des Fahrers, vielmehr wird ein konstanter Lenkwinkel von $0^{\circ}$ gehalten.

Zu den im Vorfeld untersuchten fahrzeugmodellseitigen Parametern zählen die Federsteifigkeiten, die Deichselsteifigkeit und Reibung sowie der Reibwert zwischen Reifen und Straße. Die verwendeten Simulationsmodelle verfügen an der Vorderachse des Zugfahrzeugs über eine Blattfederung und für die Aufbauten von Zugfahrzeug und Anhänger jeweils über getrennte Luftfedersysteme, die das Niveau der Aufbauten achsindividuell und in Abhängigkeit des Beladungszustands auf die geforderte Höhe einstellen. Eine Variation der Federsteifigkeiten im Zuge einer Sensitivitätsanalyse zeigt keinen relevanten Einfluss auf das Kippverhalten der Fahrzeugmodelle.

Der in die Fahrzeugmodelle implementierte Anschlag der Deichselanbindung zwischen Zugfahrzeug und Anhänger wird über eine aus Konstruktionsdaten abgeleitete Steifigkeit abgebildet, die anhand einer weiteren Sensitivitätsanalyse validiert worden ist. Dabei zeigt sich ebenfalls kein relevanter Einfluss auf das Kippverhalten des Anhängers.

Das Reifenverhalten wird mittels des Pacejka Magic-Formula Reifenmodells abgebildet. Dieses Modell basiert auf einem empirischen Ansatz, der die Reifenparameter über definierte Formeln beschreibt. Es eignet sich besonders gut, um fahrdynamische Manöver auf ebener Strecke abzubilden [ADA14]. Für den vorliegenden Anwendungsfall ist das Pacejka Reifenmodell den komfortorientierten F-Tyre Modellen sowohl hinsichtlich der Güte der berechneten Reifenkräfte als auch in Bezug auf die benötigten Rechenzeiten vorzuziehen. Der Reibwert zwischen Straße und Reifen ist als Standard bei allen Fahrmanövern auf 1 gesetzt. Auch hier ergibt eine Sensitivitätsuntersuchung, dass für Werte zwischen 0,9 und 1,0 keine Unterschiede im Kippverhalten der Fahrzeugmodelle festzustellen sind.

Weitere Parametervariationen werden in Hinblick auf das Seitenwindmodell vorgenommen. Neben einer genaueren Betrachtung der Fahrdynamik des leeren 12 t-Gliederzugs bei überkritischen Windgeschwindigkeiten (108, 126 und 144 km/h, Kapitel 5.5.4.7), erfolgen auch Simulationen unter Schräganströmung (Kapitel 5.5.4.8) sowie Simulationen, bei denen der direkte Wind nur am Zugfahrzeug des leeren Gespanns angreift, wodurch ein Anhänger mit geöffneter Plane abgebildet wird (Kapitel 5.5.4.9). 
Zur Untersuchung des Einflusses von Sturmstützen (siehe Kapitel 2.2) sowie einer zweiten zentralen Anhängerachse auf die Kippstabilität werden zusätzlich zu den reinen Parametervariationen auch entsprechende Anpassungen am Fahrzeugmodell des $12 \mathrm{t}$-Gliederzuges vorgenommen, welche in Abb. 5-18 und Abb. 5-19 dargestellt sind.

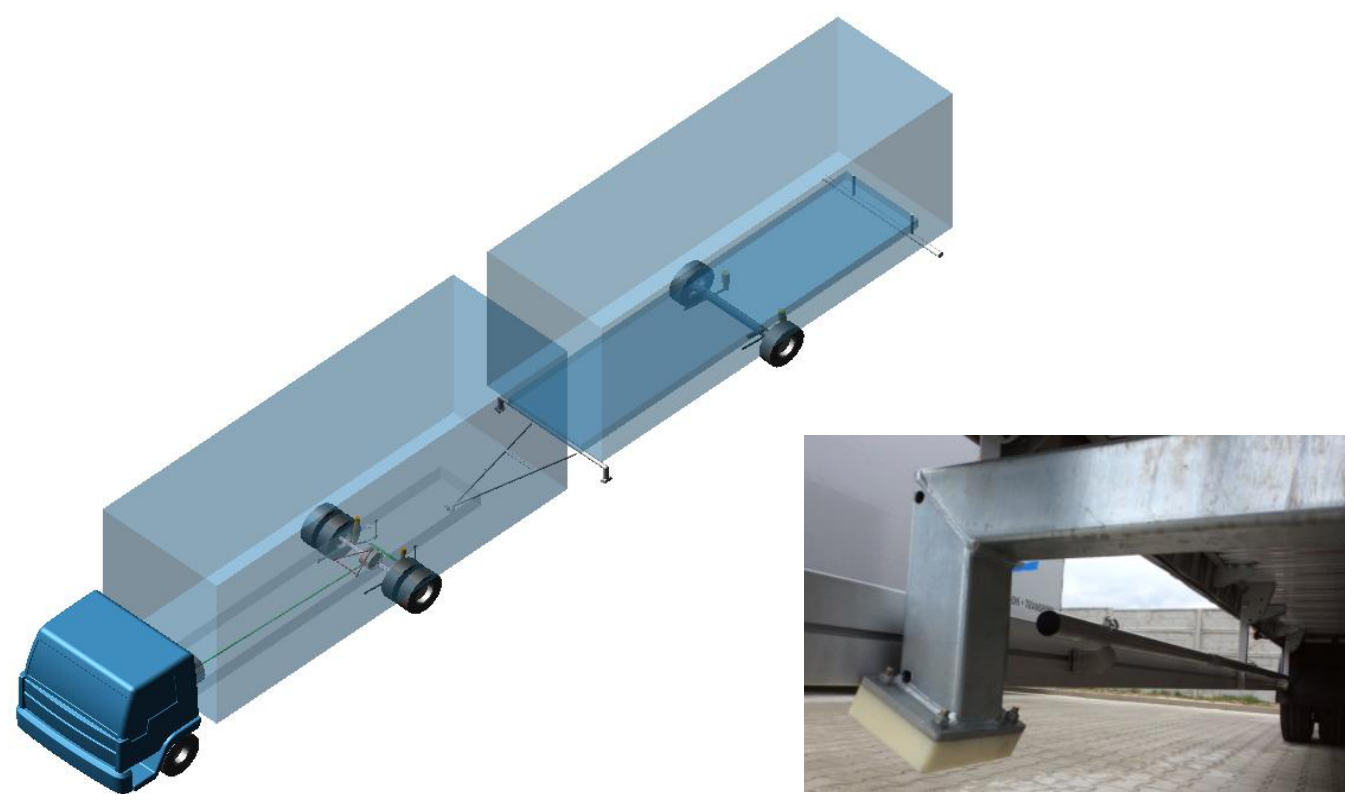

Abb. 5-18: Implementierung von Sturmstützen in das Modell des 12 t-Gliederzuges

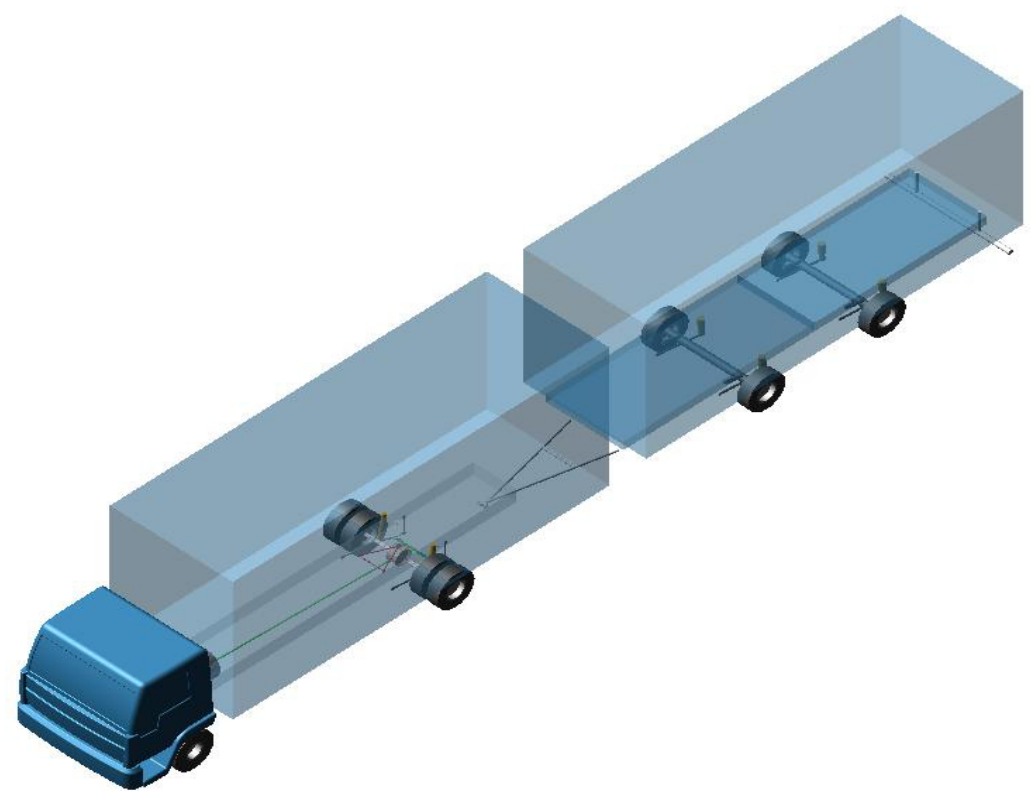

Abb. 5-19: Implementierung einer zweiten zentralen Anhängerachse in das Modell des 12 tGliederzuges

Die Implementierung der Sturmstützen beruht auf realen Konstruktionsdaten. Es handelt sich hierbei um eine starre Querverstrebung vorne entlang der kompletten Anhängerbreite mit 
beidseitigen Auflageflächen, die im Falle eines Kippens des Anhängers jeweils als erstes Element Kontakt zum Boden aufbauen. Durch eine entsprechende Kontaktdefinition entsteht ein starrer Kontakt zwischen Straße und Auflagefläche der jeweiligen Stütze. Die Simulationsergebnisse des Fahrzeugmodells mit implementierten Sturmstützen werden in Kapitel 5.5.3 beschrieben. Inwieweit eine zweite zentrale Anhängerachse die Kippgefahr des 12 t-Gliederzuges reduzieren kann, wird in Kapitel 5.5.4.4 untersucht.

Insgesamt ergeben sich im Zuge der berücksichtigten Bandbreite der Windgeschwindigkeiten sowie der zahlreichen Parametervariationen über 500 Simulationen, die innerhalb dieses Projekts durchgeführt worden sind.

\section{$5.4 \quad$ Simulationsauswertung}

Das Hauptkriterium bei der Auswertung der Simulationen ist die Beurteilung der Kippstabilität der Fahrzeugmodelle. Dabei wird für die jeweiligen Randbedingungen ermittelt, bei welcher Windgeschwindigkeit es zu einem Kippen des Anhängers bzw. des gesamten Gespanns kommt. Neben dem reinen Kippen werden auch verschiedene fahrdynamische Parameter im Rahmen der Auswertung betrachtet. Dazu zählen die Spurabweichung, die Radlastverschiebung sowie das Wankverhalten des Fahrzeugs.

Es kommt zu einem Spurversatz des Fahrzeugs, wenn die von den Reifen aufgebrachte Seitenkraft nicht mehr ausreicht, um der durch den Wind aufgebrachten Querkraft entgegenzuwirken. Das Querkraftpotential der Reifen ist abhängig vom Gesamtbelastungszustand der Reifen, d.h. entscheidend ist die gleichzeitig übertragene Längskraft. Je höher die Längskraft, die mit der Fahrzeuglängsgeschwindigkeit zunimmt, desto geringer ist das am Reifen noch zur Verfügung stehende Querkraftpotential. Der seitliche Spurversatz steigt also nicht nur mit der Windkraft, sondern auch mit der Fahrzeuggeschwindigkeit. Die Spurabweichung des Fahrzeug- bzw. Anhängerschwerpunktes in Querrichtung wird ab einem Wert von 0,5 m als kritisch eingestuft. Auf Autobahnen in Deutschland beträgt die Fahrspurbreite 3,75 m. Bei einer Fahrzeugbreite von 2,55 m und unter Annahme, dass sich das Fahrzeug mittig in der Spur befindet, bleiben noch 0,6 m Sicherheitsreserve in beide Richtungen. Schwenkt der Anhänger oder das Fahrzeugheck zusätzlich noch aus, ist diese Sicherheitsreserve schnell aufgebraucht. In [PRO15] wird ein Spurversatz ab 0,5 m ebenfalls als kritisch bewertet.

Durch die angreifende Windkraft kommt es zu einer Radlastverlagerung, die im ungünstigsten Fall zur vollständigen Entlastung der windzugewandten Räder führt. Bei Radlastverlust entfallen einseitig alle Reifenkräfte. Dadurch wird das Fahrzeug instabil und für den Fahrer nur noch schwer kontrollierbar. Zusätzlich kommt es aufgrund der wegfallenden Querkräfte an den abgehobenen Rädern zu einer Erhöhung des Spurversatzes. Deshalb wird für die entsprechenden Simulationen stets die Zeit bis zum Radlastverlust mit ausgewertet.

Der Wankwinkel beschreibt die Drehbewegung des Fahrzeugs um dessen Längsachse. Sowohl die Schwerpunktverschiebung der Fahrzeugaufbauten in Richtung der windabgewandten Fahrzeugseite während der Phase bis zum Radlastverlust als auch ein mögliches nachfolgendes Kippen lassen sich mittels des Wankwinkels charakterisieren. 


\subsection{Ergebnisse}

Im Folgenden werden die Simulationsergebnisse in kompakter Form vorgestellt. Das Hauptkriterium bildet dabei die Frage, ob das Gespann unter den jeweiligen Randbedingungen kippt und ab welcher Windgeschwindigkeit dies geschieht. Angegeben werden zudem der maximale Spurversatz sowie die Zeit vom Einsetzen der Windlast bis zum Radlastverlust. Wie in Kapitel 5.4 bereits diskutiert, wird ein Spurversatz unterhalb von einem halben Meter noch nicht als kritisch bewertet (grüne Einstufung). Demgegenüber werden Simulationen mit einer Spurabweichung von mindestens $0,5 \mathrm{~m}$ als kritisch bzw. gelb eingestuft. Sofern es hierbei auch zu einem Kippen von Anhänger bzw. Zugmaschine kommt, erfolgt eine rote Einstufung. Mittels dieser Farbcodierung lässt sich das Verhalten des jeweiligen Gespanns und Beladungszustands über dem berücksichtigten Windgeschwindigkeitsbereich übersichtlich darstellen. Eine entsprechende Zusammenfassung gibt Abb. 9-29 im Anhang.

\subsubsection{Direkter Wind}

Beim direkten Wind wird das auf einer geraden Straße ohne seitlichen Bewuchs oder sonstige Windbarrieren fahrende Gespann nach drei Sekunden mit einer konstanten und sowohl an Zugfahrzeug als auch Anhänger angreifenden seitlichen Windlast beaufschlagt (Kapitel 5.2). Das aus dieser Windlast resultierende fahrdynamische Verhalten wird zunächst für die beiden berücksichtigten 40 t-Gliederzüge betrachtet.

\subsubsection{1 $\quad 40 \mathrm{t}$-Gliederzug}

Die beiden Fahrzeugmodelle des 40 t-Gliederzuges, d.h. die Variante mit Gelenkdeichselanhänger sowie die Variante mit Starrdeichselanhänger (Kapitel 5.1), verhalten sich im Allgemeinen sehr ähnlich. Auch wenn die Ergebnisse für den Starrdeichselanhänger minimal kritischer ausfallen, ergeben sich hinsichtlich der Farbbewertung keine Unterschiede. Eine Übersicht für den beladenen und unbeladenen Zustand geht aus Abb. 5-20 hervor.

\begin{tabular}{|c|c|c|c|c|}
\hline \multirow{2}{*}{$\begin{array}{l}\text { Wind- } \\
\text { stärke }\end{array}$} & \multicolumn{2}{|c|}{ VWind } & \multicolumn{2}{|c|}{ Direkter Wind } \\
\hline & {$[\mathrm{m} / \mathrm{s}]$} & {$[\mathrm{km} / \mathrm{h}]$} & Leer & Beladen \\
\hline 7 & 15 & 54 & $s=0,38 / 0,38 \mathrm{~m}$ & \\
\hline 7 & 16 & 57,6 & & \\
\hline 8 & 20 & 72 & $s=0,73 / 0,7 \mathrm{~m}$ & $s=0,12 / 0,1 \mathrm{~m}$ \\
\hline 9 & 21 & 75,6 & & \\
\hline 9 & 22 & 79,2 & & \\
\hline 9 & 23 & 82,8 & $s=1,0 / 0,97 \mathrm{~m}$ & \\
\hline 9 & 23,5 & 84,6 & $s=1,1 / 1,0 \mathrm{~m}$ & \\
\hline 9 & 24 & 86,4 & $t=0,09 / 0,1 \mathrm{~s}$ & \\
\hline 10 & 25 & 90 & $t=0,09 / 0,1 \mathrm{~s}$ & $s=0,36 / 0,36 \mathrm{~m}$ \\
\hline 11 & 30 & 108 & & $s=0,55 / 0,53 \mathrm{~m}$ \\
\hline 12 & 35 & 126 & & $s=0,79 / 0,69 \mathrm{~m}$ \\
\hline 12 & 40 & 144 & & $s=1,2 / 1,1 \mathrm{~m}$ \\
\hline
\end{tabular}

Unkritisch

Kritisch

Kippen

$s=$ Spurversatz

$t=$ Zeit bis zum

Radlastverlust

Starr-/Gelenkdeichselanhänger

Abb. 5-20: Ergebnisse des 40 t-Gliederzugs für eine Fahrzeuggeschwindigkeit von 85 km/h 
Die Werte der zugehörigen fahrdynamischen Parameter sind sowohl für die Starrdeichselanhänger- als auch für die Gelenkdeichselanhängervariante angegeben. Während der $40 \mathrm{t}$ Gliederzug im unbeladenen Zustand ab einer Windgeschwindigkeit von $24 \mathrm{~m} / \mathrm{s}(86 \mathrm{~km} / \mathrm{h})$ kippt (Abb. 5-21 \& Abb. 5-22), kommt es im beladenen Zustand bis zur definierten maximalen Windgeschwindigkeit von $40 \mathrm{~m} / \mathrm{s}(144 \mathrm{~km} / \mathrm{h})$ zu keinem Kippen des Gespanns. Allerdings liegen hier für beide Anhängervarianten Spurabweichungen von mehr als einem Meter vor, wobei es bereits ab Windgeschwindigkeiten von $30 \mathrm{~m} / \mathrm{s}(108 \mathrm{~km} / \mathrm{h}) \mathrm{zu}$ kritischen Spurabweichungen kommt. Im unbeladenen Zustand ist dies für Windgeschwindigkeiten oberhalb von $16 \mathrm{~m} / \mathrm{s}(58 \mathrm{~km} / \mathrm{h})$ der Fall.

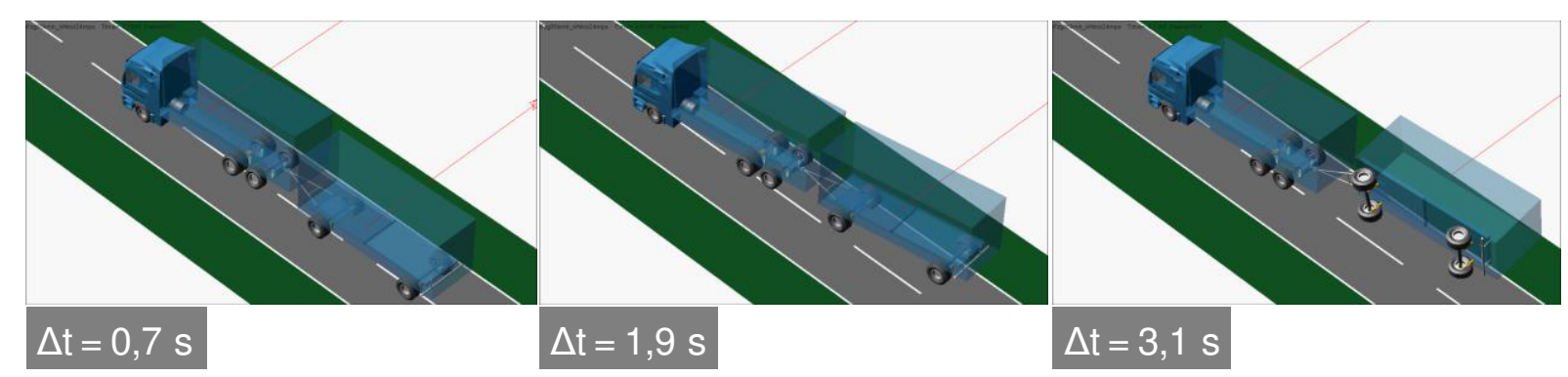

Abb. 5-21: Unbel. 40 t-Zug, Gelenkdeichsel $\left(\mathrm{v}_{\mathrm{Fzg}}=85 \mathrm{~km} / \mathrm{h}, \mathrm{v}_{\text {Wind_direkt }}=24 \mathrm{~m} / \mathrm{s}(86 \mathrm{~km} / \mathrm{h})\right)$

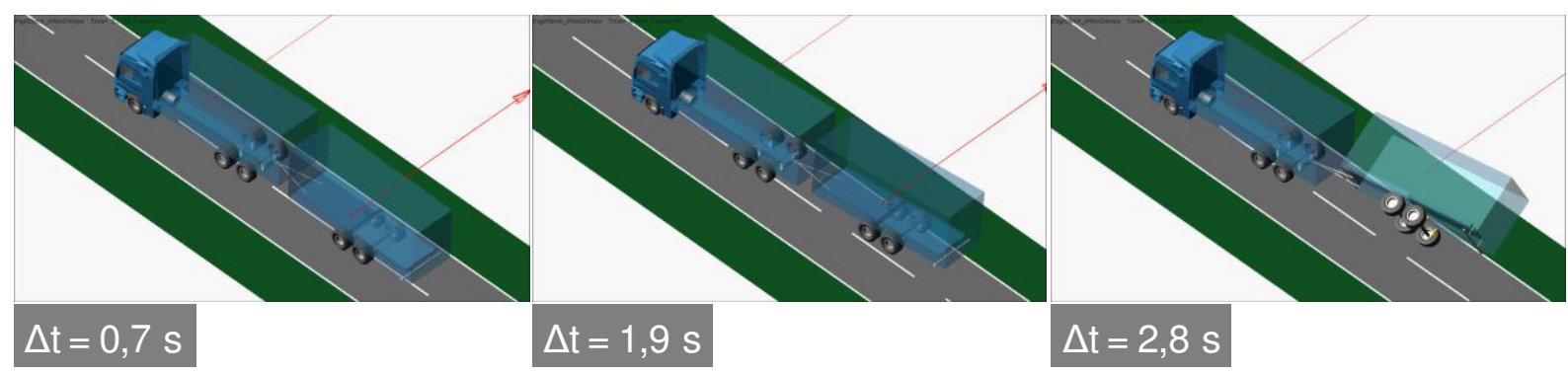

Abb. 5-22: Unbeladener $40 \mathrm{t}-Z u g$, Starrdeichsel $\left(\mathrm{v}_{\mathrm{Fzg}}=85 \mathrm{~km} / \mathrm{h}, \mathrm{v}_{\text {Wind_direkt }}=24 \mathrm{~m} / \mathrm{s}(86 \mathrm{~km} / \mathrm{h})\right)$

Auch wenn es im unbeladenen Zustand bei einer Windgeschwindigkeit von $24 \mathrm{~m} / \mathrm{s}(86 \mathrm{~km} / \mathrm{h})$ unmittelbar nach Einsetzen des Windes, d.h. nach ca. 0,1 Sekunden (Abb. 5-20), zum Radlastverlust an der windzugewandten Seite des Anhängers kommt, vergehen bis zum vollständigen Kippen ca. drei Sekunden (Abb. 5-21 \& Abb. 5-22).

\subsubsection{2 $12 \mathrm{t}$-Gliederzug}

Beim $12 \mathrm{t}$-Gliederzug kommt es für das betrachtete Windgeschwindigkeitsspektrum auch im beladenen Zustand zu einem Kippen des Anhängers. Wie in Abb. 5-23 dargestellt, geschieht dies ab einer Windgeschwindigkeit von $21,5 \mathrm{~m} / \mathrm{s}(77 \mathrm{~km} / \mathrm{h})$. Zwei Windstärken eher, d.h. ab einer Windgeschwindigkeit von $16 \mathrm{~m} / \mathrm{s}$ (58 km/h), kippt der Anhänger der unbeladenen Kombination. Zudem ist die Zeitspanne bis zum Radlastverlust für den unbeladenen Anhänger mit $0,12 \mathrm{~s}$ wesentlich kürzer als für den beladenen Anhänger, wo diese 1,84 s beträgt. Für beide Beladungszustände kommt es bereits bei Windgeschwindigkeiten unterhalb der Kippgrenze zu hohen Spurabweichungen, sodass eine Windgeschwindigkeit von $16 \mathrm{~m} / \mathrm{s}$ für den 
beladenen Zustand ebenfalls als kritisch anzusehen ist. Bezogen auf den unbeladenen Zustand beginnt der fahrdynamisch kritische Bereich entsprechend früher.

\begin{tabular}{|c|c|c|c|c|}
\hline \multirow{2}{*}{$\begin{array}{l}\text { Wind- } \\
\text { stärke }\end{array}$} & \multicolumn{2}{|c|}{ VWind } & \multicolumn{2}{|c|}{ Direkter Wind } \\
\hline & {$[\mathrm{m} / \mathrm{s}]$} & {$[\mathrm{km} / \mathrm{h}]$} & Leer & Beladen \\
\hline 5 & 10 & 36 & $s=0,37 m$ & $\mathrm{~s}=0,1 \mathrm{~m}$ \\
\hline 7 & 15 & 54 & $s=0,98 \mathrm{~m}$ & $s=0,45 \mathrm{~m}$ \\
\hline 7 & 15,5 & 55,8 & $\mathrm{~s}=0,99 \mathrm{~m}$ & \\
\hline 7 & 16 & 57,6 & $t=0,12 \mathrm{~s}$ & $s=0,51 \mathrm{~m}$ \\
\hline 8 & 20 & 72 & $t=0,12 \mathrm{~s}$ & $s=0,84 \mathrm{~m}$ \\
\hline 9 & 21 & 75,6 & & $s=0,94 \mathrm{~m}$ \\
\hline 9 & 21,5 & 77,4 & & $t=1,84 \mathrm{~s}$ \\
\hline 9 & 22 & 79,2 & & $t=1,82 \mathrm{~s}$ \\
\hline
\end{tabular}

Unkritisch
Kritisch
Kippen
$s=$ Spurversatz
$t=$ Zeit bis zum
Radlastverlust

Abb. 5-23: Ergebnisse des $12 \mathrm{t}$-Gliederzugs für eine Fahrzeuggeschwindigkeit von $85 \mathrm{~km} / \mathrm{h}$

In Abb. 5-24 und Abb. 5-25 wird die Fahrdynamik im unbeladenen und beladenen Zustand jeweils für die Windgeschwindigkeit veranschaulicht, ab der es zum Kippen des Anhängers kommt.

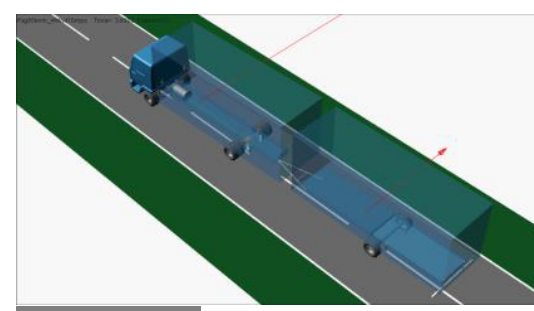

$\Delta t=0,7 \mathrm{~s}$

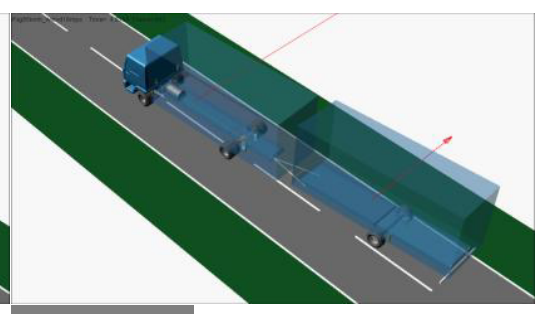

$\Delta t=1,9 \mathrm{~s}$

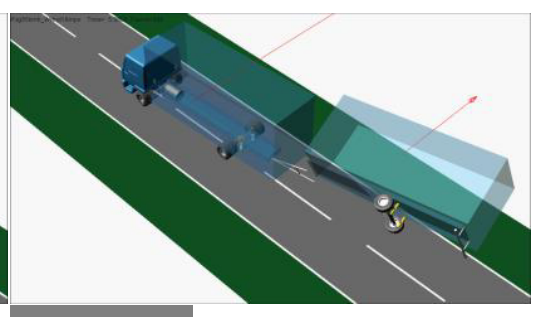

$\Delta t=3,0 s$

Abb. 5-24: Unbeladener $12 \mathrm{t}-\mathrm{Zug}\left(\mathrm{v}_{\mathrm{Fzg}}=85 \mathrm{~km} / \mathrm{h}\right.$, $\left.\mathrm{v}_{\text {Wind_direkt }}=16 \mathrm{~m} / \mathrm{s}(58 \mathrm{~km} / \mathrm{h})\right)$

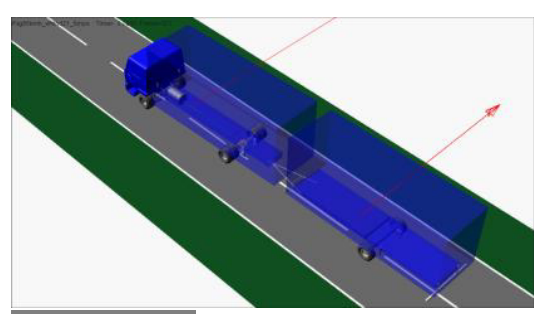

$\Delta t=0,7 \mathrm{~s}$

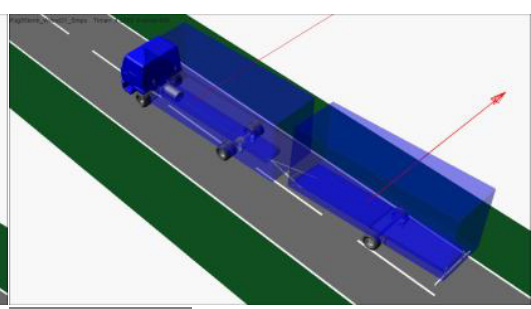

$\Delta \mathrm{t}=1,9 \mathrm{~s}$

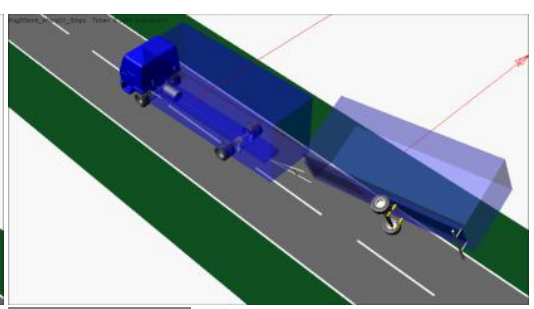

$\Delta t=3,2 s$

Abb. 5-25: Beladener $12 \mathrm{t}-Z u g\left(\mathrm{v}_{\mathrm{Fzg}}=85 \mathrm{~km} / \mathrm{h}\right.$, $\left.\mathrm{v}_{\text {Wind_direkt }}=21,5 \mathrm{~m} / \mathrm{s}(77 \mathrm{~km} / \mathrm{h})\right)$

\subsubsection{Aufbauender Wind}

In den Simulationen mit aufbauendem Wind fährt der Lastzug aus dem durch ein seitliches Hindernis verursachten Windschatten in einen konstanten Seitenwind hinein, wodurch sich die Windlast entsprechend der Fahrgeschwindigkeit von vorne nach hinten aufbaut bzw. 
erhöht und dabei gleichzeitig ein Moment um die Hochachse hervorruft (Kapitel 5.2). Auch für dieses Seitenwindmodell wird im Folgenden die Fahrdynamik der beiden $40 \mathrm{t}$-Gliederzüge sowie der 12 t-Leicht-Lkw-Kombination im beladenen und unbeladenen Zustand ausgewertet.

\subsubsection{1 $\quad 40 \mathrm{t}$-Gliederzug}

Die beiden Fahrzeugmodelle des 40 t-Gliederzuges, d.h. die Variante mit Gelenkdeichselanhänger sowie die Variante mit Starrdeichselanhänger (Kapitel 5.1), zeigen bezüglich des aufbauenden Windes ebenfalls kaum Unterschiede. Eine entsprechende Übersicht geht aus Abb. 5-26 hervor.

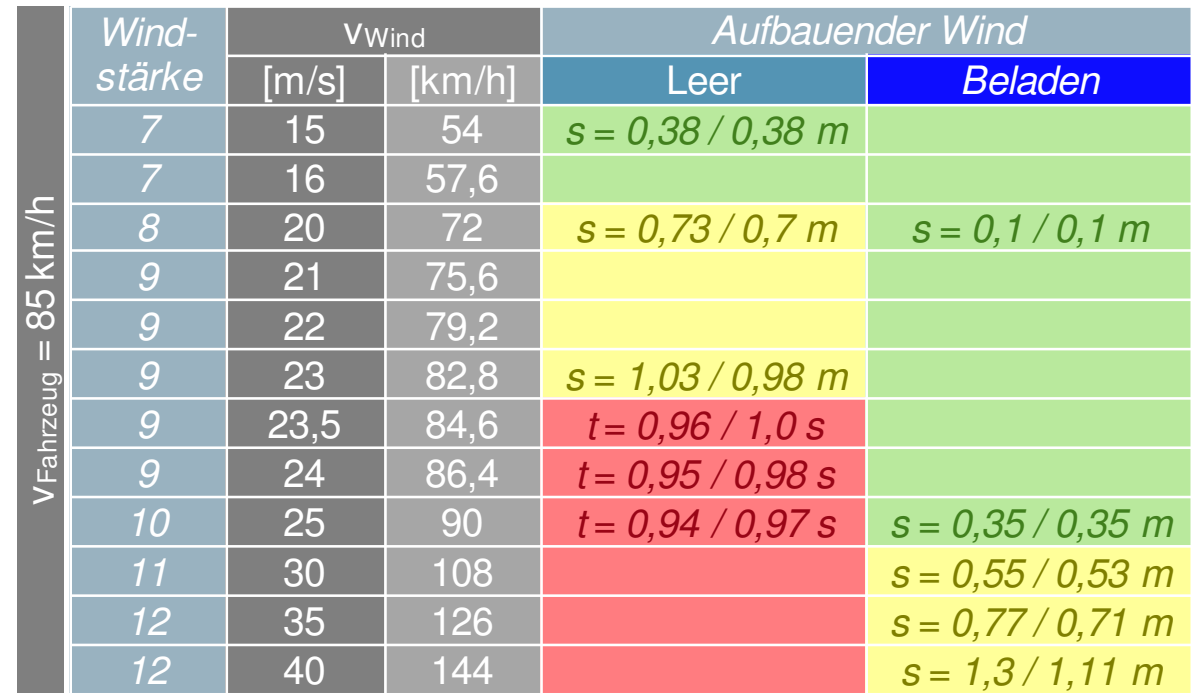

Unkritisch
Kritisch
Kippen
$s=$ Spurversatz
$t=$ Zeit bis zum
Radlastverlust
Starr- / Gelenk-
deichselanhänger

Abb. 5-26: Ergebnisse des 40 t-Gliederzugs für eine Fahrzeuggeschwindigkeit von 85 km/h

Im Vergleich zu den Simulationen mit direktem Wind ergibt sich ein weitestgehend analoges Verhalten. Wiederum kommt es im betrachteten Windgeschwindigkeitsbereich bis $40 \mathrm{~m} / \mathrm{s}$ für den $40 \mathrm{t}$-Gliederzug nur im unbeladenen Zustand zu einem Kippen des Anhängers. Dies erfolgt ab einer Windgeschwindigkeit von $23,5 \mathrm{~m} / \mathrm{s}(85 \mathrm{~km} / \mathrm{h})$ und damit etwas eher als im Falle des direkten Windes. Auch hinsichtlich des maximalen Spurversatzes stellen sich fast identische Werte ein. Lediglich die Zeit bis zum Radlastverlust fällt im Zuge des Hineinfahrens des Fahrzeugs in den Wind mit ca. $1 \mathrm{~s}$ deutlich länger aus.

In Abb. 5-27 und Abb. 5-28 wird das fahrdynamische Verhalten des unbeladenen 40 t-Gliederzuges für beide Anhängertypen und einer Windgeschwindigkeit von $24 \mathrm{~m} / \mathrm{s}$ veranschaulicht. Diese liegt unmittelbar über der Kippgrenze und ermöglicht einen direkten Vergleich mit den entsprechenden Simulationen zum direkten Wind in Abb. 5-21 und Abb. 5-22. Die angegebenen Zeitspannen beziehen sich auf den Zeitpunkt, ab dem das Zugfahrzeug aus dem Windschatten fährt, die Front sich also auf Höhe des Hindernisses befindet. Dieses ist jeweils im ersten Bild der dargestellten Bildfolgen unten rechts zu erkennen. Zum entsprechenden Zeitpunkt befindet sich der Anhänger größtenteils noch im windgeschützten Bereich. 


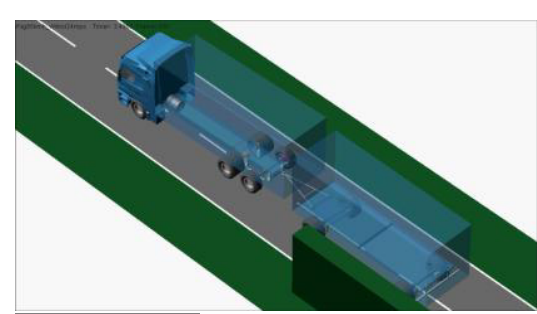

$\Delta t=0,5 \mathrm{~s}$

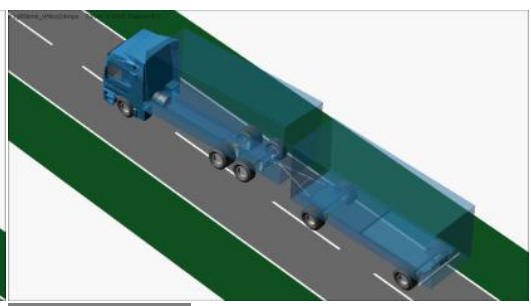

$\Delta t=1,5 \mathrm{~s}$

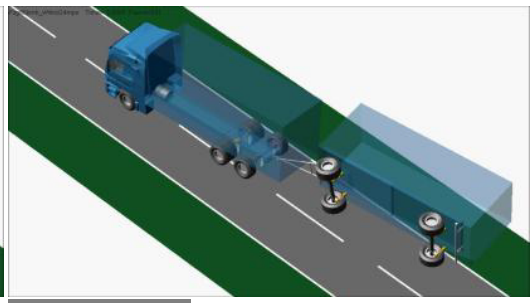

$\Delta t=2,8 \mathrm{~s}$

Abb. 5-27: Unbel. $40 \mathrm{t}-Z u g$, Gelenkdeichsel $\left(\mathrm{v}_{\mathrm{Fzg}}=85 \mathrm{~km} / \mathrm{h}\right.$, $\left.\mathrm{v}_{\text {Wind_autbauend }}=24 \mathrm{~m} / \mathrm{s}(86 \mathrm{~km} / \mathrm{h})\right)$

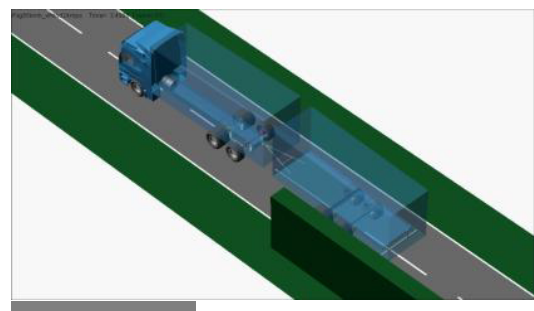

$\Delta \mathrm{t}=0,5 \mathrm{~s}$

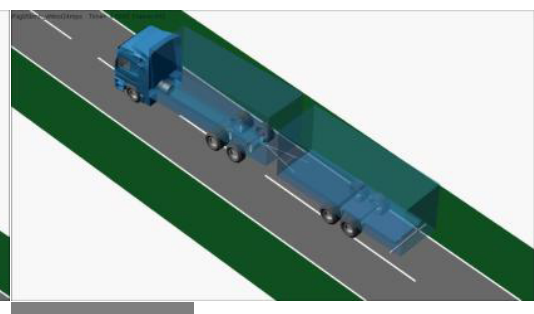

$\Delta t=1,5 \mathrm{~s}$

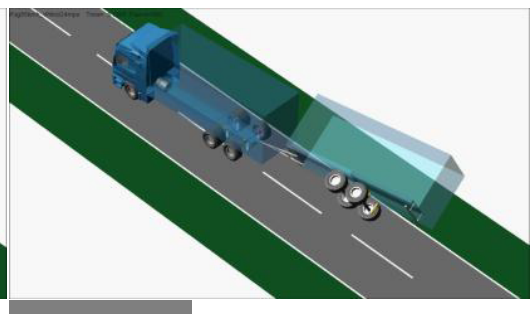

$\Delta t=2,8 \mathrm{~s}$

Abb. 5-28: Unbel. $40 \mathrm{t}-Z u g$, Starrdeichsel $\left(\mathrm{v}_{\mathrm{Fzg}}=85 \mathrm{~km} / \mathrm{h}, \mathrm{V}_{\text {Wind_aufbauend }}=24 \mathrm{~m} / \mathrm{s}(86 \mathrm{~km} / \mathrm{h})\right)$

Nach der Einfahrt in den Wind dauert es weniger als drei Sekunden bis zum Kippen des Anhängers, wobei es zuvor bereits zu hohen Spurabweichungen kommt.

\subsubsection{12 t-Gliederzug}

Das Seitenwindmodell des aufbauenden Windes sorgt auch bei der 12 t-Leicht-Lkw-Kombination für ein Kippen bei leicht geringerer Windstärke, d.h. ab einer Windgeschwindigkeit von $15,5 \mathrm{~m} / \mathrm{s}(56 \mathrm{~km} / \mathrm{h})$ im unbeladenen bzw. $20,5 \mathrm{~m} / \mathrm{s}(74 \mathrm{~km} / \mathrm{h})$ im beladenen Zustand und somit bei um 0,5 bzw. $1 \mathrm{~m} / \mathrm{s}$ niedrigeren Werten im Vergleich zu den Simulationen mit direktem Wind (Kapitel 5.5.1.2). In Windstärken gemessen kippt der beladene Leicht-Lkw bei aufbauendem Wind also bereits ab Windstärke 8, statt der zuvor beim direkten Wind ermittelten Windstärke 9. Eine Übersicht über die in den fahrdynamischen Übergangsbereichen auftretenden Spurabweichungen bzw. Zeiten bis zum Radlastverlust geht aus Abb. 5-29 hervor.

\begin{tabular}{|c|c|c|c|c|}
\hline \multirow{2}{*}{$\begin{array}{l}\text { Wind- } \\
\text { stärke }\end{array}$} & \multicolumn{2}{|c|}{ VWind } & \multicolumn{2}{|c|}{ Aufbauender Wind } \\
\hline & {$[\mathrm{m} / \mathrm{s}]$} & {$[\mathrm{km} / \mathrm{h}$} & Leer & Beladen \\
\hline 5 & 10 & 36 & $s=0,37 \mathrm{~m}$ & $s=0,1 \mathrm{~m}$ \\
\hline 7 & 15 & 54 & $s=0,91 \mathrm{~m}$ & $s=0,44 \mathrm{~m}$ \\
\hline 7 & 15,5 & 55,8 & $t=1,47 \mathrm{~s}$ & \\
\hline 7 & 16 & 57,6 & $t=1,22 \mathrm{~s}$ & $s=0,5 \mathrm{~m}$ \\
\hline 8 & 20 & 72 & $t=1,2 \mathrm{~s}$ & $s=0,84 \mathrm{~m}$ \\
\hline 8 & 20,5 & 73,8 & & $t=1,58 \mathrm{~s}$ \\
\hline 9 & 21 & 75,6 & & $t=1,49 \mathrm{~s}$ \\
\hline 9 & 22 & 79,2 & & \\
\hline
\end{tabular}

Unkritisch

Kritisch

Kippen

$s=$ Spurversatz

$t=$ Zeit bis zum

Radlastverlust

Abb. 5-29: Ergebnisse des 12 t-Gliederzugs für eine Fahrzeuggeschwindigkeit von 85 km/h 
In Abb. 5-30 ist die Fahrdynamik im unbeladenen Zustand für eine Windgeschwindigkeit von $15,5 \mathrm{~ms}(56 \mathrm{~km} / \mathrm{h})$ dargestellt, d.h. für die Windlast, bei der es in der Simulation zum ersten Mal zu einem Kippen des Anhängers kommt. Entsprechend lange fällt die zugehörige Zeitspanne aus.

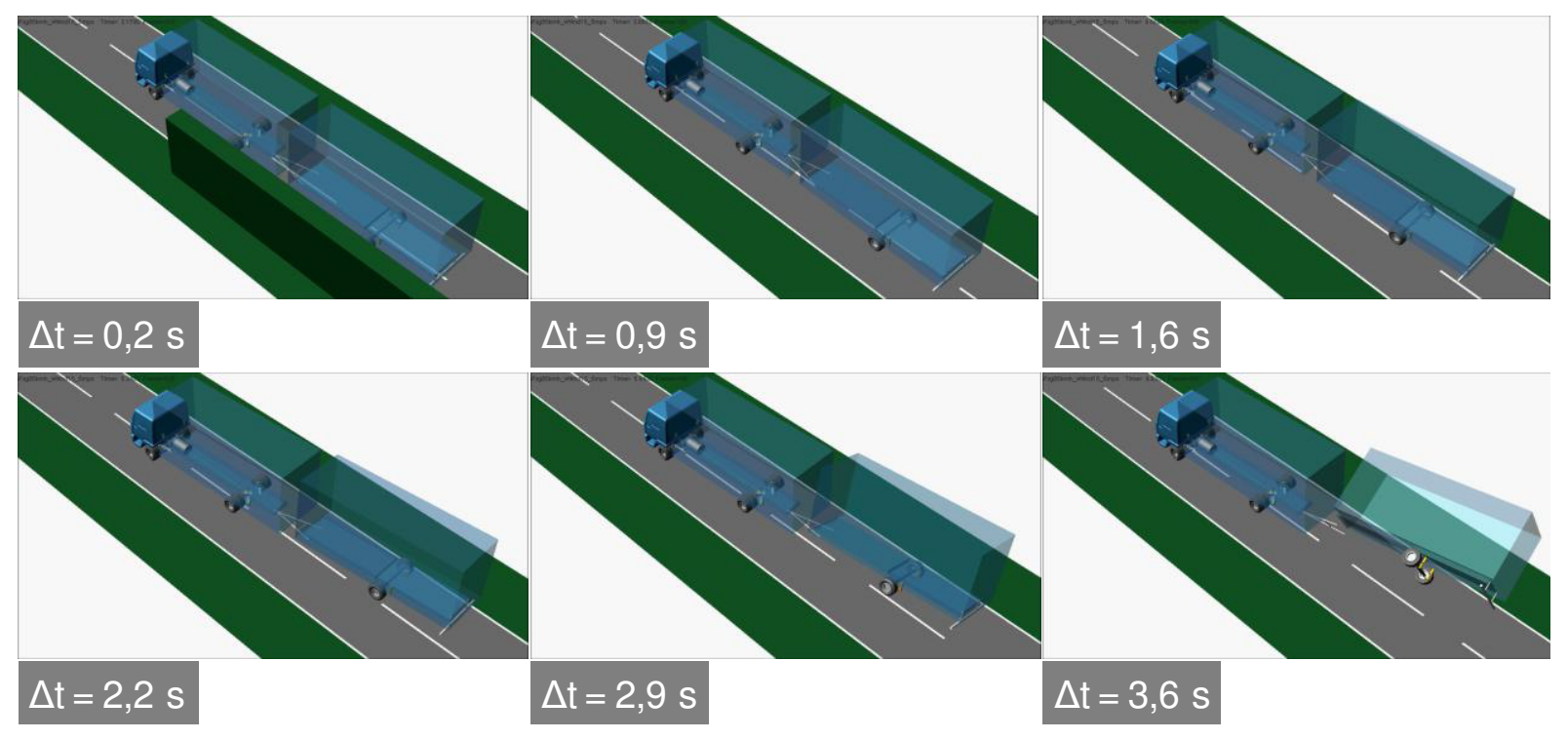

Abb. 5-30: Unbeladener $12 \mathrm{t}-\mathrm{Zug}\left(\mathrm{V}_{\mathrm{Fzg}}=85 \mathrm{~km} / \mathrm{h}\right.$, $\left.\mathrm{V}_{\text {Wind_aufbauend }}=15,5 \mathrm{~m} / \mathrm{s}(56 \mathrm{~km} / \mathrm{h})\right)$

Im beladenen Zustand erhöht sich die Zeitspanne bis zum Kippen des Anhängers nochmals um ca. 0,8 s, wie aus Abb. 5-31 hervorgeht. Die zugrundeliegende Windgeschwindigkeit von $20,5 \mathrm{~m} / \mathrm{s}(74 \mathrm{~km} / \mathrm{h})$ markiert wiederum die Windlast, ab der erstmals ein Kippen des Anhängers erfolgt. Im Windgeschwindigkeitsbereich oberhalb von $16 \mathrm{~m} / \mathrm{s}(58 \mathrm{~km} / \mathrm{h}) \mathrm{kommt}$ es vorher bereits zu kritischen Spurabweichungen, welche etwas geringer ausfallen als im unbeladenen Zustand.

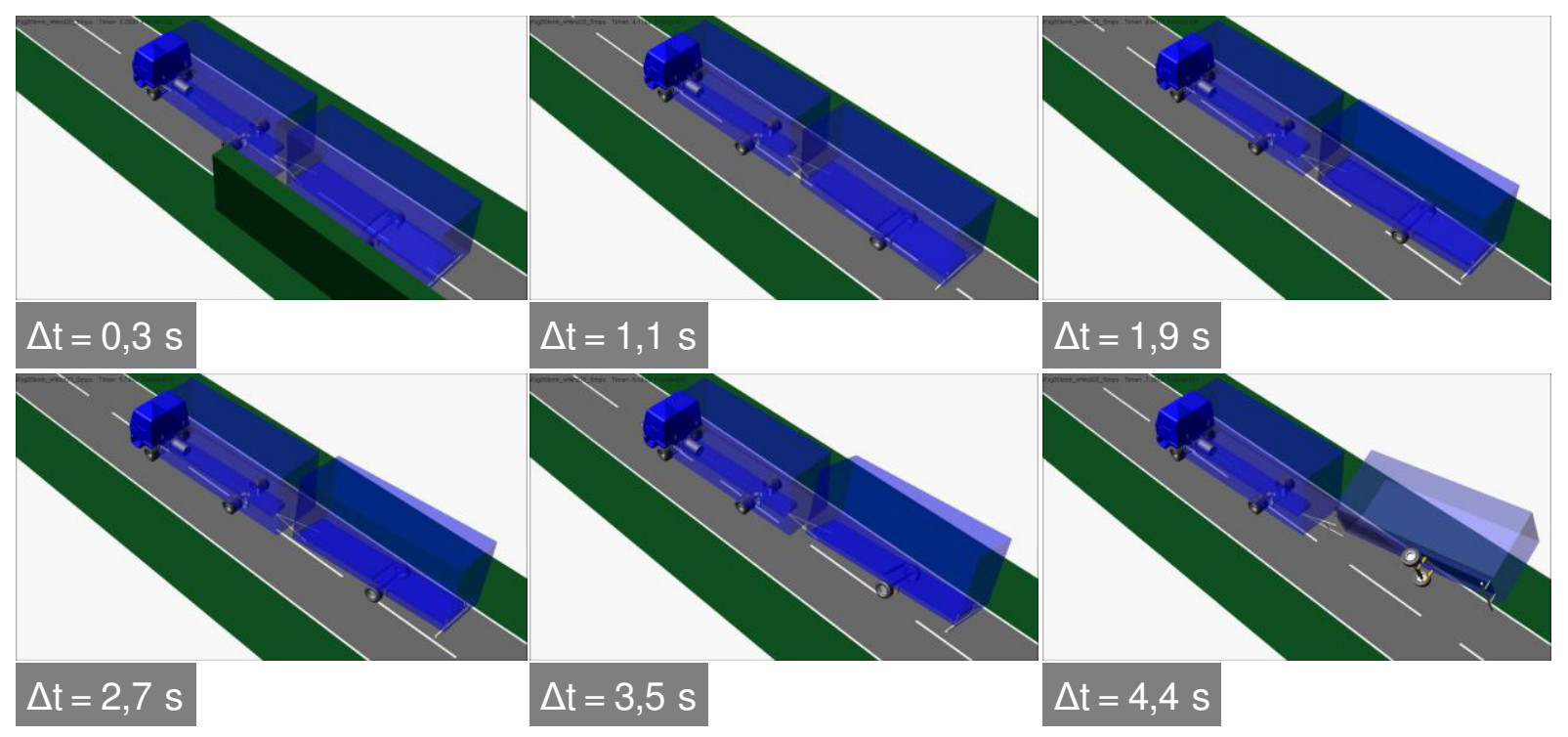

Abb. 5-31: Beladener $12 \mathrm{t}-\mathrm{Zug}\left(\mathrm{v}_{\mathrm{Fzg}}=85 \mathrm{~km} / \mathrm{h}\right.$, $\left.\mathrm{V}_{\text {Wind_aufbauend }}=20,5 \mathrm{~m} / \mathrm{s}(74 \mathrm{~km} / \mathrm{h})\right)$ 


\subsubsection{Implementierung von Sturmstützen}

Zur Untersuchung der in Kapitel 2.2 vorgestellten Sturmstützen für 12 t-Leicht-Lkw-Kombinationen werden diese, wie in Kapitel 5.3 beschrieben, auf Basis entsprechender Konstruktionsdaten in das Simulationsmodell implementiert (Abb. 5-32).

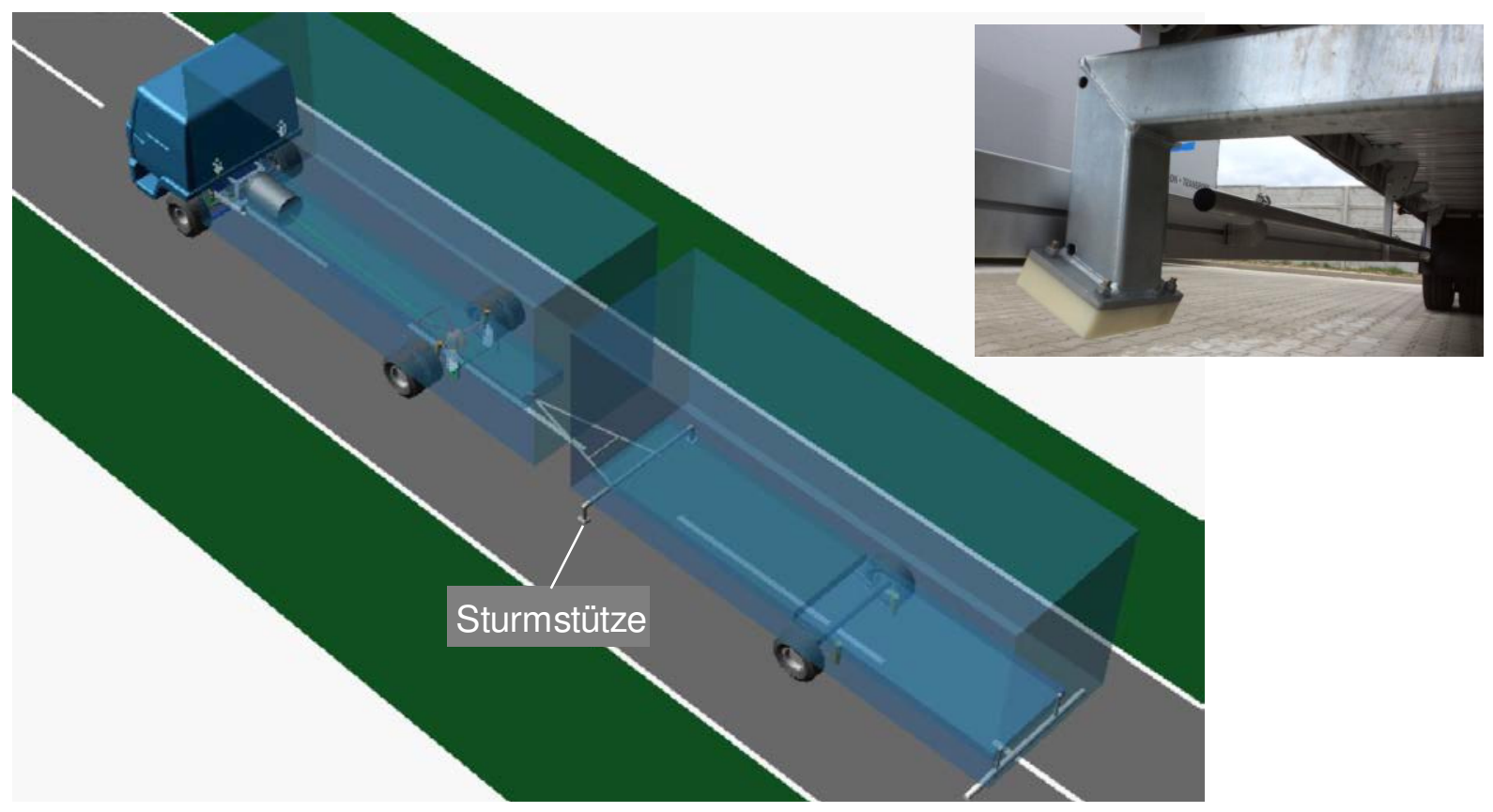

Abb. 5-32: Implementierung von Sturmstützen in das Leicht-Lkw-Simulationsmodell

Als Referenz dient der leere Beladungszustand bei direktem Wind. Für diese Konstellation kippt der Anhänger trotz Sturmstützen bei gleicher kritischer Windgeschwindigkeit $(16 \mathrm{~m} / \mathrm{s})$ wie zuvor in Kapitel 5.5.1.2 ohne Sturmstützen. Allerdings wird das Zugfahrzeug dabei regelrecht ausgehebelt, sodass die Hinterachse den Kontakt zur Fahrbahn verliert (Abb. 5-33).

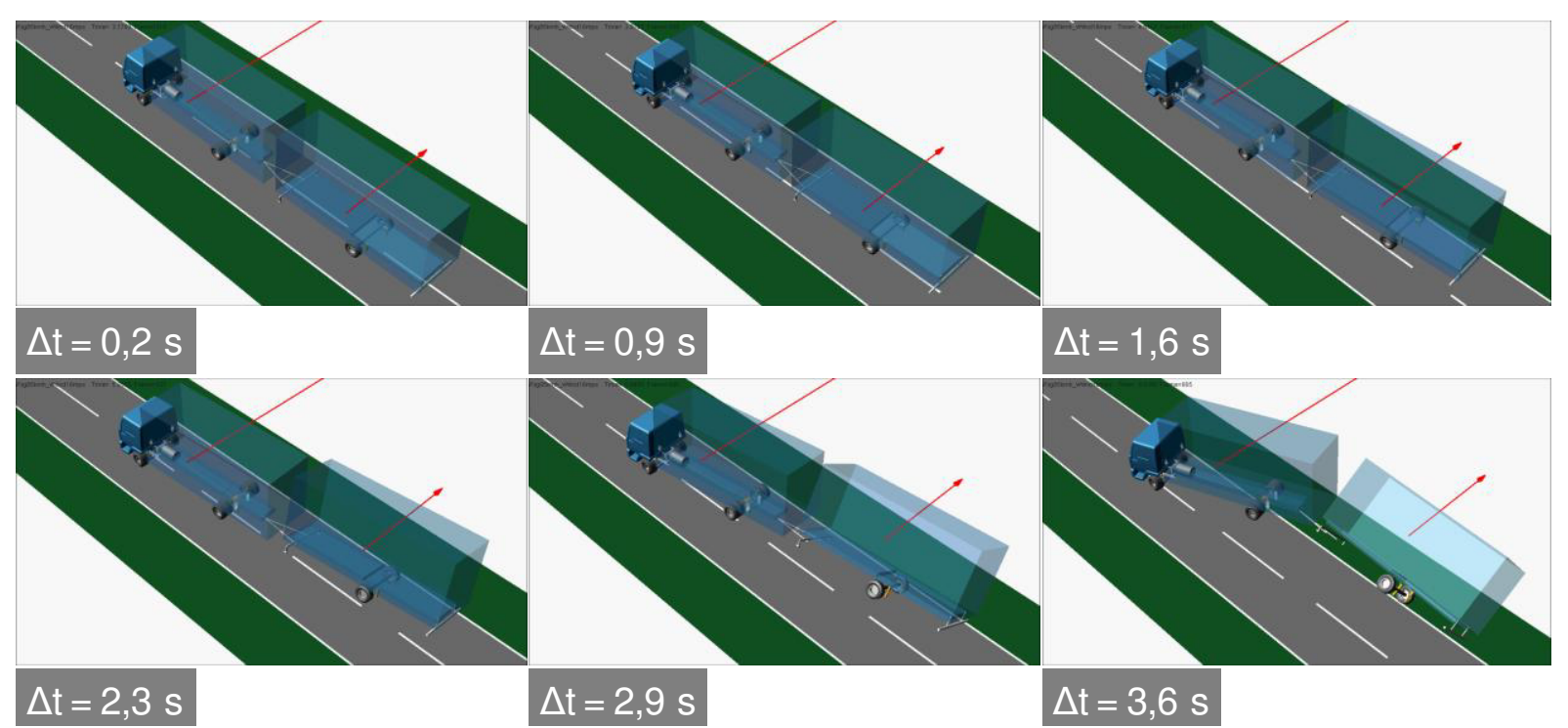

Abb. 5-33: Unbel. $12 \mathrm{t}-$ Zug mit Sturmstützen $\left(\mathrm{v}_{\mathrm{Fzg}}=85 \mathrm{~km} / \mathrm{h}, \mathrm{v}_{\text {Wind_direkt }}=16 \mathrm{~m} / \mathrm{s}(58 \mathrm{~km} / \mathrm{h})\right)$ 
Aufgrund des Einflusses der Sturmstützen fällt die Zeitspanne bis zum Kippen um ca. $1 \mathrm{~s}$ länger aus. Durch den konstant angreifenden Wind wird der Anhänger einfach über die jeweilige Stütze hinweg gekippt. Anders verhält es sich, wenn man den Windangriff auf eine Dauer von drei Sekunden beschränkt. In diesem Falle kommt es für die Standard-LeichtLkw-Kombination nachwievor zu einem Kippen des Anhängers, während durch Implementierung von Sturmstützen ein Kippen verhindert werden kann (Abb. 5-34 \& Abb. 5-35). Allerdings kommt es auch hier zu einem Aushebeln der Zugmaschine durch den gestützten Anhänger und damit zu einer fahrdynamisch höchst instabilen Situation, sodass nachwievor von einem erheblichen Unfallrisiko auszugehen ist. Abb. 5-37 zeigt den Einfluss der Sturmstütze auf die Fahrdynamik nochmals aus der Frontperspektive. Im dritten Bild ist zu erkennen, dass das Zugfahrzeug hinten angehoben wird und die Hinterachse dadurch frei in der Luft schwebt.

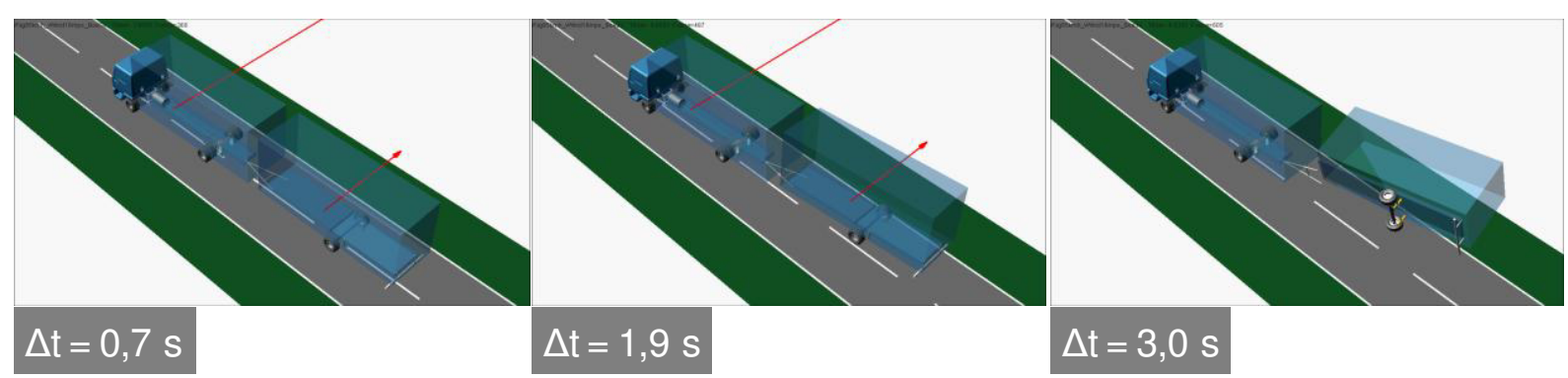

Abb. 5-34: Unbeladener $12 \mathrm{t}$-Zug ohne Sturmstützen $\left(\mathrm{v}_{\mathrm{Fzg}}=85 \mathrm{~km} / \mathrm{h}\right.$, $\mathrm{V}_{\text {Wind_direkt }}=16 \mathrm{~m} / \mathrm{s}$ $\left.(58 \mathrm{~km} / \mathrm{h}), \Delta \mathrm{t}_{\text {Windlast }}=3 \mathrm{~s}\right)$
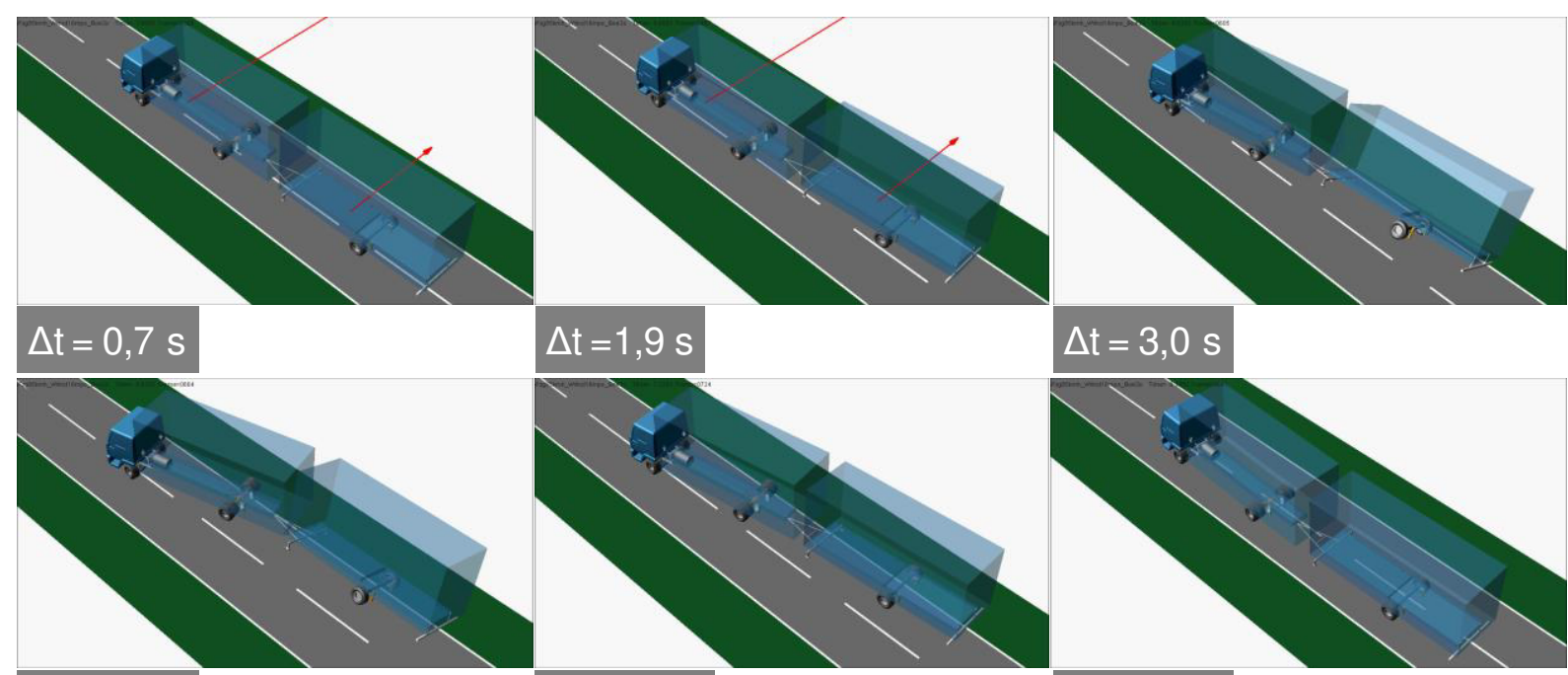

$\Delta \mathrm{t}=3,6 \mathrm{~s}$

$\Delta \mathrm{t}=4,2 \mathrm{~s}$

$\Delta t=5,4 s$

Abb. 5-35: Unbeladener $12 \mathrm{t}$-Zug mit Sturmstützen $\left(\mathrm{v}_{\mathrm{Fzg}}=85 \mathrm{~km} / \mathrm{h}\right.$, $\mathrm{v}_{\text {Wind_direkt }}=16 \mathrm{~m} / \mathrm{s}$ $\left.(58 \mathrm{~km} / \mathrm{h}), \Delta \mathrm{t}_{\text {Windlast }}=3 \mathrm{~s}\right)$ - kein Kippen des Anhängers

Generell ist die Dauer der Windböe von entscheidender Bedeutung. Wie aus Abb. 5-36 hervorgeht, sind die Sturmstützen nur bei kurzen Böen im Bereich von einer Sekunde wirklich wirksam. Dauert die Böe länger, ergibt sich kaum eine Minderung der Kippgefahr. 


\begin{tabular}{|c|c|c|c|c|c|c|c|c|}
\hline \multicolumn{3}{|c|}{$V_{\text {Fahrzeug }}=85 \mathrm{~km} / \mathrm{h}$} & \multicolumn{6}{|c|}{ Direkte Windböe } \\
\hline \multirow{2}{*}{$\begin{array}{l}\text { Wind- } \\
\text { stärke }\end{array}$} & \multicolumn{2}{|c|}{ VWind } & \multicolumn{3}{|c|}{ Ohne Sturmstützen } & \multicolumn{3}{|c|}{ Mit Sturmstützen } \\
\hline & {$[\mathrm{m} / \mathrm{s}]$} & {$[\mathrm{km} / \mathrm{h}]$} & $\Delta t=1 \mathrm{~s}$ & $\Delta t=2 s$ & $\Delta t=3 \mathrm{~s}$ & $\Delta t=1 \mathrm{~s}$ & $\Delta t=2 s$ & $\Delta \mathrm{t}=3 \mathrm{~s}$ \\
\hline 7 & 16 & 57,6 & & & & & & \\
\hline 7 & 17 & 61,2 & & & & & & \\
\hline 8 & 18 & 64,8 & & & & & & \\
\hline 8 & 19 & 68,4 & & & & & & \\
\hline 8 & 20 & 72 & & & & & & \\
\hline 9 & 21 & 75,6 & & & & & & \\
\hline
\end{tabular}

Abb. 5-36: Ergebnisse des 12 t-Gliederzugs für verschiedene Dauern der direkten Windböe
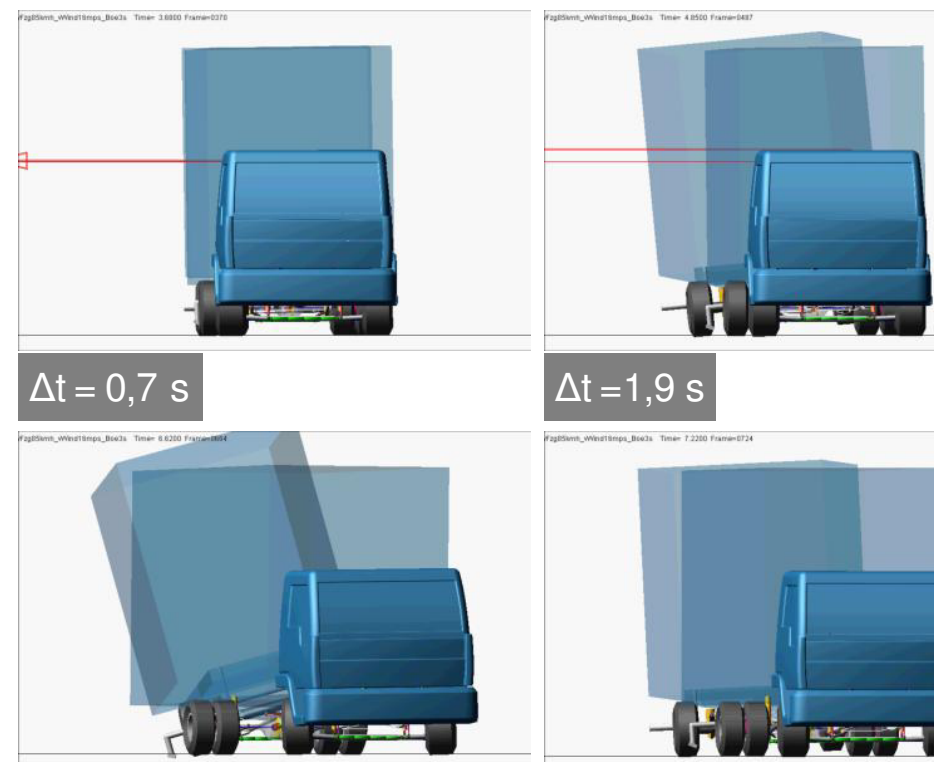

$\Delta t=3,6 s$

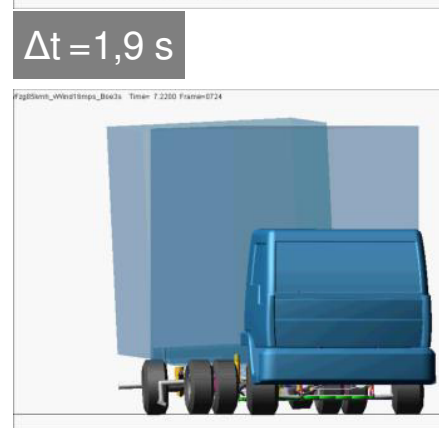

$\Delta t=4,2 s$

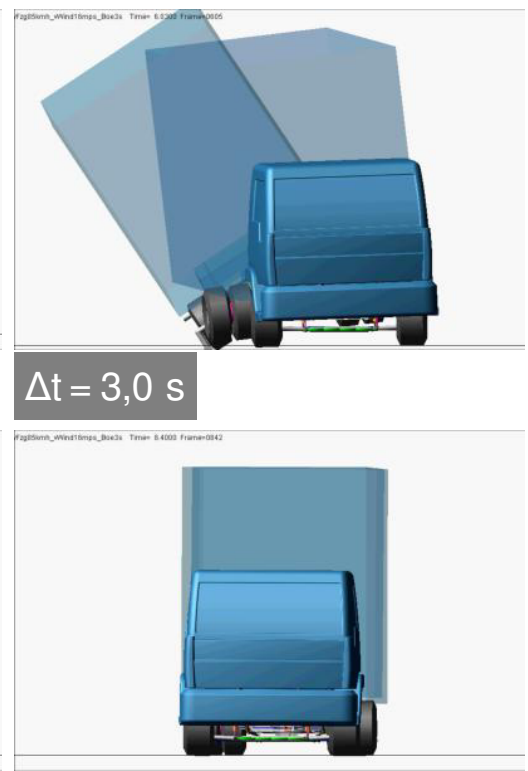

$\Delta t=5,4 \mathrm{~s}$

Abb. 5-37: Unbeladener $12 \mathrm{t}$-Zug mit Sturmstützen $\left(\mathrm{v}_{\mathrm{Fzg}}=85 \mathrm{~km} / \mathrm{h}\right.$, $\mathrm{v}_{\text {Wind_direkt }}=16 \mathrm{~m} / \mathrm{s}$ $\left.(58 \mathrm{~km} / \mathrm{h}), \Delta \mathrm{t}_{\text {Windlast }}=3 \mathrm{~s}\right)$ - kein Kippen des Anhängers (Frontansicht)

\subsubsection{Parametervariationen}

Zur Absicherung der Simulationsergebnisse und zur Untersuchung weiterer Aspekte im Zusammenhang mit Leicht-Lkw-Kombinationen werden verschiedene Parametervariationen sowie Modellanpassungen im Rahmen der Simulationen berücksichtigt. Entsprechende Simulationen im Vorfeld der eigentlichen Hauptuntersuchungen haben gezeigt, dass die Reibung in der Deichsel, die Federsteifigkeit der Achse und der Reibwert der Straße keine nennenswerte Auswirkung auf das Kippverhalten des Anhängers haben. Im Folgenden werden zunächst mögliche fahrzeugmodellseitige Einflüsse, wie die Fahrzeuggeschwindigkeit, das Fahrerreglermodell, ein ungleicher Beladungszustand von Zugfahrzeug und An- 
hänger, eine zweite Anhängerachse sowie eine Auflastung des Anhängers, untersucht. Des Weiteren erfolgen in Bezug auf das Seitenwindmodell Simulationen hinsichtlich der Frequenz der Windlast, der Fahrdynamik bei überkritischen Windgeschwindigkeiten, einer Schräganströmung des Leicht-Lkw-Gespanns sowie eines Anhängers mit geöffneter Plane, d.h. ohne Windangriffsfläche. Auch der Einsatz von Ballasttanks bei Leerfahrten wird betrachtet.

\subsubsection{Fahrzeuggeschwindigkeit}

Die Fahrzeuggeschwindigkeit hat einen signifikanten Einfluss auf die sich unter Seitenwindeinfluss einstellenden Spurabweichungen. Je höher die Fahrzeuggeschwindigkeit, desto mehr Längskraft muss an den Reifen aufgebracht werden, wodurch gleichzeitig das Querkraftpotential abnimmt, d.h. es steht weniger Seitenkraft zur Kompensation der Windlast zur Verfügung. Somit nehmen die Spurabweichungen mit der Fahrzeuggeschwindigkeit zwangsläufig zu, wie auch die in Abb. 5-38 dargestellten Simulationsergebnisse für den unbeladenen $12 \mathrm{t}$-Gliederzug zeigen. Die hier definierte Windgeschwindigkeit von $15 \mathrm{~m} / \mathrm{s}(54 \mathrm{~km} / \mathrm{h})$, welche nach $3 \mathrm{~s}$ einsetzt, liegt knapp unterhalb der Kippgrenze, sodass kein Kippen auftritt. Allerdings sind Spurabweichungen von einem halben Meter und mehr generell als kritisch anzusehen, was für die vorliegende Windgeschwindigkeit bereits bei einer geringen Fahrzeuggeschwindigkeit von $30 \mathrm{~km} / \mathrm{h}$ der Fall ist. Auf die Kippgrenze selbst hat die Fahrzeuggeschwindigkeit keinen Einfluss. Auch das stehende Gespann ( $\left.\mathrm{v}_{\mathrm{Fzg}}=0 \mathrm{~km} / \mathrm{h}\right) \mathrm{kippt}$ im unbeladenen Zustand bei einer Windgeschwindigkeit von $16 \mathrm{~m} / \mathrm{s}$ (direkter Wind). Lediglich die Zeitspanne bis zum Kippen des Anhängers fällt etwas mehr als eine Sekunde länger aus.

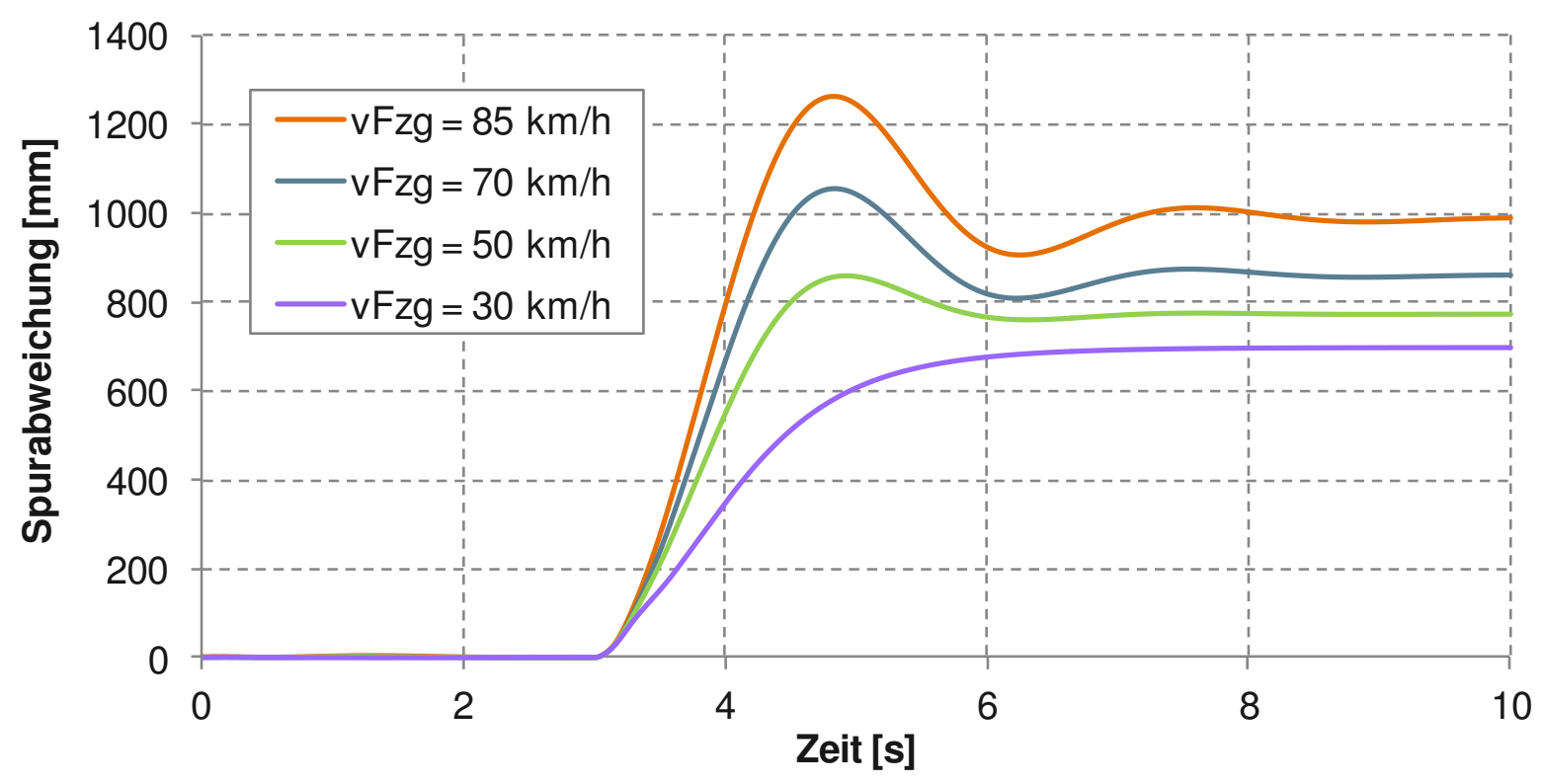

Abb. 5-38: Spurabweichung beim unbeladenen $12 \mathrm{t}$-Zug für verschiedene Fahrzeuggeschwindigkeiten $\left(v_{\text {Wind_direkt }}=15 \mathrm{~m} / \mathrm{s}(54 \mathrm{~km} / \mathrm{h})\right)$

Da die im Rahmen der durchgeführten Simulationen zugrunde gelegte Fahrzeuggeschwindigkeit von $85 \mathrm{~km} / \mathrm{h}$ das obere Spektrum für Lkw repräsentiert, wird hier entsprechend auch der fahrdynamisch möglichst kritischste Zustand abgebildet. 


\subsubsection{Einfluss des Fahrerreglers}

Das für die Fahrzeugmodelle verwendete Standard-Adams/Car-Fahrermodell repräsentiert einen gut reagierenden Fahrer. Um dessen Einfluss auf die Ergebnisse zur Kippstabilität bewerten zu können, sind für die unbeladene 12 t-Leicht-Lkw-Kombination verschiedene Vergleichssimulationen mit einem ungeregelten Modell durchgeführt worden. Dabei wird das Lenkrad konstant auf einem Lenkwinkel von $0^{\circ}$ gehalten, wodurch sich deutlich höhere Spurabweichungen einstellen und das Gespann schneller instabil wird. In Abb. 5-39 ist dies beispielhaft für eine Windgeschwindigkeit von $15,5 \mathrm{~m} / \mathrm{s}$, also nahe der Kippgrenze des geregelten Modells (Abb. 5-23), dargestellt. Im Gegensatz zum geregelten Modell kommt es hier aber bereits zum Kippen des Anhängers, welches zudem ca. 0,5 s schneller erfolgt. Generell kippt das ungeregelte Modell ab einer Windgeschwindigkeit von $14 \mathrm{~m} / \mathrm{s}$ (50 km/h), was einer Absenkung der Kippgrenze um 2 m/s entspricht.

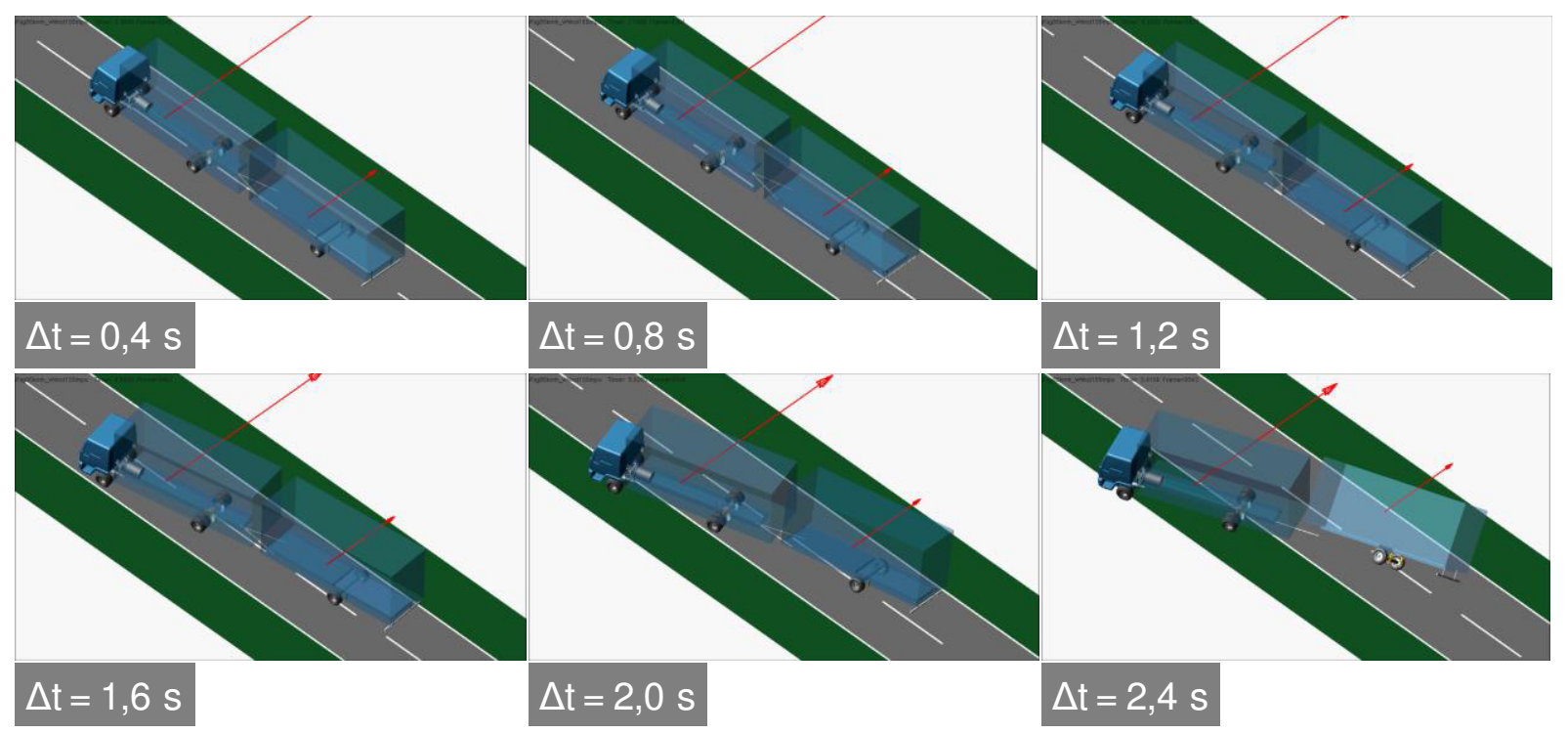

Abb. 5-39: Unbel. $12 \mathrm{t}$-Zug ohne Fahrerregler $\left(\mathrm{v}_{\mathrm{Fzg}}=85 \mathrm{~km} / \mathrm{h}\right.$, $\left.\mathrm{v}_{\text {Wind_direkt }}=15,5 \mathrm{~m} / \mathrm{s}(56 \mathrm{~km} / \mathrm{h})\right)$

Aus Abb. 5-40 geht die Fahrdynamik bei einer Windgeschwindigkeit deutlich jenseits der Kippgrenze hervor. Bei dem hier definierten Wert von $20 \mathrm{~m} / \mathrm{s}$ ist die Zeitspanne bis zum Kippen des Anhängers nochmals um ca. $1 \mathrm{~s}$ kürzer. Auch besteht hier zeitlich kaum noch ein Unterschied zum geregelten Modell.

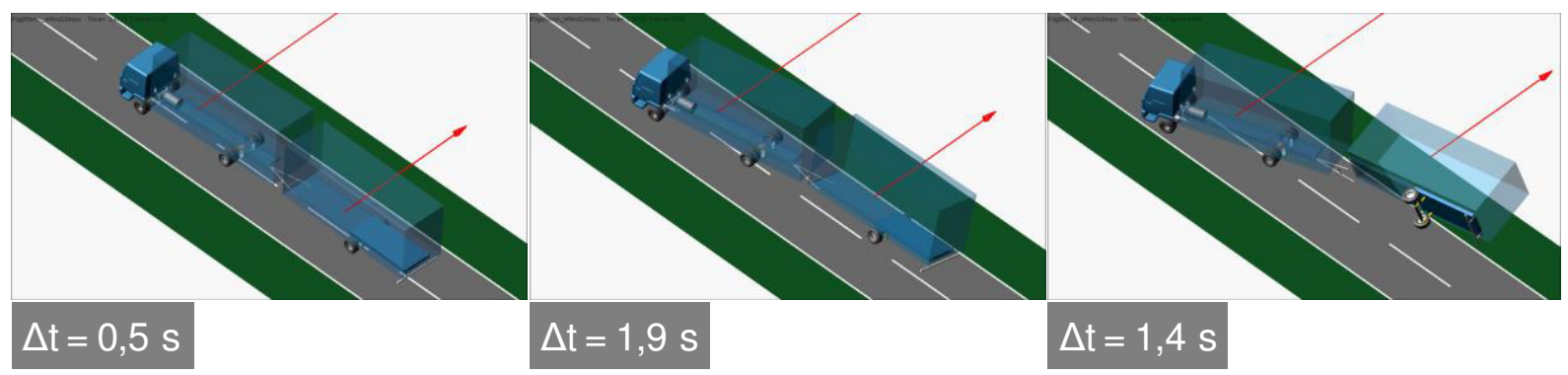

Abb. 5-40: Unbel. $12 \mathrm{t}$-Zug ohne Fahrerregler $\left(\mathrm{v}_{\mathrm{Fzg}}=85 \mathrm{~km} / \mathrm{h}\right.$, $\left.\mathrm{v}_{\text {Wind_direkt }}=20 \mathrm{~m} / \mathrm{s}(72 \mathrm{~km} / \mathrm{h})\right)$ 


\subsubsection{Ungleicher Beladungszustand von Zugfahrzeug und Anhänger}

Während bislang ausschließlich der unbeladene und der vollbeladene Zustand betrachtet worden sind, werden für das Leicht-Lkw-Gespann im Folgenden zwei weitere Beladungszustände, nämlich der eines vollbeladenen Zugfahrzeugs mit unbeladenem Anhänger sowie der umgekehrte Fall eines unbeladenen Zugfahrzeugs mit vollbeladenem Anhänger, untersucht. Bei beladenem Zugfahrzeug und unbeladenem Anhänger ergeben sich im Vergleich zum komplett leeren Gespann kaum Unterschiede. Der Anhänger verhält sich hier ähnlich kritisch. Stellt man den Fall eines unbeladenen Zugfahrzeugs mit beladenem Anhänger dem vollbeladenen Zustand des Gespanns gegenüber, wird ersichtlich, dass als Folge der deutlich höheren Spurabweichung des leeren Zugfahrzeugs das Kippen des Anhängers bei geringerer Windstärke erfolgt (Abb. 5-41 \& Abb. 5-42). So kommt es in dieser Konstellation bereits ab einer Windgeschwindigkeit von 20 m/s (72 km/h) zum Kippen des vollbeladenen Anhängers, was eine Windstärke niedriger einzustufen ist als die kritische Windgeschwindigkeit von $21,5 \mathrm{~m} / \mathrm{s}$ (77 km/h) beim vollbeladenen Gespann (Abb. 5-25). Im Vergleich zum komplett leeren Gespann, wo der Anhänger bereits bei einer Windgeschwindigkeit von 58 km/h kippt, nimmt die Windanfälligkeit aufgrund der erhöhten Anhängermasse allerdings deutlich ab.

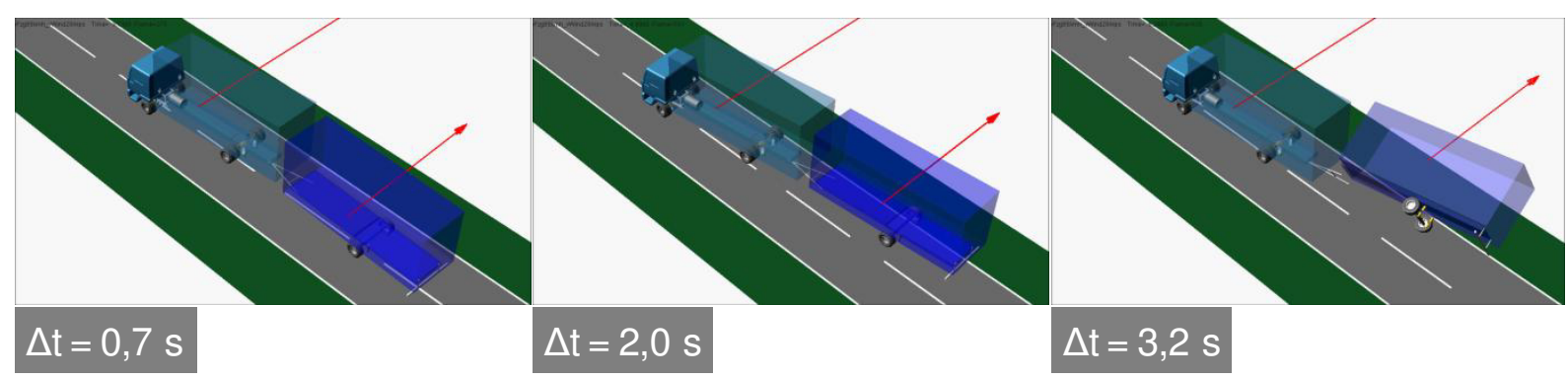

Abb. 5-41: $12 \mathrm{t}$-Zug mit unbeladenem Zugfahrzeug und beladenem Anhänger $\left(\mathrm{v}_{\mathrm{Fzg}}=\right.$ $\left.85 \mathrm{~km} / \mathrm{h}, \mathrm{V}_{\text {Wind_direkt }}=20 \mathrm{~m} / \mathrm{s}(72 \mathrm{~km} / \mathrm{h})\right)$ - Kippen des Anhängers

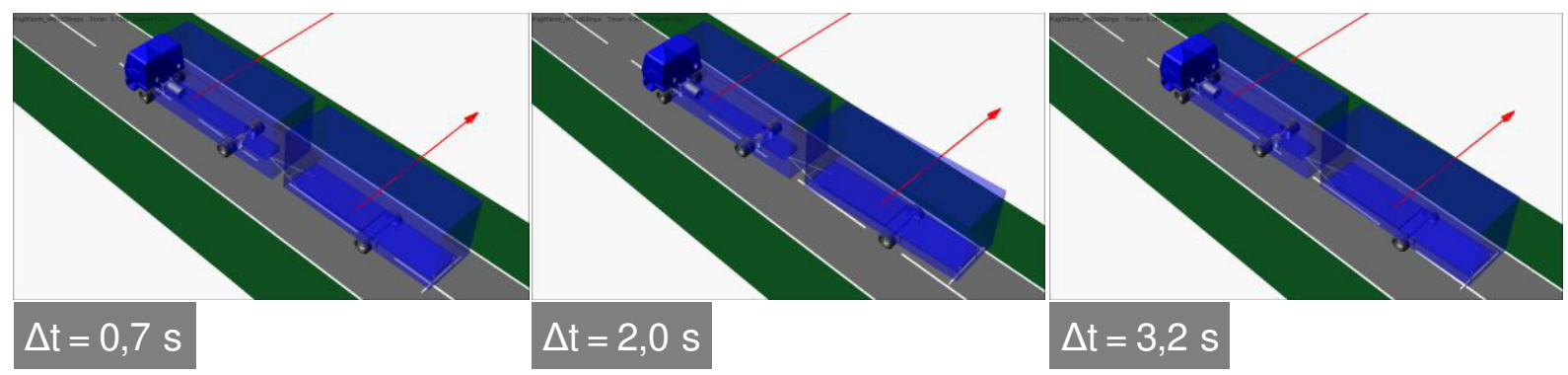

Abb. 5-42: Komplett beladener $12 \mathrm{t}-\mathrm{Zug}\left(\mathrm{v}_{\mathrm{Fzg}}=85 \mathrm{~km} / \mathrm{h}\right.$, $\left.\mathrm{v}_{\text {Wind_direkt }}=20 \mathrm{~m} / \mathrm{s}(72 \mathrm{~km} / \mathrm{h})\right)-$ Kein Kippen des Anhängers

\subsubsection{Implementierung einer zweiten Anhängerachse beim 12 t-Gliederzug}

Die Implementierung einer zweiten zentralen Anhängerachse beim $12 \mathrm{t}$-Gliederzug führt zu einem Mehrgewicht von ca. $200 \mathrm{~kg}$. Eine entsprechende Simulation mit der für den leeren 
$12 \mathrm{t}$-Zug in Bezug auf Kippen kritischen Windgeschwindigkeit von $16 \mathrm{~m} / \mathrm{s}$ ( $58 \mathrm{~km} / \mathrm{h})$ offenbart keine Minderung der Kippgefahr (Abb. 5-43). Das Kippen tritt im Vergleich zum unbeladenen Standardzug (Abb. 5-24) lediglich etwa 1 Sekunde später ein.

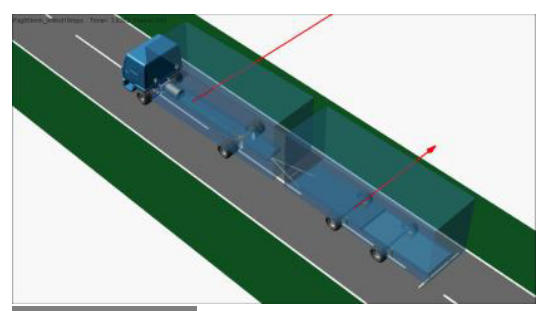

$\Delta t=0,8 \mathrm{~s}$

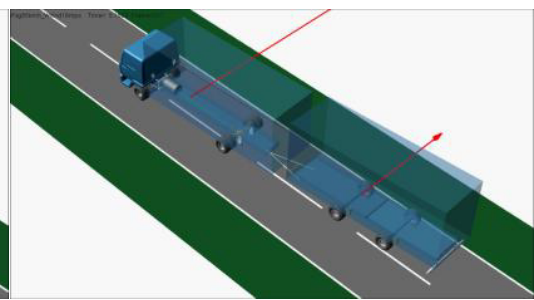

$\Delta t=2,2 \mathrm{~s}$

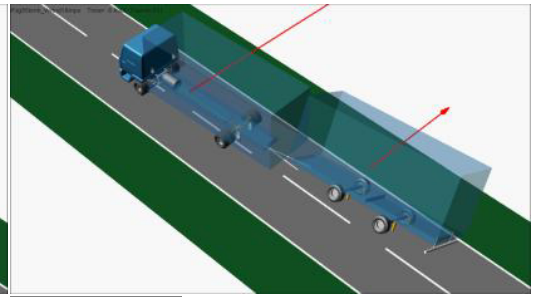

$\Delta t=3,5 s$

Abb. 5-43: Unbeladener $12 \mathrm{t}$-Zug mit Doppelachse $\left(\mathrm{v}_{\mathrm{Fzg}}=85 \mathrm{~km} / \mathrm{h}\right.$, $\mathrm{v}_{\text {Wind_direkt }}=16 \mathrm{~m} / \mathrm{s}$ $(58 \mathrm{~km} / \mathrm{h}))$ - Kippen des Anhängers

\subsubsection{Auflastung des Anhängers beim $12 \mathrm{t}$-Gliederzug}

Entsprechend der Ausführungen in Kapitel 2.2 wird der Einfluss einer Auflastung des Anhängers um 2 t ebenfalls simulativ betrachtet. Im Zuge der Auflastung ergeben sich für den Anhänger entsprechend höhere Radlasten und eine höhere Trägheit, wodurch sich die Windanfälligkeit bzw. die Kippgefahr etwas reduzieren sollte. Dies wird durch die Simulationsergebnisse bestätigt. Für den vollbeladenen $12 \mathrm{t}$-Gliederzug mit aufgelastetem Anhänger, also einem 14 t-Gliederzug (Abb. 5-44), setzt das Kippen im Vergleich zum vollbeladenen Standardzug (Abb. 5-25) bei einer um 2,5 m/s höheren Windgeschwindigkeit ein, d.h. bei $24 \mathrm{~m} / \mathrm{s}$ $(86 \mathrm{~km} / \mathrm{h})$ statt $21,5 \mathrm{~m} / \mathrm{s}(77 \mathrm{~km} / \mathrm{h})$. Zudem erfolgt das Kippen mehr als eine halbe Sekunde später. Im leeren Zustand bleibt das Fahrzeug allerdings unverändert kritisch.

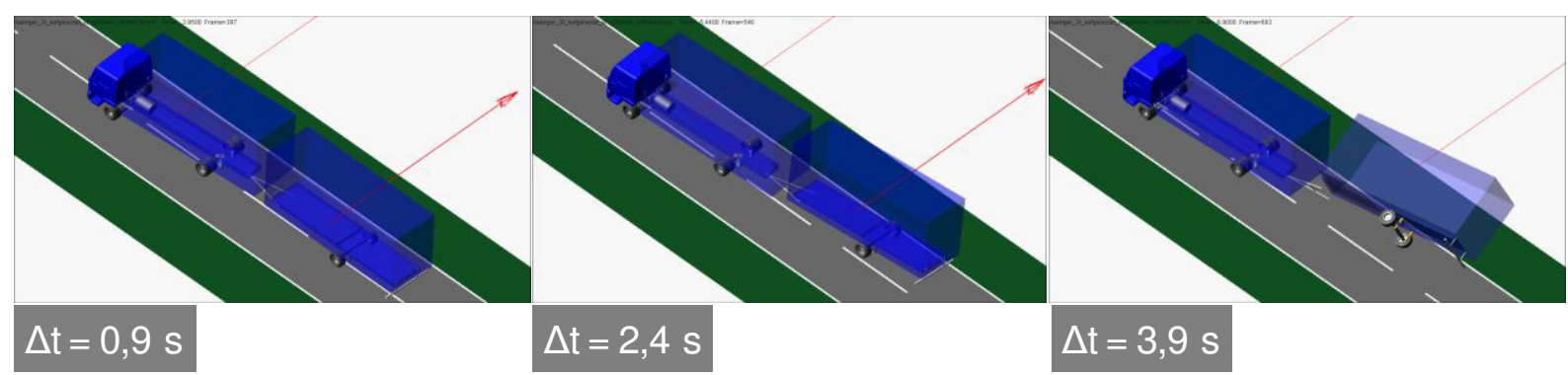

Abb. 5-44: $14 \mathrm{t}$-Gliederzug: Beladener $12 \mathrm{t}$-Zug mit um $2 \mathrm{t}$ aufgelastetem Anhänger $\left(\mathrm{v}_{\mathrm{Fzg}}=\right.$ $\left.85 \mathrm{~km} / \mathrm{h}, \mathrm{v}_{\text {Wind_direkt }}=24 \mathrm{~m} / \mathrm{s}(86 \mathrm{~km} / \mathrm{h})\right)-$ Kippen des Anhängers

\subsubsection{Alternierender Wind}

Bei sinusförmiger Aufbringung des Windes $\left(0 \mathrm{~m} / \mathrm{s}\right.$ bis $\left.v_{\text {Wind }}\right)$ verschiebt sich die kritische Grenze nach oben. In Bezug auf das Frequenzband, welches dabei von 0,1 bis $10 \mathrm{~Hz}$ in der Simulation durchlaufen wird (Kapitel 5.2), ist in erster Linie der niedrige Frequenzbereich kritisch. Das obere Frequenzspektrum ist aufgrund der Trägheit der Fahrzeugmodelle weniger relevant. Der 40 t-Gliederzug verhält sich für das alternierende Windmodell im berücksich- 
tigten Windgeschwindigkeitsspektrum komplett unkritisch, d.h. auch im unbeladenen Zustand bleiben die Spurabweichungen unterhalb von einem halben Meter. Beim $12 \mathrm{t}$-Gliederzug kommt es für den beladenen Zustand ebenfalls zu keinem Kippen bis zur maximalen Windgeschwindigkeit von $144 \mathrm{~km} / \mathrm{h}$. Auch sind die Spurabweichungen im Vergleich zu den Windmodellen „direkter Wind“ (Kapitel 5.5.1.2) und „aufbauender Wind“ (Kapitel 5.5.2.2) deutlich geringer. Im leeren Zustand kommt es erst ab einer Windgeschwindigkeit von $24 \mathrm{~m} / \mathrm{s}$ (86 km/h) zum Kippen des Anhängers (Abb. 5-45). Die Zeitspanne bis zum Kippen beträgt dabei etwa $2 \mathrm{~s}$. Somit hat die zeitliche Ausprägung der Windlast entscheidende Bedeutung.

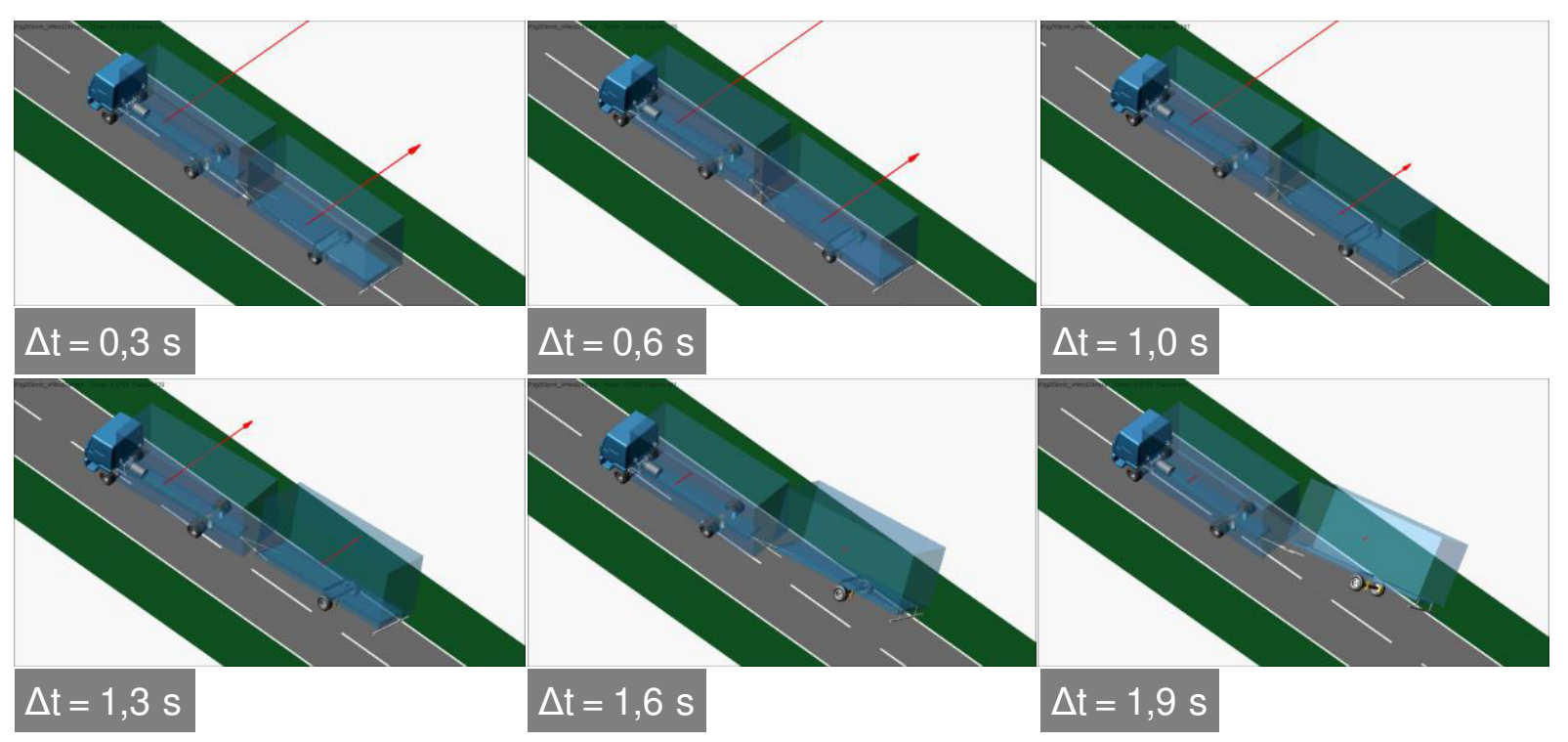

Abb. 5-45: Unbeladener $12 \mathrm{t}-\mathrm{Zug}\left(\mathrm{v}_{\mathrm{Fzg}}=85 \mathrm{~km} / \mathrm{h}\right.$, $\left.\mathrm{v}_{\text {Wind_alternierend }}=24 \mathrm{~m} / \mathrm{s}(86 \mathrm{~km} / \mathrm{h})\right)$

\subsubsection{Einfluss überkritischer Windgeschwindigkeiten}

Betrachtet man die Fahrdynamik des 12 t-Gliederzuges bei Windstärken von 10 bis 12, also für schweren Sturm bis Orkan und Windgeschwindigkeiten über 88 km/h bzw. 118 km/h, so wird auch das Zugfahrzeug unmittelbar nach Einsetzen des Windes fahrdynamisch kritisch. Die Reifen verlieren dabei auf der windzugewandten Seite den Kontakt zum Boden. Für den unbeladenen Zustand ist dies bereits bei Sturm (Windstärke 9) der Fall. Abb. 5-46 zeigt exemplarisch die Fahrdynamik bei schwerem Sturm mit einer Windgeschwindigkeit von 25,5 m/s (92 km/h). Das Zugfahrzeug gerät durch die Windlast unmittelbar in Schräglage.

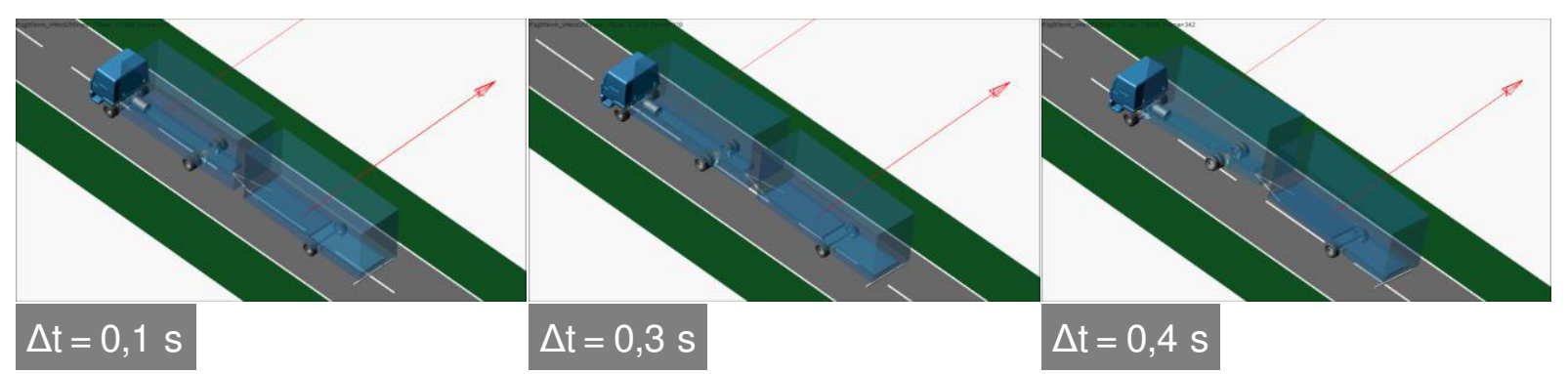

Abb. 5-46: Unbeladener $12 \mathrm{t}-$ Zug $\left(\mathrm{v}_{\mathrm{Fzg}}=85 \mathrm{~km} / \mathrm{h}\right.$, $\left.\mathrm{v}_{\text {Wind_direkt }}=25,5 \mathrm{~m} / \mathrm{s}(92 \mathrm{~km} / \mathrm{h})\right)$ 
Für den beladenen $12 \mathrm{t}$-Gliederzug ist dies erst bei Orkanstärke der Fall. Ab einer Windgeschwindigkeit von $36 \mathrm{~m} / \mathrm{s}$ (130 km/h) heben die windzugewandten Reifen der Zugmaschine unmittelbar nach Einsetzen des Windes vom Boden ab (Abb. 5-47). Aufgrund des damit verbundenen fahrdynamisch instabilen Zustands bricht die Simulation, wie auch in Abb. 5-46, frühzeitig ab und lässt ein mögliches vollständiges Kippen des Zugfahrzeugs offen.

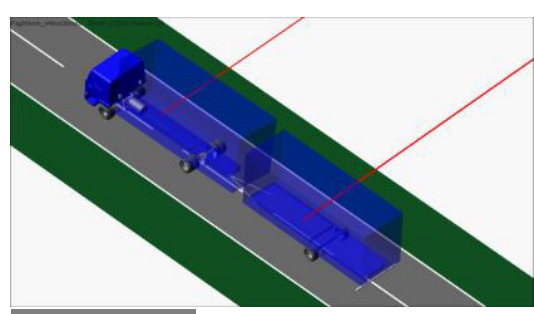

$\Delta \mathrm{t}=0,2 \mathrm{~s}$

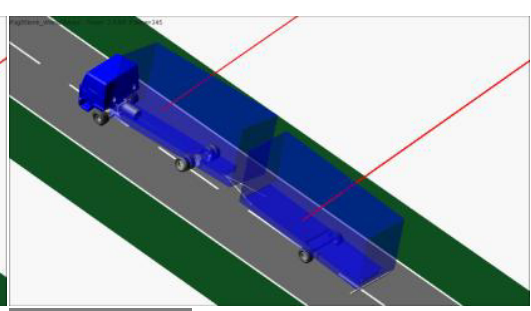

$\Delta t=0,4 s$

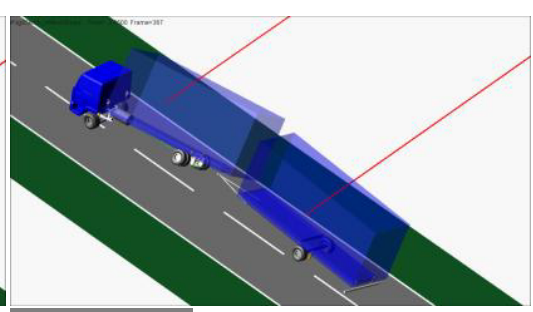

$\Delta t=0,7 \mathrm{~s}$

Abb. 5-47: Beladener $12 \mathrm{t}-\mathrm{Zug}\left(\mathrm{v}_{\mathrm{Fzg}}=85 \mathrm{~km} / \mathrm{h}\right.$, $\left.\mathrm{v}_{\text {Wind_direkt }}=36 \mathrm{~m} / \mathrm{s}(130 \mathrm{~km} / \mathrm{h})\right)$

\subsubsection{Schräganströmung}

Aufgrund der Ausführungen in Kapitel 5.2 ist im Rahmen der Simulationen auch ein schräg von vorne erfolgender Windangriff berücksichtigt worden. Der Winkel der Windkraft beträgt dabei $30^{\circ}$ zur Querrichtung. Hierzu wird der Windkraftvektor in eine quer- und eine längsgerichtete Komponente zerlegt, welche beide jeweils im Flächenschwerpunkt von Zugfahrzeug und Anhänger angreifen. Legt man die bei direktem Wind für den leeren $12 \mathrm{t}$-Gliederzug in Bezug auf Kippen kritische Windgeschwindigkeit von $16 \mathrm{~m} / \mathrm{s}$ (58 km/h) zugrunde (Abb. 5-24), so kommt es im Zuge der Schräganströmung des Gespanns zu keinem Kippen des Anhängers (Abb. 5-48). Dies erfolgt erst ab einer Windgeschwindigkeit von 17,5 m/s (63 km/h), wie in Abb. 5-49 dargestellt.
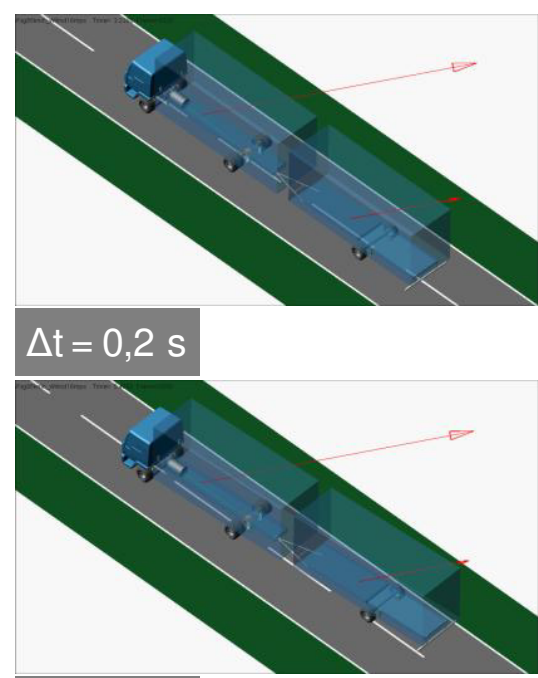

$\Delta t=2,5 s$

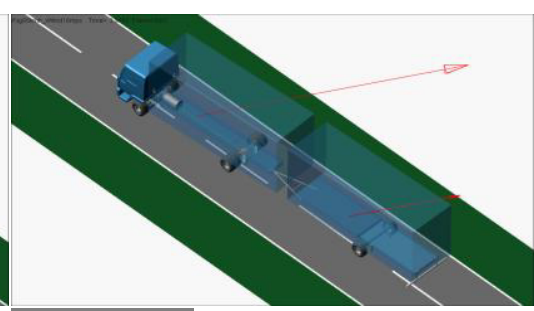

$\Delta \mathrm{t}=1,0 \mathrm{~s}$

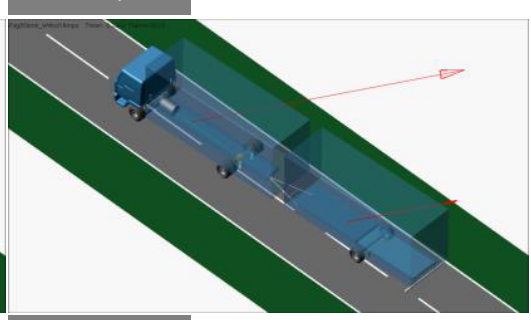

$\Delta t=3,2 s$

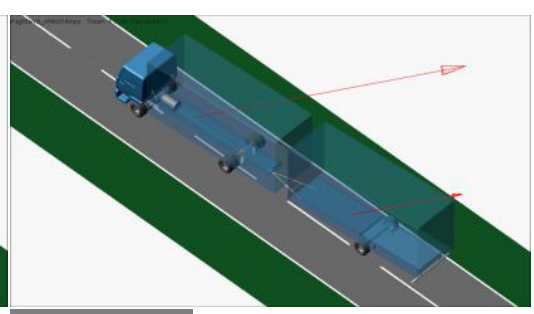

$\Delta \mathrm{t}=1,8 \mathrm{~s}$

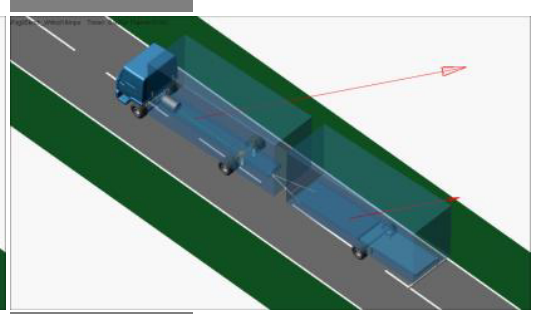

$\Delta \mathrm{t}=4,0 \mathrm{~s}$

Abb. 5-48: Unbeladener $12 \mathrm{t}$-Zug unter Schräganströmung $\left(v_{\mathrm{Fzg}}=85 \mathrm{~km} / \mathrm{h}\right.$, $\alpha_{\text {Wind }}=60^{\circ}\left(30^{\circ}\right.$ zur Querrichtung), $\left.v_{\text {Wind_direkt }}=16 \mathrm{~m} / \mathrm{s}(58 \mathrm{~km} / \mathrm{h})\right)$ - Kein Kippen des Anhängers 

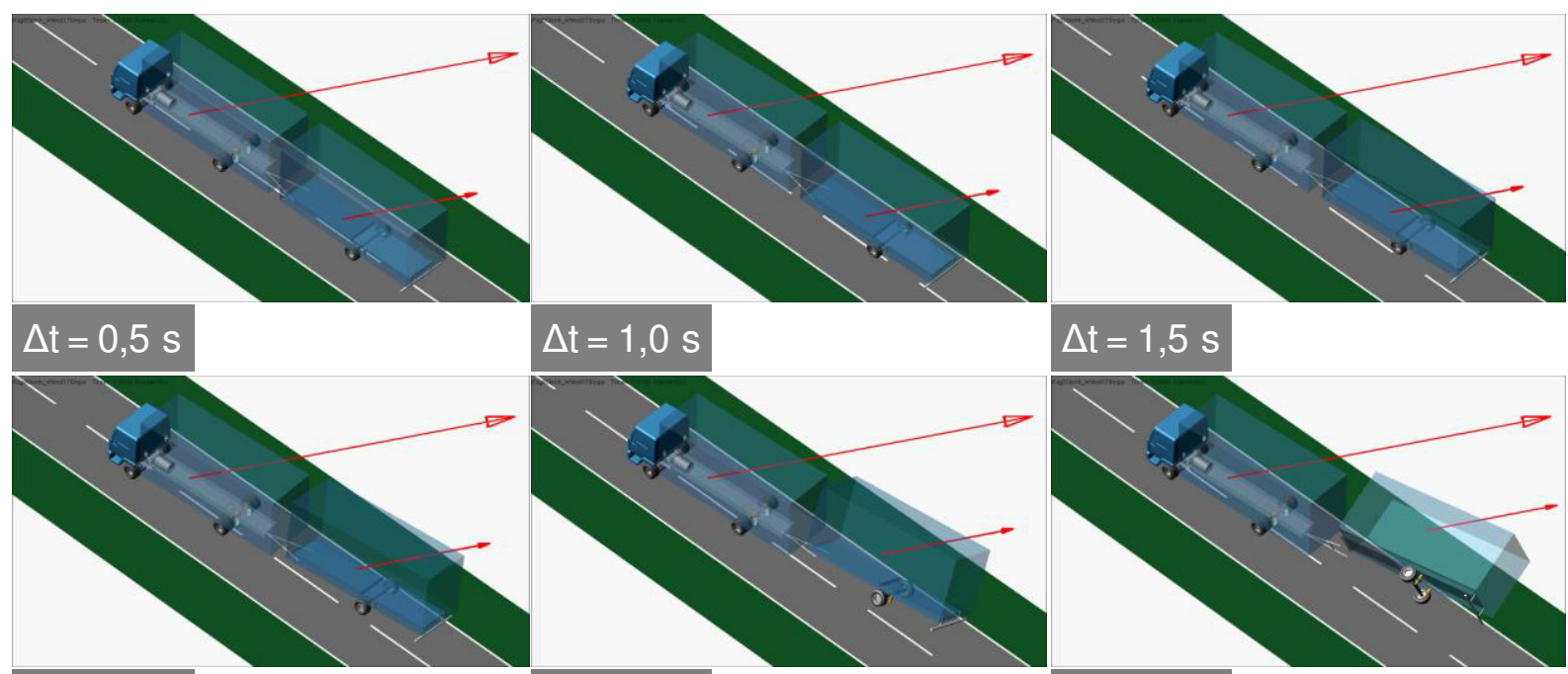

$\Delta \mathrm{t}=1,5 \mathrm{~s}$

$\Delta t=2,0 \mathrm{~s}$
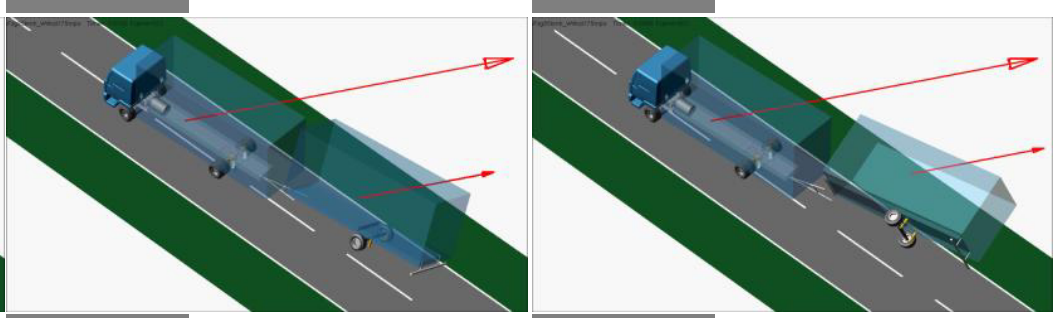

$\Delta t=2,5 \mathrm{~s}$

$\Delta t=3,0 s$

Abb. 5-49: Unbeladener $12 \mathrm{t}$-Zug unter Schräganströmung $\left(\mathrm{v}_{\mathrm{Fzg}}=85 \mathrm{~km} / \mathrm{h}\right.$, $\mathrm{a}_{\text {Wind }}=60^{\circ}\left(30^{\circ}\right.$ zur Querrichtung), $\left.v_{\text {Wind_direkt }}=17,5 \mathrm{~m} / \mathrm{s}(63 \mathrm{~km} / \mathrm{h})\right)$ - Kippen des Anhängers

Bezüglich der Windanfälligkeit unter Schräganströmung bleibt festzuhalten, dass diese sich ohne die Berücksichtigung eventueller aerodynamischer Einflüsse, welche auf Basis der vorliegenden Simulationsrandbedingungen nicht abgebildet werden können, weniger kritisch auf die Fahrdynamik auswirkt als die reine Queranströmung.

\subsubsection{Anhänger mit geöffneter Plane}

Eine in der Praxis vereinzelt anzutreffende Maßnahme zur Reduzierung der Windanfälligkeit des leeren Anhängers ist das Öffnen der seitlichen Plane, wodurch dem Wind kaum noch Angriffsfläche geboten wird. In der Simulation wird dieses Szenario dadurch abgebildet, dass die am Anhänger angreifende Windkraft zu Null gesetzt wird. Die Windkraft am Zugfahrzeug bleibt bestehen. Unter diesen Randbedingungen kommt es erst ab einer Windgeschwindigkeit von 22 m/s (79 km/h) zum Kippen. Dabei verlieren die windzugewandten Räder des Zugfahrzeugs unmittelbar nach Einsetzen des Windes den Kontakt zum Boden, wodurch der Anhänger zeitversetzt mitgerissen wird. Letzteres wird in der Bildfolge in Abb. 5-50 allerdings nicht mehr wiedergegeben, da die zugehörige Simulation vorher bereits abbricht.

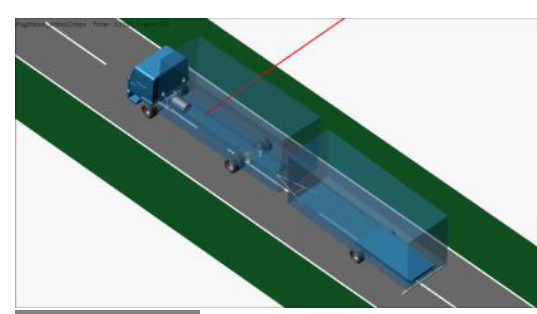

$\Delta t=0,2 s$

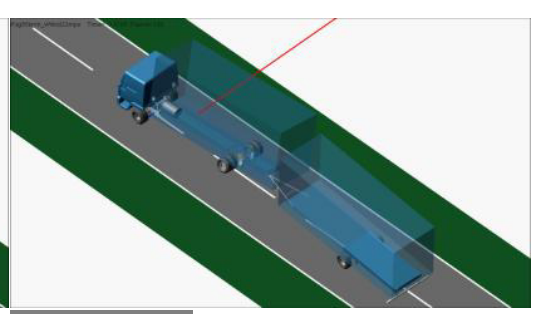

$\Delta \mathrm{t}=0,5 \mathrm{~s}$

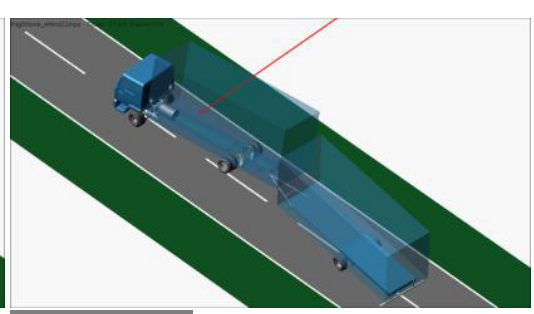

$\Delta \mathrm{t}=0,7 \mathrm{~s}$

Abb. 5-50: Unbeladener $12 \mathrm{t}-$ Zug, Windkraft nur auf Zugfahrzeug $\left(\mathrm{v}_{\mathrm{Fzg}}=85 \mathrm{~km} / \mathrm{h}\right.$, $\mathrm{v}_{\text {Wind_direkt }}=$ $22 \mathrm{~m} / \mathrm{s}(79 \mathrm{~km} / \mathrm{h}))$ - Kippen der Zugmaschine 
Auch wenn beim Öffnen der Plane Aspekte wie das Überschreiten der zulässigen Fahrzeugbreite aufgrund der aufgeschobenen Plane oder die Reduzierung der Aufbausteifigkeit zu beachten sind, scheint dies mit Hinblick auf die große Seitenwindanfälligkeit des leeren Anhängers eine Maßnahme zu sein, die gegebenenfalls in Betracht zu ziehen wäre.

\subsubsection{Einsatz von Ballasttanks bei Leerfahrten}

Eine im Rahmen des zum Projektabschluss durchgeführten zweiten Workshops diskutierte Maßnahme zur Reduzierung der Kippgefahr von leeren 12 t-Gliederzügen sieht die Verwendung von Ballasttanks vor. Diese sollten im Unterflurbereich von Zugfahrzeug und Anhänger befestigt werden und beispielweise mit Wasser (inklusive Frostschutz im Winter) oder Sand befüllt werden. Das Zusatzgewicht würde idealerweise der maximalen Zuladung des Anhängers (ca. 2,2 t) entsprechen. Somit könnte eine deutliche Absenkung des Schwerpunkts erreicht werden. Die Montage bzw. Demontage der Ballasttanks sollte mithilfe von Gabelstaplern möglich sein. Auch wenn diese Maßnahme unter ökologischen Gesichtspunkten (z.B. $\mathrm{CO}_{2}$-Bilanz) als kritisch angesehen werden kann und auch eine Umsetzbarkeit in der Praxis (Lagerung, Montage, Demontage, etc.) schwierig wäre, wird die Wirksamkeit im Rahmen entsprechender Simulationen untersucht. Dazu werden die Schwerpunktlagen von Zugfahrzeug und Anhänger des vollbeladenen Gespanns entsprechend abgesenkt. Infolgedessen fällt die Radlastverschiebung geringer aus und der Anhänger kippt erst bei einer Windgeschwindigkeit von $22 \mathrm{~m} / \mathrm{s}(79 \mathrm{~km} / \mathrm{h})$, wie in Abb. 5-51 dargestellt, und damit auch etwas später als beim regulär beladenen Gespann (vgl. Abb. 5-29).

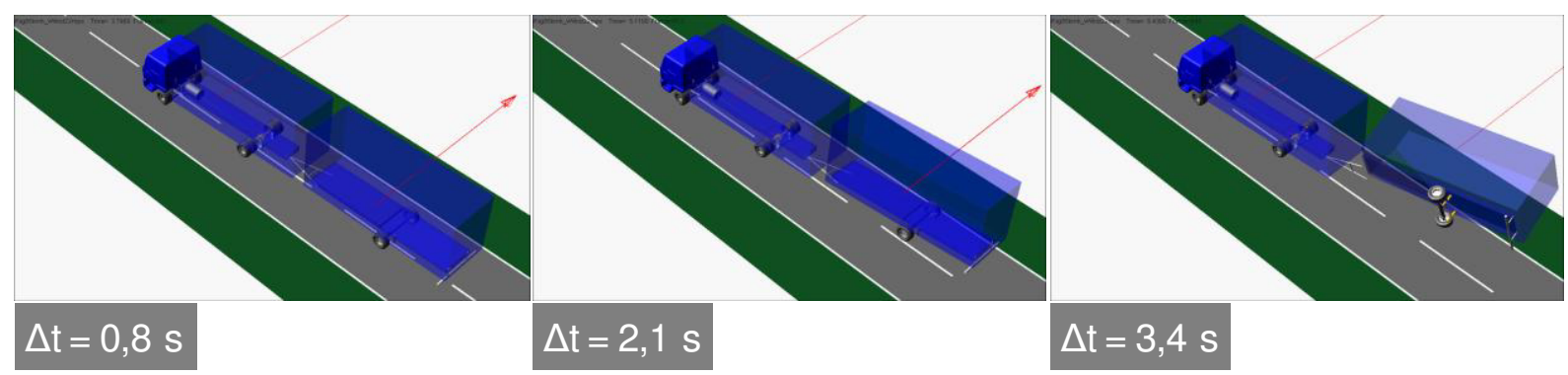

Abb. 5-51: $12 \mathrm{t}$-Zug mit Ballasttanks - entspricht beladenem Zustand mit abgesenktem Schwerpunkt $\left(\mathrm{V}_{\mathrm{Fzg}}=85 \mathrm{~km} / \mathrm{h}, \mathrm{v}_{\text {Wind_direkt }}=22 \mathrm{~m} / \mathrm{s}(79 \mathrm{~km} / \mathrm{h})\right)$

5.6

Fazit

Die Simulationsergebnisse prognostizieren, dass es für unbeladene 12 t-Leicht-Lkw-Kombinationen bereits ab einer Windgeschwindigkeit von $16 \mathrm{~m} / \mathrm{s}(57,6 \mathrm{~km} / \mathrm{h})$, d.h. bei Windstärke 7, auch bei einem sehr gut reagierenden Fahrer zu einem Kippen des Anhängers kommen kann. Zuvor treten bereits hohe Spurabweichungen auf. Diese kritische Windgeschwindigkeit kann sich für einen schlecht reagierenden Fahrer nochmals um bis zu $2 \mathrm{~m} / \mathrm{s}$ nach unten verschieben, wie die Ergebnisse in Kapitel 5.5.4.2 zeigen.

Im vollbeladenen Zustand kann ab einer Windgeschwindigkeit von $21,5 \mathrm{~m} / \mathrm{s}(77,4 \mathrm{~km} / \mathrm{h})$, was Windstärke 9 entspricht, ein Kippen des Anhängers einsetzen. Dabei wird deutlich, dass 
durch Maßnahmen, die eine Erhöhung des Anhängergewichts bei Leerfahrten vorsehen, wie beispielsweise die in Kapitel 5.5.4.10 betrachteten Ballasttanks, die Kippgefahr grundsätzlich nur im begrenzten Maße gesenkt werden kann. Bei Sturm käme es hier ebenfalls zu einem Kippen des Anhängers.

Die Implementierung von Sturmstützen, als weitere mögliche Maßnahme, birgt fahrdynamische Risiken, da das Zugfahrzeug hier regelrecht ausgehebelt wird. Zudem zeigen die entsprechenden Simulationen in Kapitel 5.5.3, dass diese nur bei sehr kurzen Böen wirksam sind und ansonsten kaum für eine Minderung der Kippgefahr sorgen.

Die Simulationsergebnisse sind im Rahmen eines zweiten Workshops zum Abschluss des Projekts vorgestellt und mit den Teilnehmern diskutiert worden. Hierzu zählen u.a. Vertreter des Bundesverbands Güterkraftverkehr Logistik und Entsorgung (BGL), der Deutschen Gesetzlichen Unfallversicherung (DGUV) sowie einer Spedition. Neben den bereits angeführten Maßnahmen zur Reduzierung der Kippgefahr wird hier auch ein grundsätzliches Fahrverbot von Leicht-Lkw-Kombinationen bei Sturmereignissen angeführt. Basieren könnte dies auf offiziellen Warnungen der Wetterdienste, welche in der Regel mit einer Vorlaufzeit von ca. 24 Stunden ausgesprochen werden können. Denkbar wären entsprechende Radiodurchsagen oder die Nutzung der in heutigen Fahrzeugen verbreitet installierten Fleetboard-Systeme. Dabei sollten nach Ansicht von Workshop-Teilnehmern die Unternehmen direkt in die Verantwortung genommen werden, d.h. die Entscheidung sollte nicht den Fahrern überlassen werden, da diese dadurch unter zusätzlichen Druck geraten würden. Die Unternehmer müssten im Rahmen einer Gefährdungsanalyse die Verkehrssicherheit ihrer Fahrzeuge sicherstellen und bei entsprechenden Wetterlagen die Weiterfahrt untersagen. Auch wird in der Definition eines Mindestleergewichts in Abhängigkeit der Seitenfläche im Rahmen der Zulassungsvorschriften eine weitere Möglichkeit zur Adressierung der Seitenwindproblematik bei leichten Gliederzügen gesehen. 


\section{Zusammenfassung}

Im Rahmen dieses Projektes wurde das Unfallgeschehen von Lkw mit einem zulässigen Gesamtgewicht über 3,5 t in Deutschland näher beleuchtet. Ein besonderer Fokus lag dabei auf Gliederzügen mit extrem leichter Bauweise, welche in den letzten Jahren zunehmend Verbreitung gefunden haben und die bis Oktober 2015 eine Umgehung der 2005 für Lkw ab 12 t eingeführten Mautpflicht auf deutschen Fernstraßen ermöglichten. Problematisch ist in erster Linie die hohe Windanfälligkeit dieser Leicht-Lkw, welche vollbeladen und bei gleichen Abmessungen immer noch ca. $3 \mathrm{t}$ weniger wiegen als übliche Fernlastzüge im leeren Zustand. Mittels einer Simulationsstudie wurde die Kippstabilität dieser Fahrzeuge unter Seitenwind, auch im Vergleich zu 40 t-Gliederzügen, umfassend untersucht.

Ausgangspunkt der Untersuchungen bildeten eine Charakterisierung der verschiedenen Fahrzeugtypen bzw. Gespanne, eine Betrachtung der Normen und Anforderungen im Bereich der Lkw-Sicherheit sowie eine Analyse des Verkehrsaufkommens von Lkw in Deutschland. Dabei ist das Verkehrsaufkommen von Leicht-Lkw-Gespannen auf Basis von Verkehrszählungen an verschiedenen Autobahnabschnitten näher erfasst worden. Der ermittelte Anteil von 12 t-Leicht-Lkw-Kombinationen an der Gesamtzahl der Fahrzeuge mit einem zulässigen Gesamtgewicht größer 3,5 t liegt zwischen 1,5\% und 2,6\%, was durch Erhebungen der BASt bestätigt wird. Des Weiteren sind neben der Analyse des Verkehrsaufkommens auch Erkenntnisse aus der Kontrollstatistik in Hinblick auf Sozialvorschriften, Ladungssicherung sowie technischer Rahmenbedingungen betrachtet worden. Probleme infolge der Arbeitsbedingungen von Lkw-Fahrern wurden in diesem Zusammenhang ebenfalls thematisiert.

Einen zentralen Bestandteil des Projekts bildete die Analyse des Unfallgeschehens von Lkw mit einem zulässigen Gesamtgewicht größer 3,5 t in Deutschland. Die Ergebnisse basieren sowohl auf Daten des Statistischen Bundesamtes (Destatis) als auch auf Auswertungen der Unfalldatenbank der Versicherer (UDB). Untersuchte Merkmale sind u.a. Ortslagen, Art der beteiligten Lkw und Unfallgegner sowie die jeweiligen Unfallarten, -typen und -folgen. Ferner wurden auch Fehlverhalten und der Anteil Hauptverursacher für die beteiligten Lkw-Fahrer betrachtet. Ein weiterer Fokus der Unfallanalyse lag wiederum auf 12 t-Leicht-Lkw-Kombinationen. Um deren Unfallgeschehen näher charakterisieren zu können, wurden im Zuge einer Sonderabfrage beim Statistischen Bundesamt entsprechende fahrzeugspezifische Auswertungen der Bundesstatistik analysiert. Insgesamt fallen die im Rahmen der Sonderauswertung erzielten Fallzahlen für Leicht-Lkw-Unfälle unter Seitenwind, gerade mit Hinblick auf die untersuchte Zeitspanne, äußerst gering aus. Dennoch machen in der polizeilichen Unfallaufnahme "Seitenwind“ und "Unwetter" zusammen genommen bei Leicht-Lkw-Kombinationen einen Anteil von 17\% der genannten äußeren Unfallursachen aus, für die übrigen Gliederzüge betrug dieser Anteil nur rund 4\%. Die Auswertungen legen den Schluss nahe, dass die amtliche Statistik aus verschiedenen Gründen die absolute Zahl der Unfallereignisse von 12 t-Leicht-Lkw-Kombinationen infolge von Seitenwind unterschätzt.

Teil der durchgeführten Unfallanalyse war auch der Aufbau und die Auswertung einer Unfalldatenbank mit 150 recherchierten und auf Seitenwind zurückzuführenden Leicht-Lkw-Un- 
fällen. Die Auswertung der Unfalldatenbank zeigt, dass im Grunde alle in der Datenbank enthaltenen Fälle in Zusammenhang mit Sturmtiefs gebracht werden können. Die Monate mit hohem Sturmaufkommen entsprechen den Monaten mit erhöhtem Unfallaufkommen in der Datenbank. Zu nennen sind in diesem Zusammenhang erwartungsgemäß die Herbst- und Wintermonate, vor allem der Zeitraum Dezember bis März. Die hier für die Sturmereignisse in den jeweiligen Monaten ermittelten Durchschnittswerte der Windgeschwindigkeit liegen zwischen 95 und $101 \mathrm{~km} / \mathrm{h}$. Die dokumentierten Unfälle finden fast ausschließlich außerorts statt, oftmals auf Straßen ohne seitlichen Bewuchs. Dabei liegt ein erheblicher Anteil der Unfallstellen an Brücken. Ein charakteristisches Merkmal ist zudem der hohe Anteil von Unfällen ohne Personenschaden, was einer der Gründe für die eventuelle Unterschätzung dieser Unfallereignisse anhand der amtlichen Statistik sein könnte. Kennzeichnend für Leicht-Lkw-Unfälle unter Seitenwind ist auch die Tatsache, dass die Fahrzeuge oft im unbeladenen Zustand verunglücken. Das gilt insbesondere für den Anhänger. In zwei Dritteln der Fälle mit bekanntem Beladungszustand war das gesamte Gespann oder mindestens der Anhänger bzw. das Zugfahrzeug leer unterwegs.

In der Simulationsstudie zur Kippstabilität von 40 t- und 12 t-Gliederzügen unter Seitenwind konnte gezeigt werden, dass es für unbeladene $12 \mathrm{t}$-Leicht-Lkw-Kombinationen schon ab einer mittleren Windgeschwindigkeit von $16 \mathrm{~m} / \mathrm{s}(57,6 \mathrm{~km} / \mathrm{h})$, d.h. bei Windstärke 7, auch bei einem sehr gut reagierenden Fahrer zu einem Kippen des Anhängers kommen kann. Zuvor treten bereits hohe Spurabweichungen auf. Im vollbeladenen Zustand kann ab einer mittleren Windgeschwindigkeit von $21,5 \mathrm{~m} / \mathrm{s}(77,4 \mathrm{~km} / \mathrm{h})$, was Windstärke 9 entspricht, ein Kippen des Anhängers einsetzen. Hinsichtlich der Simulationsergebnisse ist zu beachten, dass der Luftwiderstandsbeiwert in Querrichtung die einzige aerodynamische Einflussgröße im Rahmen des definierten Seitenwindmodells darstellt. Mögliche weitere aerodynamische Einflüsse, beispielsweise aufgrund der Längsbewegung des Fahrzeugs, können in der Mehrkörpersimulationsumgebung nicht abgebildet werden. Somit ist die Höhe des Luftwiderstandsbeiwerts maßgeblich für die in der Simulation auftretende Windlast. Da die im Rahmen des Projektes ausgewerteten Literaturquellen eine breite Spanne möglicher Werte offenbaren, sollten die in der Simulation ermittelten kritischen mittleren Windgeschwindigkeiten als Richtwerte aufgefasst werden, ab welcher generell ein Kippen des Anhängers zu erwarten ist. Dabei kann nicht ausgeschlossen werden, dass dies in Abhängigkeit der Randbedingungen in der Realität auch bei geringerer oder höherer Windgeschwindigkeit erfolgen kann.

Anhand der durchgeführten Simulationen wird deutlich, dass durch Maßnahmen, die eine Erhöhung des Anhängergewichts bei Leerfahrten vorsehen, wie beispielsweise die untersuchten zusätzlichen Ballasttanks, die Kippgefahr grundsätzlich nur im begrenzten Maße reduziert werden kann. Bei Sturmböen käme es hier ebenfalls zu einem Kippen des Anhängers. Die Implementierung von Sturmstützen, als weitere mögliche Maßnahme, birgt fahrdynamische Risiken, da die Simulation erwarten lässt, dass das Zugfahrzeug hier regelrecht ausgehebelt wird. Zudem zeigen die entsprechenden Simulationen, dass die Sturmstützen nur bei sehr kurzen Böen wirksam sind und ansonsten kaum für eine Minderung der Kippgefahr sorgen. 
Im Rahmen eines Workshops zum Abschluss des Projekts wurde hinsichtlich der Reduzierung der Kippgefahr auch ein grundsätzliches Fahrverbot von Leicht-Lkw-Kombinationen bei Sturmereignissen diskutiert. Basieren könnte dies auf offiziellen Warnungen der Wetterdienste. Denkbar wären entsprechende Radiodurchsagen oder die Nutzung der in heutigen Fahrzeugen verbreitet installierten Fleetboard-Systeme. Dabei wird es als sinnvoll erachtet, die Unternehmen direkt in die Verantwortung zu nehmen, d.h. die Entscheidung zur Weiterfahrt sollte nicht den Fahrern überlassen werden, da diese dadurch unter zusätzlichen Druck geraten würden. Die Unternehmer sollten im Rahmen einer Gefährdungsanalyse die Verkehrssicherheit ihrer Fahrzeuge sicherstellen und bei entsprechenden Wetterlagen die Weiterfahrt untersagen. Auch wäre die Definition eines Mindestleergewichts in Abhängigkeit der Seitenfläche im Rahmen der Zulassungsvorschriften eine weitere Möglichkeit zur Adressierung der Seitenwindproblematik bei leichten Gliederzügen. 


\section{Abkürzungen}

AEB Autonomous Emergency Braking

AIS Abbreviated Injury Scale

BAG Bundesamt für Güterverkehr

BASt Bundesanstalt für Straßenwesen

BGL Bundesverband Güterkraftverkehr Logistik und Entsorgung

Destatis Statistisches Bundesamt

DGUV Deutsche Gesetzliche Unfallversicherung

DIN Deutsche Institut für Normung

ECE Economic Commission for Europe

ESC Electronic Stability Control

ESP Elektronisches Stabilitätsprogramm

EU-15 Belgien, Deutschland, Frankreich, Italien, Luxemburg, Niederlande, Dänemark, Irland, Vereinigtes Königreich, Griechenland, Portugal, Spanien, Finnland, Österreich, Schweden

IBC Intermediate Bulk Container

KBA Kraftfahrt-Bundesamt

LDW Lane Departure Warning

MAIS Maximum AIS

StVZO Straßenverkehrs-Zulassungs-Ordnung

TRL Transport Research Laboratory

UDB Unfalldatenbank der Versicherer

UDV Unfallforschung der Versicherer

VDI Verein Deutscher Ingenieure

zGG zulässiges Gesamtgewicht 
8 Literatur

[ADA12] N.N.

ADAC Online

ADAC Test LKW Unterfahrschutz - Standard-Schutz kann tödlich enden https://www.adac.de/infotestrat/tests/crash-test/unterfahrschutz_lkw_2012 München, 2012

[ADA14] ADAMSKI, D.

Simulation in der Fahrwerktechnik

Springer Vieweg, Wiesbaden, 2014

[ALA07] ALAM, F.; WATKINS, S.

Effects of Crosswinds on Double Stacked Container Wagons

$16^{\text {th }}$ Australiasian Mechanics Conference, Australia, 2007

[BAG14] N.N.

Bundesamt für Güterverkehr (BAG)

Hinweise zu den Sozialvorschriften im Straßenverkehr

https://www.bag.bund.de/DE/Navigation/Rechtsvorschriften/Lenk-Ruhezeiten/

lenk-ruhezeiten_node.html

Leitfaden_Rechtsvorschriften_2014.pdf

September 2014

[BAG16] N.N.

Verkehrsaufgaben > Straßenkontrollen

Bundesamt für Güterverkehr (BAG)

https://www.bag.bund.de/DE/Navigation/Verkehrsaufgaben/Kontrollen/Strassenk

ontrollen/strassenkontrollen_node.html

Köln, 2016

[BAS09] N.N.

Bundesanstalt für Straßenwesen (BASt)

Auswirkungen von Belastungen und Stress auf das Verkehrsverhalten von Lkw-

Fahrern

Anhang D - Ergebnispräsentation Experten-Workshop

Bergisch Gladbach, 2009

[BLY08] BLYTHE, W.

Application of HVE SIMON to the Analysis of Lateral Wind Loadings on Highsided Vehicles

Palo Alto, California, 2008 
[BRÜ14] BRÜNGLINGHAUS, C.

"Non-Hit Car and Truck"-Projekt: Volvo arbeitet an Rundumsicht von Pkw und Lkw www.springerprofessional.de / Automobil + Motoren / Onlineartikel

21.Oktober 2014

[CHE11] CHELI, F.; RIPAMONTI, F.; SABBIONI, E.; TOMASINI, G.

Wind tunnel tests on heavy road vehicles: Cross wind induced loads - Part 2 Journal of Wind Engineering and Industrial Aerodynamics 2011

[DIN01] N.N.

DIN EN 12640

Ladungssicherung auf Straßenfahrzeugen - Zurrpunkte an Nutzfahrzeugen zur Güterbeförderung - Mindestanforderungen und Prüfung Januar 2001

[DIN07] N.N.

DIN EN 12642

Ladungssicherung auf Straßenfahrzeugen - Aufbauten an Nutzfahrzeugen Mindestanforderungen

Deutsche Fassung EN 12642:2006

Januar 2007

[DIN11] N.N.

DIN EN 12195-1

Ladungssicherung auf Straßenfahrzeugen - Sicherheit - Teil 1: Berechnung von Sicherungskräften

Deutsche Fassung EN 12195-1:2010 - Nationales Vorwort Juni 2011

[DWD16] N.N.

Windwarnskala

Deutscher Wetterdienst (DWD)

http://www.wettergefahren.de/warnungen/windwarnskala.html

Offenbach, 2016

[ECE08] N.N.

Regelung Nr. 58 der Wirtschaftskommission der Vereinten Nationen für Europa (UN/ECE)

Einheitliche Bedingungen für die Genehmigung von: I. Einrichtungen für den hinteren Unterfahrschutz / II. Fahrzeugen hinsichtlich des Anbaus von Einrichtungen eines genehmigten Typs für den hinteren Unterfahrschutz / III. Fahrzeugen hinsichtlich ihres hinteren Unterfahrschutzes - Revision 2 Amtsblatt der Europäischen Union, L 232/13

30. August 2008 
[ECE10] N.N.

Regelung Nr. 29 der UN-Wirtschaftskommission für Europa (UN/ECE)

Einheitliche Bedingungen für die Genehmigung der Fahrzeuge hinsichtlich des

Schutzes von Insassen des Fahrerhauses von Nutzfahrzeugen

Änderungsserie 03

Amtsblatt der Europäischen Union, L 304/21

20. November 2010

[ECE10a] N.N.

Regelung Nr. 93 der Wirtschaftskommission der Vereinten Nationen für Europa (UN/ECE)

Einheitliche Bedingungen für die Genehmigung von: I. Einrichtungen für den vorderen Unterfahrschutz / II. Fahrzeugen hinsichtlich des Anbaus einer Einrichtung eines genehmigten Typs für den vorderen Unterfahrschutz / III. Fahrzeugen hinsichtlich ihres vorderen Unterfahrschutzes

Amtsblatt der Europäischen Union, L 185/56

17. Juli 2010

[ECE12] N.N.

Regelung Nr. 73 der Wirtschaftskommission der Vereinten Nationen für Europa (UN/ECE)

Einheitliche Bedingungen für die Genehmigung von: I. Fahrzeugen hinsichtlich ihrer seitlichen Schutzeinrichtungen / II. seitlichen Schutzeinrichtungen / III. Fahrzeugen hinsichtlich des Anbaus einer nach Teil II dieser Regelung typgenehmigten seitlichen Schutzeinrichtung

Amtsblatt der Europäischen Union, L 122/1

8. Mai 2012

[ECE15] N.N.

Proposal for the 03 series of amendments to Regulation No. 58 (Rear underrun protection) - Submitted by the Working Party on General Safety Provisions ECE/TRANS/WP.29/2015/85

United Nations Economic Commission for Europe - Inland Transport Committee World Forum for Harmonization of Vehicle Regulations

167th session

Genf, 10.-13. November 2015

[EGV09] N.N.

Verordnung (EG) Nr. 661/2009 des Europäischen Parlaments und des Rates vom 13. Juli 2009 über die Typgenehmigung von Kraftfahrzeugen, Kraftfahrzeuganhängern und von Systemen, Bauteilen und selbstständigen technischen Einheiten für diese Fahrzeuge hinsichtlich ihrer allgemeinen Sicherheit Amtsblatt der Europäischen Union, L 200/1

31. Juli 2009 
[EGV12] N.N.

Verordnung (EU) Nr. 347/2012 der Kommission vom 16. April 2012 zur Durchführung der Verordnung (EG) Nr. 661/2009 des Europäischen Parlaments und des Rates über die Typgenehmigung von Notbremsassistenzsystemen für bestimmte Kraftfahrzeugklassen

Amtsblatt der Europäischen Union, L 109/1

21. April 2012

[EVE10] EVERS, C.

Bundesanstalt für Straßenwesen (BASt)

Forschung kompakt; Ausgabe 06/10

Auswirkungen von Belastungen und Stress auf das Verkehrsverhalten von Lkw-

Fahrern

Bergisch Gladbach, 2010

[EVE11] EVERS, C.

Verkehrsunfall und Fahrzeugtechnik (VKU)

Auswirkungen von Belastungen und Stress auf das Verkehrsverhalten von LkwFahrern

Fachmagazin, Ausgabe Januar 2011

Springer Fachmedien, München, 2011

[FRA13] FRASQUET, C.; INDINGER, T.

Numerische Untersuchungen zur Aerodynamik von Nutzfahrzeugkombinationen bei realitätsnahen Fahrbedingungen unter Seitenwindeinfluss

Forschungsvereinigung Automobiltechnik e.V. (FAT)

FAT-Schriftenreihe 260

Berlin, 2013

[HOE13] HOEPKE, E.; BREUER, S.

Nutzfahrzeugtechnik - Grundlagen, Systeme, Komponenten

7., überarbeitete und erweiterte Auflage

ATZ/MTZ-Fachbuch

Springer Vieweg, Wiesbaden, 2013

[HUC11] HUCHO, W.

Aerodynamik der stumpfen Körper

Vieweg+Teubner Verlag, Wiesbaden, 2011

[KBA11] N.N.

Verkehr deutscher Lastkraftfahrzeuge (VD) - Verkehrsaufkommen - Jahr 2010

VD 1

Kraftfahrt-Bundesamt - Statistik

Flensburg, Juli 2011 
[KBA12] N.N.

Verkehr deutscher Lastkraftfahrzeuge (VD) - Verkehrsaufkommen - Jahr 2011 VD 1

Kraftfahrt-Bundesamt - Statistik

Flensburg, August 2012

[KBA13] N.N.

Verkehr deutscher Lastkraftfahrzeuge (VD) - Verkehrsaufkommen - Jahr 2012 VD 1

Kraftfahrt-Bundesamt - Statistik

Flensburg, August 2013

[KBA14] N.N.

Verkehr deutscher Lastkraftfahrzeuge (VD) - Verkehrsaufkommen - Jahr 2013 VD 1

Kraftfahrt-Bundesamt - Statistik

Flensburg, Oktober 2014

[KBA15] N.N.

Verkehr deutscher Lastkraftfahrzeuge (VD) - Verkehrsaufkommen - Jahr 2014 VD 1

Kraftfahrt-Bundesamt - Statistik

Flensburg, November 2015

[KBA15a] N.N.

Fahrzeugzulassungen (FZ) - Bestand an Nutzfahrzeugen, Kraftfahrzeugen insgesamt und Kraftfahrzeuganhängern nach technischen Daten (Größenklassen, Motorisierung, Fahrzeugklassen und Aufbauarten) - 1. Januar 2015 FZ 25

Kraftfahrt-Bundesamt - Statistik

Flensburg, Dezember 2015

[KEM15] KEMMNITZ, W.

Implementierung von Sturmstützen an Leicht-Lkw-Anhängern - Bildmaterial ORTEN Fahrzeugbau GmbH

Bernkastel-Kues

2015

[KIM13] KIMMERLE, H.

Internationaler Erfahrungsaustausch an der Akademie der Polizei BW in Freiburg Erkennen von Manipulationen bei digitalen Tachographen Polizei-Verkehr-Technik (PVT) - Fachzeitschrift der Polizei Ausgabe 4/13, Seite 13 - 16 2013 
[KRA09] KRAMER, F.

Passive Sicherheit von Kraftfahrzeugen

Biomechanik - Simulation - Sicherheit im Entwicklungsprozess

3., überarbeitete Auflage

Vieweg+Teubner, GWV Fachverlage GmbH, Wíesbaden 2009

[LAU15] LAUENROTH, L.

„Wir haben die Nutzlast erhöht“ - Interview mit Simeon Breuer, Geschäftsführer

der LIT Speditions GmbH

DVZ - Deutsche Verkehrs-Zeitung - Rubrik Straße

www.dvz.de

3. Dezember 2015

[LOH14] LOHRE, D.; BEMECKER, T.; STOCK, W.

Institut für Nachhaltigkeit in Verkehr und Logistik, Hochschule Heilbronn

ZF Friedrichshafen AG

ZF Zukunftsstudie Fernfahrer 2.0 - Der Mensch im Transport- und Logistikmarkt Euro TransportMedia Verlags- und Veranstaltungs-GmbH

Stuttgart, September 2014

[PET13] PETERSEN, E.

Fahrerassistenzsysteme für Nutzfahrzeuge zur Erhöhung der Verkehrssicherheit

- Teil 2

VKU - Verkehrsunfall und Fahrzeugtechnik - Ausgabe 1/2013

2.2 Unfallforschung, Seite $22-32$

Springer Automotive Media

München, Januar 2013

[PRO15] PROPPE, C.

Gutachten zum Schaltprogramm der Windwarnanlage an der B207

Karlsruher Institut für Technologie (KIT)

Karlsruhe, 2015

[RAT14] RATHMANN, M.

Future Truck - Vernetzter Lkw fährt komplett autonom

www.eurotransport.de / Fahrzeuge

3. Juli 2014

[RAT15] RATHMANN, M.

Gebührenpflicht ab 7,5 Tonnen - Keine Angst vor der Maut www.eurotransport.de / Transportwelt / Spedition und Logistik

9. Juli 2015 
[STA11] N.N.

Verkehrsunfälle - Unfälle von Güterkraftfahrzeugen im Straßenverkehr - 2010 Statistisches Bundesamt

Artikelnummer: 5462410-10700-4 [PDF]

www.destatis.de

Wiesbaden, 30. November 2011

[STA12] N.N.

Verkehrsunfälle - Unfälle von Güterkraftfahrzeugen im Straßenverkehr - 2011 Statistisches Bundesamt

Artikelnummer: 5462410-11700-4 [PDF]

www.destatis.de

Wiesbaden, 14. November 2012

[STA13] N.N.

Verkehrsunfälle - Unfälle von Güterkraftfahrzeugen im Straßenverkehr - 2012

Statistisches Bundesamt

Artikelnummer: 5462410-12700-4 [PDF]

www.destatis.de

Wiesbaden, 26. November 2013

[STA14] N.N.

Verkehrsunfälle - Unfälle von Güterkraftfahrzeugen im Straßenverkehr - 2013

Statistisches Bundesamt

Artikelnummer: 5462410-13700-4 [PDF]

www.destatis.de

Wiesbaden, 18. November 2014

[STA14a] N.N.

Verkehr - Verkehrsunfälle 2013

Statistisches Bundesamt, Fachserie 8, Reihe 7

Artikelnummer: 2080700137004

www.destatis.de

Wiesbaden, 2. Juli 2014

[STA15] N.N.

Verkehrsunfälle - Unfälle von Güterkraftfahrzeugen im Straßenverkehr - 2014 Statistisches Bundesamt

Artikelnummer: 5462410-14700-4 [PDF]

www.destatis.de

Wiesbaden, 17. Dezember 2015 
[STA15a] N.N.

Seitens der fka in Auftrag gegebene Destatis Sonderauswertung Statistisches Bundesamt

Verkehrsunfallstatistik

Wiesbaden, 19. Februar 2015

[VDI04] N.N.

VDI-Richtlinie 2700 - Ladungssicherung auf Straßenfahrzeugen Verein Deutscher Ingenieure

November 2004 


\section{$9 \quad$ Anhang}

9.1 Bundesstatistik im Vergleich zur Unfalldatenbank der Versicherer

\subsubsection{Ortslage}

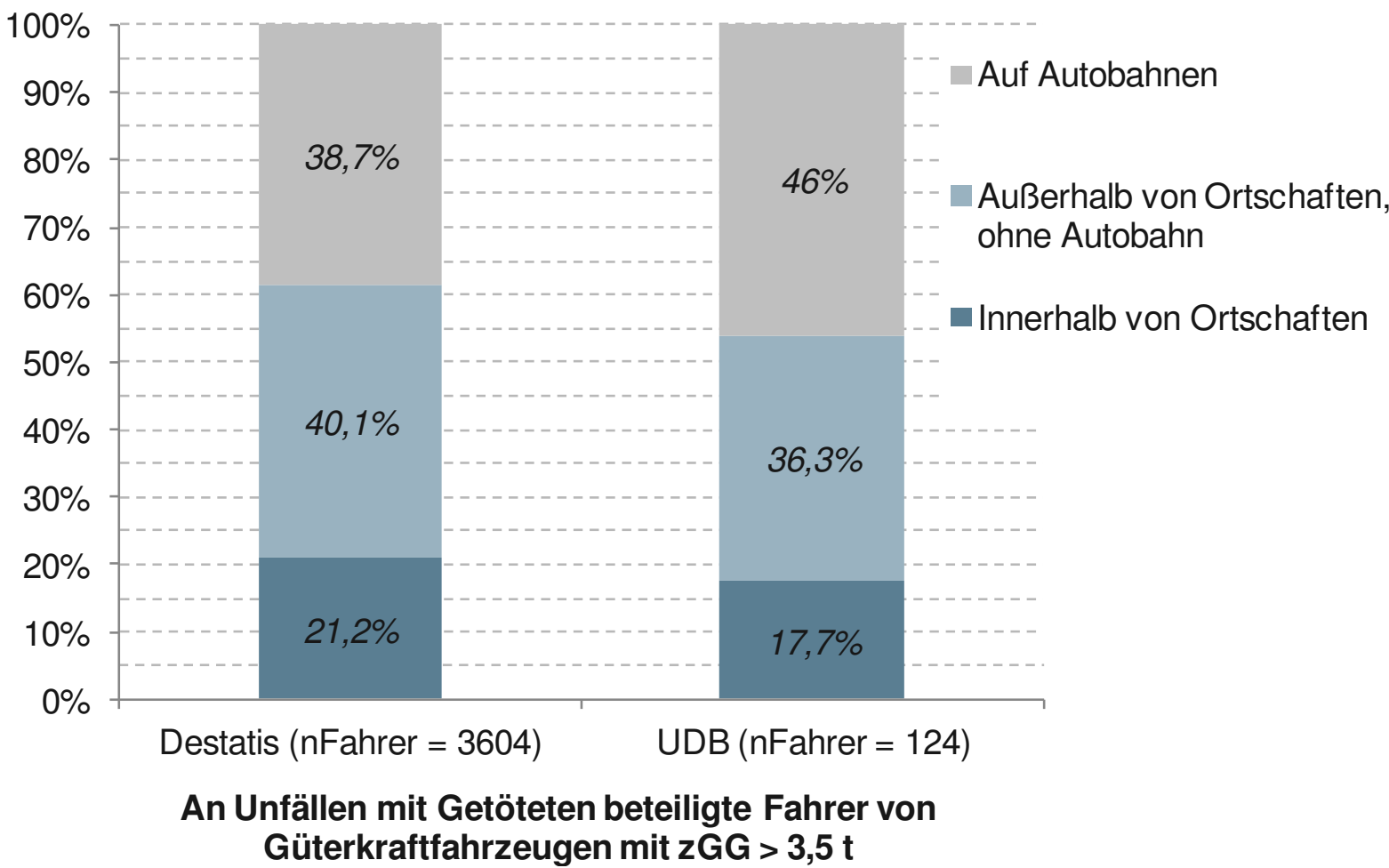

Abb. 9-1: Vergleich Destatis (2010-2014) [STA11-15] \& UDB nach Ortslage

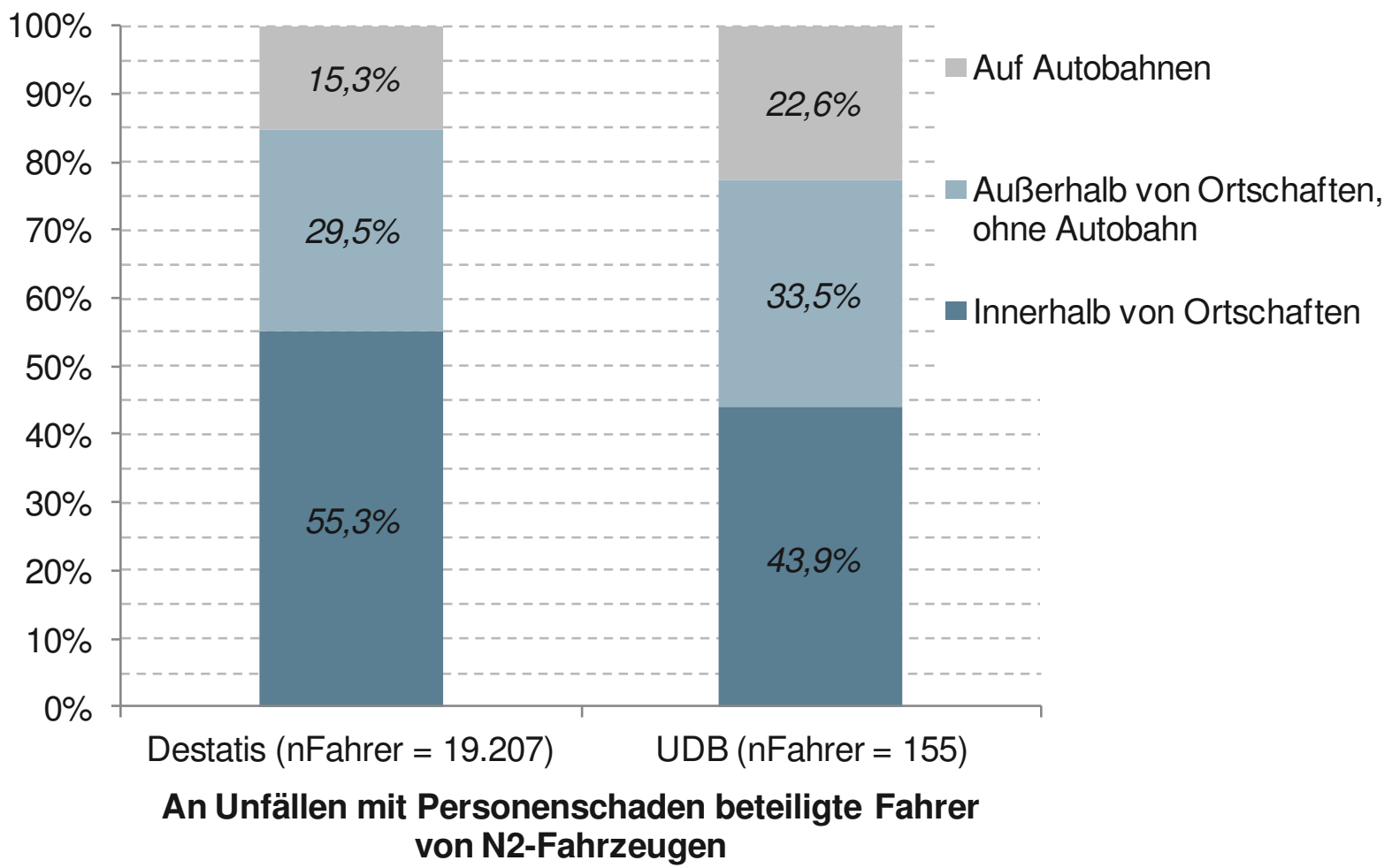

Abb. 9-2: Vergleich Destatis (2010-2014) [STA11-15] \& UDB nach Ortslage 


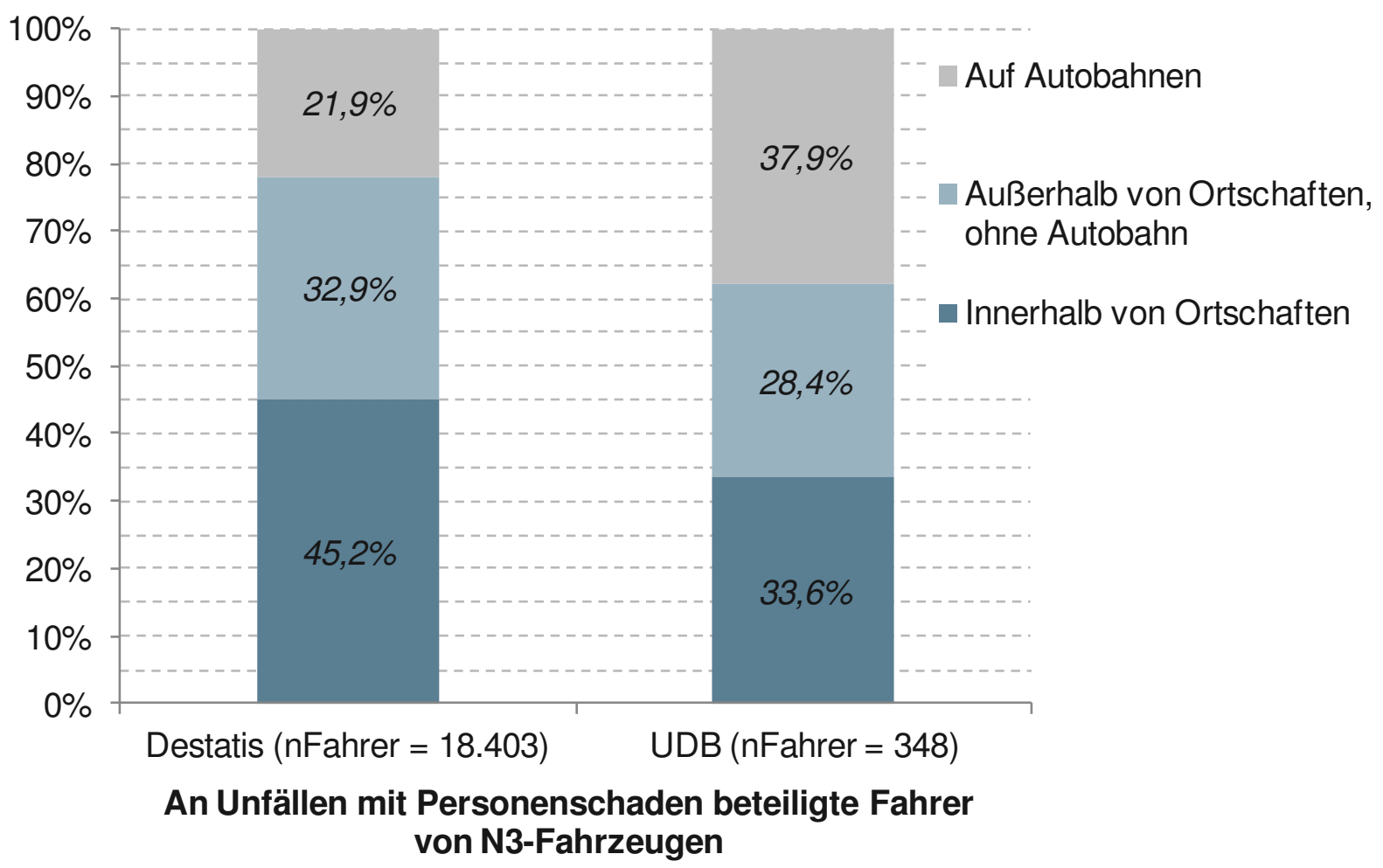

Abb. 9-3: Vergleich Destatis (2010-2014) [STA11-15] \& UDB nach Ortslage

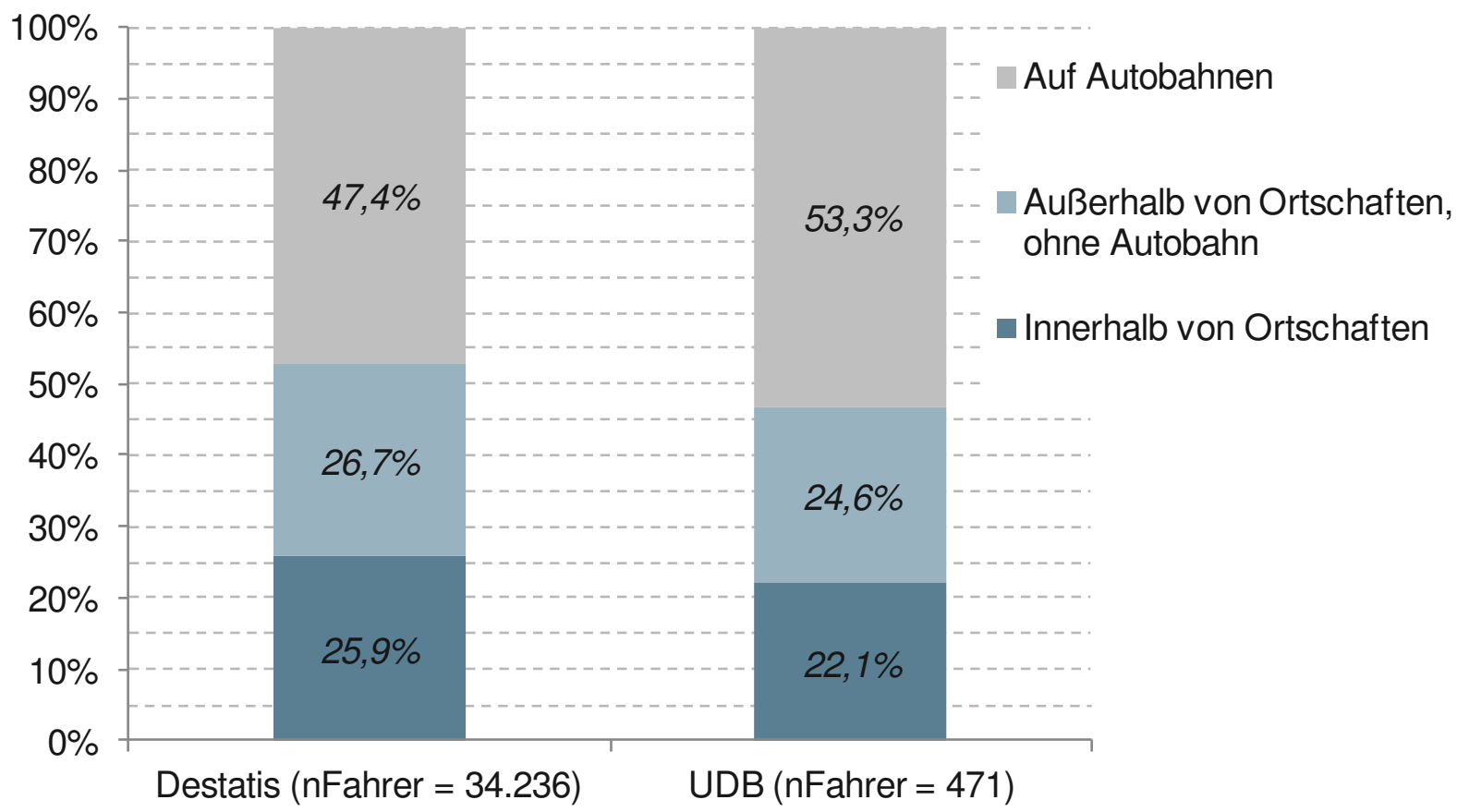

An Unfällen mit Personenschaden beteiligte Fahrer von

Sattelzugmaschinen

Abb. 9-4: Vergleich Destatis (2010-2014) [STA11-15] \& UDB nach Ortslage 


\subsubsection{Unfallfolgen}

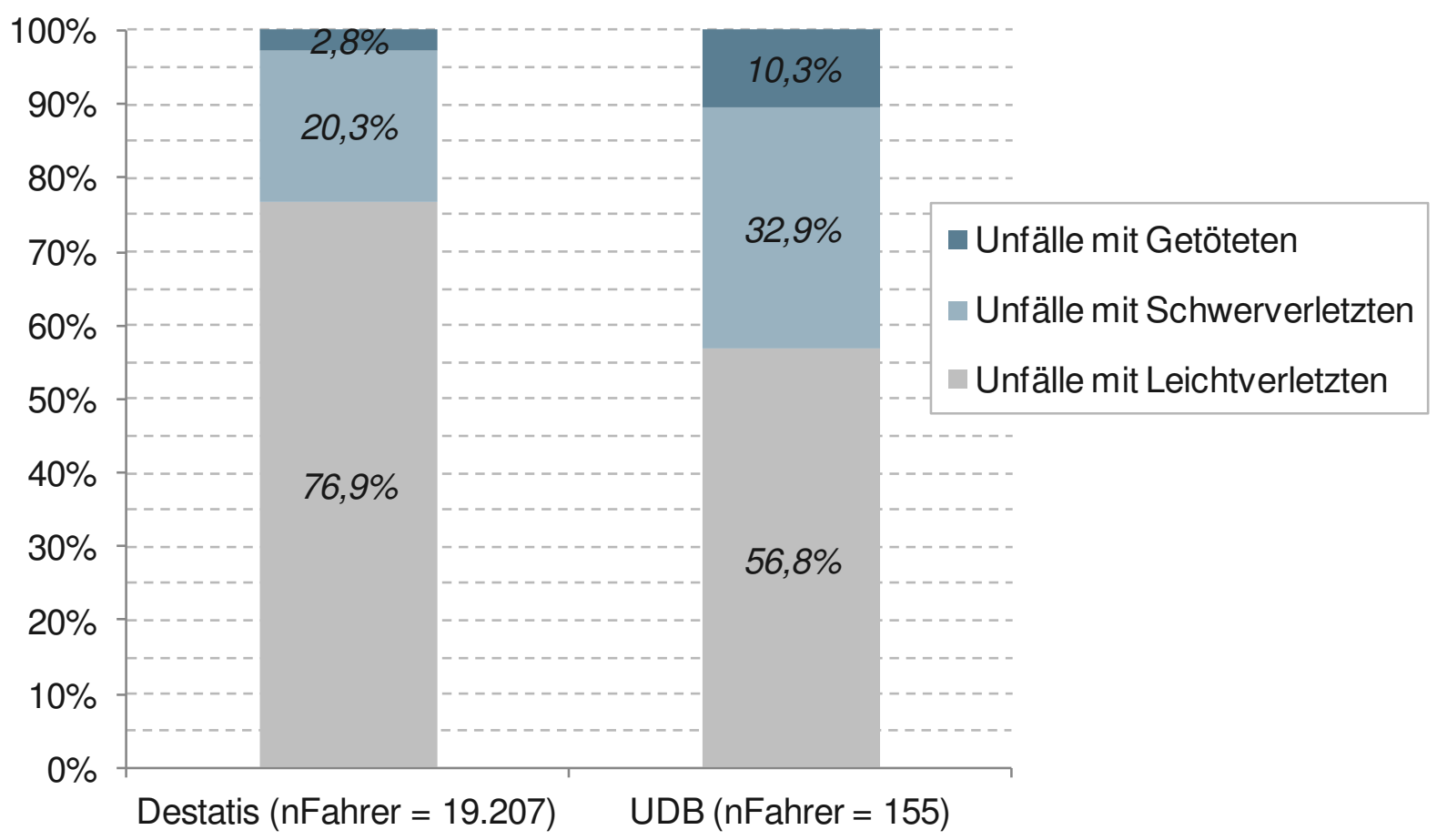

An Unfällen mit Personenschaden beteiligte Fahrer von N2-Fzg.

Abb. 9-5: Vergleich Destatis (2010-2014) [STA11-15] \& UDB nach Unfallfolgen

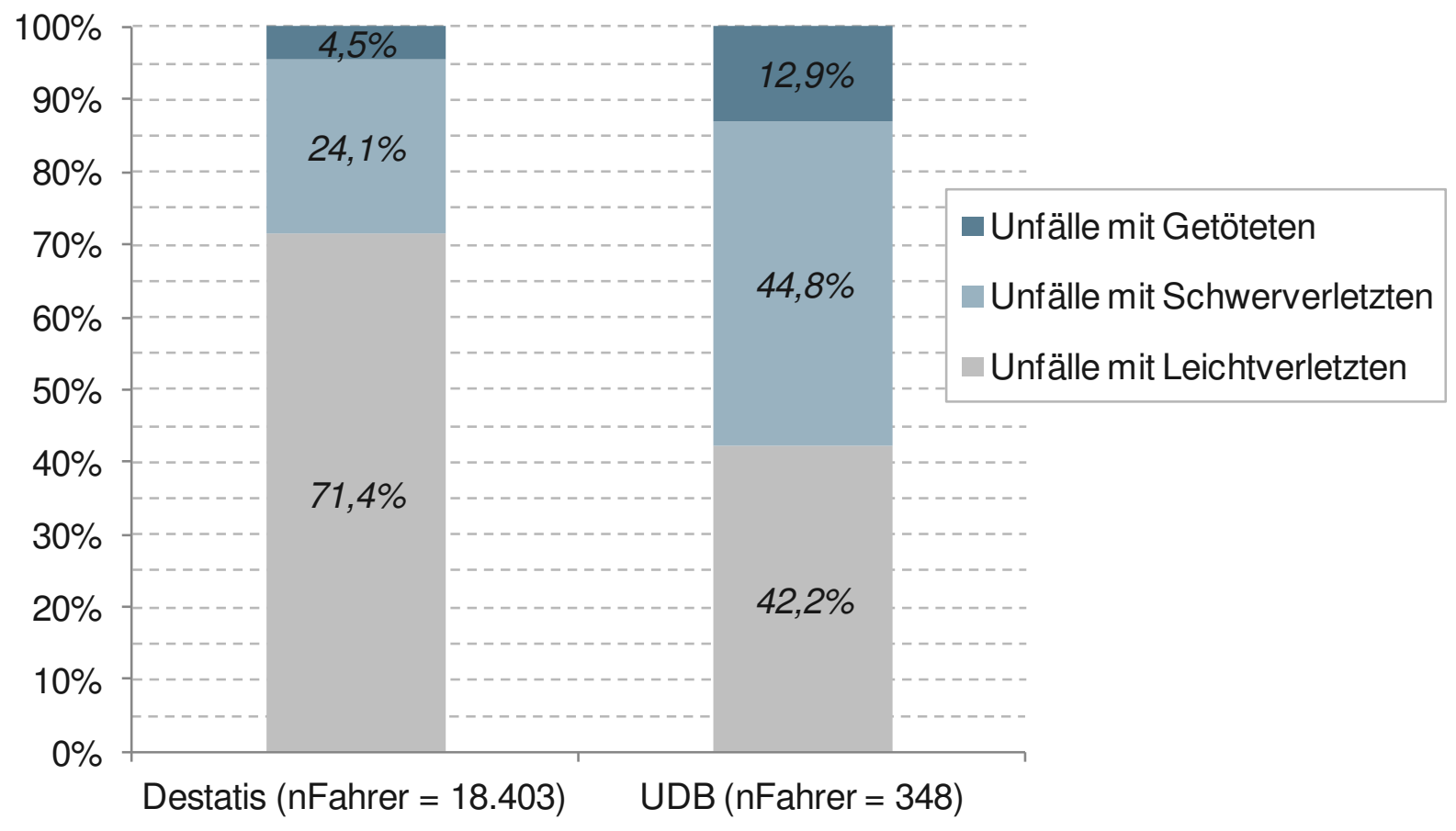

An Unfällen mit Personenschaden beteiligte Fahrer von N3-Fzg.

Abb. 9-6: Vergleich Destatis (2010-2014) [STA11-15] \& UDB nach Unfallfolgen 


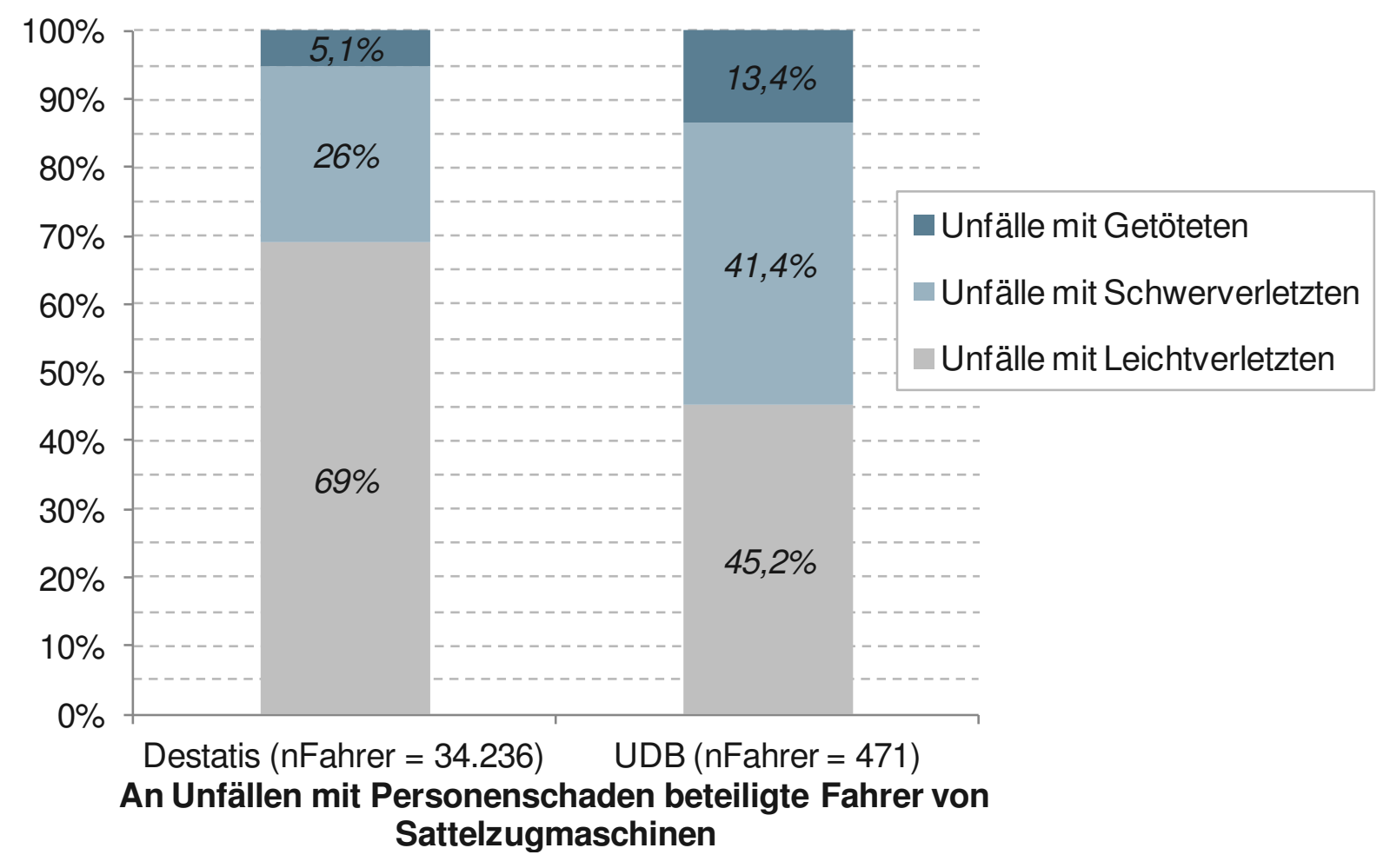

Abb. 9-7: Vergleich Destatis (2010-2014) [STA11-15] \& UDB nach Unfallfolgen 


\subsubsection{Unfallart}

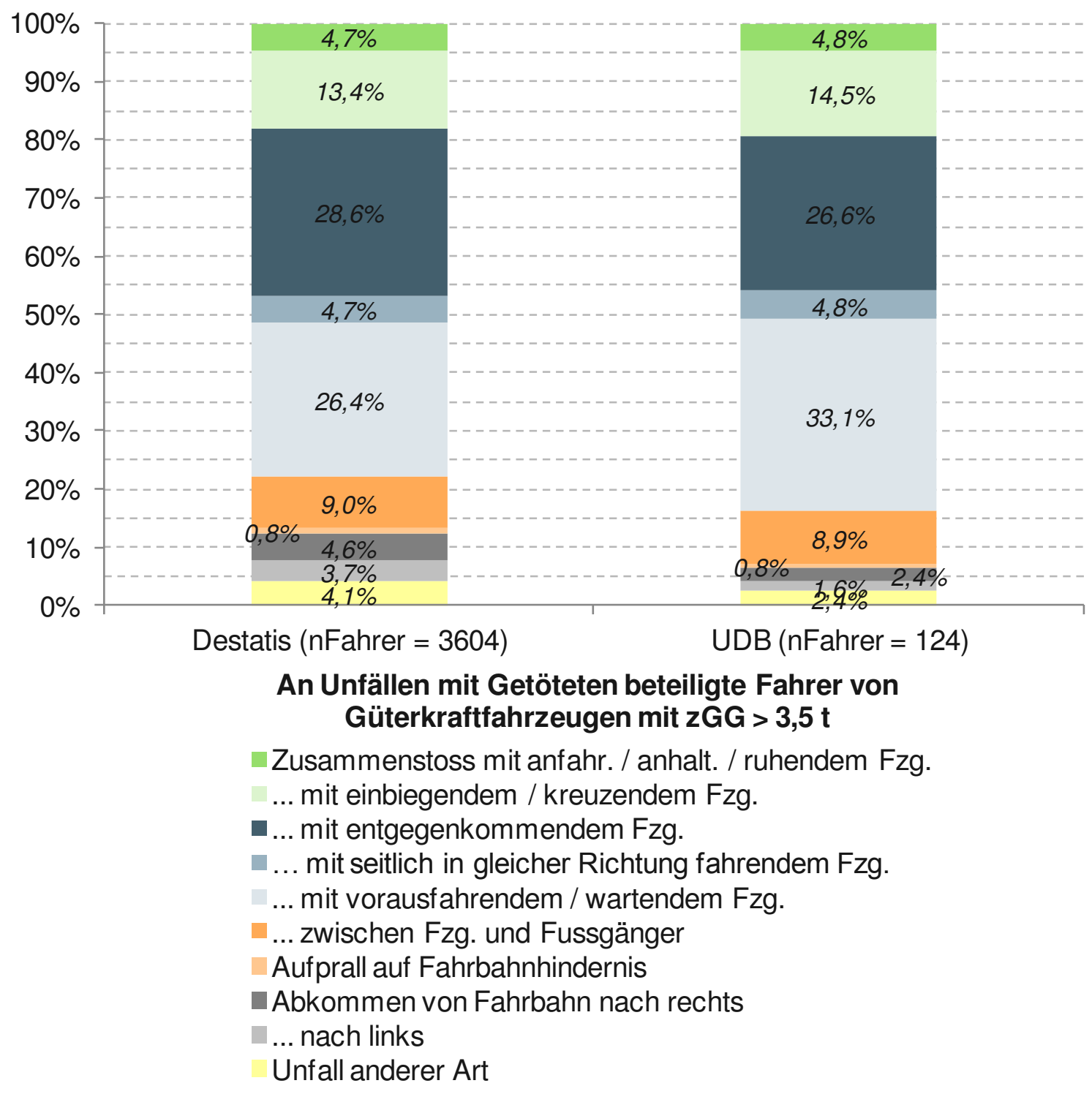

Abb. 9-8: Vergleich Destatis (2010-2014) [STA11-15] \& UDB nach Unfallart 


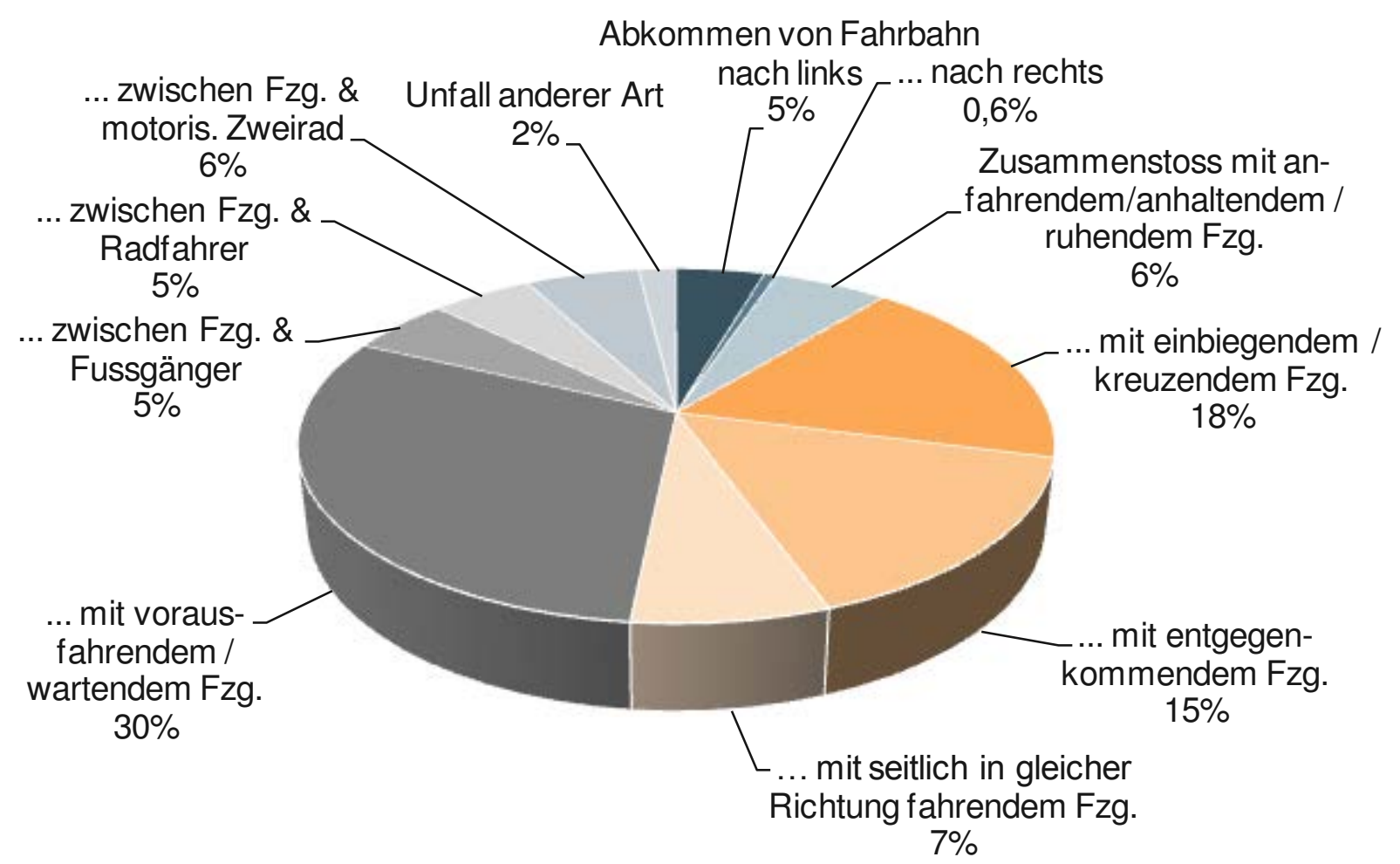

Abb. 9-9: An Unfällen beteiligte Fahrer von N2-Fahrzeugen (ohne Sattelzugmaschinen) mit zGG größer 3,5 t nach Unfallart (UDB, $n=155$ )

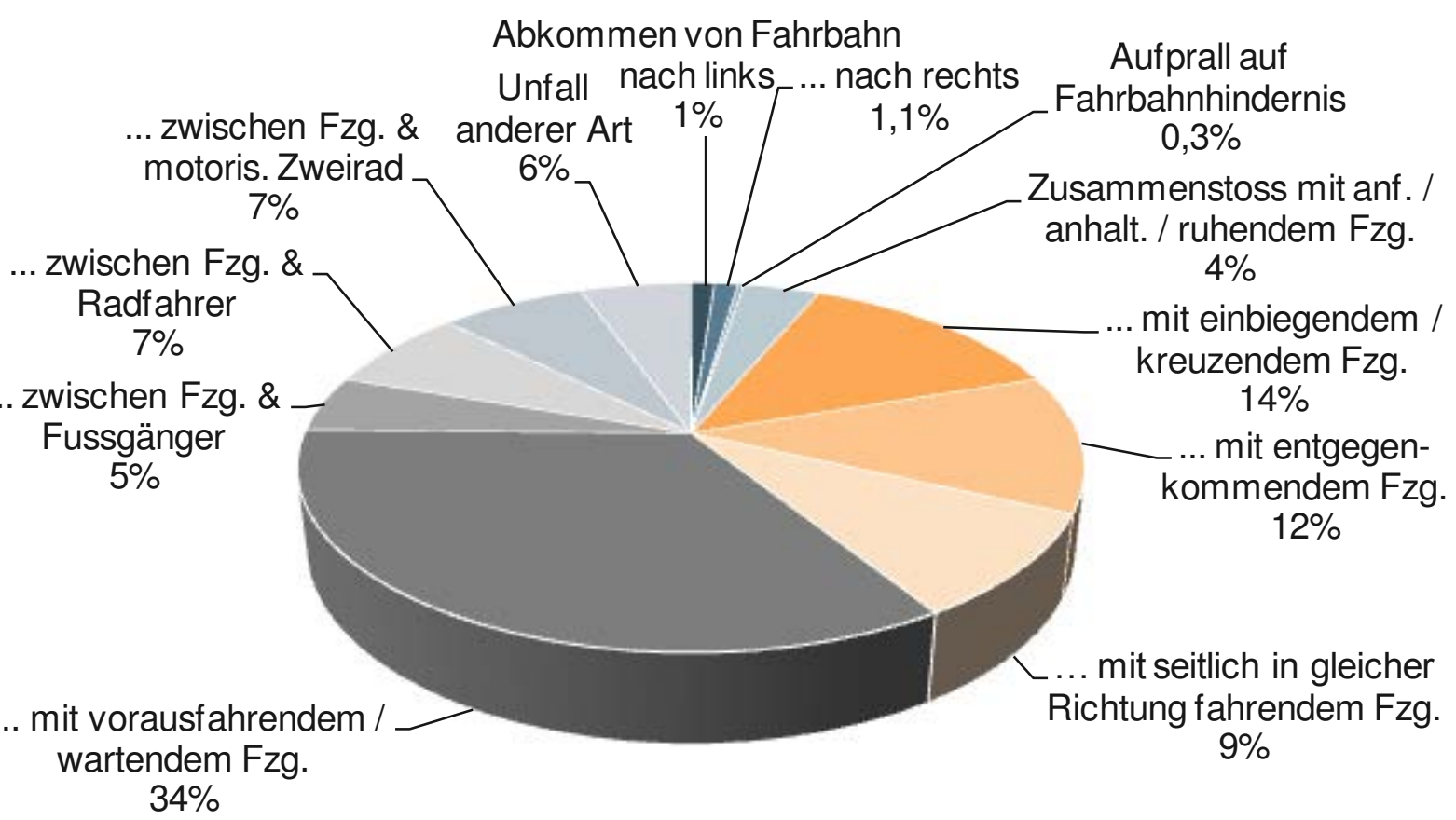

Abb. 9-10: An Unfällen beteiligte Fahrer von N3-Fahrzeugen (ohne Sattelzugmaschinen) mit zGG größer 3,5 t nach Unfallart (UDB, $n=348$ ) 


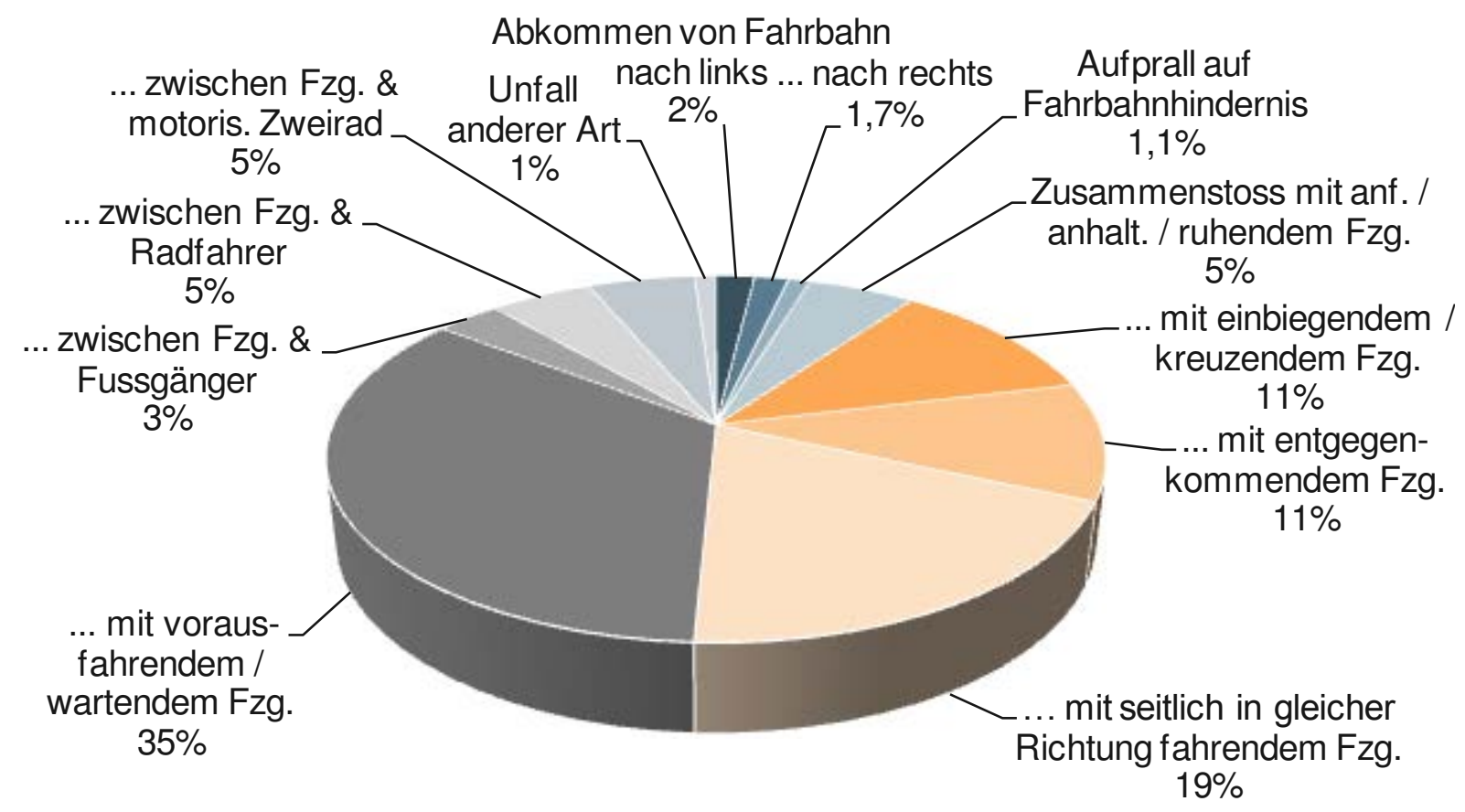

Abb. 9-11: An Unfällen beteiligte Fahrer von Sattelzugmaschinen nach Unfallart (UDB, $\mathrm{n}=471$ )

\subsubsection{Unfalltyp}

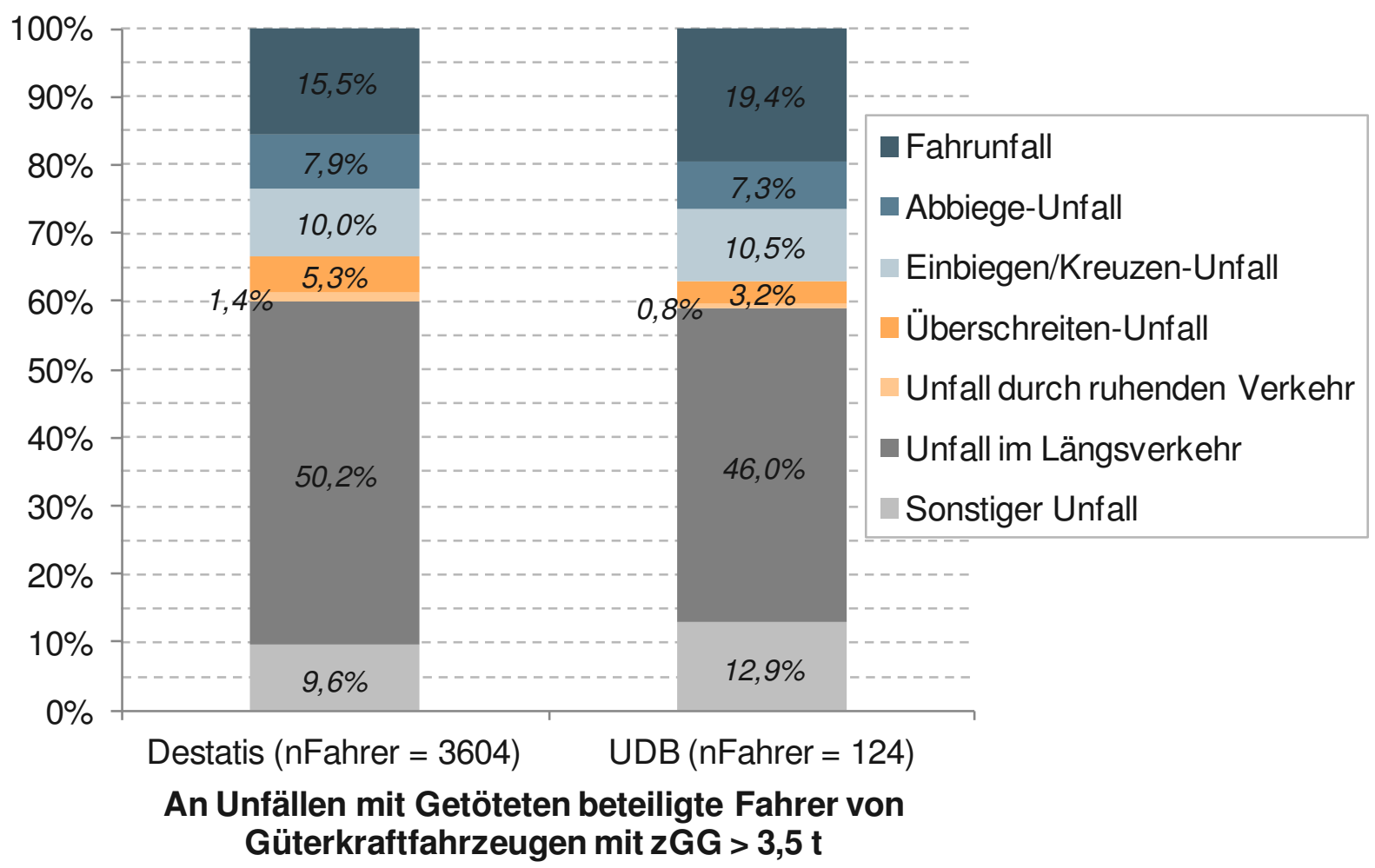

Abb. 9-12: Vergleich Destatis (2010-2014) [STA11-15] \& UDB nach Unfalltyp 


\subsubsection{Unfallgegner}

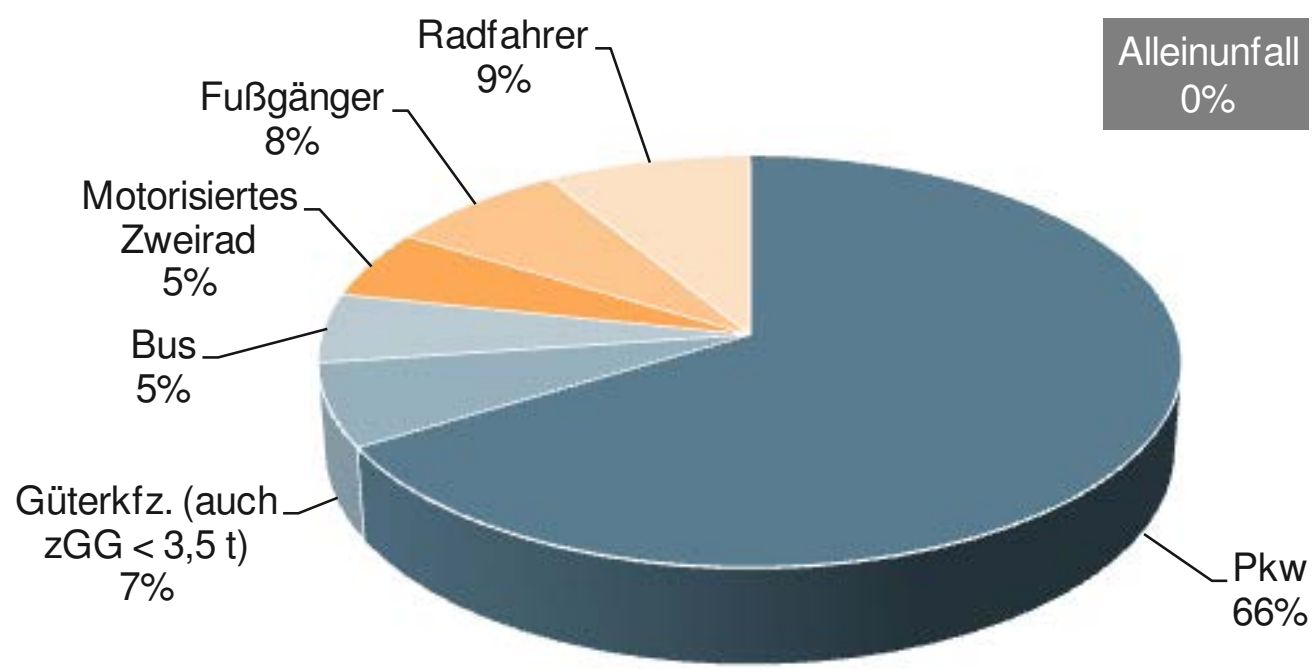

Abb. 9-13: Alleinunfälle \& Unfälle mit zwei Beteiligten von N2-Fahrzeugen (ohne Sattelzugmaschinen) nach Unfallgegnern (UDB, $\mathrm{n}=92$ )

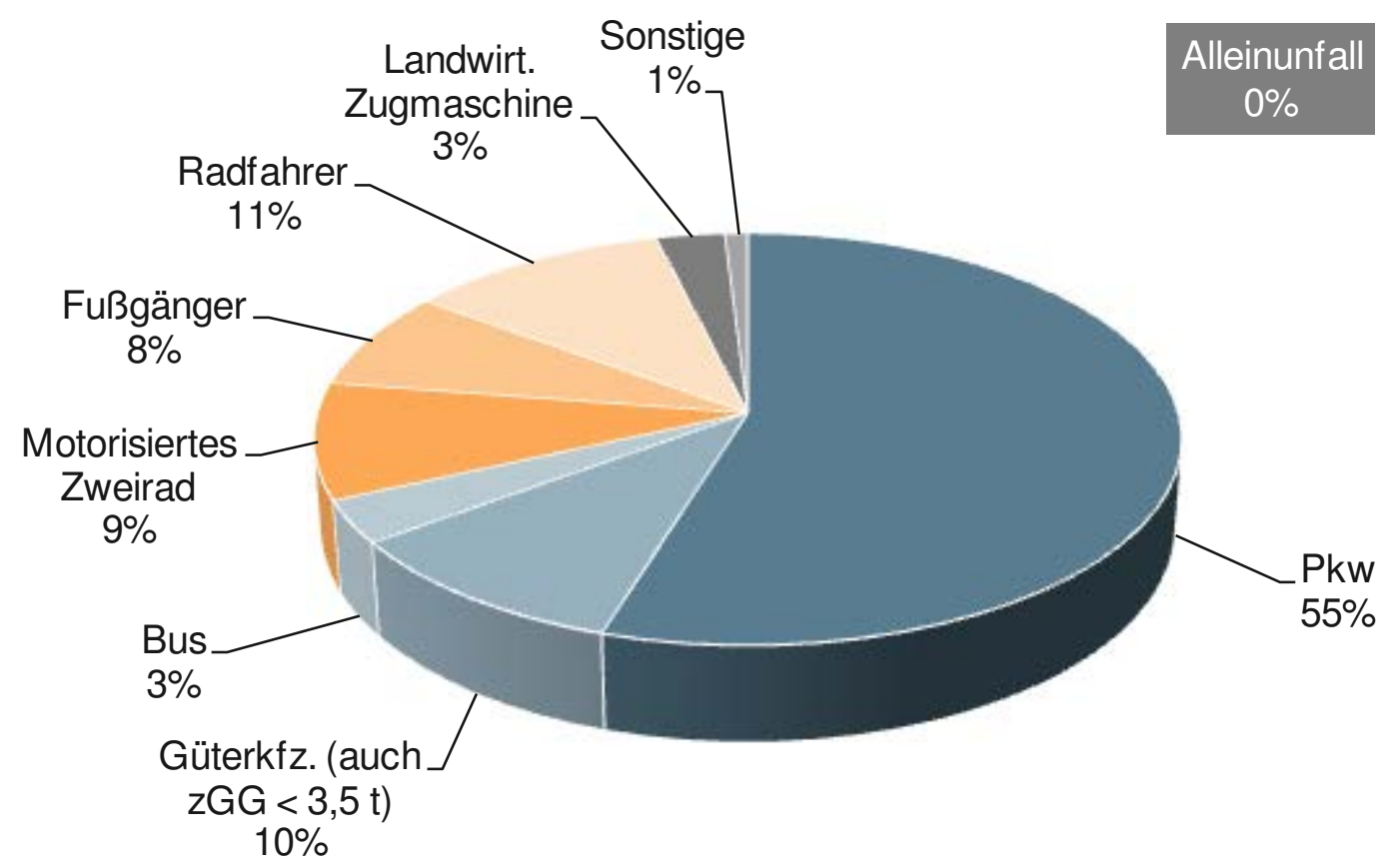

Abb. 9-14: Alleinunfälle \& Unfälle mit zwei Beteiligten von N3-Fahrzeugen (ohne Sattelzugmaschinen) nach Unfallgegnern (UDB, $\mathrm{n}=208$ ) 


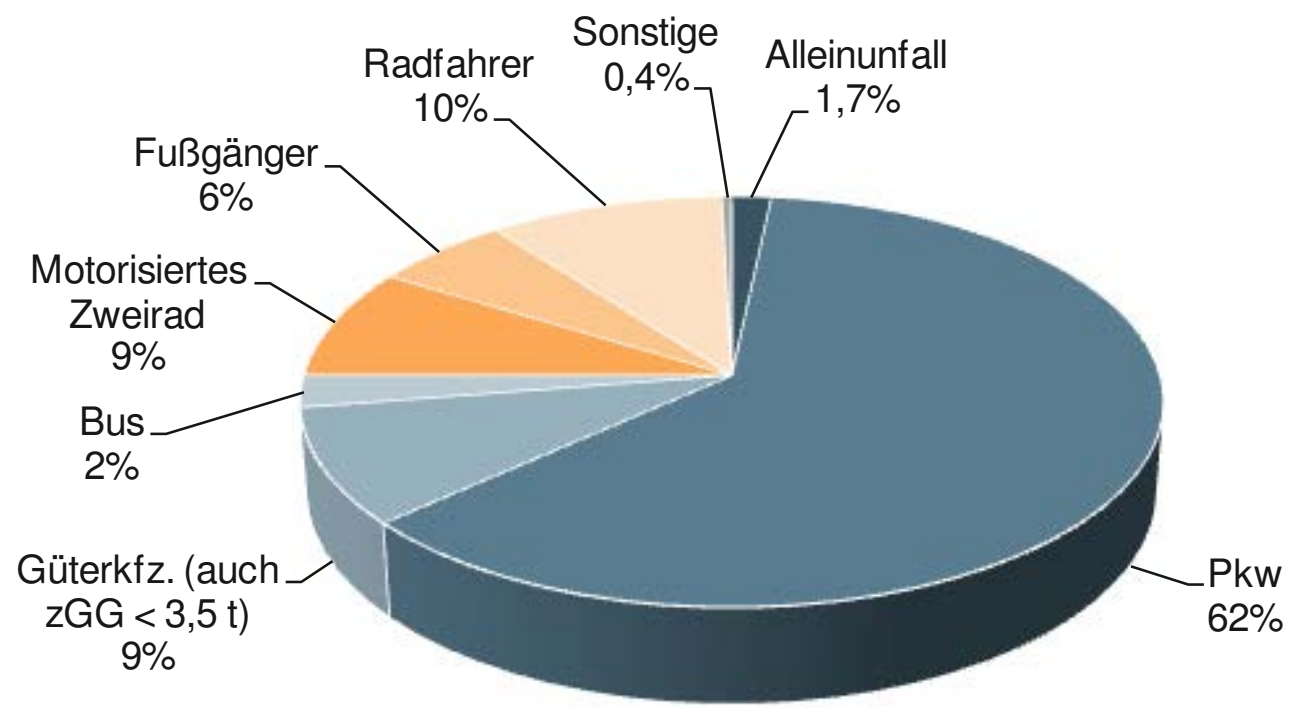

Abb. 9-15: Alleinunfälle \& Unfälle mit zwei Beteiligten von Sattelzugmaschinen nach Unfallgegnern (UDB, $n=240)$

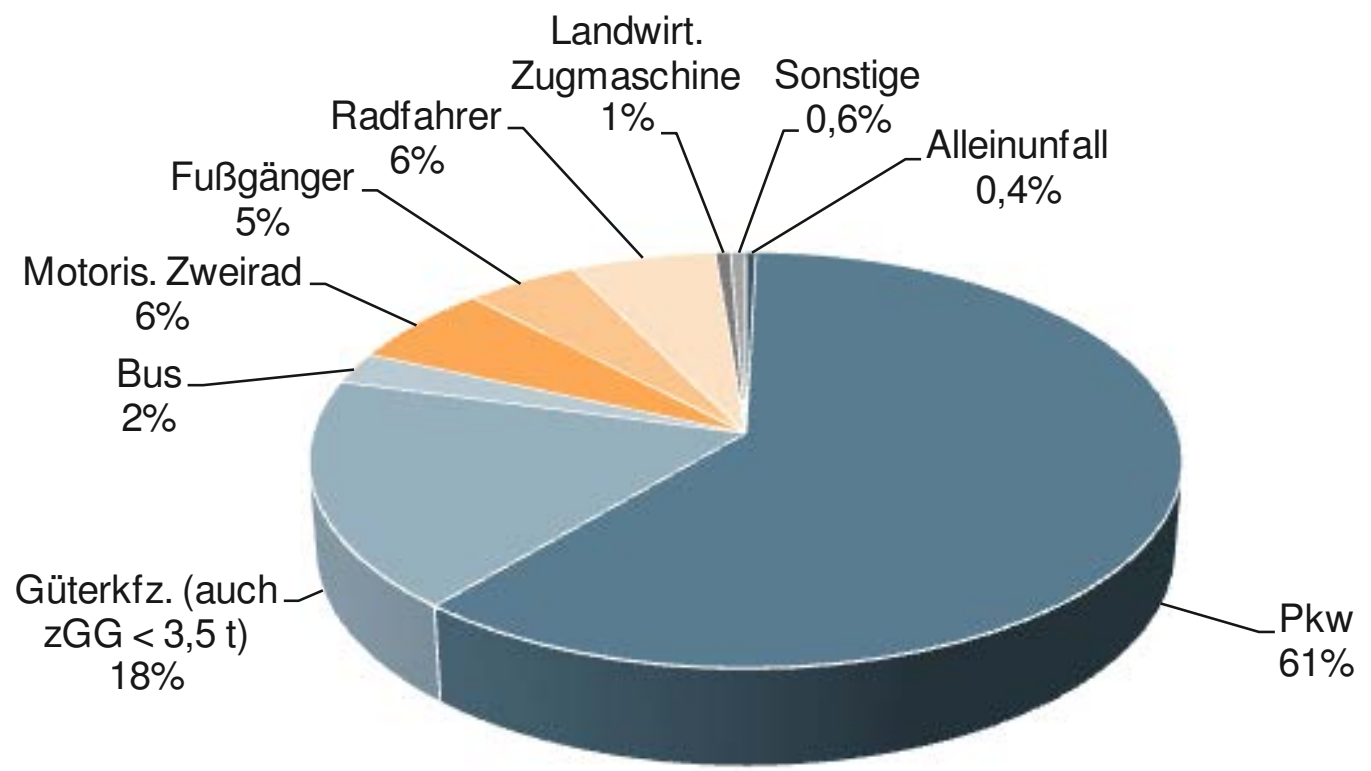

Abb. 9-16: Alleinunfälle \& Unfälle mit zwei oder mehr Beteiligten von Güterkraftfahrzeugen mit zGG größer 3,5 t nach Unfallgegnern (UDB, $\mathrm{n}=834$, $\mathrm{n}_{\text {Beteiligte }}=941$ ) 
9.2 Weitere Auswertungen der Unfalldatenbank der Versicherer

9.2.1 Fahreralter

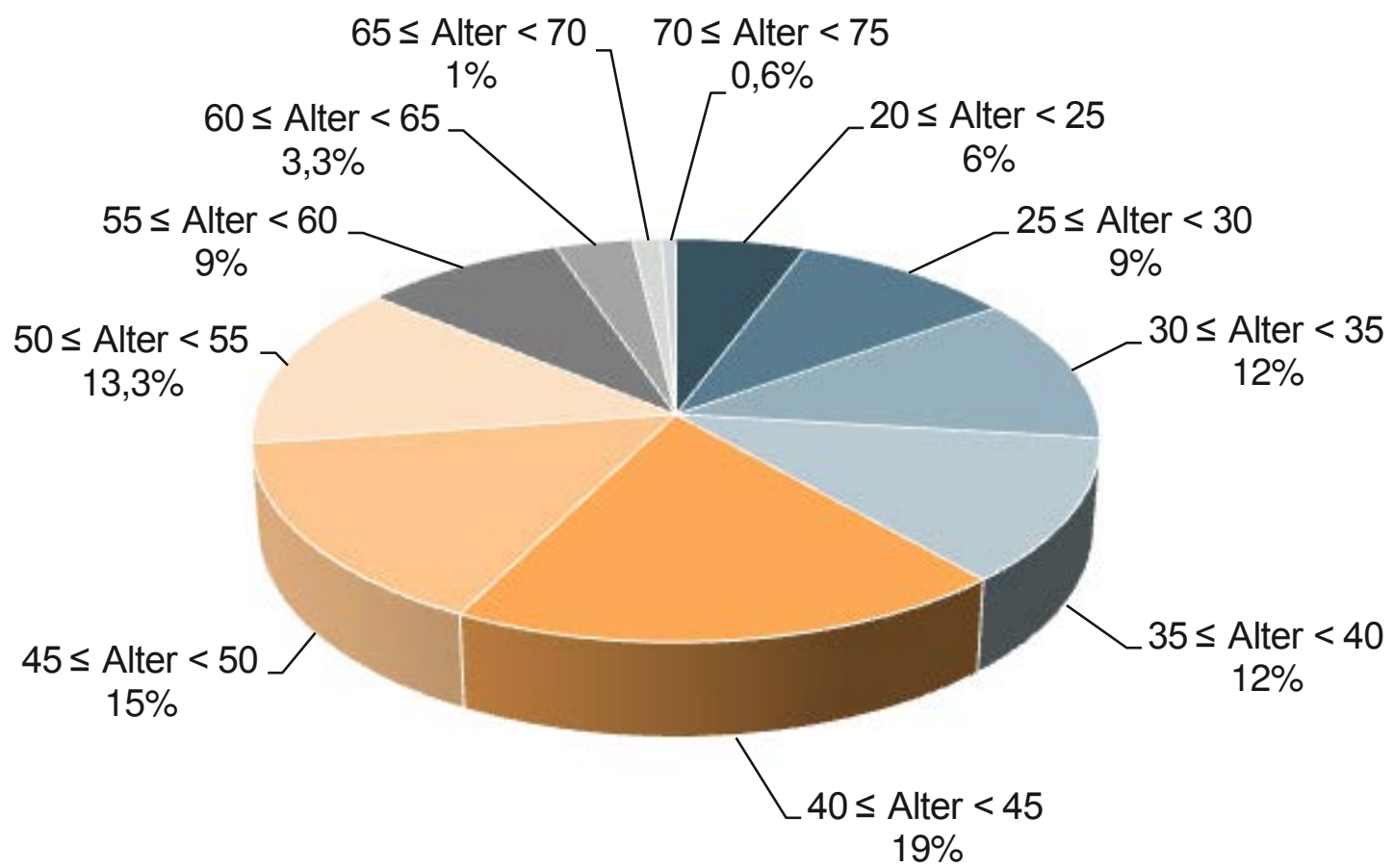

Abb. 9-17: Als Unfallhauptverursacher (Beteiligter 01) eingestufte Fahrer von Güterkraftfahrzeugen mit zGG größer 3,5 t nach Fahreralter (UDB, $n=480$ )

\subsubsection{Kollisionsart}

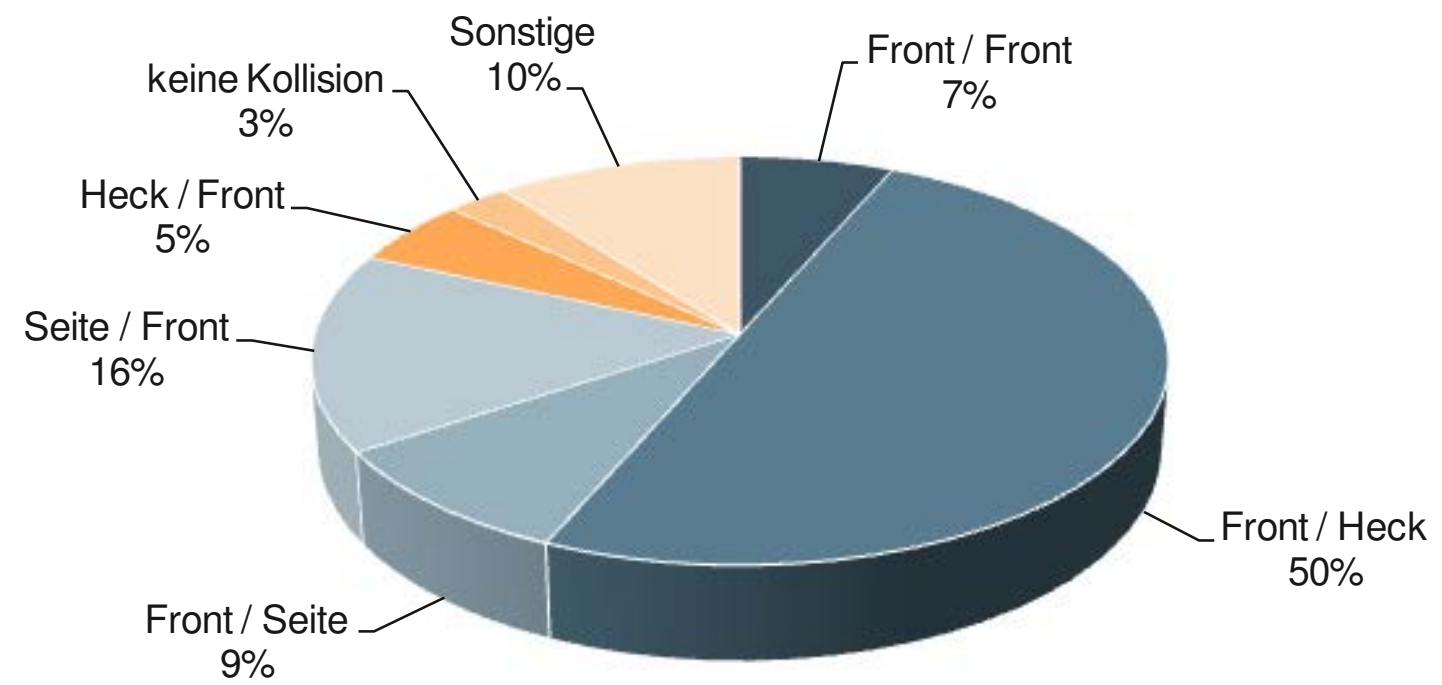

Abb. 9-18: Anteil der Kollisionsarten mit Beteiligung von N2-Fahrzeugen (ohne Sattelzugmaschinen), N2-Fahrzeug-Fahrer Hauptverursacher (Beteiligter 01) (UDB, n=76) 


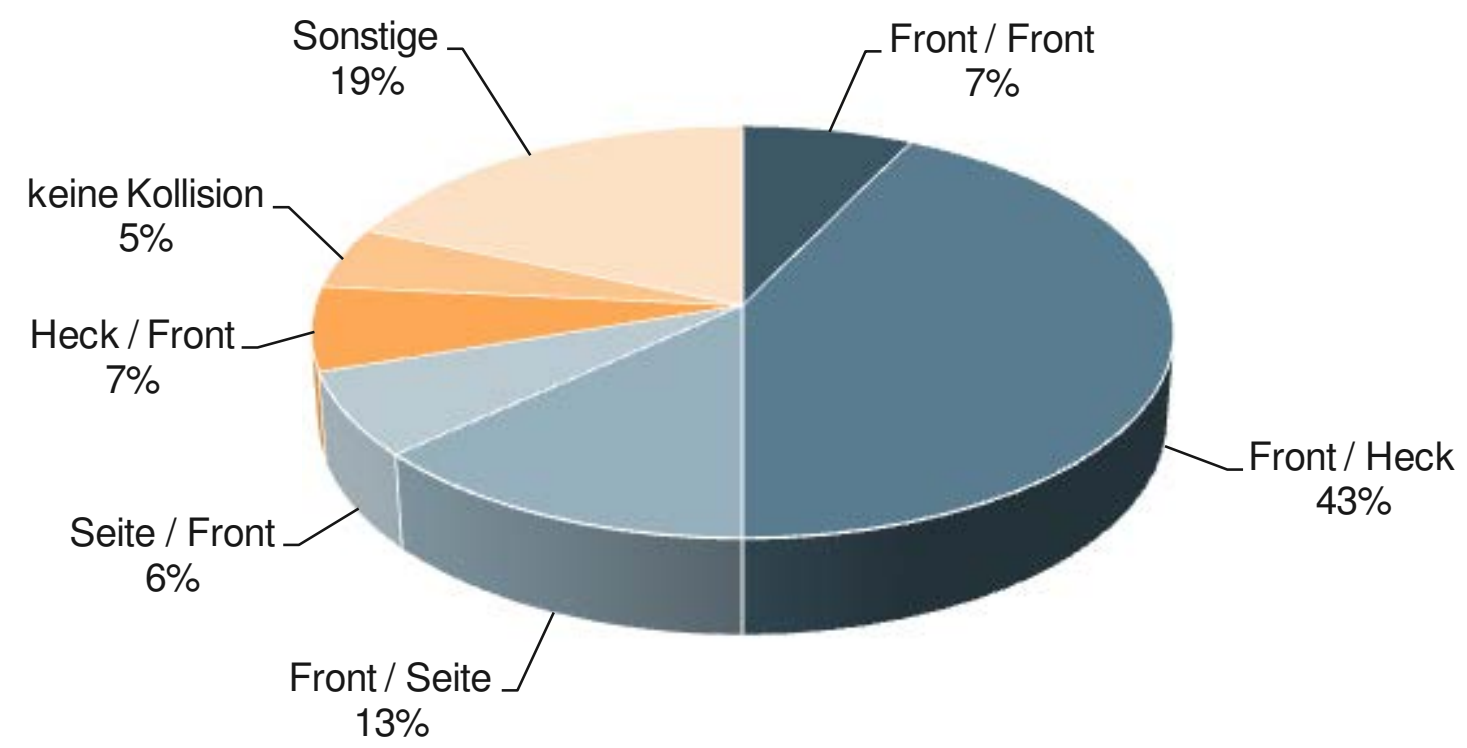

Abb. 9-19: Anteil der Kollisionsarten mit Beteiligung von N3-Fahrzeugen (ohne Sattelzugmaschinen), N3-Fahrzeug-Fahrer Hauptverursacher (Beteiligter 01) (UDB, n=276)

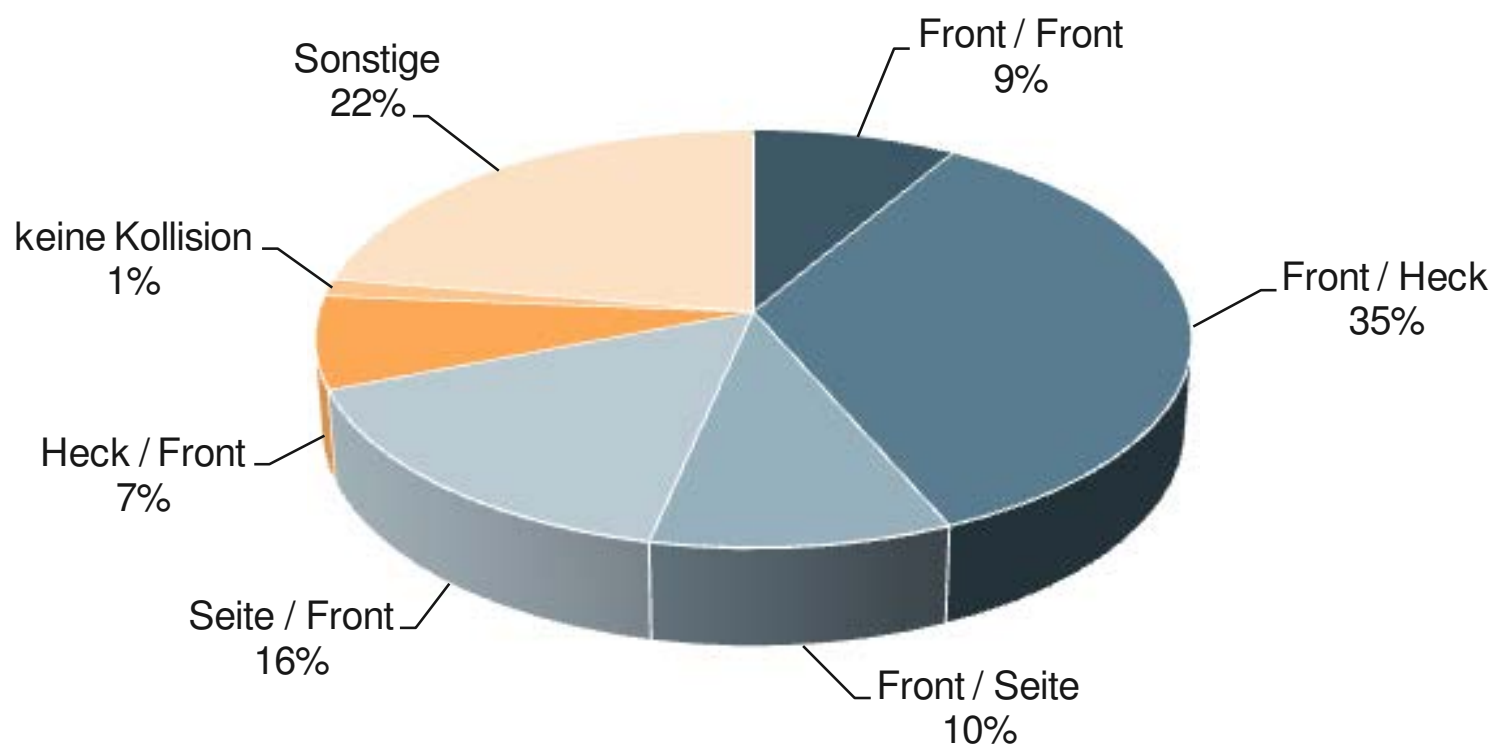

Abb. 9-20: Anteil der Kollisionsarten mit Beteiligung von Sattelzugmaschinen, Sattelzugmaschinen-Fahrer Hauptverursacher (Beteiligter 01) (UDB, $n=393$ ) 


\subsubsection{Hauptanstoß}

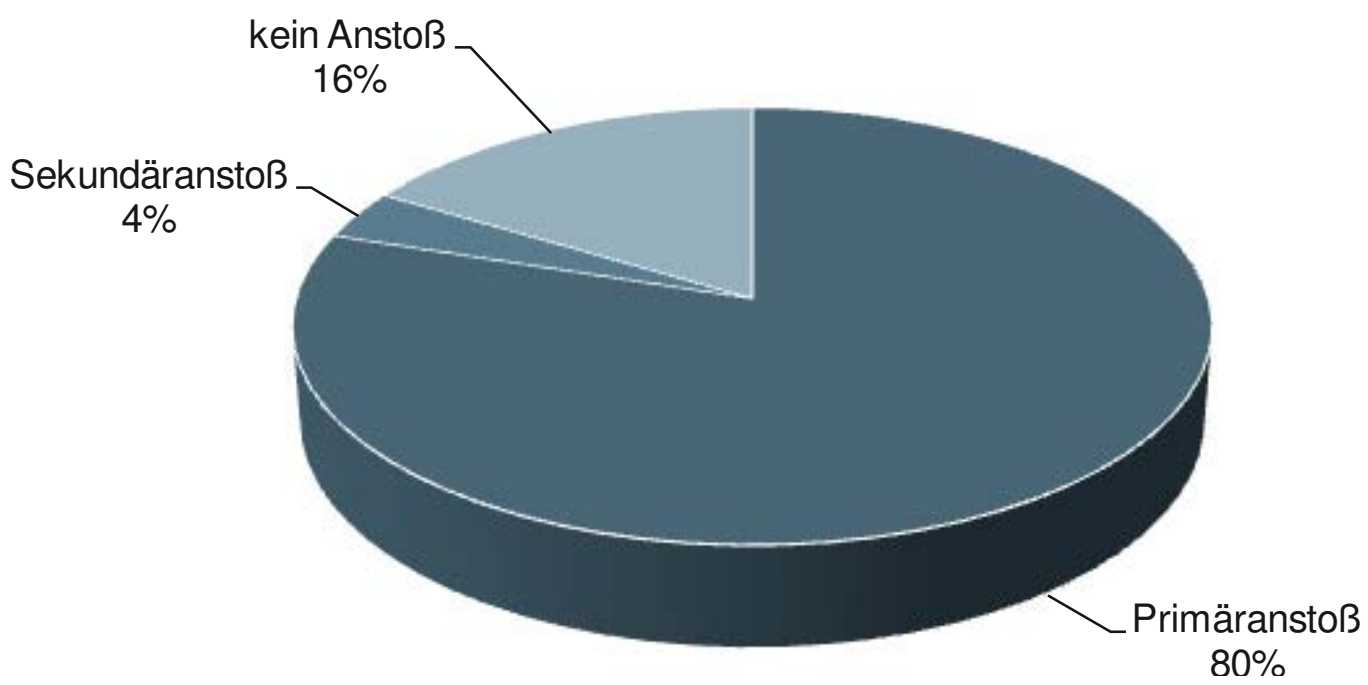

Abb. 9-21: Hauptanstoß für Güterkraftfahrzeuge mit zGG größer 3,5 t, deren Fahrer Hauptverursacher (Beteiligter 01) ist (UDB, $n=577$ )

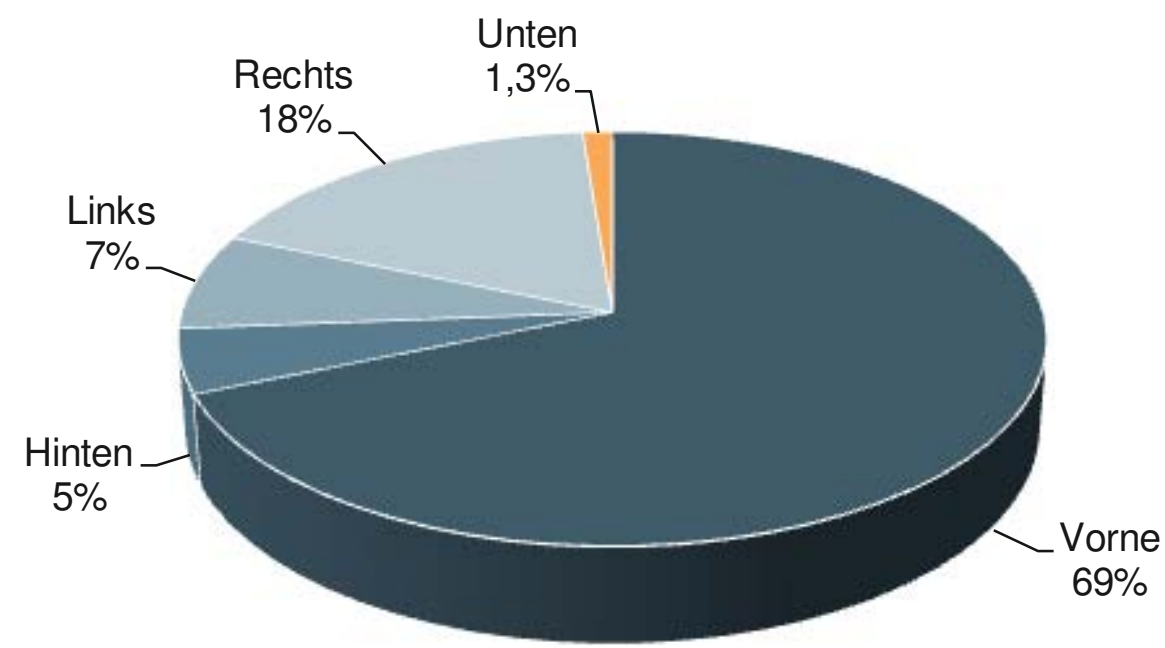

Abb. 9-22: Seite des Hauptanstoßes bei N2-Fahrzeugen, deren Fahrer Hauptverursacher (Beteiligter 01) ist $(n=80)$ 


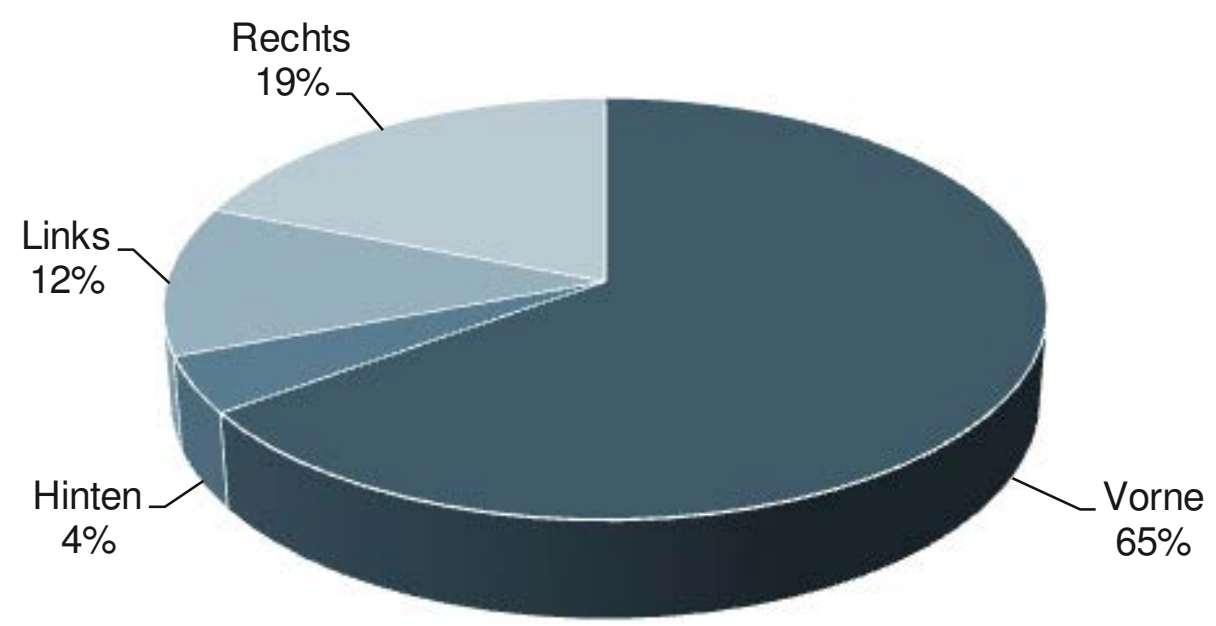

Abb. 9-23: Seite des Hauptanstoßes bei N3-Fahrzeugen, deren Fahrer Hauptverursacher (Beteiligter 01) ist $(n=183)$

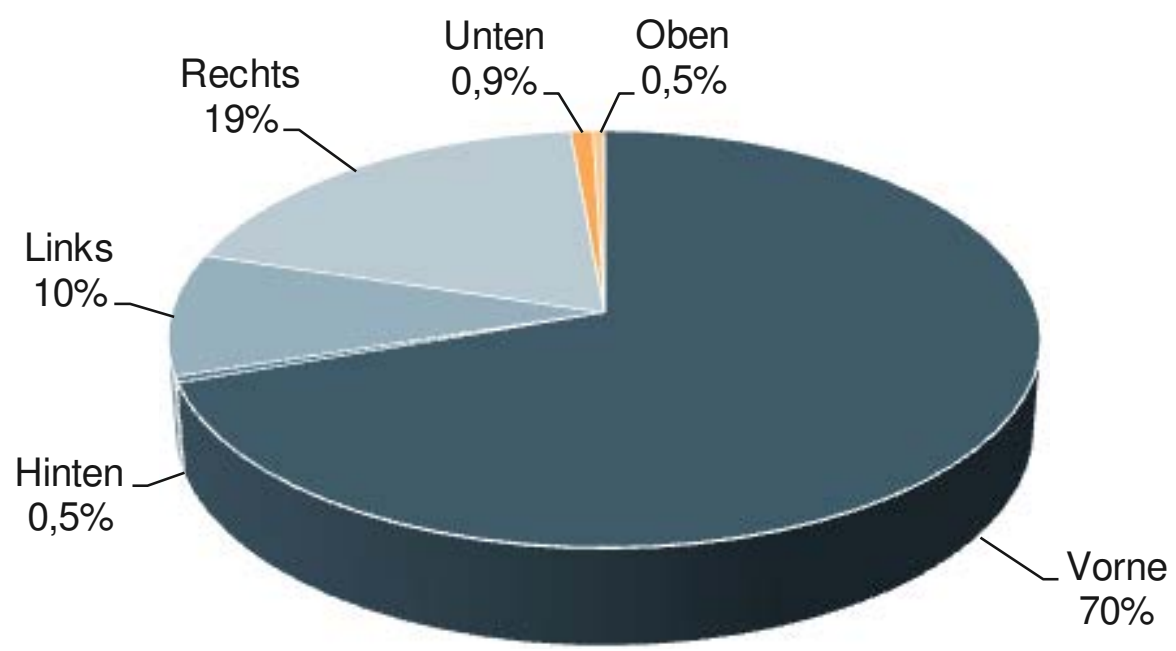

Abb. 9-24: Seite des Hauptanstoßes bei Sattelzugmaschinen, deren Fahrer Hauptverursacher (Beteiligter 01) ist $(n=183)$ 


\subsection{4 Überdeckungsgrad}

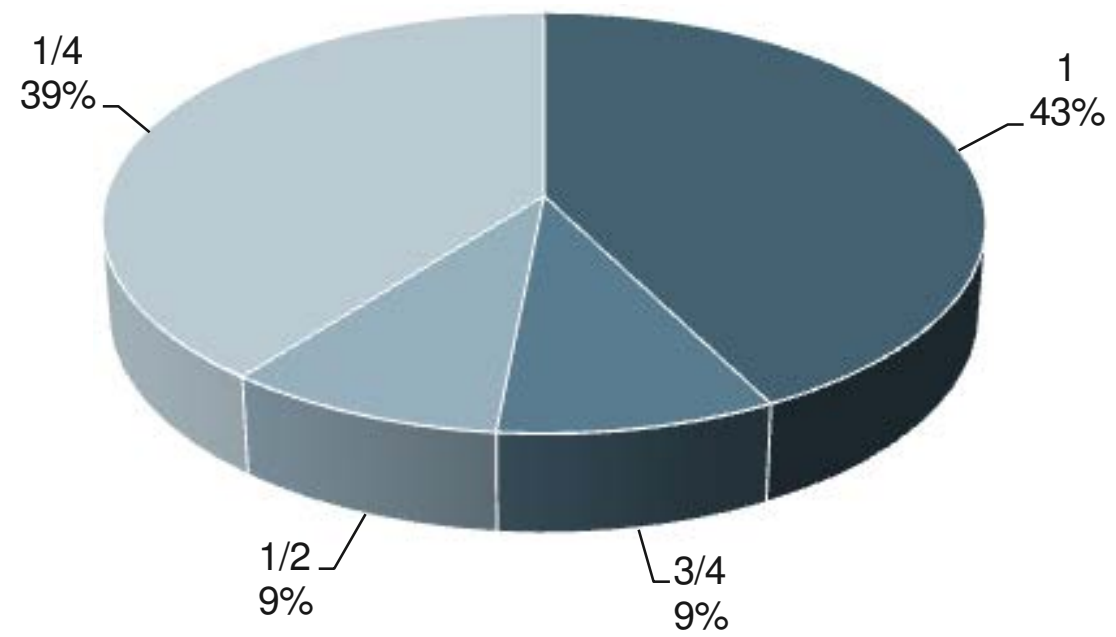

Abb. 9-25: Überdeckungsgrad beim Frontalanprall bei N2-Fahrzeugen, deren Fahrer Hauptverursacher (Beteiligter 01) ist $(n=33)$

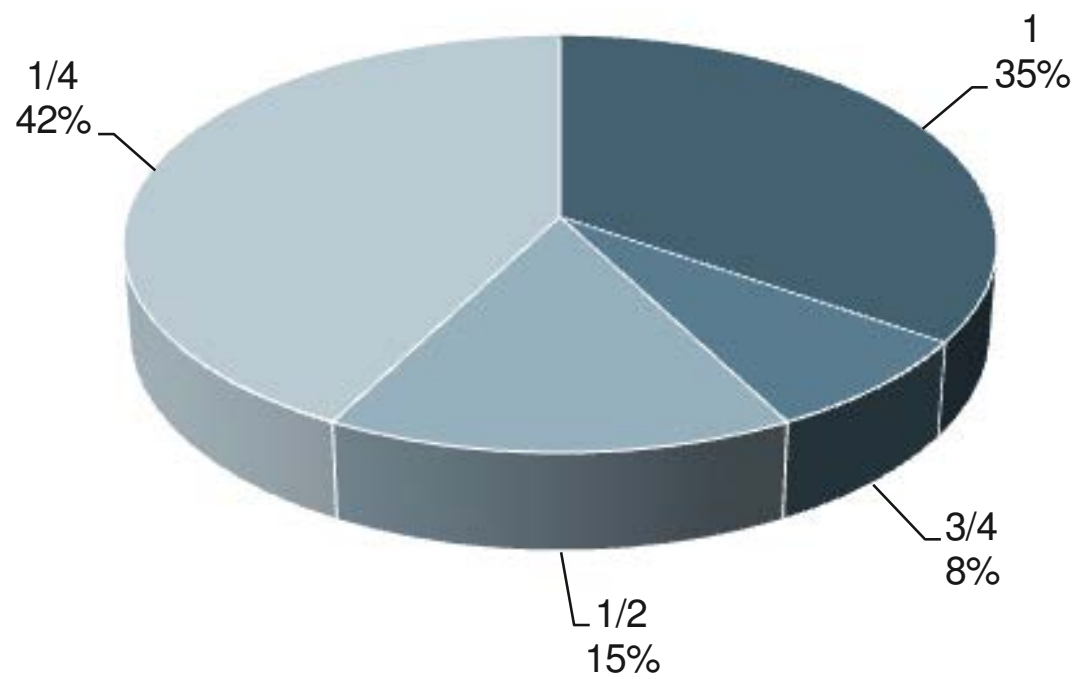

Abb 9-26: Überdeckungsgrad beim Frontalanprall bei N3-Fahrzeugen, deren Fahrer Hauptverursacher (Beteiligter 01) ist $(n=78)$ 


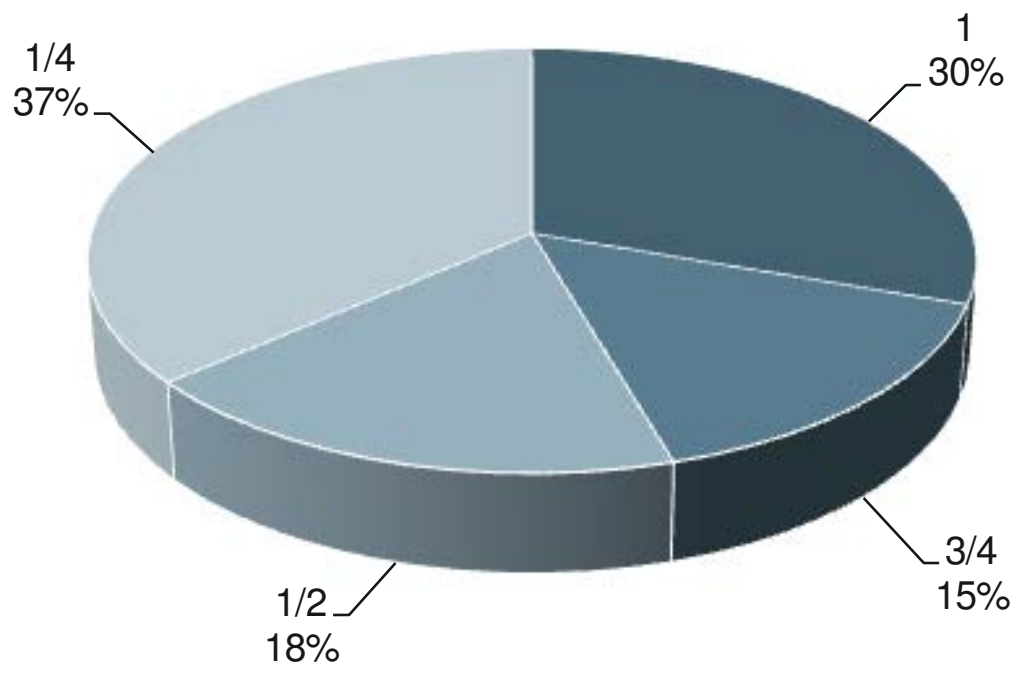

Abb. 9-27: Überdeckungsgrad beim Frontalanprall bei Sattelzugmaschinen, deren Fahrer Hauptverursacher (Beteiligter 01) ist $(n=99)$ 


\subsection{Seitenwindmodell - Mechanik des Kippvorgangs}

Trotz konstanter Windkraft nimmt das resultierende Kippmoment im Zuge des ortsfesten Kraftangriffspunktes bis zu einem hohen Wankwinkel immer stärker zu.

$\Rightarrow$ Daher setzt in der Simulation bei Radlastverlust auch stets Kippen ein.

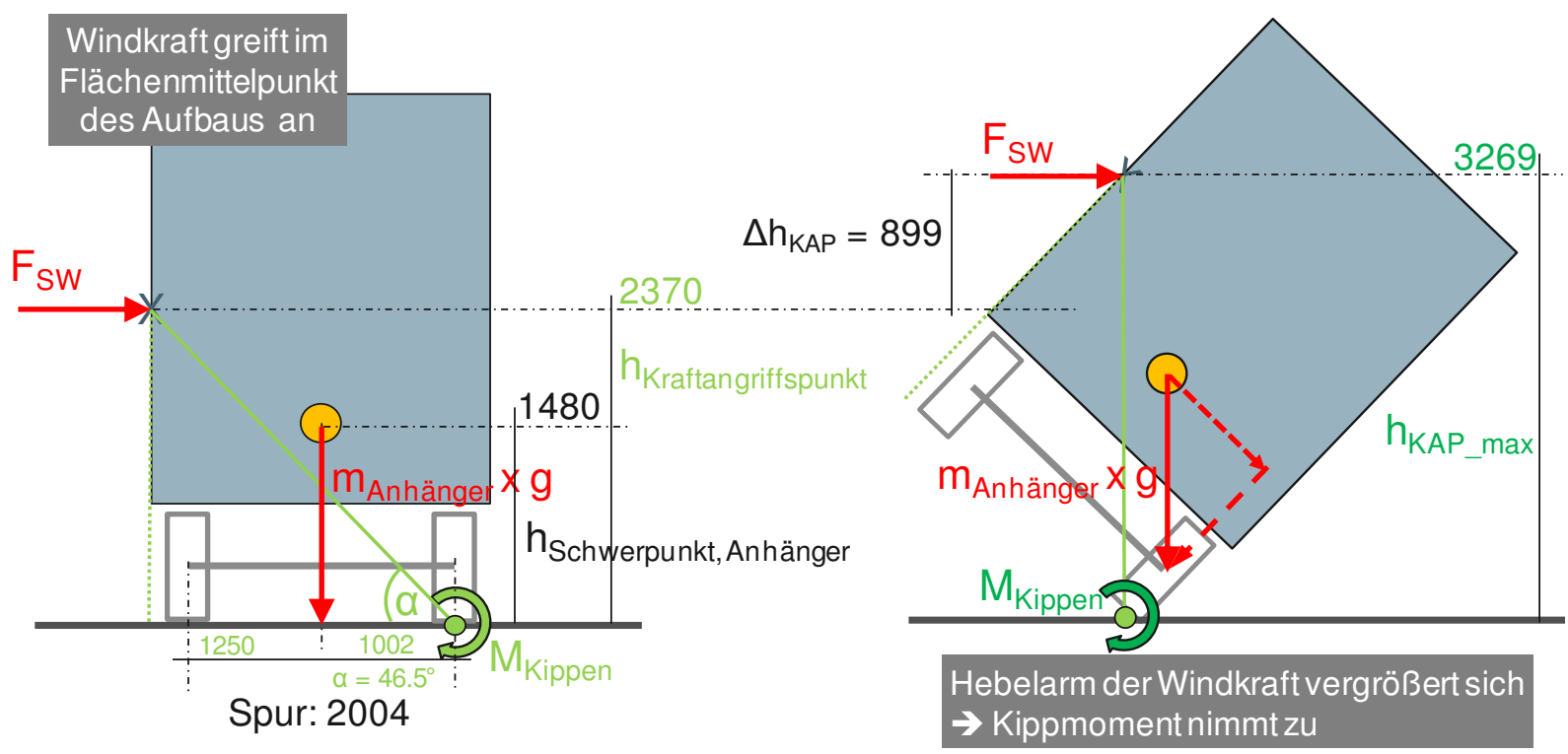

Abb. 9-28: Mechanik des Kippvorgangs beim Anhänger des 12 t-Gliederzugs 

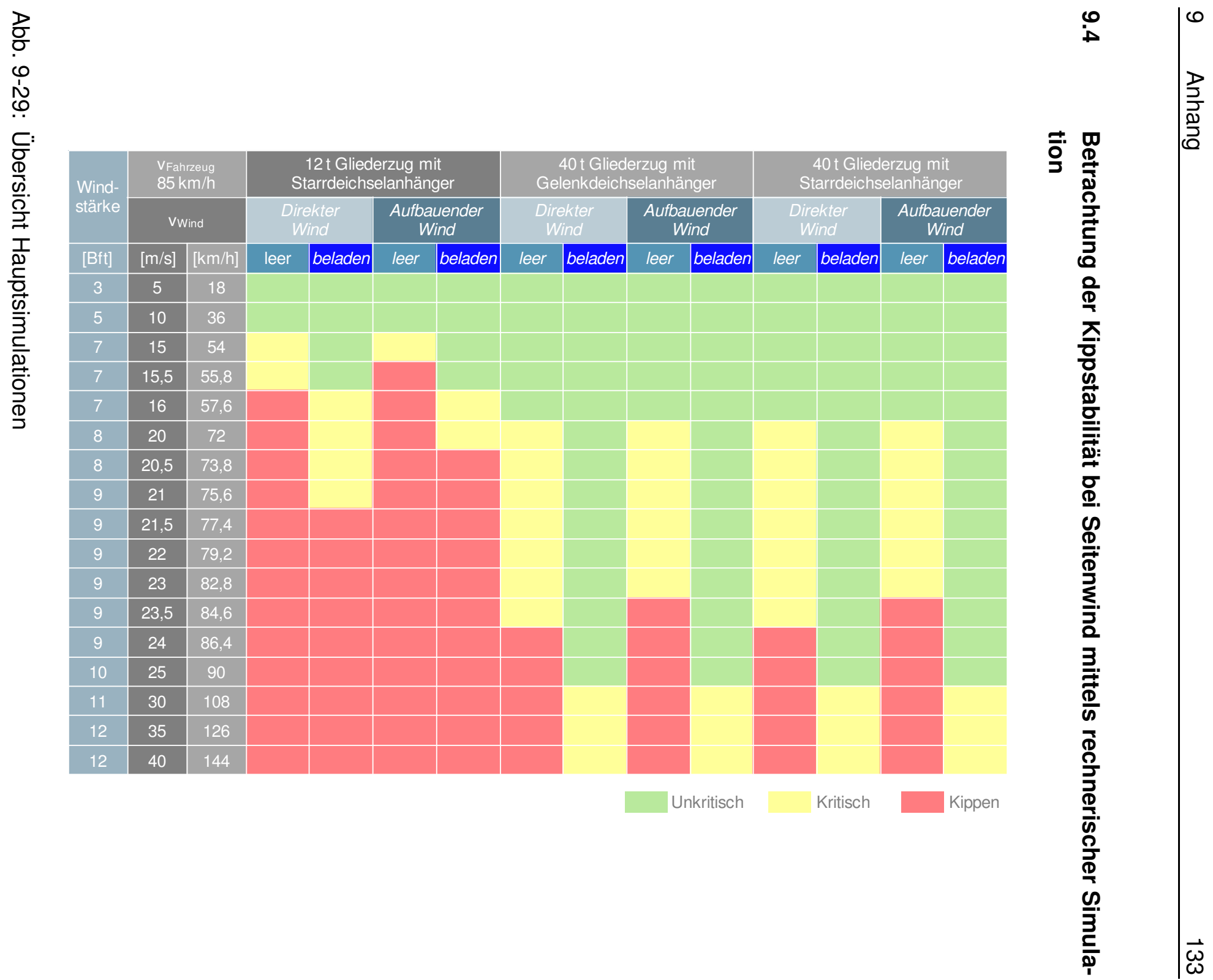


\section{GDV}

DIE DEUTSCHEN VERSICHERER

Gesamtverband der Deutschen Versicherungswirtschaft e. V.

Wilhelmstraße 43 / 43G, 10117 Berlin

Postfach 0802 64, 10002 Berlin

Telefon 030 / 2020 - 50 00, Fax 030 / 2020 - 6000

Internet: www.gdv.de, www.udv.de 